\title{
Ionization and plasma dynamics of single large xenon clusters in superintense XUV pulses
}

\author{
vorgelegt von \\ Diplom-Physikerin \\ Daniela Rupp \\ aus Augsburg \\ von der Fakultät II - Mathematik und Naturwissenschaften \\ der Technischen Universität Berlin \\ zur Erlangung des akademischen Grades \\ Doktor der Naturwissenschaften \\ - Dr. rer. nat. - \\ genehmigte Dissertation
}

Promotionsausschuss:

Vorsitzender:

Prof. Dr. Mario Dähne

Berichter/Gutachter: Prof. Dr. Thomas Möller

Berichter/Gutachter: Prof. Dr. Karl-Heinz Meiwes-Broer

Tag der wissenschaftlichen Aussprache: 30.05.2013

Berlin 2013

D 83 


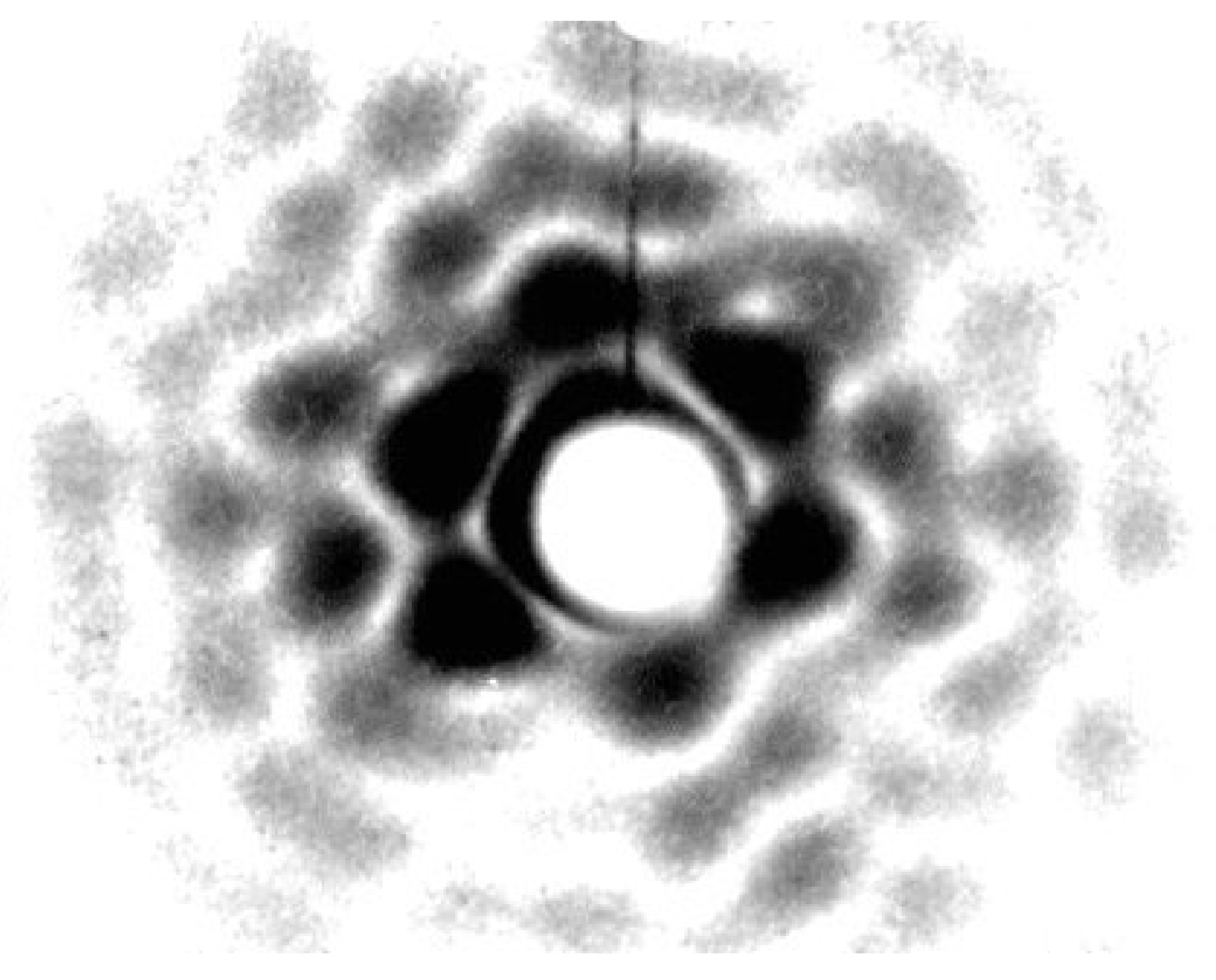

It is structure that we look for whenever we try to understand anything. All science is built upon this search; Linus Pauling in [1] 


\section{Abstract}

In this work fundamental mechanisms of the interaction between matter and strong shortwavelength pulses have been investigated. For the experiments ultrashort and highly intense light pulses in the extreme-ultraviolet spectral regime from the Free-Electron Laser in Hamburg FLASH have been used. They provide access to new fields of experiments such as imaging of single nanometer-sized structures.

Rare gas clusters in the gas phase were investigated as ideal model systems for light-matter interaction. From the scattering patterns of individual clusters, their size and shape as well as the power density they were exposed to can be determined. But also light-induced changes in the clusters on a femtosecond time scale are encoded in the scattered light. By combining single cluster imaging with the coincident measurement of ion spectra, it is possible to sort the single shot data based on the information in the scattering patterns. Thus, the averaging of cluster size distributions and FEL power density profiles which has been apparent in virtually all previous studies on rare gas clusters can be overcome. The well-defined conditions in the single shot data sorted for cluster size yield unique insight into the nanoplasma dynamics.

Large xenon clusters with diameters of hundreds of nanometers up to microns could be produced and investigated for the first time. Their scattering patterns reveal a complex, hailstone-like structure, yielding new insight into cluster morphology and growth mechanisms.

The characteristic energy distributions of the ions determined from the time-of-flight spectra of very large single xenon clusters indicate that only ions from the outermost atomic monolayer of the cluster explode off. At the same time, the highly efficient recombination in the remaining, quasi-neutral nanoplasma allows for most atoms in the cluster to return back to neutral state.

From the corresponding scattering patterns intensity profiles can be extracted. Mie theory yields insight into the optical properties of the clusters. In the intensity profiles of large clusters, modulations are observed which indicate the development of a core-shell system within the nanoplasma. The core and a shell that is up to $50 \mathrm{~nm}$ thick differ in the optical properties, yielding insight into ultrafast rearrangements of the electronic structure of the cluster. 


\section{Kurzfassung}

In dieser Arbeit wurden fundamentale Mechanismen der Wechselwirkung von Materie mit intensiven, kurzwelligen Laserpulsen untersucht. Für die Experimente wurden ultrakurze, hochintensive Lichtpulse im extrem-ultravioletten Spektralbereich verwendet, die am FreieElektronen-Laser in Hamburg FLASH seit einigen Jahren zur Verfügung stehen. Sie bieten Zugang zu neuen Forschungsfeldern wie der Strukturuntersuchung einzelner Nano-Objekte durch direkte Abbildung.

Edelgascluster wurden als ideale Modellsysteme für Studien der Licht-Materie-Wechselwirkung verwendet. Aus den Streubildern einzelner Cluster kann deren Form und Größe, sowie die Intensität des Lichts bestimmt werden, mit dem die Cluster getroffen wurden. Darüber hinaus beeinflussen auch lichtinduzierte Änderungen in der elektronischen Struktur der Cluster auf einer Zeitskala von Femtosekunden die Charakteristik des gestreuten Lichts. Die Abbildung einzelner Cluster wird durch die gleichzeitige Messung der zugehörigen Ionenspektren ergänzt. Hierdurch können die aufgenommenen Einzelschussdaten nach Informationen aus den Streubildern sortiert werden. So wird die Mittlung über Clustergrößenverteilung und Intensitätsprofil des Lasers vermieden, die in beinahe allen vorangegangenen Studien an Edelgasclustern miteinbezogen werden musste. Die genau definierten Bedingungen in den größenselektierten Einzelschussdaten erlauben ganz neue Einsichten in die Nanoplasmadynamiken der Cluster.

Sehr große Xenoncluster von einigen hundert Nanometern bis zu einigen Mikrometern im Durchmesser konnten erstmalig kontrolliert erzeugt und untersucht werden. Sie zeigen eine komplexe, oft hagelkornartige Struktur, was neue Erkenntnisse über die Morphologie und Wachstumsmechanismen von Edelgasclustern erlaubt.

In den Flugzeitmassenspektren einzelner sehr großer Xenoncluster weisen die charakteristischen Energieverteilungen der Ionen darauf hin, dass diese ausschließlich von der äußersten Oberflächenschicht der Cluster emittiert werden. Zudem finden sich Hinweise auf effektive Rekombinationsprozesse im verbleibenden, quasi-neutralen Nanoplasma, die es den allermeisten Ionen erlauben bis in den neutralen Zustand zurück zu rekombinieren.

Aus den dazugehörigen Streubildern können Intensitätsprofile extrahiert und mit Hilfe der Mie-Theorie analysiert werden. Anpassungsrechnungen liefern die optischen Eigenschaften der Cluster. In den Intensitätsprofilen von sehr großen Clustern entwickeln sich mit steigender Intensität Modulationen. Diese deuten auf die Bildung eines Nanoplasmas mit schichtartigem Aufbau hin. Der Kern und eine bis zu $50 \mathrm{~nm}$ dicke äußeren Schale unterscheiden sich in den optischen Eigenschaften, was auf ultraschnelle Änderungen der elektronischen Struktur der Cluster schließen lässt. 


\section{Contents}

1 Introduction 1

2 Theoretical concepts $\quad 5$

2.1 Propagation, absorption and scattering of light in matter . . . . . . 6

2.1.1 Wavelength dependent response of matter . . . . . . . . . . . 6

2.1.2 Scattering from free and bound electrons . . . . . . . . . . 8

2.1.3 Scattering from a spherical particle . . . . . . . . . . . . . . . . . 14

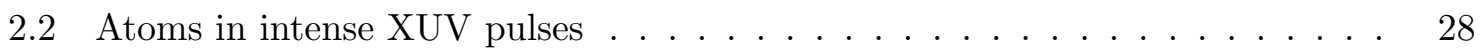

2.2.1 Wavelength dependent nonlinear processes . . . . . . . . . . 28

2.2.2 Ionization properties of atomic xenon in the XUV range . . . . . . . 32

2.3 Clusters in intense laser pulses $\ldots \ldots \ldots \ldots \ldots$

2.3.1 Clusters as model systems for laser-matter interaction . . . . . . . . . 36

2.3 .2 Properties and dynamics of a nanoplasma . . . . . . . . . . . . 37

2.3.3 Rare gas clusters in intense short-wavelength pulses . . . . . . . . . 42

$\begin{array}{lll}3 & \text { Experimental setup } & 51\end{array}$

3.1 FLASH Free-electron laser for short wavelength radiation . . . . . . . . . . 51

3.1.1 Basic principle of a free-electron laser . . . . . . . . . . . 52

3.1 .2 Characteristics of the FLASH FEL . . . . . . . . . . . 55

3.1.3 Guiding and focussing optics for XUV light . . . . . . . . . . . . 56

3.2 Experiment for imaging and ion spectroscopy of single clusters . . . . . . . 59

$3.2 .1 \quad$ Experimental layout . . . . . . . . . . . . . . . . . . 59

3.2 .2 Cluster generation . . . . . . . . . . . . . . . . 60

3.2 .3 Detection of scattered light $\ldots \ldots \ldots \ldots$. . . . . . . . 65

3.2 .4 Ion detection $\ldots \ldots \ldots \ldots \ldots 6 \ldots$ 


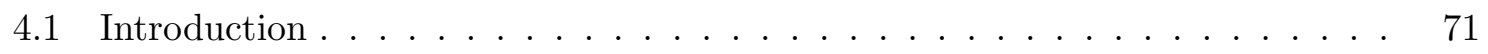

4.2 Measurements in single-particle mode . . . . . . . . . . . . . . . 73

4.2.1 Statistics in single-particle mode . . . . . . . . . . . . . . . . . 73

4.2 .2 Data acquisition and processing . . . . . . . . . . . . 74

4.2.3 The limit of single particle mode: Newton rings . . . . . . . . . . . 75

4.3 Information on cluster morphology . . . . . . . . . . . . 77

4.3.1 The shape of clusters changing with size . . . . . . . . . . 77

4.3 .2 Time structure of a pulsed jet . . . . . . . . . . 83

4.4 Ejection of surface ions from a quasi-neutral nanoplasma . . . . . . . . . . 88

4.4.1 The concept of exposure power density (epd) _ . . . . . . . . . . 89

4.4.2 SIMION simulation of the bipolar time-of-flight spectrometer . . . . . 89

4.4.3 Size dependent effects in kinetic energy distributions . . . . . . . . . 95

4.4.4 Recombination versus expansion in a large dense nanoplasma . . . . . 98

4.4.5 Net charge versus charge separation at the surface . . . . . . . . . . 100

4.5 Fingerprints of a nanoplasma shell . . . . . . . . . . . . . 105

4.5.1 Modulation in size-sorted scattering profiles . . . . . . . . . . . 106

4.5.2 Time-binning of the nanoplasma development . . . . . . . . . . . . . 108

4.5.3 Simulation of scattering profiles from core-shell systems . . . . . . . . 111

4.5.4 Optical properties of a dense xenon nanoplasma . . . . . . . . 115

4.5.5 Simulation of radial charge state densities . . . . . . . . . . . . 118

4.5.6 Conclusions for the scattering data and discussion . . . . . . . . 123

5 Summary and Outlook $\quad 125$

$\begin{array}{ll}\text { Appendix } & 127\end{array}$

$\begin{array}{ll}\text { List of figures } & 138\end{array}$

$\begin{array}{lr}\text { Bibliography } & 143\end{array}$ 


\section{Chapter 1}

\section{Introduction}

The rapid progress in laser technology over the last decades enabled pioneering insight into the timescales of chemical processes [2]. Well controlled short and intense laser pulses can be produced with state-of-the-art laser systems, which allow for temporally resolving nuclear motion within molecules and even the dynamics of valence electrons. These possibilities have initiated the new fields of femtochemistry [3] and attosecond science [4].

On the other hand the understanding of the fundamental organization of matter has been revolutionized by X-ray science since the discovery of X-rays in 1895 [5]. Groundbreaking insight into the electronic properties of matter has been achieved with core-level spectroscopy [6] and X-ray diffraction based techniques have been instrumented to investigate the structural composition of matter $[7,8]$. Structural analysis with X-rays increasingly gained relevance in life-sciences. Knowing the structure of biologically important molecules such as hemoglobin [9] and bio-chemical substances as penicillin [10] constitutes the basis for understanding their biological function. With the development of modern X-ray synchrotron sources structure determination of crystallized macromolecules has become a well-established method [11, 12]. However, the crystallization of the target molecules still poses a challenging and in many cases unsurmountable obstacle for structural analysis by X-ray diffraction [13]. Therefore, only a small fraction of biologically important systems have been structurally determined yet.

The advent of short wavelength free-electron lasers (FELs) combines femtosecond time resolution with the power of X-ray based techniques $[14,15,16]$. They deliver highly intense ultrashort pulses and photon energies well into the hard X-ray regime. One of the challenging future visions in the context of this development is the idea of imaging individual molecules in single light pulses and accessing processes in these molecules on the atomic length and time scale.

Fig. 1.1 a illustrates the concept of imaging a single molecule in flight, also referred to as diffraction before destruction [17]. A large number of photons is necessary to produce sufficient elastically scattered light for acquiring a scattering pattern. Therefore the molecule gets ultimately destroyed in the process [18]. This results in demanding requirements on the pulse duration [18]. Even if the light pulse is short enough to avoid blurring of the diffraction pattern by nuclear motion, ultrafast changes in the electronic structure will occur on a much faster time scale and may alter the information in the scattering pattern.

The availability of ultrashort intense pulses in the vacuum-ultraviolet (VUV) and extremeultraviolet (XUV) spectral regime at the Free-Electron Laser in Hamburg FLASH and up to the X-ray range at the Linear Coherent Light Source LCLS in Stanford enabled a large number of novel experiments $[19,20]$. Within this progress, single shot imaging of free parti- 

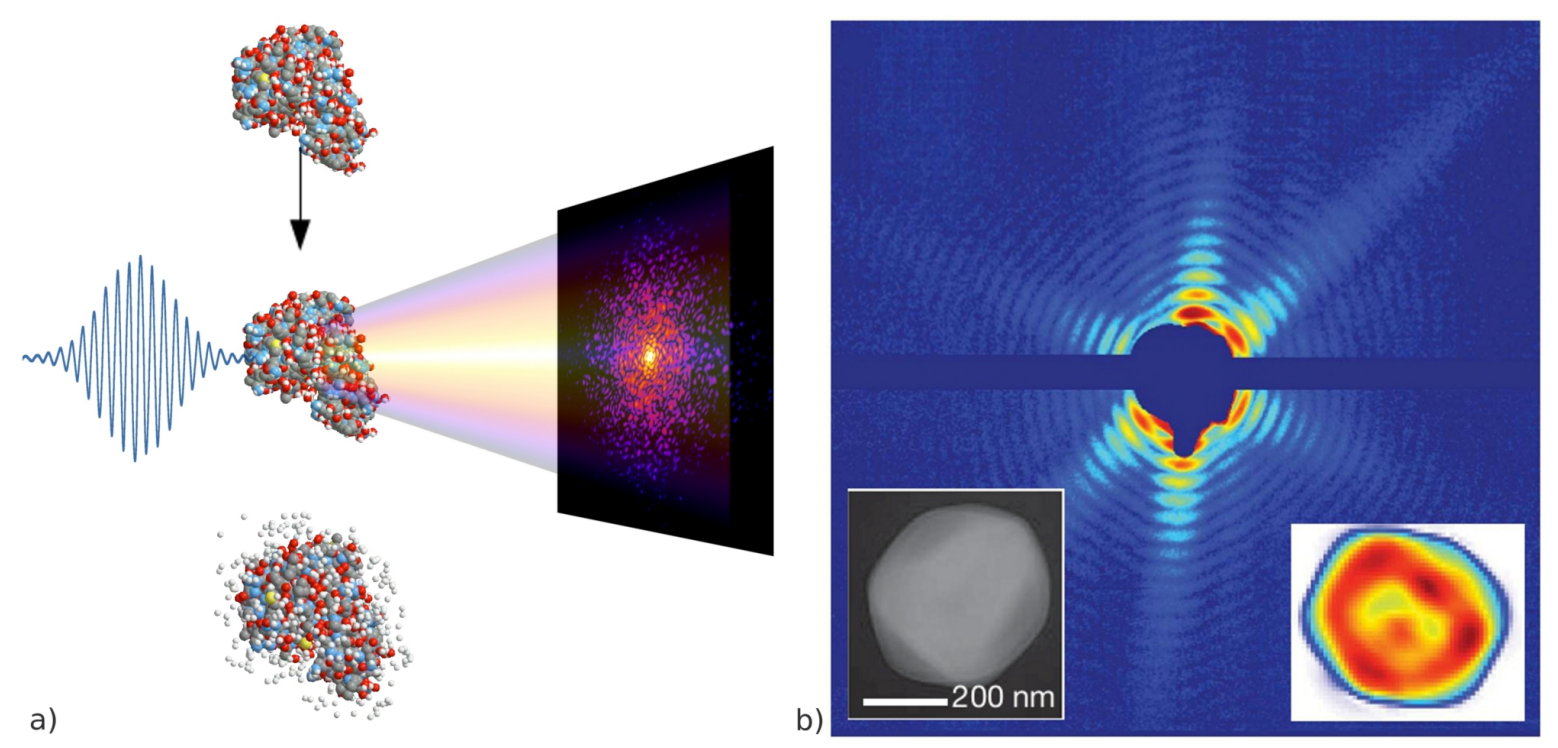

Figure 1.1: a) Concept of single-molecule imaging. An individual molecule is hit by an intense short $\mathrm{X}$-ray pulse and the resulting scattering pattern is recorded with an X-ray photon detector. The diffraction pattern has to be obtained before the molecule is destroyed. The radiation damage puts severe requirements to the duration of the X-ray laser pulse. The exploding molecule is taken from [18]. b) Diffraction pattern of a single mimivirus obtained at LCLS. The inset on the right side shows a reconstruction of the virus from the scattering pattern. For comparison, an electron microscopy image of a mimivirus is displayed in the left inset [25].

cles in the gas phase $[21,22,23,24]$ and particularly of non-repetitive and non-reproducible biological objects became possible [25]. Fig. $1.1 \mathrm{~b}$ displays the diffraction pattern of an individual virus imaged with an X-ray pulse from LCLS [25]. The inset on the right hand side shows a reconstruction of the virus, which could be calculated from the scattering pattern. For comparison, an electron microscopy image of this type of virus is displayed in the left inset. According to detailed theoretical work, the achievable spatial resolution for structure determination in such experiments is ultimately limited by radiation damage [26, 27, 28]. Hence, the understanding of underlying processes such as ionization and fragmentation is of a key interest for all kinds of imaging applications.

The investigation of rare gas clusters in the gas phase as simple model systems for the interaction of matter with intense short-wavelength pulses helps to gain deep insight in the underlying ionization processes as well as electron and nuclear dynamics [22, 29]. So far, studies on rare gas clusters have shown that the initial ionization, which denotes the first step of radiation damage, is influenced by the atomic environment [30]. Concepts for relaxing the severe conditions on pulse durations for imaging of single molecules by embedding the particles in a temper layer could be demonstrated with clusters [31]. From the scattering patterns of single clusters, both the structure and transient changes in the electronic properties of the particles due to the interaction with the light could be extracted [32]. This yields valuable insight into radiation damage evolving on the timescale of femtoseconds.

In this work the ionization and plasma dynamics of clusters in highly intense XUV pulses from the FLASH free-electron laser are investigated in a combined imaging and spectroscopy approach. By analyzing the scattering patterns the single shot data can be down-selected to ideal data sets with a well known cluster size and a defined exposure power density. The structural information from the images of single clusters yields unique insight into the growth process of rare gas clusters in a gas expansion. The findings also allow for extending 
the accessible cluster sizes up to several micrometers in diameter, by far exceeding common scaling laws [33].

The focus of this thesis is set on analyzing the scattering patterns and ion spectra in this so far unexplored size range in order to investigate the role of the nanoplasma which evolves in the clusters during the interaction with the laser pulse. The questions asked are in particular:

- What is the effect of the plasma electrons that are bound to the cluster as a whole on the scattering process?

- How does the ionization dynamics proceed in large clusters, what does the charge distribution look like?

- How is the fragmentation of the clusters influenced by the distribution of ions and the density and temperature of the electron cloud?

The results from the scattering patterns show indications of electronic rearrangements within the highly ionized nanoplasma to a core-shell type structure on a sub-100 fs time scale. In contrast the ion spectra reveal that on a much longer time scale only the outermost atomic layer of the cluster explodes off. The largest part of the cluster, even though highly ionized during the pulse, recombines to neutral atoms.

In those findings the capability of simultaneous imaging and ion spectroscopy of single clusters becomes apparent: That is to address different timescales of complex dynamics within well characterized systems and therefore gain unprecedented insight into the underlying processes. 


\section{Chapter 2}

\section{Theoretical concepts}

The investigation of the interaction between intense laser pulses and clusters is related to several different research fields. The interpretation of the light-induced processes and dynamics in finite-size particles requires concepts from atomic, solid state, and plasma physics as well as linear and nonlinear optics. In this chapter, a brief overview shall be given on the theoretical basics, which were used for analyzing and interpreting the experimental results in this work.

In the first part, the propagation of light in matter and the basics of light scattering will be discussed via a classical approach. Microscopic and macroscopic descriptions can be used to gain an understanding of the phenomena of scattering from free and bound electrons and the diffraction of light from homogenous and coated spherical particles.

The second part is dedicated to the special circumstances in this experiment introduced by the intense XUV light. While the classical descriptions imply a purely linear response of the analyzed systems, the transition towards a nonlinear response of matter has to be considered. In particular atomic processes at different laser intensities and excitation energies will be discussed. Within the perturbative regime of the experiments in this work, the element-specific response of the target material to the excitation energy is of great importance. Accordingly, the ionization properties of atomic xenon and its ions will be presented.

The phenomena related to the specific characteristics of clusters resulting from finite-size, many-body and collective effects are addressed in the third part of this chapter. The wavelength dependent excitation processes in clusters are discussed, with an emphasis on the emergence and development of a nanoplasma. In this context, helpful concepts from plasma physics are introduced. An overview is given on selected findings from laser-cluster experiments ranging from the VUV up to the X-ray spectral regime.

It is important to note, that in contrast to the clear limits assigned to the spectral regimes of infrared and visible light, deviations exist in the literature on the terminology used for higher photon energy regimes. Throughout this thesis, the photon energy of $91 \mathrm{eV}$ used in the experiment will be assigned to the extreme ultraviolet (XUV) range, which can be defined to cover energies from 30 to $100 \mathrm{eV}$. Smaller photon energies around $10 \mathrm{eV}$ will be denoted as vacuum ultraviolet (VUV), while the range above $100 \mathrm{eV}$ up to $1 \mathrm{keV}$ is referred to as soft X-ray. 


\subsection{Propagation, absorption and scattering of light in matter}

The theoretical description of light-matter interaction ranges from classical over semi-classical approaches to quantum-electrodynamics. Even though several important quantities such as light induced transition rates between atomic states can only be correctly described quantummechanically, the classical approach enables a fundamental and intuitive understanding in particular for phenomena of elastic light scattering. Therefore, the discussion in this section is restricted to a purely classical picture, departing from the basic equations of electrodynamics, the Maxwell equations. A microscopic model of the atomic systems is given by a Lorentz-like harmonic oscillator with a single resonance frequency for electrons bound to atoms.

The derivation of important relationships will be limited to the motivation of key steps. Only in the case of scattering from a spherical particle in the framework of Mie-theory (section 2.1.3), explicit expressions are given, which are later used in simulation algorithms for the analysis of experimental data.

The line of arguments follows the books of Hau-Riege, High-Intensity X-rays - Interaction with Matter [34], Attwood, Soft x-ray and extreme ultraviolet radiation [35] and Bohren and Huffmann, Absorption and Scattering of Light by Small Particles [36].

\subsubsection{Wavelength dependent response of matter}

The propagation of light in matter and the interaction between both treated in a classical picture is located in the field of optics, which can be considered a subdivision of electrodynamics. Therefore, the point of departure for considering elastic light scattering can be found in the basic equations of electrodynamics. The Maxwell equations [37] connect the electric and magnetic fields to each other and to their sources, charge and current. In MKS units they read [38]

$$
\begin{array}{rlrl}
\vec{\nabla} \cdot \vec{D} & =\rho & & \text { (Coulomb's law) } \\
\vec{\nabla} \cdot \vec{B} & =0 & \\
\vec{\nabla} \times \vec{E} & =-\frac{\partial \vec{B}}{\partial t} & & \text { (Faraday's law) } \\
\vec{\nabla} \times \vec{H} & =\vec{J}+\frac{\partial \vec{D}}{\partial t}, & & \text { (Ampere's law) }
\end{array}
$$

where $\rho$ is the charge density, $\vec{D}$ is the electric displacement and $\vec{B}$ is the magnetic induction. The electric field vector is denoted as $\vec{E}$, the magnetic field vector is given by $\vec{H}$, and $\vec{J}$ is the current density. The influence of an electromagnetic field on matter and its response are defined by the constitutive relations

$$
\begin{aligned}
\vec{D} & =\varepsilon_{0} \vec{E}+\vec{P}(\vec{E}) \\
\vec{H} & =\frac{1}{\mu_{0}} \vec{B}+\vec{M}(\vec{H})
\end{aligned}
$$

with $\vec{P}$ being the electric polarization and $\vec{M}$ the magnetic polarization, respectively. The constants $\varepsilon_{0}$ and $\mu_{0}$ denote the permittivity and the magnetic permeability of free space. In the absence of matter, the constitutive relations reduce to

$$
\begin{aligned}
\vec{D} & =\varepsilon_{0} \vec{E} \\
\vec{B} & =\mu_{0} \vec{H} .
\end{aligned}
$$


An important relationship between both matter-specific source terms, charge and current density, can be directly derived from the Maxwell equations. By taking the divergence of Ampere's law, equation 2.4 and recalling that $\nabla(\nabla \times \vec{a})$ equals zero, the so called charge continuity equation is derived:

$$
\nabla \vec{J}+\frac{\partial \rho}{\partial t}=0
$$

From another combination of Maxwell equations the vector wave equations arise. Equations 2.1 to 2.4 together with recognizing the constitutive equations 2.5 for propagation in a material yield

$$
\left(\frac{\partial^{2}}{\partial t^{2}}-c^{2} \nabla^{2}\right) \vec{E}(\vec{r}, t)=-\frac{1}{\varepsilon_{0}}\left[\frac{\partial \vec{J}(\vec{r}, t)}{\partial t}+c^{2} \nabla \rho(\vec{r}, t)\right] .
$$

In the case of free space the charge density and current density are absent, thus the second part of the vector wave equation will be zero. The solution of these equations for several specific source terms will be the focus of discussion in the next sections.

The constant $c$, which is given by $c=1 / \sqrt{\varepsilon_{0} \mu_{0}}$, can be identified as the phase velocity of an electromagnetic wave in vacuum. For propagation in matter the phase velocity $v_{p}$ is found to be dispersive. Therefore, an important quantity can be introduced, the complex refractive index $n$ of a material with $v_{p}=\frac{c}{n}$. Especially in the XUV, where the refractive index tends to differ only slightly from unity, $n$ is typically expressed as

$$
n=n^{\prime}+i \cdot n^{\prime \prime}=1-\delta+i \cdot \beta .
$$

The role of the imaginary and real part of the refractive index can be illustrated by considering a plane wave of the form

$$
\vec{E}(\vec{r}, t)=\vec{E}_{0} e^{i(\vec{k} \vec{r}-\omega t)}
$$

Note that plane waves are solutions of the vector wave equation 2.8 , even though they can not be considered physical fields as they are not normalizable. However, physically appropriate wave packages can be Fourier synthesized by plane waves. This will be further used in the context of Mie theory in section 2.1.3.

The phase velocity connects the wave vector $\vec{k}=k \hat{k}$ with the refractive index by $v=\omega / k=$ $c /(1-\delta+i \beta)$, therefore a plane wave propagating through a material with refractive index $n=1-\delta+i \beta$ yields

$$
\vec{E}(\vec{r}, t)=\underbrace{\vec{E}_{0} e^{-i \omega(t-r / c)}}_{\text {vacuum propagation }} \cdot \underbrace{e^{-i(2 \pi \delta / \lambda) r}}_{\text {phase shift }} \cdot \underbrace{e^{-(2 \pi \beta / \lambda) r}}_{\text {decay }} .
$$

While the real-valued part of the refractive index induces a phase shift to the wave compared to the propagation in vacuum, which results for example in the phenomena of refraction, the imaginary part is responsible for a decay of the field amplitude and therefore a measure of absorption in matter. The observable accessible in experiments is not the electric field vector but the intensity $I$ given by the square of the amplitude $I=|\vec{E}|^{2}$. In direction of propagation $\hat{z}$ through a material with refractive index $n=1-\delta+i \beta$, the intensity is given by

$$
I(z)=\left|\vec{E}_{0}\right|^{2} \cdot e^{\frac{4 \pi \beta}{\lambda} z} \text {. }
$$

The decay of the intensity to $1 / e$ of the initial value defines the so called penetration depth

$$
l_{a}=\frac{\lambda}{4 \pi \beta} .
$$


Real and imaginary part of the refractive index do not vary independently, they are related to each other by the so called Kramers-Kronig dispersions relations [38, 39]. They read:

$$
\begin{aligned}
& \delta(\omega)=\frac{2}{\pi} P_{C} \int_{0}^{\infty} d \Omega \frac{\Omega \cdot \beta(\Omega)}{\Omega^{2}-\omega^{2}}, \\
& \beta(\omega)=\frac{-2 \omega}{\pi} P_{C} \int_{0}^{\infty} d \Omega \frac{\delta(\Omega)}{\Omega^{2}-\omega^{2}} .
\end{aligned}
$$

$P_{C}$ denotes the value of the Cauchy integrals around the pole points in the denominator. It is important to note that dispersion relations as Eq. 2.12 also connect the real and imaginary part of other possible representations of the optical properties of matter, such as atomic scattering factors $f(\omega)$ or dielectric function $\epsilon(\omega)$ which will be introduced below. The KramersKronig relation are in practice used for calculating one quantity from the measurement of the other over a large spectral range (cf. section 4.5.4).

In order to gain a deeper understanding of light scattering, it is important to set macroscopical observation quantities as $\beta$ and $\delta$ in the context of microscopic processes. Therefore, the microscopical process of light scattering can be described as a periodic motion of charges in a time varying electric field, which leads to radiation. Considering the scattering from free and bound electrons as discussed in the next section 2.1.2 is able to complement the diffraction from extended spherical particles, treated subsequently in section 2.1.3, which is in particular important for the interpretation of the current results.

\subsubsection{Scattering from free and bound electrons}

Elastic light scattering denotes the process of redirecting photons without changing their energy. To some extend, elastic light scattering can only be an idealized process, as photons also possess momentum. Only for collisions with particles of infinite mass, totally elastic processes can be assumed, which becomes particularly important in the X-ray regime. However, in the XUV range around $100 \mathrm{eV}$ photon energy, the recoil of the photons can still be neglected [34].

Scattering of electromagnetic waves is always a phenomenon of inhomogeneities in the path of light propagation [36]. Inhomogeneities which induce light scattering can be found on the atomic scale or on the scale of aggregation of many atoms, such as interfaces or density fluctuations. Extended homogeneous media do not induce light scattering.

As a classical model of the microscopic scattering process radiation from accelerated point charges is considered in this section. In order to mimic the bound state of electrons in atoms, a Lorentzian model of a single-resonance oscillator can be employed. The description is limited to a linear response of matter to light and therefore to low intensities of the light field. Nonlinear effects in intense light fields will be discussed in section 2.2.

As a first step the radiation field from an accelerated point charge will be calculated. To quantify the ability of the electrons to scatter light, the concept of scattering cross-sections is introduced. Subsequently, the cross-sections of free and bound electrons as well as multielectron atoms can be derived.

Electrical field of an oscillating charge: The vector wave equation 2.8 has been derived above from the Maxwell equations in a representation which explicitly denotes the 
dependencies from the matter-related sources of the fields, the charge and current densities:

$$
\left(\frac{\partial^{2}}{\partial t^{2}}-c^{2} \nabla^{2}\right) \vec{E}(\vec{r}, t)=-\frac{1}{\varepsilon_{0}}\left[\frac{\partial \vec{J}(\vec{r}, t)}{\partial t}+c^{2} \nabla \rho(\vec{r}, t)\right]
$$

Only the wave equation for $\vec{E}$ is given here, and also the subsequent discussion concentrates on the description of the electric field. In principle, analog derivations could be conducted for $\vec{B}$. However, the acceleration of the electrons from the $\vec{B}$-component of the light field can be neglected according to the following considerations.

A charge in an incident light wave $\left(\vec{E}_{i n c}, \vec{B}_{i n c}\right)$ is driven by the Lorentz force

$$
\vec{F}=m \vec{a}=-e\left[\vec{E}_{i n c}+\vec{v} \times \vec{B}_{i n c}\right] .
$$

As long as $v \ll c$ the acceleration results mainly from the electric field because the magnetic field is proportional to $\vec{B}_{i n c} \propto \frac{v}{c} \vec{E}_{i n c}$.

The electric field which will be radiated by an arbitrary oscillating charge can be calculated from equation 2.13. In order to simplify the derivation, a mathematical trick is employed by transforming fields and source terms into the frequency domain $[35,38]$. The operators $\partial / \partial t$ and $\nabla$ transform to $-i \omega$ and $i \vec{k}$, respectively. All quantities in the spatial domain $A(\vec{r}, t)$ can be expressed by their Fourier transforms

$$
A(\vec{r}, t)=\int_{\vec{k}} \int_{\omega} A_{k \omega} e^{-i(\omega t-\vec{k} \vec{r})} \frac{d \omega d \vec{k}}{(2 \pi)^{4}}
$$

Then, by recognizing the operator transfers, the electrical field from Eq. 2.13 transforms to

$$
\left(\omega^{2}-k^{2} c^{2}\right) \vec{E}_{k \omega}=\frac{1}{\varepsilon_{0}}\left[(-i \omega) \vec{J}_{k \omega}+i c^{2} \vec{k} \rho_{k \omega}\right] .
$$

The charge continuity equation 2.7 reads in frequency space

$$
i \vec{k} \vec{J}_{k \omega}-i \omega \rho_{k \omega}=0
$$

with $\vec{k}=k \cdot \hat{k}_{0}$. Therefore, the Fourier transform of the field $\vec{E}_{k \omega}$ is found to be

$$
\vec{E}_{k \omega}=\frac{-i \omega}{\varepsilon_{0}}\left[\frac{\vec{J}_{k \omega}-\hat{k}_{0}\left(\hat{k}_{0} \vec{J}_{k \omega}\right)}{\omega^{2}-k^{2} c^{2}}\right] .
$$

Note, that the numerator of equation 2.16 projects the current density in frequency domain $\vec{J}_{k \omega}$ on the direction perpendicular to $\hat{k}_{0}$ :

$$
\vec{E}_{k \omega}=\frac{-i \omega}{\varepsilon_{0}} \frac{\vec{J}_{T, k \omega}}{\omega^{2}-k^{2} c^{2}} .
$$

For point radiators, such as electrons in our model, the charge density can be expressed by the Dirac delta function $\delta(\vec{r})$, which yields the current density

$$
\vec{J}(\vec{r}, t)=-e \delta(\vec{r}) \vec{v}(t)
$$

Substituted in $\vec{E}_{k \omega}$, and transformed back into space domain, the electric field radiated by an oscillating point charge can be expressed as

$$
\begin{aligned}
\vec{E}(\vec{r}, t) & =\frac{e}{4 \pi \varepsilon_{0} c^{2} r} \underbrace{\frac{d \vec{v}_{T}\left(t-\frac{r}{c}\right)}{d t}}_{\vec{a}_{T}} \\
\Leftrightarrow \vec{E}(\vec{r}, t) & =\frac{e \cdot \vec{a}_{T}\left(t-\frac{r}{c}\right)}{4 \pi \varepsilon_{0} c^{2} r} .
\end{aligned}
$$




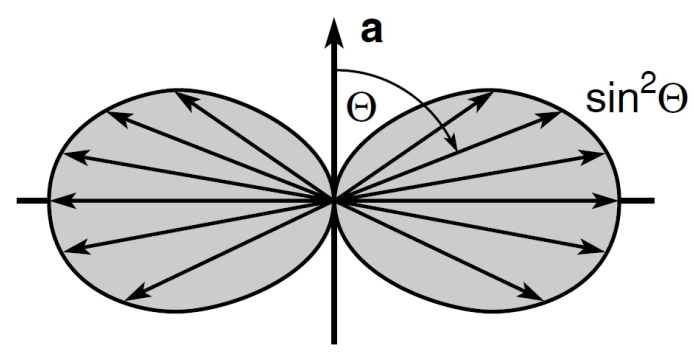

Figure 2.1: Characteristic dipole emission observed in great distance to an oscillating point charge. $\vec{a}$ denotes the acceleration vector of the charge, the emission in this direction $\hat{a}$ is zero. The emitted power under an observation angle $\theta$ is proportional to $\sin ^{2} \theta$. From [35].

This relation reveals, that the emitted field has been generated from the acceleration component of the charge perpendicular to the propagation direction, but at a retarded time $t^{\prime}=t-r / c$ needed for the light to travel to the observer. The exclusive dependency on the transverse component of the acceleration is equivalent to the well known characteristic of a dipole emitter as displayed in Fig. 2.1. In great distance to an oscillating point charge, a characteristic $\sin ^{2} \theta$-distribution of the emitted power is observed, with $\theta$ being the observation angle. In the direction of the acceleration $\hat{a}$, the emission is zero.

In order to find a measure for the ability of systems to scatter light, the incident and scattered power will be examined. The energy transport of an electromagnetic wave is represented by the Pointing vector $\vec{S}$ with

$$
\vec{S}(\vec{r}, t)=\sqrt{\frac{\varepsilon_{0}}{\mu_{0}}}|\vec{E}|^{2} \hat{k}_{0} .
$$

Therefore, the radiated power per unit area from an oscillating charge yields

$$
\vec{S}(\vec{r}, t)=\frac{e^{2} \cdot\left|\vec{a}_{T}\right|^{2}}{16 \pi^{2} \varepsilon_{0} c^{3}} \frac{\hat{k}_{0}}{r^{2}}=\text { const } \cdot \frac{\left|\vec{a}_{T}\right|^{2}}{r^{2}} \hat{k}_{0} .
$$

The radiated power from the point source decreases quadratically to the distance $r$, which corresponds to a constant flux of energy through a sphere around the point charge with increasing area $\propto r^{2}$. The total power $P$ emitted by an oscillating electron with acceleration $\vec{a}$ is found by substituting the transversal component of the acceleration $\vec{a}_{T}=\vec{a} \cdot \sin \theta$ (cf. Fig. 2.1) and integrating over the full solid angle,

$$
P=\frac{e^{2}}{6 \pi \varepsilon_{0} c^{3}}|\vec{a}|^{2} .
$$

As an important quantity for comparing the ability of systems to scatter light, the scattering cross-section $\sigma$ can be introduced. $\sigma$ is defined as the ratio of radiated to incident power

$$
\sigma=\frac{P_{\text {scatt }}}{\left|\vec{S}_{\text {inc }}\right|}
$$

In order to derive the cross-sections of free and bound electrons, the explicit form of their respective accelerations $\vec{a}$ has to be derived in the subsequent paragraphs. 
Thomson scattering from a free electron: From the Lorentz force (equation 2.14, the $v \times \vec{B}$ term will be neglected again) the acceleration of a free electron in a light field can be calculated

$$
\vec{a}(\vec{r}, t)=-\frac{e}{m_{e}} \vec{E}_{i n c}(\vec{r}, t) ; \quad \Leftrightarrow a_{T}=a \sin \theta=-\frac{e}{m_{e}} E_{i n c, 0} \sin \theta .
$$

Therefore, the radiated electric field 2.17 yields

$$
\vec{E}_{\text {scat }}(\vec{r}, t)=\frac{r_{e}}{r} E_{i n c, 0} \sin \theta e^{-i \omega\left(t-\frac{r}{c}\right)},
$$

with the electron radius $r_{e}=e^{2} /\left(4 \pi \varepsilon_{0} m_{e} c^{2}\right)=2.8 \cdot 10^{-15} \mathrm{~m}$. By calculating the total power (Eq. 2.19), the elastic scattering cross-section of a free electron, also referred to as Thomson cross-section [40] can be found:

$$
\sigma_{e l}=\frac{8 \pi}{3} r_{e}^{2}
$$

The Thomson cross-section does not depend on the frequency of the incident electromagnetic field. It has a value of about $6.7 \cdot 10^{-29} \mathrm{~m}^{2}$ or 0.67 barn. This value will be valid up to photon energies in the hard X-ray regime, where photon recoil starts to play a role.

Elastic scattering from bound electrons: In order to calculate the cross-section of an electron bound to an atom, the mechanical model of a driven oscillator with resonance frequency $\omega_{\text {res }}$ and damping $\gamma$ can be used. The response of such a system to the incident wave is dependent on the difference between the light frequency and the oscillator frequency $\left(\omega-\omega_{\text {res }}\right)$ The acceleration of the bound electron can be determined by the equation of forces

$$
m_{e} \frac{d^{2} \vec{x}}{d t^{2}}+m_{e} \gamma \frac{d \vec{x}}{d t}+m_{e} \omega_{r e s}^{2} \vec{x}=-e \vec{E}_{i n c}
$$

again neglecting the magnetic field term of the Lorentz force. Solving this differential equation yields the acceleration

$$
\vec{a}=\frac{-\omega^{2}}{\omega^{2}-\omega_{r e s}^{2}+i \gamma \omega} \frac{e \vec{E}_{i n c}}{m_{e}} .
$$

By calculating the Pointing vector 2.18, we obtain the cross-section of a bound electron:

$$
\sigma_{\text {bound }}=\frac{8 \pi}{3} r_{e}^{2} \frac{\omega^{4}}{\left(\omega^{2}-\omega_{\text {res }}^{2}\right)^{2}+(\gamma \omega)^{2}} .
$$

The strong dependency of the cross-section on the frequency is depicted in Fig. 2.2.

Far above the resonance frequency, $\omega \gg \omega_{\text {res }}$, the light induced oscillations become too fast to notice the response of the oscillator and the cross-section approaches the Thomson value. If the frequency of the light field approaches the resonance frequency of the oscillator, the cross-sections becomes maximal. The damping term $\gamma$ defines the width of the resonance. If $\omega \ll \omega_{\text {res }}$ Eq. 2.30 reduces to the well known relationship for Rayleigh scattering [41] (under the condition $\left.\gamma \ll \omega_{\text {res }}\right)$

$$
\sigma_{R}=\frac{8 \pi}{3} r_{e}^{2}\left(\frac{\omega}{\omega_{\text {res }}}\right)^{4}
$$




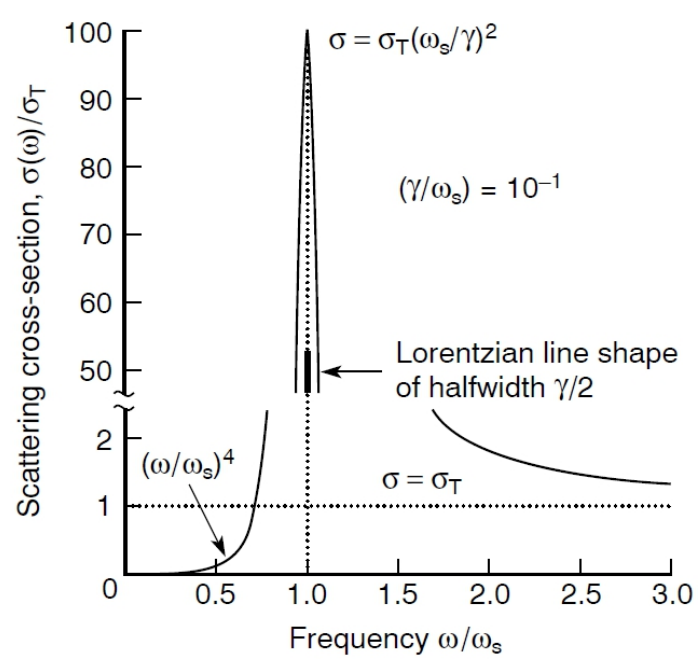

Figure 2.2: Semi-classical scattering cross-section of a bound electron with resonant frequency $\omega_{s}$ normalized to the Thomson cross-section. For much smaller frequencies than the resonant frequency, the Rayleigh-limit is observed $\left(\sigma \propto \lambda^{-4}\right)$. For much larger frequencies, the scattering cross-section of a bound electron approaches the Thomson value. From [35].

Elastic scattering from multi-electron atoms: The electrons in an atom can be tightly bound in inner electronic shells or only weakly in case of a valance shell. Therefore, a classical model of a multi-electron atom can be constructed from several oscillators with different coupling constants and thus different resonance frequencies $\omega_{s}$. In addition, for short wavelength radiation the individual coordinates $\vec{r}_{s}$ of the point charges have to be considered.

Analog to the discussion above for single electrons, the electrical field emitted by the multielectron atom can be calculated. Following the superposition principle [42], $\vec{E}(\vec{r}, t)$ will be given by the sum of the fields from each individual electron

$$
\vec{E}(\vec{r}, t)=\frac{e}{4 \pi \varepsilon_{0} c^{2}} \sum_{s=1}^{Z} \frac{\vec{a}_{T, s}\left(t-r_{s} / c\right)}{r_{s}}
$$

which can be simplified by introducing the atomic scattering factor $f(\Delta k, \omega)$,

$$
\vec{E}(\vec{r}, t)=-\frac{r_{e}}{r} \underbrace{\left[\sum_{s=1}^{Z} \frac{\omega^{2} e^{i \Delta k \Delta r_{s}}}{\omega^{2}-\omega_{s}^{2}+i \gamma \omega}\right]}_{f(\Delta k, \omega)} E_{i n c, 0} \sin \theta e^{-i \omega(t-r / c)} .
$$

In the here presented form the atomic scattering factor denotes a complicated quantity, which is dependent from the relative coordinates of the individual electrons $\Delta r_{s}$. However, the relationship can be simplified by considering that in the XUV range the wavelength of the light is still larger than the atomic dimension $\lambda \gg a_{0}$, the so called Bohr radius. As most of the electron density is located within a distance to the core approximated by $a_{0}$, all electrons will experience the same field strength. This reduces the atomic scattering factor to

$$
f(\omega)=\sum_{s=1}^{Z} \frac{\omega^{2}}{\omega^{2}-\omega_{s}^{2}+i \gamma \omega}
$$


In a multi-electron atom several electrons share the same resonance frequency, as - quantummechanically spoken - they occupy the same shell. Therefore, it is convenient to introduce a quantity referred to as oscillator strength $g_{s}$. In the classical model, $g_{s}$ simply equals the number of electrons in a shell $s$ with the same resonant frequency. The $g_{s}$ obey the sum rule $\sum_{s=1}^{Z} g_{s}=Z$, whereas $Z$ is the total number of electrons in the atom. From a quantummechanically correct treatment the $g_{i k}$ arise as transition probabilities between state i and state $\mathrm{k}$. Also for the quantum-mechanically quantity $g_{i k}$ the sum rule is valid.

From Eq. 2.28, 2.31 and 2.19 the scattering cross-section of a multi-electron atom can be obtained as

$$
\sigma=\frac{8 \pi}{3} r_{e}^{2}|f(\omega)|^{2}
$$

with the complex atomic scattering factor

$$
f(\omega)=\sum_{s} \frac{g_{s} \omega^{2}}{\omega^{2}-\omega_{s}^{2}+i \gamma \omega}=f_{1}(\omega)-i f_{2}(\omega) .
$$

The complex refractive index as the macroscopic corespondent to the atomic scattering factors can be expressed in microscopic terms by

$$
n(\omega)=1-\frac{n_{a} r_{e} \lambda^{2}}{2 \pi}\left(1-f_{1}(\omega)+i f_{2}(\omega)\right)
$$

which yields the relationships separately for real and imaginary parts

$$
\begin{aligned}
& \delta=\frac{n_{a} r_{e} \lambda^{2}}{2 \pi} f_{1}, \\
& \beta=\frac{n_{a} r_{e} \lambda^{2}}{2 \pi} f_{2} .
\end{aligned}
$$

Though the derivation of the relationship between atomic scattering factors and complex refractive index will not be explicitly presented here, it can be derived in full analogy to the previous discussion (cf. [35], p. $56 \mathrm{ff}$ ). A large number of multi-electron atoms have to be considered in extended media, but obviously the approximation of all electrons experiencing the same field is not valid here. As a result, different coordinates for all atoms have to be accounted for, which is for example responsible for the very complicated structures of diffraction patterns obtained from large bio-molecules (cf. for example Fig. 1.1).

However, in the case of homogeneously extended media, the following consideration can help to simplify the problem: In the direction of propagation, the relative coordinates of the atoms do not matter, constructive interference leads to the propagation of light in matter and reproduces well known facts such as a decreased phase velocity.

At the transition of plain interfaces the direction of constructive interference is given by the refractive index $n$, which defines a change in the propagation direction, known as refraction. For all other directions than the propagation direction, the scattered fields from all multielectron atoms in extended media will average out. This observation is equivalent to the statement at the very beginning of this section, that light scattering is a phenomenon of inhomogeneity.

Also the theory of Mie-scattering, as discussed in the next section, is based on constructive and destructive interference as a function of the observation angle of light, which is scattered from the interface of a spherical surface. 


\subsubsection{Scattering from a spherical particle}

The mathematical description of elastic light scattering from a homogeneous spherical particle with arbitrary size and optical properties has been developed by Gustav Mie in 1908 [43]. Mie wanted to explain the various colors observed from a solvation of small gold colloids. Since then, a multitude of optical phenomena arising in connection with small particles have been analyzed using Mie's theory [36].

On the one hand, the individual rare gas clusters studied in this thesis are free particles in the vacuum with an interface between solid density and vacuum surrounding with the thickness of an atomic layer. With these properties, they constitute an ideal realization of the constraint of a homogeneous sphere in a non-absorbing medium.

On the other hand, Mie's theory represents a purely static approach while the optical properties of the clusters will change during the interaction with an intense laser pulse [32]. Even though great progress has been made in modeling the microscopic processes in a time resolved manner up to particle sizes of about $10^{6}$ atoms [44], the large clusters studied in the here presented experiment exceeding $10^{8}$ atoms are still out of reach for attempting a dynamical microscopic analysis. A detailed discussion of the temporal evolution of the clusters exposed to laser pulses will be presented in the last part of this chapter 2.3.

Approximating the evolving clusters by static homogeneous particles with average optical properties allows for a macroscopic approach within the framework of Mie's theory for analysis of the scattering patterns. From the Mie formalism the intensity scattered from a sphere with distinct optical properties in dependency of the observation angle can be calculated.

By fitting the experimentally obtained scattering patterns of single clusters with calculated ones, access is gained to the size and refractive index of the particle as well as to the intensity of the incident light field [36]. Changes in the refractive index found in scattering patterns of clusters exposed to different incident intensities can then be related to the evolving electronic properties of the cluster within the interaction with the pulse [32].

In particular for cluster sizes considerably exceeding the wavelength and the penetration depth of the light, as present in the current experiment, radial variations in the optical properties occur (cf. chapter 4.5). An approach to access information of such radially changing electronic properties in the particles can be made within Mie's theory by extending the formalism to coated spheres [45].

Both, scattering from a homogeneous and a coated sphere can be derived analytically from the Maxwell equations. Only the key steps of the derivation are outlined in this chapter. But as the experimental data have been analyzed with Mie-based algorithms, some explicit expressions - for example of the scattering coefficients which are calculated by the algorithms - are presented in spite of their extensive form.

Unless otherwise specified, the derivations presented in this section follow the description given in Bohren and Huffman [36], chapters 2, 3 and 4.

Boundary conditions: Mie's theory is the analytic solution of the Maxwell equations for the geometrical problem of a spherical particle in a non-absorbing medium. Fig. 2.3 displays the geometry and the notations for the fields in the distinct areas. Inside the sphere, the fields are denoted $\vec{E}_{\text {sphere }}, \vec{H}_{\text {sphere }}$. The fields in the region outside the sphere $\vec{E}_{\text {out }}, \vec{H}_{\text {out }}$ equal the sum of incident and scattered fields

$$
\vec{E}_{\text {out }}=\vec{E}_{\text {inc }}+\vec{E}_{\text {scat }} \quad \vec{H}_{\text {out }}=\vec{H}_{\text {inc }}+\vec{H}_{\text {scat }}
$$




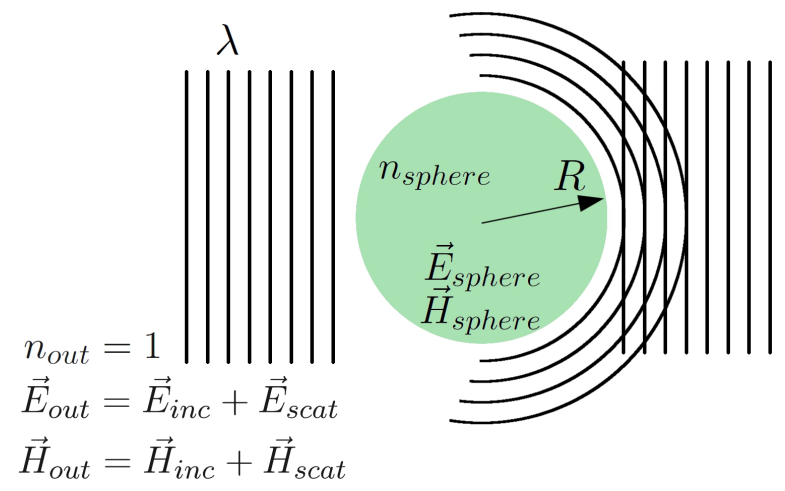

Figure 2.3: Geometry and fields of the simple case of light scattering from a sphere. A homogeneous sphere with radius $R$ and complex refractive index $n_{\text {sphere }}$ is surrounded by vacuum $(n=1)$. The fields inside the sphere are denoted as $E_{\text {sphere }}, H_{\text {sphere }}$, the fields outside the sphere $E_{\text {sphere }}, H_{\text {sphere }}$ consist in the sum of incident and scattered fields.

Basically two constraints define the analytically solvable problem of Mie's theory. Incident, inner and scattered fields have to

- be a solution of the Maxwell equations and

- fulfill boundary conditions at the surface of the sphere.

In order to find solutions of the Maxwell equations which satisfy the spherical geometry of the problem, a more compact representation of the vector wave equations has to be be chosen. The source terms, charge and current density, which have been expressed explicitly on the right side of equation 2.8 for the purpose of a microscopical analysis of the scattering process, will be further on implicitly contained on the left side.

By restricting the considered media to be homogeneous, linear and isotropic, the connections between field and polarization can be expressed by linear susceptibilities $\chi_{e l}$ and $\chi_{m a g}$. Then the magnetic permeability $\mu$ and the permittivity $\varepsilon$ can be introduced, which incorporate the material properties

$$
\mu=\mu_{0}\left(1+\chi_{m a g}\right), \quad \varepsilon=\varepsilon_{0}\left(1+\chi_{e l}\right)+i \frac{\sigma}{\omega} .
$$

In addition, the considerations will be restricted to time-harmonic fields of the form $(\vec{F}=\vec{E}$ or $\vec{H}$ )

$$
\vec{F}_{c}=\vec{C} \cdot e^{-i \omega t}
$$

Formally, this corresponds to a transformation into the frequency domain, which has been described before in section 2.1.2. Time-harmonic fields and in particular plain waves are able to simplify the solutions of the vector wave equations and can be eventually used to Fourier synthesize arbitrary wave packages.

The vector wave equations are thereby reduced to

$$
\begin{aligned}
\vec{\nabla}^{2} \vec{E}+k^{2} \vec{E} & =0 \\
\vec{\nabla}^{2} \vec{H}+k^{2} \vec{H} & =0,
\end{aligned}
$$

with the dispersion relation

$$
k^{2}=\omega^{2} \varepsilon \mu=\frac{\omega^{2} n^{2}}{c^{2}} .
$$




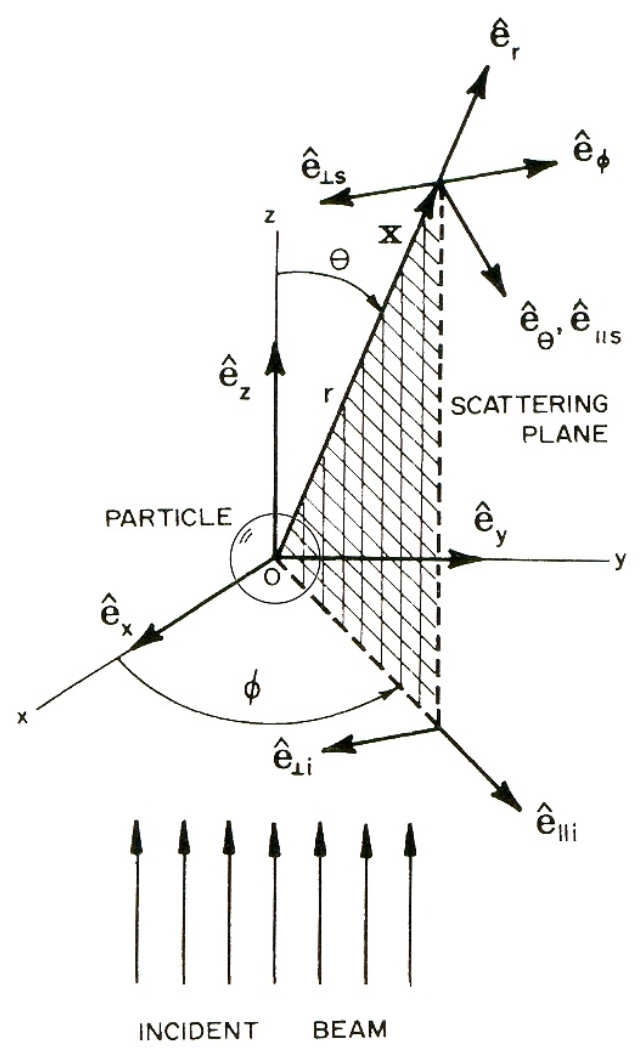

Figure 2.4: Coordinate systems for incident and scattered waves. The propagation direction of the incident wave is defined as $\hat{z}$, which spans the scattering plane together with the propagation direction $\hat{k}$ of the scattered wave. From [36].

The boundary conditions are given by the demand for energy conservation at the discontinuity of the sphere surface. This is equivalent to the conservation of the tangential components of the fields at all points $\vec{x}_{S}$ on the surface,

$$
\begin{array}{r}
{\left[\vec{E}_{\text {out }}\left(\vec{x}_{S}\right)-\vec{E}_{\text {sphere }}\left(\vec{x}_{S}\right)\right] \times \hat{r}=0} \\
{\left[\vec{H}_{\text {out }}\left(\vec{x}_{S}\right)-\vec{H}_{\text {sphere }}\left(\vec{x}_{S}\right)\right] \times \hat{r}=0 .}
\end{array}
$$

If the boundary conditions 2.38 are fulfilled, the integral of the pointing vectors $\oint \vec{S}_{\text {out }}$ and $\oint \vec{S}_{\text {sphere }}$ over the surface area will be equal, which ensures energy conservation.

Amplitude scattering matrix: The transformation between incident and scattered wave can be described by a linear operator, the so called amplitude scattering matrix. In order to find appropriate basis sets for the incident and scattered fields, two different coordinate systems are introduced. They are displayed in Fig. 2.4 [36]. The propagation direction of the incident wave is set to the $\hat{z}$ direction per definition. Together, the propagation directions $\hat{k}$ of incident and scattered wave span the scattering plane. $\hat{k}$ of the scattered wave defines the observation direction. Therefore, the scattering plane has to be defined anew for changing observation directions.

Incident and scattered waves can be projected on the scattering plane and split up into perpendicular and parallel components, respectively. The incident field is considered a plane wave. The scattered field in the vicinity of the scatterer will be a spherical wave, therefore a spherical coordinates system $\left(\hat{e}_{r}, \hat{e}_{\Theta}, \hat{e}_{\Phi}\right)$ is introduced. However, in the limit of the far field 
$(k r \gg 1)$ the scattered wave will be also approximately transverse and can be described as

$$
\vec{E}_{\text {scat }}=\frac{e^{i k r}}{-i k r} \vec{E}_{\text {scat }, f a r}
$$

with $\vec{E}_{\text {scat }, f a r} \cdot \hat{e}_{r}=0$. The two different coordinate systems for the incident and scattered wave indicated in Fig. 2.4, correspond to the representation of the fields $\vec{E}_{\text {inc }}$ and $\vec{E}_{\text {scat }}$ in different basis sets. However, due to the linearity of the boundary conditions 2.38, linear transformations between incident and scattered fields can be found.

In optics, similar linear transformations are known as Johnson matrices [38], which are used as a mathematical description of optical components, such as focussing of light with a lens or changing its polarization with a polarization filter. The linear transformation matrix which describes the scattering process is referred to as amplitude scattering matrix. With the amplitude scattering matrix the relationship between incident and scattered fields can be written as

$$
\left(\begin{array}{c}
E_{\| s} \\
E_{\perp s}
\end{array}\right)=\frac{e^{i k(r-z)}}{-i k r}\left(\begin{array}{cc}
S_{2} & S_{3} \\
S_{4} & S_{1}
\end{array}\right)\left(\begin{array}{c}
E_{\| i} \\
E_{\perp i}
\end{array}\right) .
$$

In order to calculate scattering patterns of spherical scatterers with different size and material constants, angular dependent expressions of the elements $S_{i}$ of the amplitude scattering matrix have to be found.

Solving the vector wave equations: The wave equations which follow directly from the Maxwell equations and which have to be fulfilled by the incident and scattered fields are vector wave equations. In other words, they do not apply individually to each component of the field vectors, such as $\partial^{2} E_{x} / \partial x^{2}+k^{2} E_{x}=0$, which would be easy to solve. Nevertheless, a basis system of vector functions $\vec{M}, \vec{N}$ can be found, which reduces the vector wave equations to scalar wave equations. The vector harmonics are defined as

$$
\vec{M}=\vec{\nabla} \times(\vec{c} \cdot \Psi) \quad \vec{N}=\frac{\vec{\nabla} \times \vec{M}}{k}
$$

with the guiding vector $\vec{c}$ and the scalar generation function $\Psi$.

It can be easily tested that they solve the Maxwell equations: For both $\vec{M}$ and $\vec{N}$, the divergence equals zero. Further, the rotation of $\vec{M}$ yields $k \vec{N}$ and vice versa.

If inserted in the vector wave equation they lead to

$$
\vec{\nabla}^{2} \vec{M}+k^{2} \vec{M}=\vec{\nabla} \times\left[\vec{c}\left(\vec{\nabla}^{2} \Psi+k^{2} \Psi\right)\right]
$$

As the right-hand side of this equation has to be zero, the problem can be reduced to solve the scalar equation

$$
\vec{\nabla}^{2} \Psi+k^{2} \Psi=0
$$

According to the spherical symmetry of the problem, the guiding vector $\vec{c}$ is set to $\vec{r}$ which yields the spherical vector harmonics. Also the generation function $\Psi$ will be adapted to spherical symmetry. In spherical coordinates the scalar wave equation reads

$$
\frac{1}{r^{2}} \frac{\partial}{\partial r}\left(r^{2} \frac{\partial \Psi}{\partial r}\right)+\frac{1}{r^{2} \sin \theta} \frac{\partial}{\partial \theta}\left(\sin \theta \frac{\partial \Psi}{\partial \theta}\right)+\frac{1}{r^{2} \sin \theta} \frac{\partial^{2} \Psi}{\partial \phi^{2}}+k^{2} \Psi=0 .
$$


This differential equation can be solved by a separation ansatz $\Psi(r, \theta, \phi)=R(r) \Theta(\theta) \Phi(\phi)$, which splits equation 2.44 into three separate differential equations for angular and radial components.

$$
\begin{aligned}
\frac{d^{2} \Phi}{d \phi^{2}}+m^{2} \Phi & =0 \\
\frac{1}{\sin \theta} \frac{d}{d \theta}\left(\sin \theta \frac{d \Theta}{d \theta}\right)+\left[n(n+1)-\frac{m^{2}}{\sin ^{2} \theta}\right] \Theta & =0 \\
\frac{d}{d r}\left(r^{2} \frac{d R}{d r}\right)+\left[k^{2} r^{2}-n(n-1)\right] R & =0 .
\end{aligned}
$$

The separation constants $m, n$ are determined by physical constraints, which will be discussed below. Well known function systems can be used to solve the separated differential equations $2.45-2.47$.

Equation 2.45 is solved by linear combinations of the sine and cosine functions

$$
\begin{aligned}
& \Phi_{e}=\cos (m \phi), \\
& \Phi_{o}=\sin (m \phi),
\end{aligned}
$$

where the subscripts denote even and odd functions. Their periodicity demands integer values for $m$.

The $\theta$-dependent differential equation 2.46 can be solved - in analogy to the also analytically solvable problem of the hydrogen atom - by the associated Legendre functions of the first kind, $P_{n}^{m}(\cos \theta)$ with $n=m, m+1, m+2 \ldots$ for the separation constants $m, n$. It is interesting to note, that in the case of hydrogen the separation constants will lead to the quantum numbers of bound orbitals. In contrast, $m$ and $n$ are interpreted as modes of the scattered light within the Mie formalism, which will be further discussed below.

Also the radial equation 2.47 can be brought into a well known form by introducing $\rho=k r$ and substituting $Z=R \sqrt{\rho}$,

$$
\rho \frac{d}{d \rho}\left(\rho \frac{d Z}{d \rho}\right)+\left[\rho^{2}-\left(n+\frac{1}{2}\right)^{2}\right] Z=0 .
$$

The linear independent solutions for this type of differential equation are the Bessel functions of first and second kind $J_{n}$ and $Y_{n}$. By re-substitution of $Z$ and $\rho$, the spherical Bessel functions arise

$$
\begin{aligned}
& j_{n}(\rho)=\sqrt{\frac{\pi}{2 \rho}} J_{n+1 / 2}(\rho) \\
& y_{n}(\rho)=\sqrt{\frac{\pi}{2 \rho}} Y_{n+1 / 2}(\rho) .
\end{aligned}
$$

In principle, also other types of Bessel functions could be used to solve equation 2.47. This is in particular important for computational purposes, where usually the type of Bessel function is chosen which shows the most convenient computation properties [36, 46]. For this reason, in the general solution of the generation function $\Psi$ found by the separation ansatz, $z_{n}(k r)$ denotes all possible types of Bessel functions:

$$
\begin{aligned}
& \Psi_{\text {emn }}=\cos (m \phi) P_{n}^{m}(\cos \theta) z_{n}(k r) \\
& \Psi_{\text {omn }}=\sin (m \phi) P_{n}^{m}(\cos \theta) z_{n}(k r),
\end{aligned}
$$


which is again subdivided into even and odd functions. This solution of the scalar wave function is still general and will be further restricted to physically appropriate function types and values of the separation constants. But already here, the vector harmonics $\vec{M}$ and $\vec{N}$ can be developed from the generation functions 2.51 with $\vec{c}=\vec{r}$.

$$
\begin{aligned}
\vec{M}_{e n m}= & -\frac{m}{\sin \theta} \sin (m \phi) P_{n}^{m}\left(\cos (\theta) z_{n}(\rho) \hat{\mathbf{e}}_{\theta}\right. \\
& -\cos (m \phi) \frac{d P_{n}^{m}(\cos \theta)}{d \theta} z_{n}(\rho) \hat{\mathbf{e}}_{\phi} \\
\vec{M}_{\text {onm }}= & \frac{m}{\sin \theta} \cos (m \phi) P_{n}^{m}\left(\cos (\theta) z_{n}(\rho) \hat{\mathbf{e}}_{\theta}\right. \\
& -\sin (m \phi) \frac{d P_{n}^{m}(\cos \theta)}{d \theta} z_{n}(\rho) \hat{\mathbf{e}}_{\phi} \\
\vec{N}_{\text {enm }}= & \frac{z_{n}(\rho)}{\rho} \cos (m \phi) n(n+1) P_{n}^{m}(\cos \theta) \hat{\mathbf{e}}_{r} \\
& +\cos (m \phi) \frac{d P_{n}^{m}(\cos \theta)}{d \theta} \frac{1}{\rho} \frac{d}{d \rho}\left[\rho z_{n}(\rho)\right] \hat{\mathbf{e}}_{\theta} \\
& -m \sin (m \phi) \frac{d P_{n}^{m}(\cos \theta)}{d \theta} \frac{1}{\rho} \frac{d}{d \rho}\left[\rho z_{n}(\rho)\right] \hat{\mathbf{e}}_{\phi} \\
\vec{N}_{\text {onm }}= & \frac{z_{n}(\rho)}{\rho} \sin (m \phi) n(n+1) P_{n}^{m}(\cos \theta) \hat{\mathbf{e}}_{r} \\
& +\sin (m \phi) \frac{d P_{n}^{m}(\cos \theta)}{d \theta} \frac{1}{\rho} \frac{d}{d \rho}\left[\rho z_{n}(\rho)\right] \hat{\mathbf{e}}_{\theta} \\
& +m \cos (m \phi) \frac{d P_{n}^{m}(\cos \theta)}{d \theta} \frac{1}{\rho} \frac{d}{d \rho}\left[\rho z_{n}(\rho)\right] \hat{\mathbf{e}}_{\phi} .
\end{aligned}
$$

The vector harmonics constitute an orthonormal basis set of functions which satisfies the spherical geometry. They span the solution space of the Maxwell equations, thus, arbitrary fields can be expanded to infinite series of the vector harmonics.

Expanding the fields in vector harmonics: In particular the incident, inner and scattered fields of a homogeneous sphere, as depicted in Fig. 2.3 can be expressed as an infinite series of the vector harmonics

$$
\vec{E}=\sum_{m=0}^{\infty} \sum_{n=m}^{\infty}\left(B_{e m n} \vec{M}_{e m n}+B_{o m n} \vec{M}_{o m n}+A_{e m n} \vec{N}_{e m n}+A_{o m n} \vec{N}_{o m n}\right)
$$

where the expansion coefficients for each mode (even/odd, $m, n$ ) can be derived by projecting the fields on the modes of the vector harmonics

$$
B_{e m n}=\frac{\int_{0}^{2 \pi} \int_{0}^{\pi} \vec{E} \cdot \vec{M}_{e m n} \sin \theta d \theta d \phi}{\int_{0}^{2 \pi} \int_{0}^{\pi}\left|\vec{M}_{e m n}\right|^{2} \sin \theta d \theta d \phi} .
$$


From this point on, all further steps of deriving the Mie formalism can be obtained by restraining the expansion coefficients on modes which are $\neq 0$ and subtypes of function systems which satisfy physical reasoning. For example, the fields within the sphere can only contain Bessel functions of the first kind, as the second kind will diverge at the origin. Also it can be shown, that $m$ equals 1 for all fields.

The expansion of the incident fields in an infinite series of vector harmonics can be found as

$$
\begin{aligned}
\vec{E}_{i n c} & =E_{0} \sum_{n=1}^{\infty} i^{n} \frac{2 n+1}{n(n+1)}\left(\vec{M}_{o 1 n}^{(j)}-i \vec{N}_{e 1 n}^{(j)}\right) \\
\vec{H}_{i n c} & =-\frac{k}{\omega \mu} E_{0} \sum_{n=1}^{\infty} i^{n} \frac{2 n+1}{n(n+1)}\left(\vec{M}_{e 1 n}^{(j)}+i \vec{N}_{o 1 n}^{(j)}\right) .
\end{aligned}
$$

The superscribed index ${ }^{(j)}$ denotes the restriction on the Bessel functions of the first kind, as indicated above. By substituting the incident fields in the boundary conditions 2.38, the fields inside the sphere can be obtained

$$
\begin{aligned}
& \vec{E}_{\text {sphere }}=E_{0} \sum_{n=1}^{\infty} i^{n} \frac{2 n+1}{n(n+1)}\left(c_{n} \vec{M}_{o 1 n}^{(j)}-i d_{n} \vec{N}_{e 1 n}^{(j)}\right) \\
& \vec{H}_{\text {sphere }}=-\frac{k_{\text {sphere }}}{\omega \mu_{\text {sphere }}} E_{0} \sum_{n=1}^{\infty} i^{n} \frac{2 n+1}{n(n+1)}\left(d_{n} \vec{M}_{e 1 n}^{(j)}+i c_{n} \vec{N}_{o 1 n}^{(j)}\right)
\end{aligned}
$$

The scattered fields can be expressed in the same manner:

$$
\begin{aligned}
\vec{E}_{\text {scat }} & =E_{0} \sum_{n=1}^{\infty} i^{n} \frac{2 n+1}{n(n+1)}\left(i a_{n} \vec{N}_{e 1 n}^{\left(h^{-}\right)}-b_{n} \vec{M}_{o 1 n}^{\left(h^{-}\right)}\right) \\
\vec{H}_{\text {scat }} & =\frac{k}{\omega \mu} E_{0} \sum_{n=1}^{\infty} i^{n} \frac{2 n+1}{n(n+1)}\left(i b_{n} \vec{N}_{o 1 n}^{\left(h^{-}\right)}+a_{n} \vec{M}_{e 1 n}^{\left(h^{-}\right)}\right) .
\end{aligned}
$$

Here, the superscribed index $(-h)$ indicates the use of another subtype of Bessel functions, so called Hankel functions, which can be interpreted as the far field approximation $k r \gg n^{2}$ of the spherical Bessel functions:

$$
\begin{aligned}
& h_{n}^{+}(k r)=\frac{(-i)^{n} e^{i k r}}{i k r} \\
& h_{n}^{-}(k r)=-\frac{i^{n} e^{-i k r}}{i k r} .
\end{aligned}
$$

The expansion coefficients $a_{n}$ and $b_{n}$, which are termed scattering coefficients, weight the contributions of the normal modes $M_{n}$ and $N_{n}$ of the sphere. By introducing the size parameter $x=k R$ with $k$ being the wave number and $R$ the radius of the sphere, and the relative 
refractive index $m_{\text {rel }}=n_{\text {sphere }} / n_{\text {out }}=n_{\text {sphere }}$ we obtain

$$
\begin{aligned}
a_{n}= & \frac{\mu_{\text {out }} n_{\text {sphere }}^{2} j_{n}\left(n_{\text {sphere }} x\right) \partial\left(x j_{n}(x)\right) / \partial x-}{\mu_{\text {out }} n_{\text {sphere }}^{2} j_{n}\left(n_{\text {sphere }} x\right) \partial\left(x h_{n}^{+}(x)\right) / \partial x-} \\
& \frac{-\mu_{\text {sphere }} j_{n}(x) \partial\left(n_{\text {sphere }} x j_{n}\left(n_{\text {sphere }} x\right)\right) / \partial\left(n_{\text {sphere }} x\right)}{-\mu_{\text {sphere }} h_{n}^{+}(x) \partial\left(n_{\text {sphere }} x j_{n}\left(n_{\text {sphere }} x\right)\right) / \partial\left(n_{\text {sphere }} x\right)} \\
b_{n}= & \frac{\mu_{\text {sphere }} j_{n}\left(n_{\text {sphere }} x\right) \partial\left(x j_{n}(x)\right) / \partial x-}{\mu_{\text {sphere }} j_{n}\left(n_{\text {sphere }} x\right) \partial\left(x h_{n}^{+}(x)\right) / \partial x-} \\
& -\mu_{\text {out }} j_{n}(x) \partial\left(n_{\text {sphere }} x j_{n}\left(n_{\text {sphere }} x\right)\right) / \partial\left(n_{\text {sphere }} x\right) \\
& -\mu_{\text {out }} h_{n}^{+}(x) \partial\left(n_{\text {sphere }} x j_{n}\left(n_{\text {sphere }} x\right)\right) / \partial\left(n_{\text {sphere }} x\right)
\end{aligned}
$$

With the scattering coefficients at hand, the matrix elements of the amplitude scattering matrix $S_{i}$ can be expressed explicitly. Due to the spherical symmetry of the problem the non-diagonal elements of the scattering matrix, $S_{3}$ and $S_{4}$ equal zero. This corresponds to the conservation of polarization state of the incident light in the scattering process. The derivation of the Mie formalism for scattering on homogeneous spherical particles can now be completed by presenting the expressions for the diagonal elements:

$$
\begin{aligned}
S_{1} & =\sum_{n} \frac{2 n+1}{n(n+1)}\left(a_{n} \frac{P_{n}^{1}}{\sin \theta}+b_{n} \frac{d P_{n}^{1}}{d \theta}\right) \\
S_{2} & =\sum_{n} \frac{2 n+1}{n(n+1)}\left(a_{n} \frac{d P_{n}^{1}}{d \theta}+b_{n} \frac{P_{n}^{1}}{\sin \theta}\right) .
\end{aligned}
$$

In the same manner the vector harmonics can be used to expand the fields in a coated sphere and thus calculate the appropriate scattering coefficients. For the case of a coated sphere, further boundary conditions have to be taken into account.

Boundary conditions for a coated sphere: The derivation of the formalism for coated spheres can be traced back to Aden and Kerker [47]. It is obtained in complete analogy to the solution for homogeneous spheres. The notations for fields and complex refractive indices are depicted in Fig. 2.5. Again, the fields outside the scatterer $\vec{E}_{\text {out }}, \vec{H}_{\text {out }}$ consist of the sum of incident and scattered fields

$$
\begin{aligned}
\vec{E}_{\text {out }} & =\vec{E}_{\text {inc }}+\vec{E}_{\text {scat }} \\
\vec{H}_{\text {out }} & =\vec{H}_{\text {inc }}+\vec{H}_{\text {scat }}
\end{aligned}
$$

In addition to the constraints valid for homogeneous scatterers, energy conservation at the boundary between core and shell has to be claimed. Therefore, the boundary conditions 2.38 are extended to

$$
\begin{array}{rrl}
\left(\vec{E}_{\text {shell }}-\vec{E}_{\text {core }}\right) \times \hat{r}=0 & \left(\vec{H}_{\text {shell }}-\vec{H}_{\text {core }}\right) \times \hat{r}=0 & r=R-d \\
\left(\vec{E}_{\text {out }}-\vec{E}_{\text {shell }}\right) \times \hat{r}=0 & \left(\vec{H}_{\text {out }}-\vec{H}_{\text {shell }}\right) \times \hat{r}=0 & r=R .
\end{array}
$$

Analog to the derivations above, the electromagnetic fields outlined in Fig. 2.5 are expanded in infinite series of vector harmonics. The result for the fields of the core $\left(\vec{E}_{\text {core }}, \vec{H}_{\text {core }}\right)$ is 


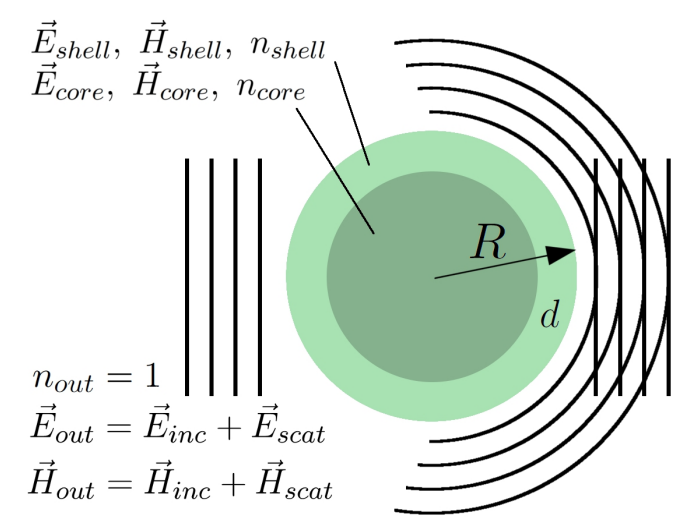

Figure 2.5: Geometry and fields of a core-shell system. A sphere with radius $R$ is surrounded by vacuum $(n=1)$. The sphere exhibits two homogeneous regions, a core with radius $R-d$ and complex refractive index $n_{\text {core }}$ and an outer shell with thickness $d$ and complex refractive index $n_{\text {shell }}$. The notations of the fields are chosen accordingly.

equivalent to the expression for $\left(\vec{E}_{\text {sphere }}, \vec{H}_{\text {sphere }}\right)$ given by equation 2.59 . Also the expressions for incident (Eq. 2.58) and scattered fields (Eq. 2.60) obtained above can be reused. In addition, the fields in the region of the shell have to be expanded in vector harmonics:

$$
\begin{aligned}
\vec{E}_{\text {shell }} & =E_{0} \sum_{n=1}^{\infty} i^{n} \frac{2 n+1}{n(n+1)}\left(f_{n} \vec{M}_{o 1 n}^{(j)}-i g_{n} \vec{N}_{e 1 n}^{(j)}+v_{n} \vec{M}_{o 1 n}^{(y)}-i w_{n} \vec{N}_{e 1 n}^{(y)}\right) \\
\vec{H}_{\text {shell }} & =-\frac{k_{\text {shell }}}{\omega \mu_{\text {shell }}} E_{0} \sum_{n=1}^{\infty} i^{n} \frac{2 n+1}{n(n+1)}\left(g_{n} \vec{M}_{e 1 n}^{(j)}+i f_{n} \vec{N}_{o 1 n}^{(j)}+w_{n} \vec{M}_{o 1 n}^{(y)}+i v_{n} \vec{N}_{e 1 n}^{(y)}\right) .
\end{aligned}
$$

In this case the spherical Bessel functions can neither be reduced to one kind as in the core, because both first and second kind are finite within $R-d<r<R$, or approximated by far field as the scattered fields. Therefore, four more expansion coefficients $f_{n}, g_{n}, v_{n}, w_{n}$ arise from equation 2.65 .

By substituting the fields of core, shell and outside the scatterer expanded in vector harmonics into the respective boundary conditions 2.64 , an equation system for the expansion coefficients arises. In particular the scattering coefficients $a_{n}, b_{n}$ can be obtained. To simplify the description, the radial components are represented by the so-called Ricati-Bessel functions $\psi, \xi$ and $\chi$ :

$$
\psi_{n}(\rho)=\rho j_{n}(\rho) \quad \xi_{n}(\rho)=\rho h_{n}^{(+)}(\rho) \quad \chi_{n}(\rho)=-\rho y_{n}(\rho) .
$$

Further, $\mu_{\text {core }}=\mu_{\text {shell }}=\mu_{\text {out }}$ is assumed and the size parameters $x=k(R-d)$ and $y=k R$ are introduced. Then the scattering coefficients for a coated sphere read 


$$
\begin{aligned}
a_{n}= & \frac{\psi_{n}(y)\left[\partial \psi_{n}\left(n_{\text {shell }} y\right) / \partial\left(n_{\text {shell }} y\right)-A_{n} \partial \chi_{n}\left(n_{\text {shell }} y\right) / \partial\left(n_{\text {shell }} y\right)\right]-}{\xi_{n}(y)\left[\partial \psi_{n}\left(n_{\text {shell }} y\right) / \partial\left(n_{\text {shell }} y\right)-A_{n} \partial \chi_{n}\left(n_{\text {shell }} y\right) / \partial\left(n_{\text {shell }} y\right)\right]-} \\
& \frac{-n_{\text {shell }}\left(\partial \psi_{n}(y) / \partial y\right)\left[\psi_{n}\left(n_{\text {shell }} y\right)-A_{n} \chi_{n}\left(n_{\text {shell }} y\right)\right]}{-n_{\text {shell }}\left(\partial \xi_{n}(y) / \partial y\right)\left[\psi_{n}\left(n_{\text {shell }} y\right)-A_{n} \chi_{n}\left(n_{\text {shell }} y\right)\right]} \\
b_{n}= & \frac{n_{\text {shell }} \psi_{n}(y)\left[\partial \psi_{n}\left(n_{\text {shell }} y\right) / \partial\left(n_{\text {shell }} y\right)-B_{n} \partial \chi_{n}\left(n_{\text {shell }} y\right) / \partial\left(n_{\text {shell }} y\right)\right]-}{n_{\text {shell }} \xi_{n}(y)\left[\partial \psi_{n}\left(n_{\text {shell }} y\right) / \partial\left(n_{\text {shell }} y\right)-B_{n} \partial \chi_{n}\left(n_{\text {shell }} y\right) / \partial\left(n_{\text {shell }} y\right)\right]-} \\
& \frac{-\partial \psi_{n}(y) / \partial y\left[\psi_{n}\left(n_{\text {shell }} y\right)-B_{n} \chi_{n}\left(n_{\text {shell }} y\right)\right]}{} \\
& -\partial \xi_{n}(y) / \partial y\left[\psi_{n}\left(n_{\text {shell }} y\right)-B_{n} \chi_{n}\left(n_{\text {shell }} y\right)\right]
\end{aligned}
$$

with

$$
\begin{aligned}
A_{n}= & \frac{n_{\text {shell }} \psi_{n}\left(n_{\text {shell }} x\right) \partial \psi_{n}\left(n_{\text {core }} x\right) / \partial\left(n_{\text {core }} x\right)-}{n_{\text {shell }} \chi_{n}\left(n_{\text {shell }} x\right) \partial \psi_{n}\left(n_{\text {core }} x\right) / \partial\left(n_{\text {core }} x\right)-} \\
& \frac{-n_{\text {core }} \partial \psi_{n}\left(n_{\text {shell }} x\right) / \partial\left(n_{\text {shell }} x\right) \psi_{n}\left(n_{\text {core }} x\right)}{-n_{\text {core }} \partial \chi_{n}\left(n_{\text {shell }} x\right) / \partial\left(n_{\text {shell }} x\right) \psi_{n}\left(n_{\text {core }} x\right)} \\
B_{n}= & \frac{n_{\text {shell }} \psi_{n}\left(n_{\text {core }} x\right) \partial \psi_{n}\left(n_{\text {shell }} x\right) / \partial\left(n_{\text {shell }} x\right)-}{n_{\text {shell }} \chi_{n}\left(n_{\text {shell }} x\right) \partial \psi_{n}\left(n_{\text {core }} x\right) / \partial\left(n_{\text {core }} x\right)-} \\
& \frac{-n_{\text {core }} \psi_{n}\left(n_{\text {shell }} x\right) \partial \psi_{n}\left(n_{\text {core }} x\right) / \partial\left(n_{\text {core }} x\right)}{-n_{\text {core }} \partial \psi_{n}\left(n_{\text {core }} x\right) / \partial\left(n_{\text {core }} x\right) \chi_{n}\left(n_{\text {shell }} x\right)} .
\end{aligned}
$$

Though these expressions are of a quite complicated form, they consist solely of well known and tabulated function systems and can therefore used for computing the scattered field of a coated sphere. This extension of the Mie-formalism is able to describe light scattering from systems with an outer shell with clearly different optical properties compared to the inner part of the particles. Algorithms for coated spheres have been used for example to study the response from nucleated biological cells [48]. Since the first derivation of this formalism by Kerker and Aden [47], several computing algorithms have been proposed $([36,46]$ and references therein). They differ mainly in the choice of recursively defined types of Bessel functions, which can in some cases produce large errors for increasing size parameters. For simulating scattering patterns of core-shell systems in chapter 4.5.3, an algorithm developed by Shen $[49,46]$ was used.

In order to visualize the physical content of the rather complicated mathematical expressions, obtained for homogeneous and coated spherical particles, in the next section the structure of the normal modes will be further analyzed. Also the dependency of scattering cross-sections of spherical particles from the obtained scattering coefficients $2.61,2.62$ will be considered and the explicit scattered intensity $I(r, \theta, \phi)$ of homogeneous and coated spheres will be discussed.

Visualization of the solutions: In order to translate the mathematical relationships obtained in the last paragraphs into observation quantities and to gain a more intuitive understanding, the normal modes can be visualized. In Fig. 2.6 a, an exception from the original work of Gustav Mie on gold particles in a solution [43] is presented. The transversal components, i.e. the lines of electric flux on a virtual sphere around the scattering particle are displayed for the lowest four normal modes $N_{n}$ and $M_{n}$. Their structure illustrates that the 


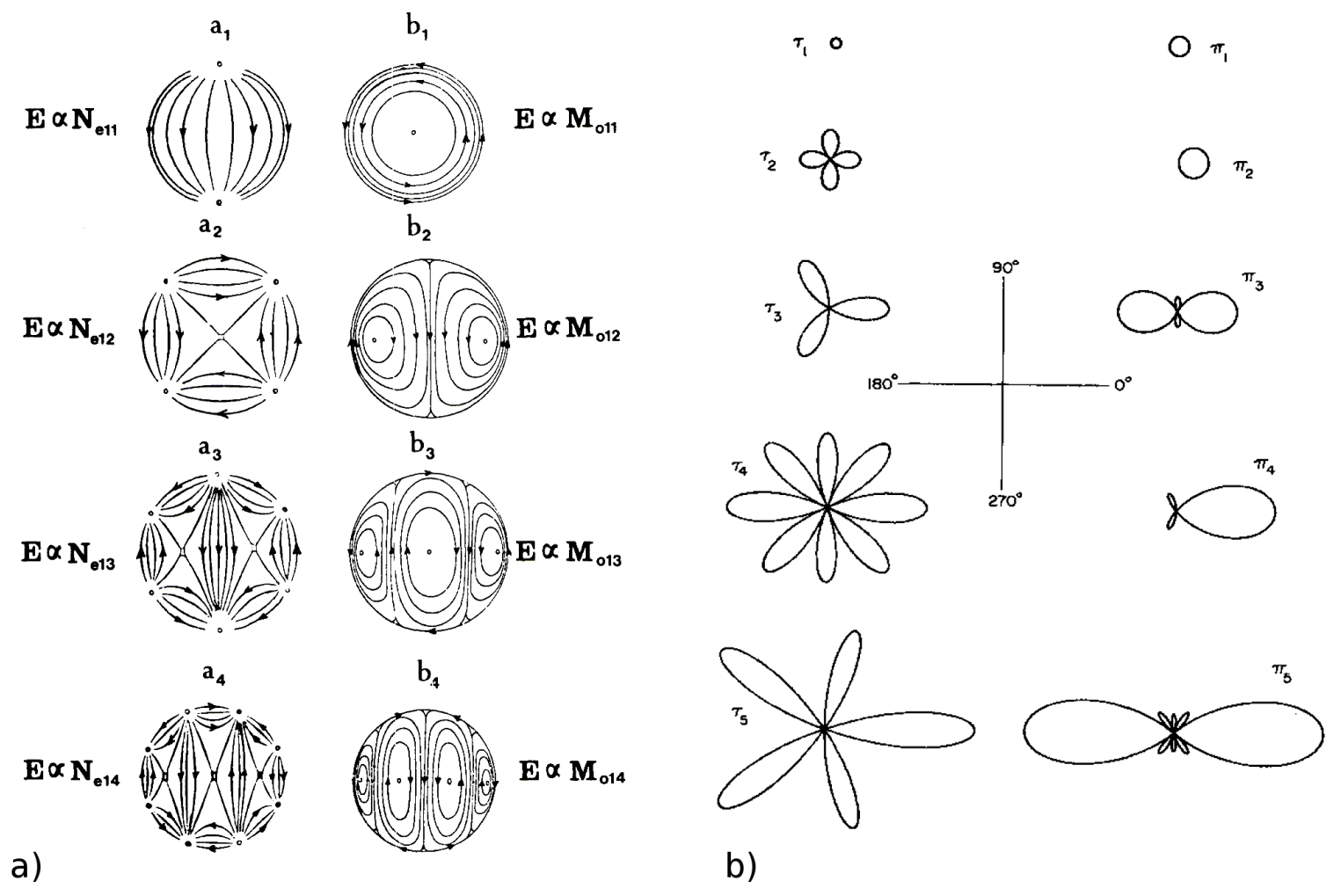

Figure 2.6: a) Transversal components of the normal modes $N_{n}$ and $M_{n}$ for $n=1$ to 4 . The field lines on a spherical surface around the scatterer are displayed, sources and sinks indicate points where the transversal component vanishes and only radial components are apparent. From [43]. b) $\Theta$-components of the normal modes for $n=1$ to 5 . Higher modes are needed to describe the scattering pattern of larger particles. Therefore, the more pronounced forward direction of the scattered light for larger particles can be understood as asymmetry of the higher $\Theta$-components. From [36]

series expansion in normal modes is the electrodynamic analogon to the well-known multipole expansion from electrostatics.

For small scatterers compared to the wavelength of the light and thus values of the size parameter $x \ll 1$, higher orders of the series expansion can be neglected. The first mode $n=1$ yields again the well-known case of Rayleigh scattering with pure dipole characteristics. For increasing $x$-values, also higher orders of the series expansion have to be accounted for. Their influence on the characteristics of the angular distribution can be traced in the $\Theta$ component of the normal modes. Therefore, the angular functions $\pi_{n}$ and $\tau_{n}$ are introduced with

$$
\pi_{n}=\frac{P_{n}^{1}}{\sin \theta} \quad \tau_{n}=\frac{\partial P_{n}^{1}}{\partial \theta}
$$

The initial five orders of the $\Theta$-components are depicted in Fig. 2.6 b. From the third mode on, they exhibit an asymmetry with preference on the forward direction. This observation corresponds to the relationship between the size parameter $x=R / \lambda$ and the scattering characteristics, in particular that larger particles scatter more prominently in forward direction. 


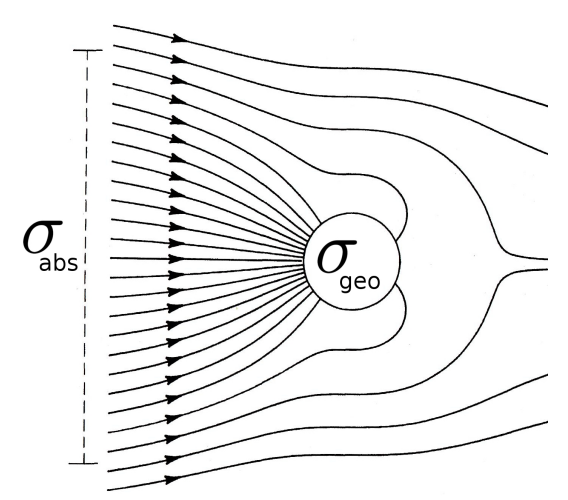

Figure 2.7: The field lines of the effective pointing vector demonstrate the extinction paradox: For excitation energies matching atomic resonances, the particle interacts strongly with the electromagnetic field and appears to be bigger. From [36].

Scattering and extinction cross-sections: In order to quantify the ability of a spherical object to scatter light, again the scattering cross-section can be calculated,

$$
\sigma_{\text {sphere }}=\frac{2 \pi}{k^{2}} \sum_{n=1}^{\infty}(2 n+1)\left(\left|a_{n}\right|^{2}+\left|b_{n}\right|^{2}\right) .
$$

Also a cross-section for the total extinction, i.e. the sum of scattered and absorbed intensity per incident intensity, can be expressed by the scattering coefficients

$$
\sigma_{\text {ext }}=\frac{2 \pi}{k^{2}} \sum_{n=1}^{\infty}(2 n+1) \operatorname{Re}\left\{a_{n}+b_{n}\right\} .
$$

Far away from electronic resonances, where the deviation of $n_{\text {sphere }}$ from unity is small, the extinction cross-section might be considerably smaller than the geometrical cross-section $\pi R^{2}$, however, the limit of the extinction cross-section for large size parameters $x \rightarrow \infty$ equals twice the geometric cross-section. This relation, which appears to be contra-intuitive at first sight is called the extinction paradox, which is illustrated by Fig. 2.1.3. It can be understood by considering the limit of the absorption cross-section, which will approach the geometric cross-section. In addition, the scattered field of a circular disk and a circular aperture have to be equal according to Babinet's Theorem [38]. Therefore, the same amount of light as absorbed will also be scattered, which in total explains the factor of two.

Close to atomic resonances the absorption cross-section alone might become larger than the geometrical cross-section. Fig. 2.1.3 depicts the field lines of the effective Pointing vector in the presence of a highly absorbing sphere. The fields are disturbed to a much larger extend than just the geometrical size, therefore a highly absorbing particle appears to be bigger.

Scattering patterns calculated with Mie's theory: For connecting the theoretical results with the experimentally obtained scattering patterns, the scattered intensity from a homogeneous spherical particle and analog from a coated sphere can be further examined. The transformation for incident to scattered fields was given by equation 2.40. By recalling that the non-diagonal elements were found to equal zero, and assuming linearly polarized light (per definition in perpendicular direction), equation 2.40 can be reduced to 


$$
\left(\begin{array}{c}
E_{\| s c a t} \\
E_{\perp s c a t}
\end{array}\right)=\frac{e^{i k(r-z)}}{-i k r}\left(\begin{array}{cc}
S_{2} & 0 \\
0 & S_{1}
\end{array}\right)\left(\begin{array}{c}
0 \\
E_{\perp i n c}
\end{array}\right),
$$

yielding the individual components

$$
\begin{aligned}
\vec{E}_{\perp s c a t} & =\frac{e^{i k(r-z)}}{-i k r} S_{1} E_{\perp i n c}, \\
\vec{E}_{\| s c a t} & =0 .
\end{aligned}
$$

Therefore, the scattered light will be also linearly polarized perpendicular to the scattering plane. The scattered intensity, which denotes the observable measure of energy flux per time and area unit, can be calculated as the square of the scattered field amplitude

$$
I_{\text {scat }}(r, \theta, \phi)=\frac{k}{2 \omega \mu}\left|\vec{E}_{\text {scat }}\right|^{2}=\frac{k}{2 \omega \mu} \frac{1}{k^{2} r^{2}}\left|S_{1}\right|^{2} E_{\perp i n c}^{2} .
$$

By substituting the incoming intensity $I_{i n c}=k /(2 \omega \mu) E_{\perp i n c}^{2}$ we obtain

$$
I_{\text {scat }}=\frac{1}{k^{2} r^{2}}\left|S_{1}\right|^{2} I_{i n c}
$$

Therefore, the computation of scattering patterns can be achieved by calculating $S_{1}$ and $S_{2}$ with the input parameters $x=k R$ and $m_{\text {rel }}=n_{\text {sphere }} / n_{\text {out }}$ in the case of a homogeneous sphere and the respective size parameters $x=k(R-d)$ and $y=k R$ and refractive indices of core and shell. The number of modes which have to be taken into account increases with increasing size parameters of the scatterers. Termination conditions stop the algorithms which are used for computation, when the contribution of further modes will be small. These conditions are necessary because with decreasing contribution of the modes, the numerical errors from the calculations of the implemented Bessel function will grow and might even lead to a divergence of the series expansion.

The scattered intensity in perpendicular and parallel direction from a homogeneous sphere with size parameter of $x=50$ and a refractive index of $n_{\text {sphere }}=1.007+i \cdot 0.04$ is shown in Fig. 2.8 a. The scattered light as a function of scattering angle reveals the typical lobes of Mie-scattering which correspond to concentrical rings with nearly equidistant minima in the scattering patterns. This characteristic pattern also points to the close connection between scattering from a sphere and the Airy pattern of a circular aperture. One fundamental principle of diffraction theory is that the scattering pattern of an object can be calculated by the Fourier transform of its spatial function, for example the charge density distribution of a molecule or a simple cylindrical function of a circular aperture $[42,50]$. The ring structures of the scattering patterns calculated with Mie's theory show that this principle is also implicitly contained in the complicated formalism derived above. Beyond this, the Mie-simulations are able to also account for the material properties of the scatterer and the polarization of the incident and scattered light.

The influence of the polarization of the incident light can be seen in the difference between the red and the green curve in Fig. 2.8 a. The scattered light in polarization direction of the incident wave, i.e. the green curve, exhibits a global minimum close to $90^{\circ}$. This minimum corresponds to the limit of Rayleigh scattering where no radiation is emitted in the polarization direction of the incoming light.

The width of the lobes will become smaller with increasing size of the sphere. This can be again related to the scattering pattern being the Fourier transform of the scatterer, thus 

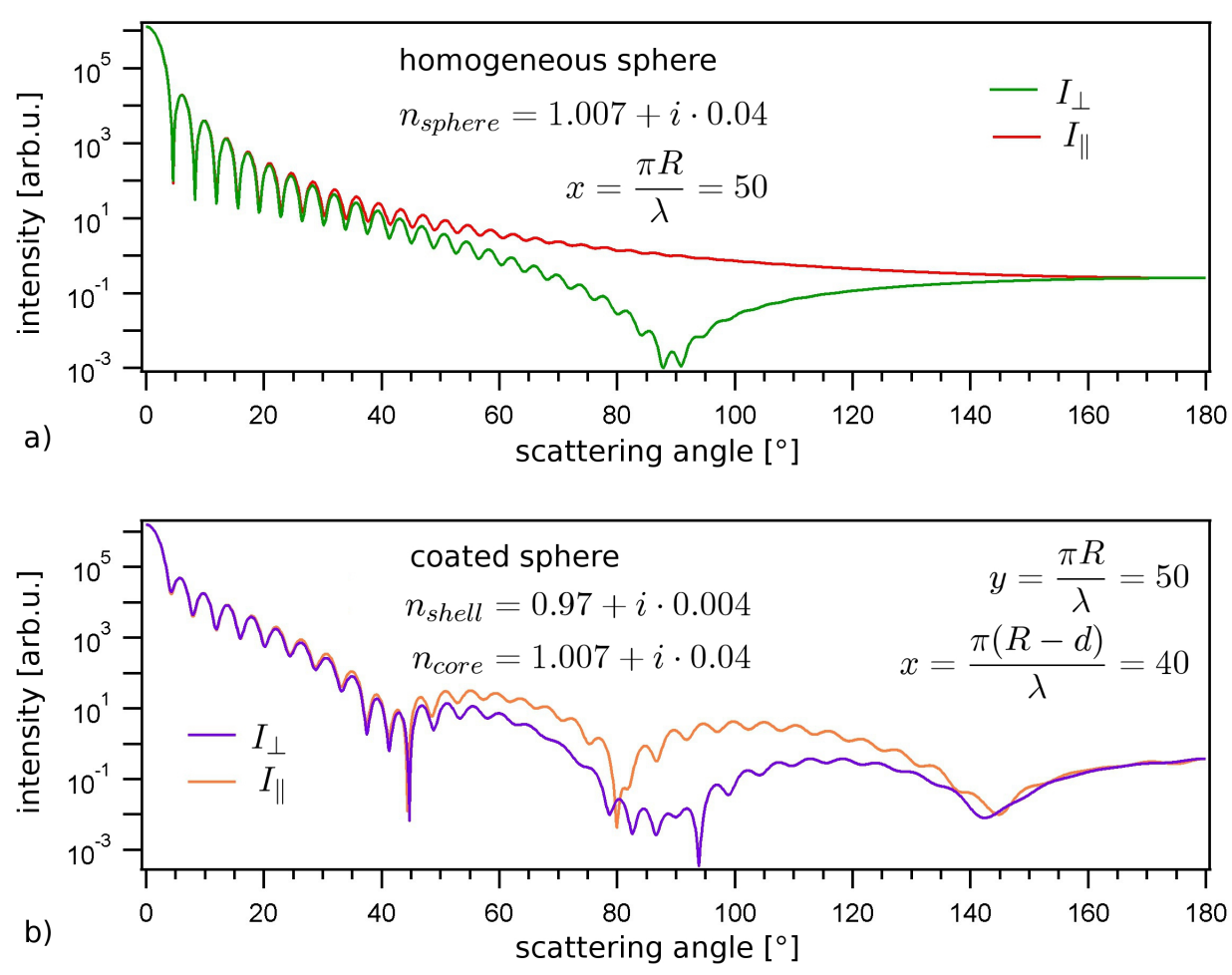

Figure 2.8: Calculated profiles of a) homogeneous and b) coated spheres within the Mie formalism. The minimum close to $90^{\circ}$ scattering angle observed for the perpendicular intensity profiles corresponds to the Rayleigh limit for small spheres. The high-frequency oscillation in a) and b) correspond to the overall size of the particles, the low-frequency modulation in b) indicates a second characteristic length scale in the particle, the thickness of the outer shell.

structures observed in the frequency domain of the scattering pattern correspond to a characteristic length scale of the scatterer. The oscillations in Fig. 2.8 a correspond to the only characteristic length of a homogeneous sphere, its overall size.

In contrast, the scattering profiles of a core-shell system, which are displayed in Fig. $2.8 \mathrm{~b}$ (again for parallel and perpendicular direction) indicate two characteristic length scales in the particle, as the fine lobes are superimposed by a low frequency oscillation. This low frequency can be related to the thickness of the outer shell which constitutes the second characteristic length in this particle.

Another approach to understand the modulation observed in the profiles of Fig. $2.8 \mathrm{~b}$ is their interpretation as a beating pattern between the slightly different widths of the lobes in the scattered light produced by the inner and outer interface of the coated sphere.

A detailed discussion of changes in the scattering profiles based on a systematic study of variations of different parameters, such as the thickness of the shell and the optical constants, will be given in section 4.5.3.

Up to this point, the discussion of the interaction between light and clusters has been restricted to linear effects and static cases. In the next section, the assumption of linear processes of matter in the highly intense XUV pulses present in the current experiment will be discussed critically. 


\subsection{Atoms in intense XUV pulses}

Intense light fields are able to leave atoms highly excited and ionized. The degree of ionization and the underlying processes are dependent on the power density of the light, the wavelength, and also on the atomic species. This section gives a survey on the phenomena arising from the use of intense XUV light. The discussion concentrates on effects in single atoms, while the influence of the cluster surrounding will be discussed later in section 2.3.

Concepts are introduced to describe processes and regimes of the interaction between atoms and intense light fields of different photon energy. As a result of these considerations, the $90 \mathrm{eV}$ pulses with intensities of up to $5 \cdot 10^{14} \mathrm{~W} / \mathrm{cm}^{2}$ used in the experiments of this thesis can be assigned to a perturbative, photon dominated regime.

In this regime and in particular close to atomic resonances, the electronic structure of the studied target material is important. Experiments on atomic xenon gas at $90 \mathrm{eV}$ photon energy revealed a high degree of ionization [51]. The reason therefore can be found in the peculiar characteristics of xenon in the XUV range. Already neutral atoms exhibit high crosssections but atomic ions reveal even more extreme resonances. The absorption properties and the origin of the special energy level structure of xenon will be discussed.

\subsubsection{Wavelength dependent nonlinear processes}

The advent of short wavelength free-electron laser made high intensities from XUV to X rays accessible for the first time. Other light sources used to study light-matter interaction in the high energy range, such as synchrotron or high harmonic generation sources can only access processes, which are linear in intensity and can be described perturbatively.

Perturbative description: The energies of an atomic system are described quantummechanically (cf. [52], p. 480ff) by the Hamilton operator. For the unperturbed system, i.e. no field, the Hamiltonian is given by

$$
H_{0}=\underbrace{\frac{\mathbf{P}^{2}}{2 m}}_{E_{k i n}}+\underbrace{V(r)}_{E_{p o t}} .
$$

By solving Schrödinger's equation $E|\Psi\rangle=H_{0}|\Psi\rangle$ the orthonormal set of eigen states $\left|\psi_{n}\right\rangle$ with eigen energies $\epsilon_{n}$ can be found, which correspond to the orbitals of the atom. An incident lightwave induces a periodic perturbation of the system. The new Hamiltonian $H$ has eigen states different to $\left|\psi_{n}\right\rangle$, however for small perturbations, it can be expanded in the eigen system. Then $H$ reads

$$
H=H_{0}+W(t)=H_{0}+\lambda W_{1}+\lambda^{2} W_{2}+\ldots=H_{0}+W_{E D}+W_{M D}+W_{E Q} \cdots
$$

where $W_{E D}$ denotes the electric dipole operator, $W_{M D}$ the magnetic dipole operator, and $W_{E Q}$ the electric quadruple operator. The factor $\lambda \ll 1$ indicates, that the perturbation operators are sorted for their size. Thus, for small perturbations, i.e. weak oscillating fields, the dipole operator dominates the response and operators of higher orders can be neglected. From the projection of the dipole operator on two eigen states $\left|\psi_{i}\right\rangle,\left|\psi_{k}\right\rangle$ one obtains the transition probability $P_{i k}$ between those states due to the absorption of a single photon.

$$
\left\langle\psi_{i}\left|W_{E D}\right| \psi_{k}\right\rangle=P_{i k}
$$


Higher orders become more important for stronger light fields, where the probabilities for absorbing two or more photons increase.

In order to decide, whether a light field has to be considered strong, a first indicate can be given by the comparison of the light field strength to the atomic field strength unit [34]

$$
E_{a t}=\frac{1}{4 \pi \varepsilon_{0} a_{0}^{2}} \approx 5 \cdot 10^{9} \mathrm{~V} / \mathrm{cm}
$$

This field strength corresponds to an intensity of $4 \cdot 10^{16} \mathrm{~W} / \mathrm{cm}^{2}$. Already at intensities below this value, the perturbation from the light field can no longer be assumed small. Then the expansion of the Hamiltonian in the eigensystem of the unperturbed atom is no longer a good approximation, which is referred to as the perturbative breakdown. It is important to note, that this estimate neglects the photon energy of the respective light-field. As the discussion below will show, the light frequency plays a very important role for the ionization processes and the assignment to photon- or field-dominated regimes.

High intensity phenomena at different photon energies: In the long wavelength range (visible/IR) intensities up to $10^{20} \mathrm{~W} / \mathrm{cm}^{2}$ could be achieved in experiments. At these intensities various surprising effects were discovered (see for example [34], chapter 7 and references therein). Among them are above barrier ionization, high-harmonic generation, and so called resonant AC stark enhancement.

With the availability of FLASH and other short wavelength free-electron lasers (cf. section 3.1 ), access is provided to also study nonlinear processes in the high energy photon range. Up to now, only few experiments have been published in the VUV and XUV range [51, 53, 54] and in the X-ray regime $[55,56,57,58]$.

The processes which lead to the removal of electrons from the atomic union and in particular the onset of ionization are strongly wavelength dependent. Mainly two points have to be considered:

- How many photons are necessary to overcome the ionization potential?

- How long does the electron need to leave the atom in respect to the period of the lightwave?

The dominant process at the onset of ionization for three different wavelength regimes is displayed in Fig. 2.9 (curtesy by C. Bostedt). In Fig. 2.9 a the ionization process in the infrared range is illustrated. With photon energies of $1 \mathrm{eV}$ and less, single photo-ionization processes are impossible. If the field strength becomes high enough to bend the atomic potential sufficiently, bound electrons can tunnel out. For even stronger fields the electrons can leave directly over the barrier. In these processes many photons are absorbed by one electron, therefore this effect occurs only beyond the perturbative breakdown [34].

Towards higher photon energies (Fig. $2.9 \mathrm{~b}$ ) the onset of ionization is located in the perturbation limit. The uppermost valence shells might be already accessible in the UV or VUV range, depending on the target atom. Only few photons suffice to ionize further valence electrons.

In Fig. $2.9 \mathrm{c}$ the main ionization process in the high energy range is depicted. Inner shell electrons can be ionized by single photons starting from the XUV and soft X-ray regime. Even innermost shells are addressed in the case of hard X-rays. Due to the production of innershell vacancies, the photo-ionization processes are followed by subsequent decay processes. 


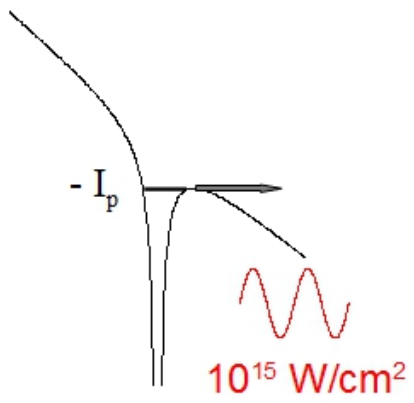

a) $\mathrm{E}_{\text {photon }} \sim 1 \mathrm{eV}$

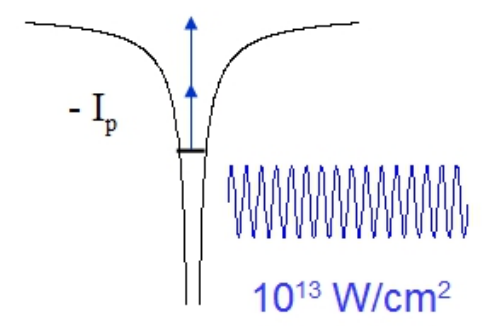

b) $\mathrm{E}_{\text {photon }} \sim 10 \mathrm{eV}$

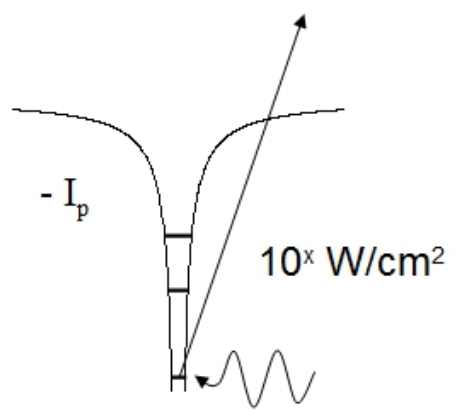

c) $\mathrm{E}_{\text {photon }} \geqq 100 \mathrm{eV}$

Figure 2.9: Photon energy dependent dominant process for the onset of ionization, visualizations are a curtesy by C. Bostedt. a) At small photon energies $(<1 \mathrm{eV})$, many photons are needed to ionize an atom. Only above a certain intensity threshold, the atomic potential is sufficiently bent to allow for tunneling of bound electrons. For much higher intensities above barrier ionization occurs. b) In the optical and ultraviolet spectral regime $(\approx 10 \mathrm{eV})$, only single or few photons are needed to ionize an atom. Therefore the onset of ionization is observed already at much smaller power densities and the ionization can be described perturbatively. c) In the X-ray spectral regime, inner shells are addressed by the incident photons. Photoionization processes are followed by subsequent decay of the inner-shell vacancy, leading to secondary (for example Auger) electron emission. Therefore already low intensity $\mathrm{X}$-ray beams (indicated as $10^{x} \mathrm{~W} / \mathrm{cm}^{2}$ ) can produce highly ionized atoms.

Non-radiative decays, namely Auger and Coster-Kronig transitions (electrons from a higher or the same shell fill the vacancy and transfer the energy difference to another electron which is liberated from the atomic union) lead to the emission of further electrons. Therefore, hard $\mathrm{X}$-ray radiation can produce highly ionized atoms already in the single photon limit.

Nevertheless, also for high energy radiation increasing intensities will eventually yield in the break-down of the perturbative description and a field-dominated response of the atoms. However, tunneling ionization will occur only at much higher field strengths as the high energy light field only addresses inner-shell electrons. In terms of time scales, the high frequency field will not allow for electrons to tunnel through the barrier during a singly half-cycle of the wave.

A measure of the ability of a field to couple directly to the atomic potential can be given by the cycle-averaged kinetic energy of a free electron in the wave $U_{p}$, referred to as ponderomotive potential

$$
U_{p}=\frac{I e^{2}}{2 \varepsilon_{0} c m_{e} \omega^{2}}
$$

where $I$ denotes the intensity of the light field and $\omega$ the frequency of the light. The ponderomotive potential yields a shift of the atomic energies respectively to the continuum, thus the resulting ionization potential is given by $I_{p}+U_{p}$ [34]. This effect is referred to as AC-Stark shift, which is able to produce intensity-induced resonances.

A comparison of the ionization potential to the ponderomotive potential can be used to estimate the probability for tunneling. The Keldysh parameter $\gamma$, which describes the transition 


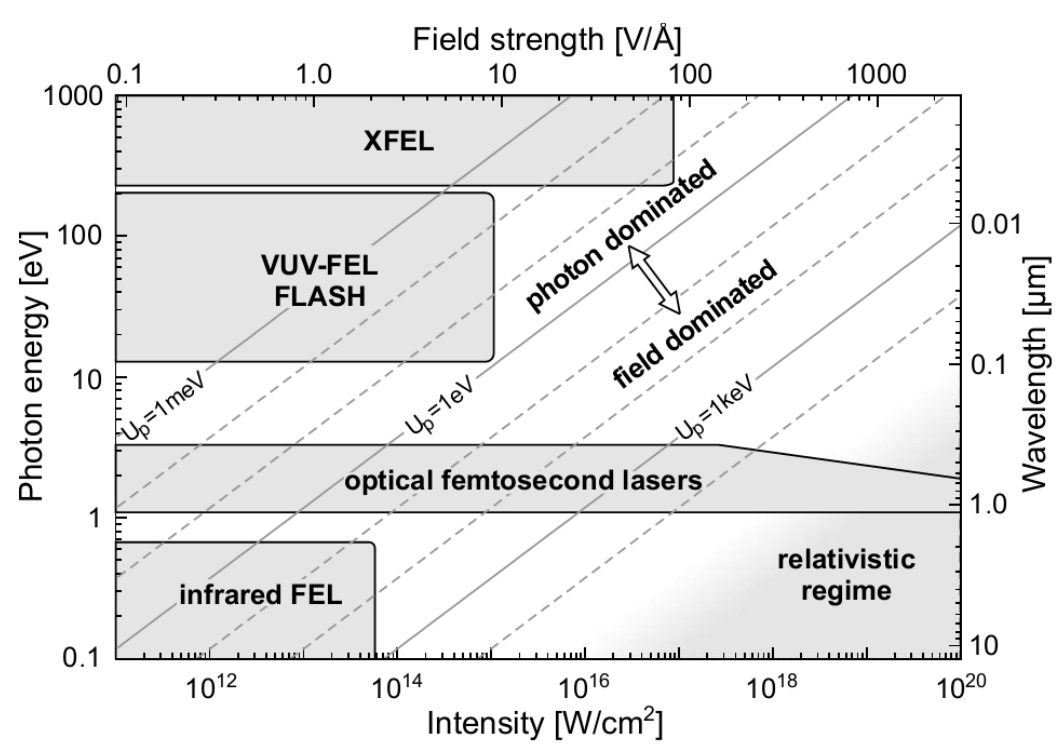

Figure 2.10: Photon energy and intensity dependent regimes, from [59]. The photon energy as a function of intensity for constant values of the ponderomotive potential yields lines, which also indicate the transitions between regimes (relativistic regime $U_{p}>10 \mathrm{keV}$, field dominated regime $U_{p} \geq 10 \mathrm{eV}$, photon dominated regime $U_{p} \leq 1 \mathrm{eV}$ ). Accessible energy/intensity values of different light sources are indicated by gray-shaded blocks.

between single- or multi-photon regime $(\gamma \gg 1)$ and tunneling regime $(\gamma \leq 1)$, is defined as

$$
\gamma=\sqrt{\frac{I_{P}}{2 U_{p}}}=\tau_{\text {tunnel }} \cdot \omega_{\text {laser }}
$$

This relationship also reflects the connection between the tunneling probability and the available time for an electron to tunnel the barrier, which is given by the laser period [59].

Constant values of the ponderomotive potential $U_{p}$ are displayed in Fig. 2.10 in a plot of photon energy per intensity [59]. Between 1 and $10 \mathrm{eV}$ the transition from photon dominated to field dominated regimes occurs, for $U_{p}$-values above $10 \mathrm{keV}$ even relativistic processes have to be considered. The shaded blocks indicate the attainable regimes of different light sources.

In the case of $90 \mathrm{eV}$ photon energy, the field dominated regime will not be reached with intensities below $10^{18} \mathrm{~W} / \mathrm{cm}^{2}$. In the experiments at the FLASH free-electron laser a focal power density of approximately $5 \cdot 10^{14} \mathrm{~W} / \mathrm{cm}^{2}$ was achieved (cf. section 3.2 .4 ). This results in a ponderomotive potential of approximately $10 \mathrm{meV}$. Therefore, the experiment discussed in this thesis is safely located in the perturbative regime. Even though also multi-photon absorption processes might occur [51], absorption of single photons and elastic scattering can be considered the predominant processes [34].

Far away from a field dominated regime the particular energy structure of the investigated material determines the response of atoms to the incident light. The peculiar electronic properties of xenon in the XUV range are discussed in the subsequent paragraph. 


\subsubsection{Ionization properties of atomic xenon in the XUV range}

Especially at $90 \mathrm{eV}$ photon energy, xenon constitutes an outstanding target material. The absorption cross section of neutral xenon atoms is for instance a factor of 34 higher compared to krypton [60], while only having 1.5 times more bound electrons. Thus, the topic of this section will be to examine the absorption characteristics of atomic xenon and its ions in the XUV range.

The measurement of absolute cross-sections is experimentally challenging [61]. Intense Xray and ion beams have to be merged over a long distance to enhance the signal-to-noise ratio, and all parameters are measured absolutely, the number of photons and ions, and the overlap integral between both. Due to available resonances with high cross-sections, early experiments using radiation from bending magnets were in fact carried out on xenon in the XUV range [62]. In the meanwhile, absolute cross sections for xenon in the XUV range are available up to $\mathrm{Xe}^{7+}[62,63,64,65,66,67]$. Atomic and ionic absorption cross sections are displayed in Fig. 2.11.

Resonant absorption via $4 d-n f, \epsilon f$ transitions: The photo absorption of xenon in the XUV range addresses mainly the $4 d$ shell. The typical shape of absorption edges of $s$-type shells, which is known for example from hard X-ray absorption spectra consists in a steep rise when the threshold energy is exceeded followed by an exponential decay for higher excitation energies. In contrast, the absorption cross-section from the $4 d$ shell exhibits a delayed onset with a broad maximum about $25 \mathrm{eV}$ above the ionization threshold [68]. This $4 d$ feature, referred to as giant resonance, can be observed for xenon (Fig. 2.11a) and adjacent elements in the periodic table from $\mathrm{Pd}$ (-8 nuclear charges) to Cs (+1 nuclear charge) [66]. It is connected to a two-well structure of the effective potential of the $f$-type vacuum levels, resulting from the competition of the centrifugal repulsion due to their high angular momentum and the Coulombic attraction from the core [69]. The probability density of the $n f$-states $(\mathrm{n}=4,5,6)$ in the inner well, close to the core, is low and therefore their overlap with the $4 d$ states is small. But for higher energies the $(\epsilon) f$-orbitals can gradually surmount the potential barrier, resulting in a higher overlap and an increasing ionization cross-section with a broad, resonance-like structure [70].

For elements in the periodic table with a number of nuclear charges $>56$, the $4 f$ orbitals turn into occupied levels in the ground state. In this transition from a more Rydberg-like vacuum level to a valence-type bound state, the orbitals drastically shift their density closer to the core [69], referred to as $4 f$-collapse [68].

Similar changes occur in the iso-nuclear series of xenon, in other words for a decreasing number of bound electrons at a constant number of nuclear charges. With rising charge state $q$, all orbitals are pulled towards the core and in particular the $4 f$ wave function collapses and gains a high overlap with the $4 d$-shell. This leads to a transition in the case of higher xenon ions from the direct photo-ionization process in the smooth giant resonance $4 d-\epsilon f$ with its maximum close to $90 \mathrm{eV}$ photon energy, to sharp, resonant transitions in excited, auto-ionizing states $4 d-n f$ mit $\mathrm{n}=4,5,6$, shifting towards higher photon energy with rising $q$. While up to $X e^{3+}$, most of the integral oscillator strength of the $4 d-n f, \epsilon f$ transition of about 10 (there are 10 electrons in the $4 \mathrm{~d}$ shell) contributes to the giant resonance [66], for higher charge states the ratio going into the $4 d-4 f$ resonances increases, also due to the decreasing number of possible excitation channels. 


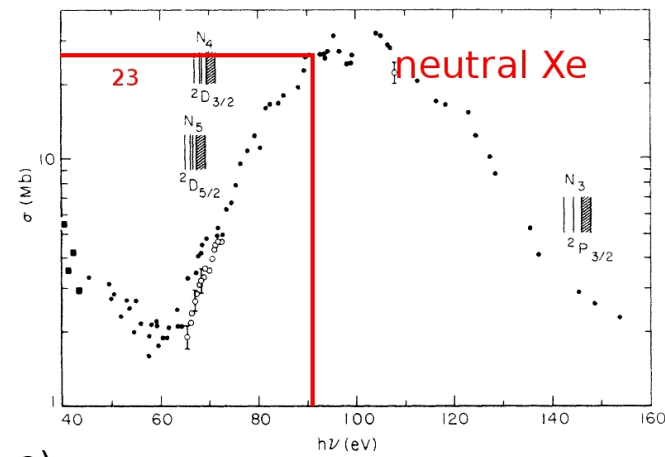

a)
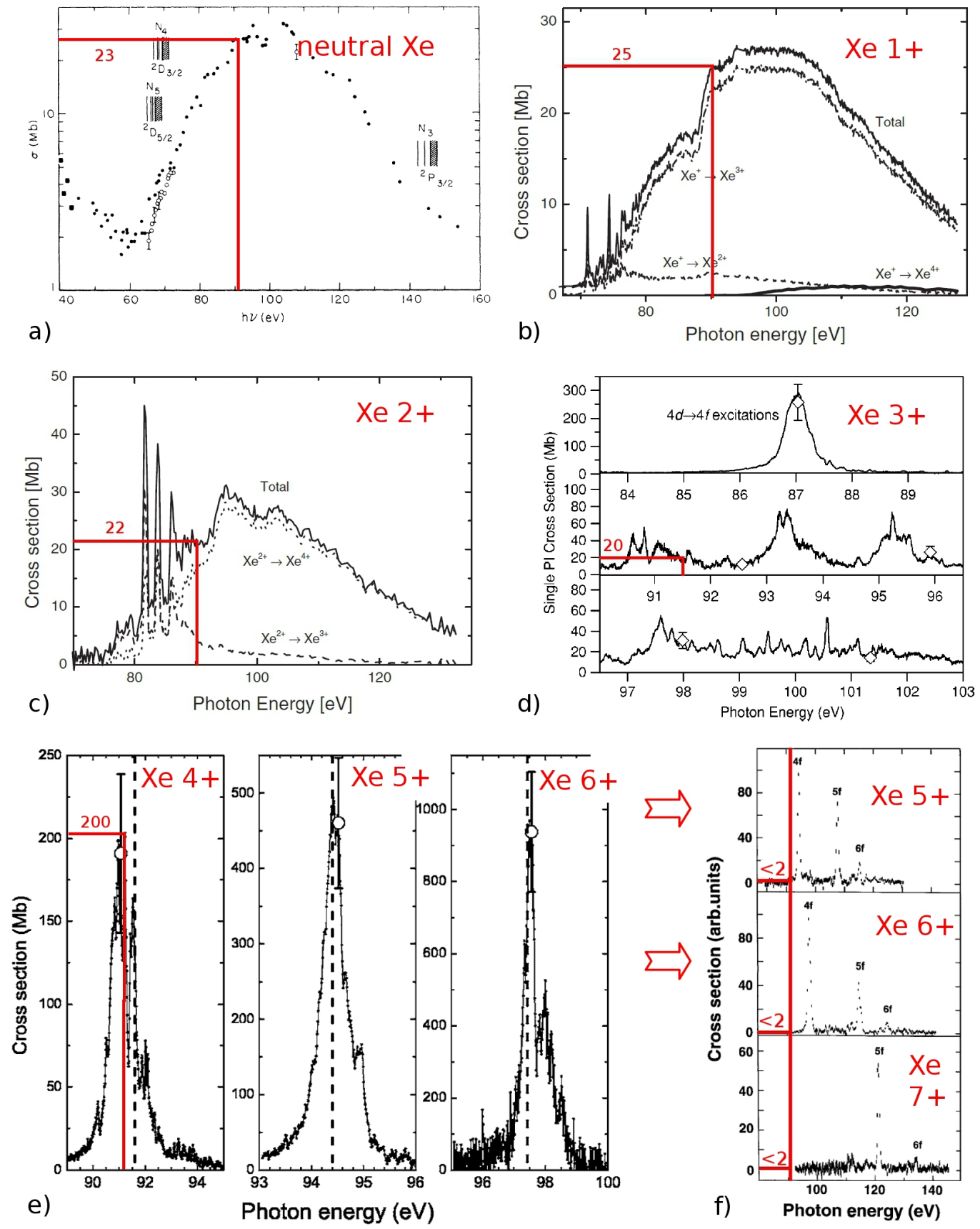

Figure 2.11: a)-f) Experimentally obtained absorption cross-sections of atomic xenon and its ions up to $\mathrm{Xe}^{7+}$ in the XUV spectral regime. From [62, 63, 64, 65, 66, 67]. A transition from a broad continuum-like absorption resonance for neutral xenon and lower charge states to sharp absorption resonances for higher charge states can be observed. The cross-section value at the actual photon energy in the current experiment of $91 \mathrm{eV}$ is indicated by red bars. At $\mathrm{Xe}^{4+}$ the excitation energy matches a strong resonance with up to 200 Mbarn. 
Absorption cross-sections of $\mathrm{Xe}^{q+}$ : The respective cross-section values at the actual photon energy in our experiment of $(91.1 \pm 1.4) \mathrm{eV}$ are indicated in Fig. 2.11 by the red lines. The obtained values are summarized in table 2.1. A closer examination of the absolute values of the absorption cross-section reveal a unique role of $\mathrm{Xe}^{4+}$ for irradiation with $91 \mathrm{eV}$. While from neutral up to triply charged xenon, the cross-section ranges between 20 and $25 \mathrm{Mbarn}$, (cf. Fig. 2.11 a-d, [62, 64, 65]), the resonant, autoionizing $4 d-4 f$ feature overlaps with the excitation energy at the maximal cross-section of 200 Mbarn (cf Fig. 2.11 e, [66]). As the resonant features shift towards higher energy for $\mathrm{Xe}^{5+}$ and $\mathrm{Xe}^{6+}$, the cross-section at $91 \mathrm{eV}$ remains much lower. Though no absolute values of measurements at this energy have been published, from the relative yields in Fig. $2.11 \mathrm{f}$, compared to absolute values for the respective $4 d-4 f$ resonances in Fig. 2.11 e, the cross-sections can be assumed to be less than $2 \mathrm{Mbarn}$. For $\mathrm{Xe}^{7+}$ only measured relative yields in the vicinity of $91 \mathrm{eV}$ are published together with calculations [67], but the absorption below the strong features is described to be flat and low, thus also values clearly below 2 Mbarn can be assumed.

As the $4 d$ shell is already an inner shell, ionization processes can lead to excited states with an inner-shell vacancy. For neutral xenon, the absorption of one $91 \mathrm{eV}$ photons results almost exclusively in doubly or triply charged ions, as one or even two Auger decays from the $5 s$ and $p$ shells follow the initial photo-ionization [71]. Absorption in the valence shell or radiative decays of the $4 d$ core hole on the other hand can be neglected. Auger processes follow the photo-ionization up to $\mathrm{Xe}^{2+}$, for higher charge states the energy difference available from the decay of a valance electron into the $4 d$ vacancy is no longer sufficient to emit further electrons, and eventually, no more electrons are present in the valance shell to conduct Auger decay. The partial cross-sections for neutral xenon are estimated from [71] to be less than 1 Mbarn for $0 \rightarrow 1+$ and 12.5 and 10.5 Mbarn for $0 \rightarrow 2+$ and $0 \rightarrow 3+$, respectively. For $X e^{2+}$ and $X e^{3+}$, the partial cross-sections can be extracted from Fig. $2.11 \mathrm{~b}$ and c.

Xenon gas in $90 \mathrm{eV}$ pulses: The peculiar ionization properties of xenon close to $90 \mathrm{eV}$ also manifested themselves in initial experiments on xenon gas at the FLASH free-electron laser [51]. The experiment, which was carried out at a photon energy of $93 \mathrm{eV}$ revealed surprisingly high charge states as indicated in Fig. 2.12. At an intensity of $8 \cdot 10^{16} \mathrm{~W} / \mathrm{cm}^{2}$, charge states up to $\mathrm{Xe}^{21+}$ were observed.

A total energy absorption of more than $5 \mathrm{keV}$ must be absorbed by a single atom to reach this charge state (cf. Fig $2.12 \mathrm{~b}$ ), seven photons are necessary to ionize ground state $\mathrm{Xe}^{20+}$. In order to produce the charge state $\mathrm{Xe}^{11+}$, which was the highest charge state obtained

\begin{tabular}{|l|l|l|l|l|}
\hline$q$ & $\begin{array}{l}\text { total } \sigma \\
{[\text { Mbarn }]}\end{array}$ & $\begin{array}{l}\sigma_{q \rightarrow q+1} \\
{[\text { Mbarn }]}\end{array}$ & $\begin{array}{l}\sigma_{q \rightarrow q+2} \\
{[\text { Mbarn }]}\end{array}$ & $\begin{array}{l}\sigma_{q \rightarrow q+3)} \\
{[\text { Mbarn] }}\end{array}$ \\
\hline \hline neutral & 23 & $<1$ & 12.5 & 10.5 \\
\hline $1+$ & 25 & 2 & 23 & 0 \\
\hline $2+$ & 22 & 4 & 16 & 0 \\
\hline $3+$ & 25 & 25 & 0 & 0 \\
\hline $4+$ & 200 & 200 & 0 & 0 \\
\hline $5+$ & $<2$ & $<2$ & 0 & 0 \\
\hline $6+$ & $<2$ & $<2$ & 0 & 0 \\
\hline $7+$ & $<2$ & $<2$ & 0 & 0 \\
\hline
\end{tabular}

Table 2.1: Total and partial xenon absorption cross-sections at $91 \mathrm{eV}$ 

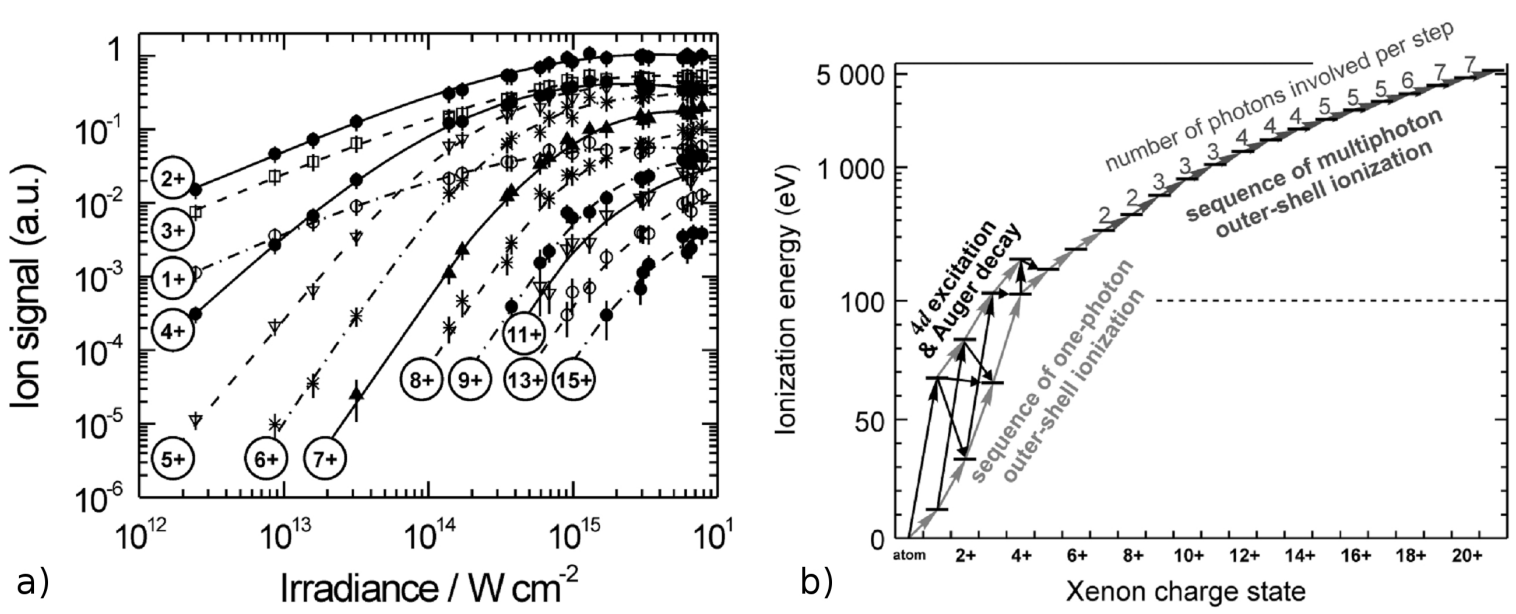

Figure 2.12: Ionization of xenon gas in intense $93 \mathrm{eV}$ pulses [51]. a) Relative ion signal intensities of $\mathrm{Xe}^{q+}$ as a function of intensity. b) Scheme of xenon energy levels and total ionization energy. For producing $\mathrm{Xe}^{21+}$ more than $5 \mathrm{keV}$ must be absorbed by a single atom. Seven photons are necessary to ionize ground state $\mathrm{Xe}^{20+}$, clearly indicating nonlinear processes.

in the present experiment, still at least 18 photons have to be absorbed in total, three are necessary to bridge the energy difference between $10+$ and $11+$. These observations put the results obtained in the last paragraph into question, that mostly linear processes should be expected. Full modeling of the ionization effects in xenon remains challenging due to the contribution of a large number of electrons and great difficulties to include electron-electron correlations correctly $[51,54]$.

\subsection{Clusters in intense laser pulses}

Clusters offer a way to investigate the organization and properties of matter from a fundamental point of view [72]. In particular gas phase clusters are widely used as ideal model systems to study the interaction between light and matter [73, 74, 29, 59, 22]. Compared to gas targets, clusters exhibit a high local density. On the other hand, they have less dissipation channels as bulk matter $[22,29]$. The easily scalable size allows for tuning their properties from molecular to bulk limit, which makes it possible to distinguish between intraand interatomic effects.

Rare gas clusters are weakly bound Van-der-Waals systems [72]. They exhibit the simple electronic structure of inert rare gas atoms which is hardly changed by the cluster surrounding [75]. The generation of rare gas clusters will be discussed in more detail in section 3.2.2.

In this experiment the interaction of matter with high intense XUV pulses is studied using rare gas clusters in the gas phase. This section covers the wavelength dependent phenomena occurring in a cluster compound when irradiated by an intense laser pulse, in particular the creation and dynamics of a nanoplasma. Concepts are introduced to describe the dynamics qualitatively. A brief overview will be given on previous experiments in intense short wavelength FEL pulses. 


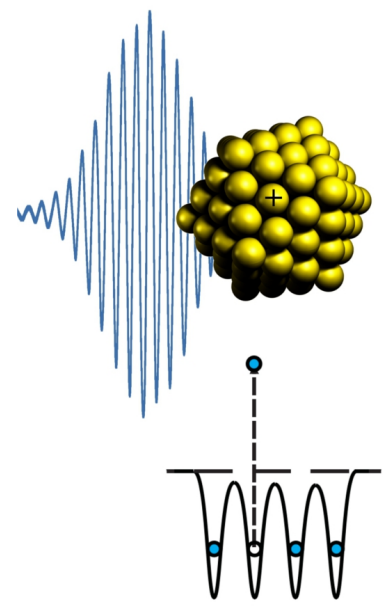

Cluster ionization, charge up
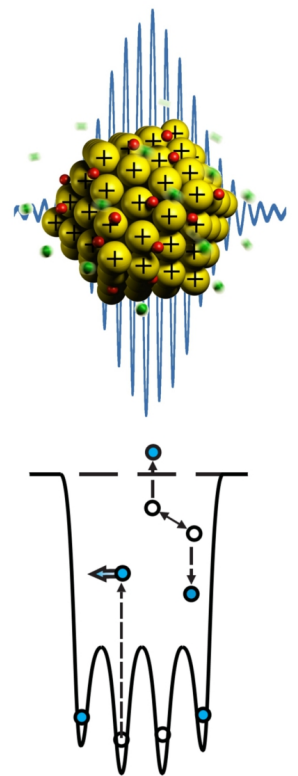

Electrons are trapped, nanoplasma
III) After the pulse

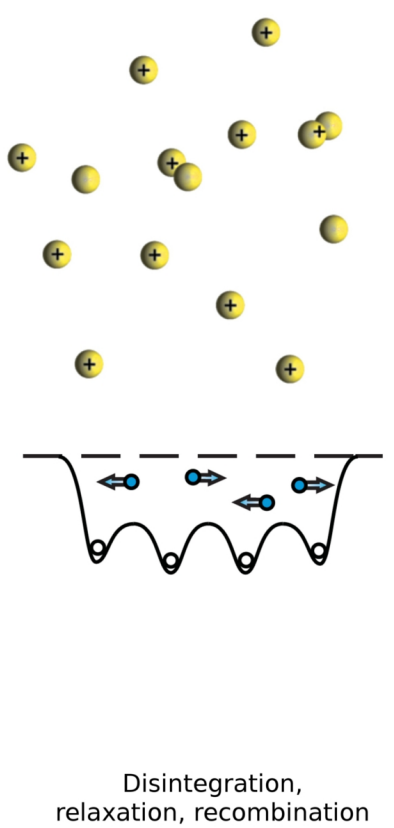

Figure 2.13: Description of laser cluster interaction in three phases: I) The light interacts with the atoms as if they were isolated. Electrons are ionized from the atomic potentials and leave the cluster. This is referred to as outer ionization. II) Electrons will be further inner ionized from atoms. But they are trapped in the increasing Coulomb potential of the cluster and a nanoplasma builds up. Only by subsequent plasma processes, electrons might gain sufficient energy to leave the cluster. III) When the pulse is over, disintegration, recombination and relaxation processes take place.

The concepts are taken from the literature [78, 77, 29, 75, 79]. The illustration of the cluster potentials is taken from the work of Arbeiter and Fennel [79].

\subsubsection{Clusters as model systems for laser-matter interaction}

Laser excitation of clusters in the infrared and visible range has introduced new possibilities to study and control ultra-fast many-particle dynamics [59]. The phenomena found in clusters can be roughly categorized as effects arising from the finite size of the system, from many-body dynamics, or from collective processes. The response of clusters to low intensity light fields has been used to study photoionization, relaxation and structural modification in finite-size quantum systems [76]. In highly intense IR pulses large-amplitude collective electron motion and violent explosion have been observed [74, 73]. Since the first experiments at FLASH [77], clusters have been also used to study processes and dynamics in intense short-wavelength pulses (see for example [22] and references therein).

The interaction with strong laser pulses, irrespective of the wavelength, will create a shortlived and dense nanoplasma [59]. The transient multi-electron dynamics in the nanoplasma, however will greatly differ with excitation energy. In particular in the short-wavelength range, they are still largely unexplored [75]. 
Three phases of cluster-laser interaction Ionization and disintegration of clusters proceed in several different steps on different time scales [22, 29]. The particular mechanisms and dynamics in the clusters depend on the excitation energy and intensity of the laser, but they also change with cluster size and atomic species. In order to describe a general scenario applicable to all conditions, one can employ a concept which describes the laser induced dynamics in clusters in three phases.

The three phases are sketched in Fig. 2.13.

I) At the beginning of the pulse the light interacts with the atoms as if they were isolated. Wavelength dependent ionization processes result in the emission of electrons, which leave the cluster. These electrons are referred to as outer ionized.

II) Further electrons are liberated from the individual atomic potentials, but depending on their kinetic energy, at some point they will be trapped in the increasing Coulomb potential of the cluster. The generation of inner ionized electrons confined to the cluster results in the buildup of a nanoplasma. By subsequent plasma processes, electrons might gain additional kinetic energy and outer ionize, i.e. leave the cluster.

III) When the pulse is over, the cluster disintegrates. Also recombination and relaxation processes might take place.

The concept of inner and outer ionization has been introduced by Last and Jortner [78]. Similar three-phase models have been used in the literature with slightly different definitions $[77,29,75,79]$. The scheme in Fig. 2.13 will be used in the subsequent section to describe the dynamics in the clusters, which happen on different time scales and may differ greatly using for example different lasers. In addition to the wavelength dependent onset of the ionization, the influence of the plasma environment on the ionization processes and possible heating mechanisms have to be considered in particular. Ultimately the plasma properties determine the progress and mechanisms of the expansion and a possible contribution from recombination processes.

\subsubsection{Properties and dynamics of a nanoplasma}

Onset of plasma formation: Wavelength dependent ionization mechanisms in atoms have already been discussed in section 2.2. As the laser pulse will couple independently to the atoms in phase 1, the findings of section 2.2 apply also to the onset of ionization in clusters. In the infrared regime, only above a certain intensity threshold tunnel ionization will occur. Lower laser intensities are required in the visible and UV range, where a small number of photons or even single photons can ionize valence electrons.

From the pure tunnel regime up to multi-photon ionization of first valence shells, electrons are mainly liberated into the continuum without any additional kinetic energy. This results in an immediate onset of the plasma formation.

Towards higher photon energies, single photon absorption already occurs at low intensities. The released photoelectrons take away the excess energy as kinetic energy. If inner-shell electrons are photoionized, the decay of the inner-shell vacancy can result in the emission of further electrons. Depending on the kinetic energy of the electrons, the formation of a plasma will be delayed. By a simple electrostatic approach [80] of a charged sphere, the number of electrons can be estimated which can leave the cluster potential before electron emission of 
the cluster is fully frustrated. The effective kinetic energy of an electron in the increasing Coulomb potential and the total number of outer-ionized electrons $n_{e, \text { out }}$ are given by [80]

$$
\begin{array}{r}
E_{k i n, \text { out }}=\left(h \nu-I_{p}\right)-\frac{e^{2}}{4 \pi \epsilon_{0}} \sum_{i \neq j} \frac{q_{i}}{r_{i j}} \\
\Leftrightarrow n_{e, \text { out }}=\left(h \nu-I_{p}\right) \frac{4 \pi \cdot \epsilon_{0} \cdot R}{e^{2}} .
\end{array}
$$

In order to characterize the properties of the developing nanoplasma, useful concepts can be borrowed from plasma physics.

Plasma coupling: Low density plasmas can be treated as independent particles, which experience occasional collisions. In a high density plasma, the ions differ clearly from isolated systems. The electronic structure will be strongly perturbed and many-body collisions become a dominant process [34]. In the case of laser-irradiated clusters, the rapidly developing plasma will have the density of a solid and higher.

A plasma can be characterized by the degree of coupling between its constituents [34]. The coupling parameter $\Gamma_{A B}$ is defined as the ratio of mean potential and kinetic energy, with $A, B$ being two plasma species, for example electrons and ions. The electron-electron coupling parameter $\Gamma_{e e}$ can be calculated as [81]

$$
\Gamma_{e e}=\frac{V_{e e}}{k_{B} T_{e}}
$$

with the average thermal energy of the electrons $k_{B} T_{e}$ and the electrostatic energy between two neighboring electrons $V_{e e}=e^{2} /\left(4 \pi \varepsilon_{0} R_{e}\right)$. The radius of the so called electron sphere can be calculated from the electron density to $R_{e}=\left(4 \pi / 3 n_{e}\right)^{-1 / 3}$. The coupling parameter between electrons and ions $\Gamma_{i e}$ can be calculated from $\Gamma_{e e}$ to

$$
\Gamma_{i e}=q \cdot \Gamma_{e e}^{\frac{3}{2}}
$$

The coupling parameters are therefore functions of the electron density and temperature. A strongly coupled plasma arises in the limit of high density and low temperature $\left(\Gamma_{i e} \geq 1\right.$ and $\left.\Gamma_{e e} \geq 0.1[81]\right)$. This limit is also characterized by a short Debye length $\lambda_{D}$ with

$$
\lambda_{D}=\sqrt{\frac{\varepsilon_{0} k_{B} T_{e}}{e^{2} n_{e}}} .
$$

The Debye length denotes the distance over which charge fluctuations are screened by the electrons in the plasma. In cluster plasmas with a density in the order of solid density (about $10^{23} \mathrm{~m}^{-3}$ ), considering a kinetic energy of the electrons of $1 \mathrm{keV}$, a typical Debye length is in the order of $\lambda_{D} \approx 5 \AA$.

The degree of degeneracy $\gamma$ of a plasma can be calculated as the ratio of Fermi temperature to electron temperature. The Fermi temperature reads

$$
T_{F}=\frac{\hbar^{2}}{2 m_{e}}\left(3 \pi n_{e}\right)^{\frac{2}{3}},
$$

therefore the degeneracy parameter $\gamma$ yields

$$
\gamma=\frac{T_{F}}{T_{e}}=\frac{\hbar^{2}\left(3 \pi n_{e}\right)^{\frac{2}{3}}}{2 m_{e} T_{e}},
$$

A plasma with $\gamma>1$ can be considered metallized. 

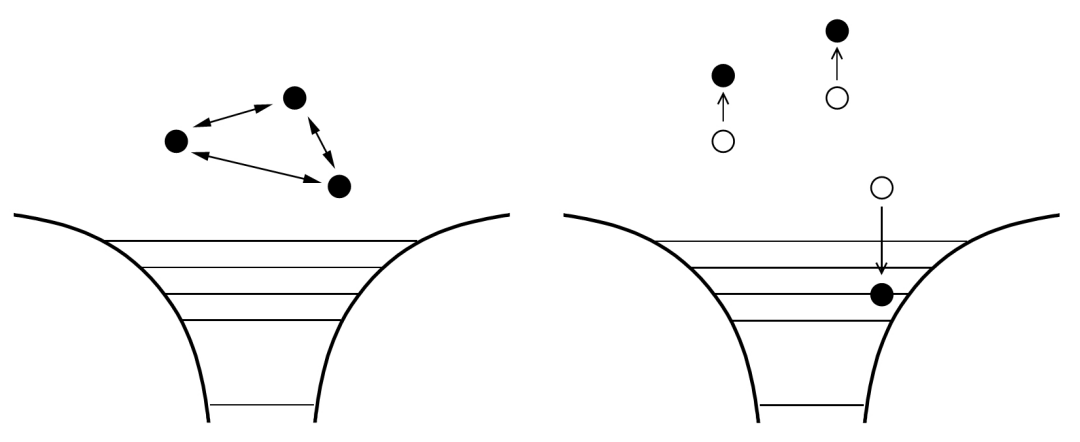

Figure 2.14: Many-body recombination processes can become significant in strongly coupled plasmas [81]. An ion interacts with several electrons and one electron is transferred into a bound state. The difference in energy and momentum are taken away by the other electrons.

Energy shifts and collision processes: In a plasma environment, three key effects have to be considered [34].

- Perturbation of atomic states,

- Screening of long range forces,

- Changes in atomic transition rates.

These effects are in particular important in dense and strongly coupled cluster plasmas. The local electric field of the cluster, in particular the vicinity of ions and the presence of plasma electrons leads to suppression of the interatomic barriers in the plasma [82, 83]. These effects are also referred to as plasma screening [83, 34]. In first approximation, plasma screening leads to a constant shift of all energy levels and therefore to a decrease of the binding energy of electrons [34]. In extremely dense plasmas, barrier suppression can even result in direct inner ionization of valence electrons.

Also all collision related processes are of special significance in cluster plasmas. Collisional excitation and ionization arise in particular in the presence of heating mechanisms which increase the kinetic energy of the electrons [59]. But also their counterparts, collision induced decay processes and many-body recombination play an important role in the dynamics and final states of the laser-cluster interaction. A sketch of many-body recombination is given in Fig. 2.14 [81]. An ion interacts with several electrons at the same time. One electron is transferred into a bound state, while the excess energy and momentum are taken away by the other electrons. Another collision-related process is collisional heating by Inverse Bremsstrahlung (IBS). IBS is the dominant non-resonant heating process for long wavelength pulses $[84,29,59]$. The underlying mechanism is illustrated in Fig. 2.15 a [84]. The process is termed Inverse Bremsstrahlung because the electrons obtain the increase in kinetic energy directly from the acceleration in the light field. The acquired kinetic energy of free electrons in a single laser cycle has been introduced in section 2.2.1 as the ponderomotive potential (cf. Eq. 2.76). Without facing any collisions, a free electron would follow the laser field but it would loose again the kinetic energy at the end of the pulse. Only by collisions with ions a phase difference between the field and the electron motion is introduced and the total kinetic energy of the electron increases. 


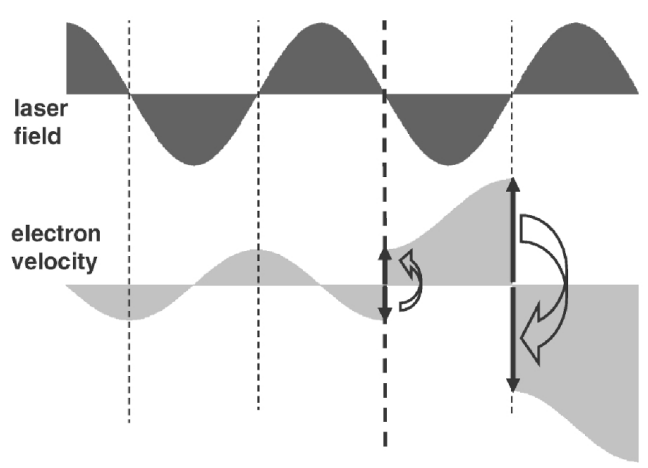

a)

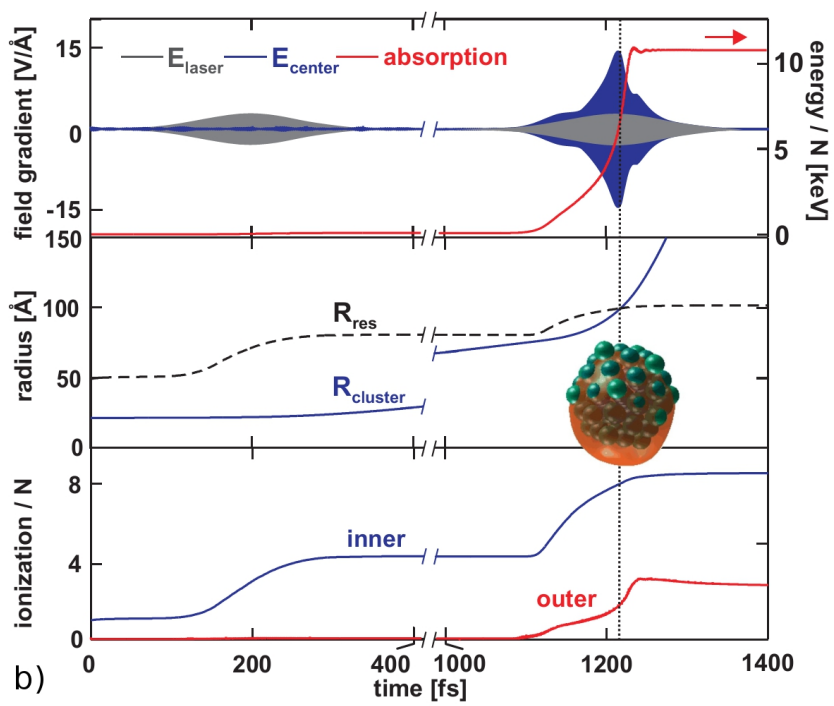

b)

Figure 2.15: Two key mechanisms can be made responsible for the effective energy transfer into clusters from intense IR fields, non-resonant and resonant heating of the electrons. a) Sketch of the time-evolution of inner ionized electrons in the laser field. Due to elastic collisions with ions, the electrons are redirected (here by $180^{\circ}$ ) and receive a phase shift in respect to the laser field. Energy is transferred directly from the light field to the electrons, therefore this process is referred to as inverse bremsstrahlung (IBS) [84]. b) Time structure of resonant excitation of silver clusters in an IR double pulse [85]. If the laser frequency matches the frequency of the surface plasmon, a large amount of energy can be resonantly coupled into the cluster [59]. See text for details.

Optical phenomena in finite plasmas: At infrared and visible frequencies, the light is not able to penetrate a dense plasma and will be completely reflected. This phenomenon is referred to as opacity of the nanoplasma. Full screening is preserved as long as $\omega_{\text {las }}<\omega_{\text {plas }}$ with the laser frequency $\omega_{\text {las }}$ and the plasma frequency

$$
\omega_{\text {plas }}=\sqrt{\frac{e^{2} n_{e}}{m_{e} \varepsilon_{0}}} .
$$

Therefore, in the beginning of an IR pulse, when the cluster plasma is still dense, electron heating due to IBS only plays a role in the surface region of the cluster [59].

At a distinct laser frequency $\omega_{l a s}=\omega_{\text {mie }}$ with

$$
\omega_{\text {mie }}=\frac{1}{\sqrt{3}} \omega_{\text {plas }}=\sqrt{\frac{e^{2} n_{\text {ion }}}{3 m_{e} \varepsilon_{0}}},
$$

a collective electron motion of the electron cloud can be excited. The so called surface or Mie plasmon is responsible for the finding in intense IR pulses that clusters can absorb more energy per atom than atoms and bulk matter $[73,59]$. The extreme energy transfer from the light field into the plasma arises from a resonant excitation of large-amplitude oscillations of the electron cloud in respect to the ionic background. The coupling constant of this collective motion and therefore the resonant frequency depends on the ionic density $n_{\text {ion }}$. In the typical case of $800 \mathrm{~nm}$ IR pulses, the laser frequency is lower than the resonant frequency at the beginning of the interaction. After a distinct time the cluster is expanded up to the critical density $n_{\text {ion,res }}$, where the frequency of the Mie-plasmon matches the laser frequency and resonant absorption will occur. Therefore Mie-plasmons can not be excited in a single very 
short laser pulse. But the expansion time up to resonant density can be probed by either increasing the pulse length [86] or in a pump-probe scheme [85]. Fig. 2.15 b illustrates the time structure of the plasmon excitation in small silver clusters using two laser pulses with 1 ps delay [85]. The red line in the uppermost graph shows the time resolved analysis of the calculated energy absorption. The decrease in ion density and the overlap with the resonant density is displayed in the second panel in terms of the cluster radius. The progress of inner and outer ionization can be traced in the third panel. Due to the resonant excitation an increase in the total number of activated electrons due to collisional ionization but also in the outer ionization due to the efficient heating can be observed.

For frequencies $\omega_{\text {las }} \gg \omega_{\text {plas }}$, the excitation of resonant motion can be excluded and the laser field will penetrate the cluster plasma from the beginning. In the limit of a finite metallized plasma $(\gamma>1)$ the optical properties, which determine the propagation, can be described by the dielectric function of a metallic sphere $[44,50]$

$$
\epsilon\left(R, \omega_{\text {las }}\right)=1+\chi_{0}-\frac{\omega_{\text {plas }}^{2}}{\omega_{\text {las }}^{2}+i \cdot \omega_{\text {las }} \cdot \nu(R)} .
$$

In this model, the response of the bound electrons is represented by $1-\chi_{0}$, condensed into the real-valued background susceptibility $\chi_{0}$, while the response of the electron cloud is described by the fraction $\omega_{\text {plas }}^{2} /\left(\omega_{\text {las }}^{2}+i \omega_{\text {las }} \nu\right)$ with the electron-ion collision frequency $\nu[87]$.

Expansion mechanisms: With the end of the pulse, the deposition of energy into the cluster stops. However, the energy will be further redistributed, for example through collisions of electrons and ions leading to further collisional ionization or to recombination and relaxation.

The self-consistent redistribution process is determined by the plasma properties, in particular the net charge, electron density and electron temperature [74]. Within the expansion, energy is transferred into the motion of ions. The increasing kinetic energy of the ions results

- from the net charge of the cluster, i.e. Coulombic repulsion and/or

- from the kinetic energy of the quasi-free plasma electrons.

These sources of ionic kinetic energy define the limits of the two key expansion mechanisms, pure Coulomb explosion and hydrodynamic expansion. If many electrons are outer ionized, the net charge results in Coulomb explosion. If on the other hand a considerable number of activated electrons is confined in a nanoplasma, expansion driven by hydrodynamic forces will dominate $[88,89]$. The latter process results in cooling of the electron cloud and electrons may recombine. Depending on the temporal evolution of ion density and electron temperature, recombination can be an important process accompanying hydrodynamic expansion [79]. Typically both mechanisms contribute to the disintegration of clusters [90].

The final ion charge state and energy distribution can give information on the underlying expansion process. However, deducing the dominant expansion mechanism from the distributions of ion kinetic energies is a much-discussed topic $[91,92,88,90,89,93,73,94,79]$. A major difficulty of interpreting the kinetic energy distribution of ions arises from the averaging over many different intensities in the focus profile and different cluster sizes in the probed ensemble of clusters [95, 96]. The interrelationship between kinetic energy and charge state of ions and implications for the expansion process will be further discussed in the analysis of ion spectra from single clusters of defined size and power density in section 4.4.4. 

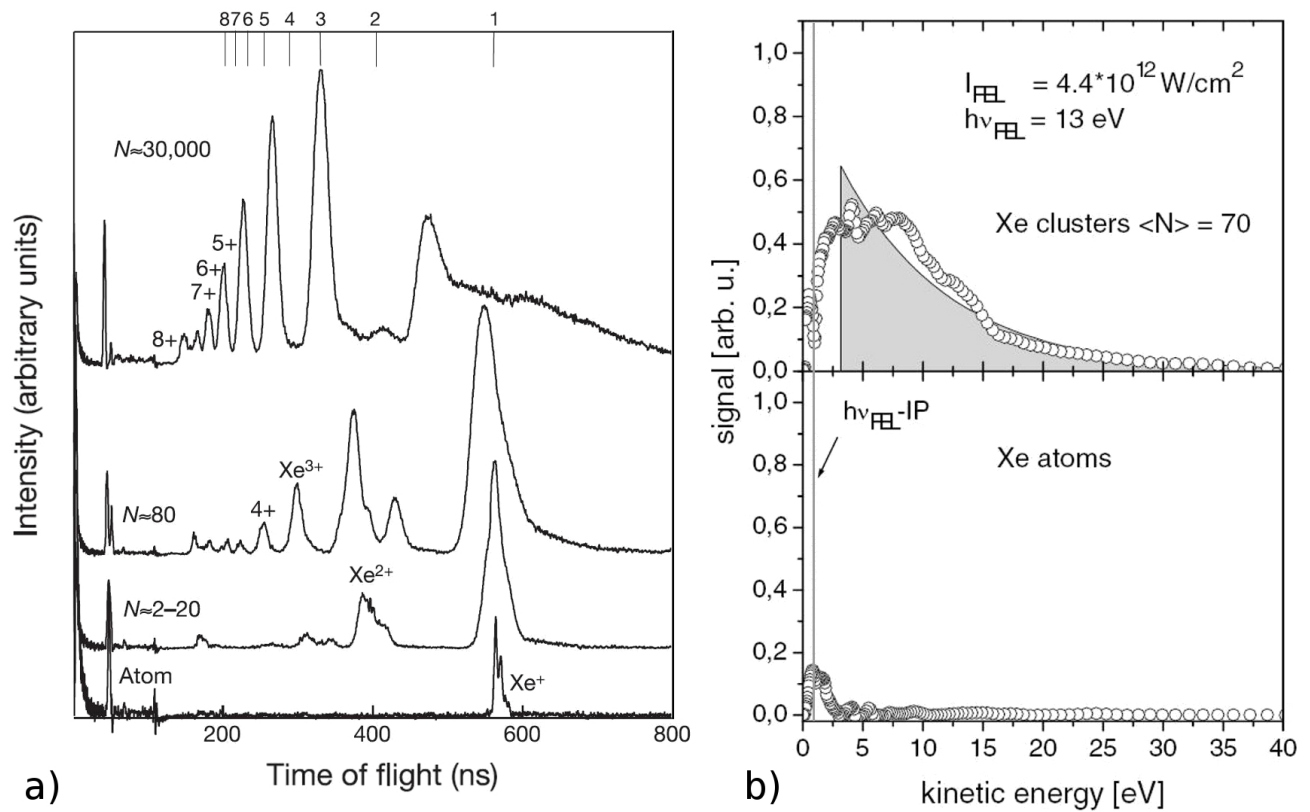

Figure 2.16: Xenon clusters in intense VUV pulses. a) Ion spectra of atomic gas and different cluster sizes. At $100 \mathrm{~nm}$ wavelength charge states up to $\mathrm{Xe}^{8+}$ are produced in xenon clusters with $\approx 20000$ atoms [77]. b) Electron spectra of small xenon clusters reveal a Boltzmann-like structure, pointing towards efficient heating processes [97].

The subsequent paragraph gives an overview of previous experiments on clusters in shortwavelength pulses from the FLASH free-electron laser in the range from $10 \mathrm{eV}$ to $100 \mathrm{eV}$ photon energy and the linear coherent light source LCLS around $1 \mathrm{keV}$. Interpretations of the effects according to the current understanding are given with an emphasis on recombination and expansion processes.

\subsubsection{Rare gas clusters in intense short-wavelength pulses}

Results in the VUV-range: Rare gas clusters have been the first targets to be studied in the light of the FLASH free-electron laser [77]. The results are displayed in Fig. 2.16 a. At a photon energy of $13 \mathrm{eV}$ and moderate power densities up to $8 \cdot 10^{12} \mathrm{~W} / \mathrm{cm}^{2}$ [22] the ion spectra of atomic xenon gas and clusters from a few up to $10^{4}$ atoms were measured. For largest cluster sizes charge states up to $\mathrm{Xe}^{8+}$ were observed, while atomic gas could be only singly ionized $\left(I_{p}=12.1 \mathrm{eV}\right)$. Fig. $2.16 \mathrm{~b}$ shows electron spectra of small xenon clusters at the same photon energy. They revealed a Boltzmann-like distribution of kinetic energies up to $40 \mathrm{eV}$ [97]. Reference measurements on atomic xenon gas show only small kinetic energies of the photoelectrons below $1 \mathrm{eV}$ (lower panel of Fig. $2.16 \mathrm{~b}$ ). Therefore, the kinetic energies of the electrons indicate heating of the electrons in the cluster environment.

In particular heating processes had been expected to be insignificant at $100 \mathrm{~nm}$ wavelength from heating rates valid in the infrared [74]. The results inspired several subsequent theoretical studies leading to improvements of the underlying models [98, 99, 100, 81, 101]. According to the current understanding, the efficient energy absorption of clusters in VUV pulses results from IBS heating which is still efficient in a strongly coupled plasma at $100 \mathrm{~nm}$ wavelength. Generation of singly charged ions happens through single photon absorption. Due to barrier suppression, also $\mathrm{Xe}^{2+}$ is generated in the cluster environment. In the strongly coupled 

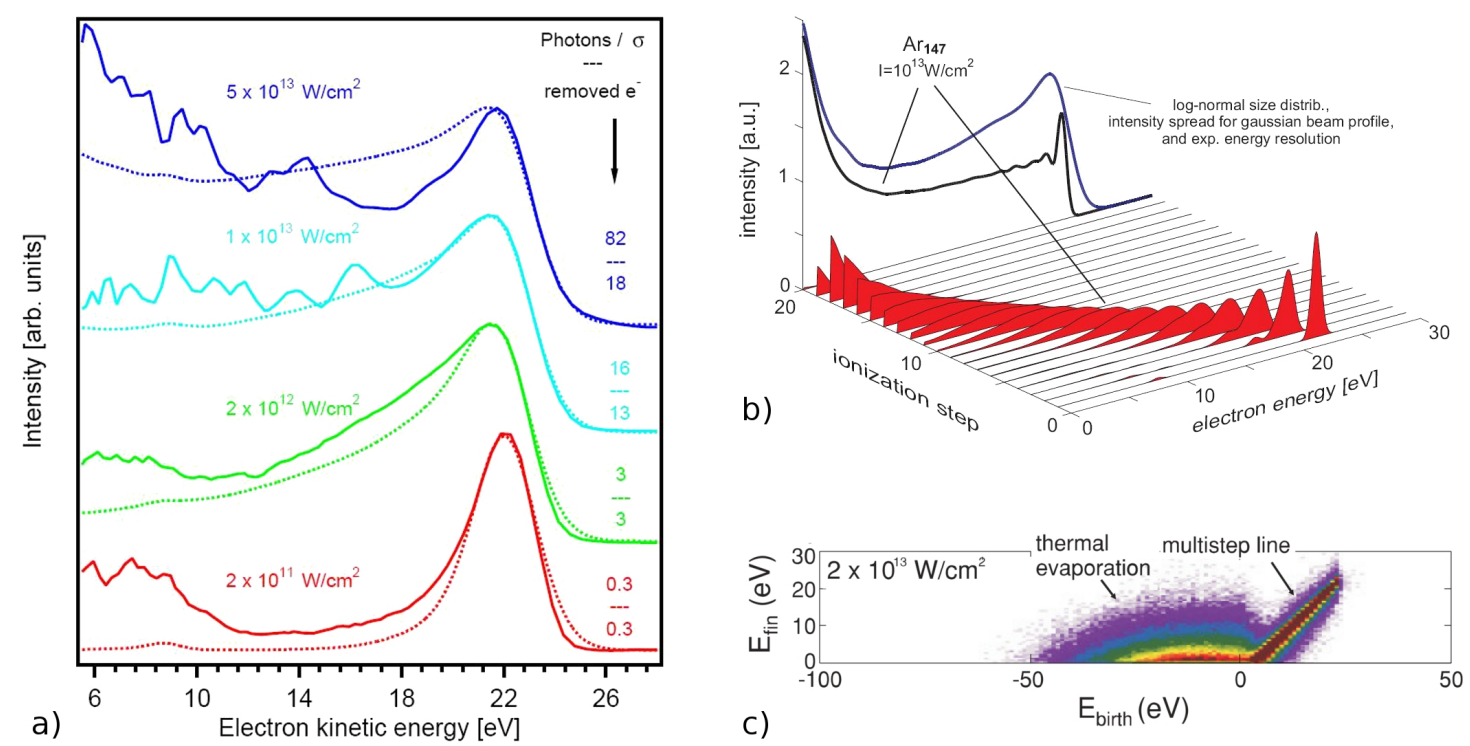

Figure 2.17: Cluster ionization at $38 \mathrm{eV}$ can be described by sequential multistep absorption. a) Electron spectra from argon clusters irradiated with different FEL power densities $\left(E_{\text {phot }}=38 \mathrm{eV}\right)$ reveal a strong feature at $22 \mathrm{eV}$, equivalent to the atomic photo line. With increasing power density, a plateau-like structure towards lower kinetic energies appears [102]. b) The plateau structure can be traced in a simple Monte Carlo model calculation as a sequential absorption of photons [102]. The kinetic energy of the sequence of outer ionized electrons decreases, adding up to plateau-like electron spectra. c) The deviation towards lower kinetic energies from the simple model at highest intensities can be explained as contribution from thermal plasma electrons. The energy correlation analysis between final kinetic energy of electrons and their single particle energy at the instant of birth reveals evaporation of thermalized electrons from a deep Coulomb potential [103].

plasma, three- and many-body collisions become very frequent processes, allowing for efficient heating of the electrons and subsequent collisional ionization. The self-amplifying effect of barrier suppression resulting from the ionized cluster environment and subsequently increased collisional ionization rates is termed ionization ignition [99]. Also an additional heating mechanism based on a cyclic process of many-body recombination and reabsorbtion of photons has been proposed [81].

Electron and ion spectra of XUV-excited clusters: In contrast to the results in the VUV range, where IBS heating plays still a major role, first experiments at $38 \mathrm{eV}$ photon energy revealed negligible heating of electrons from the light field [102]. Electron spectra of small argon clusters $(N \approx 100)$ obtained at different FEL intensities are displayed in Fig. 2.17 a. The prominent feature for all intensities is the photo line of argon at $h \nu-I_{p}=$ $22 \mathrm{eV}$. With increasing intensity a plateau-like structure towards smaller kinetic energies evolves. This plateau constitutes the characteristic feature of multistep ionization. This process denotes the sequential absorption of single photons and emission of electrons with stepwise less kinetic energy due to the increasing Coulomb potential (cf. Fig. 2.17b). Full frustration of outer ionization is reached for a distinct number of ionization steps, depending on the difference between photon energy and ionization potential (cf. also Eq. 2.77).

Only when this number is exceeded, a nanoplasma builds up and the confined electrons can thermalize by collisions. As plasma heating processes are negligible in the XUV range, the temperature of the electron cloud is determined by the excess energy from the photoionization process, which is the only supply of kinetic energy. This concept is termed ionization heating 

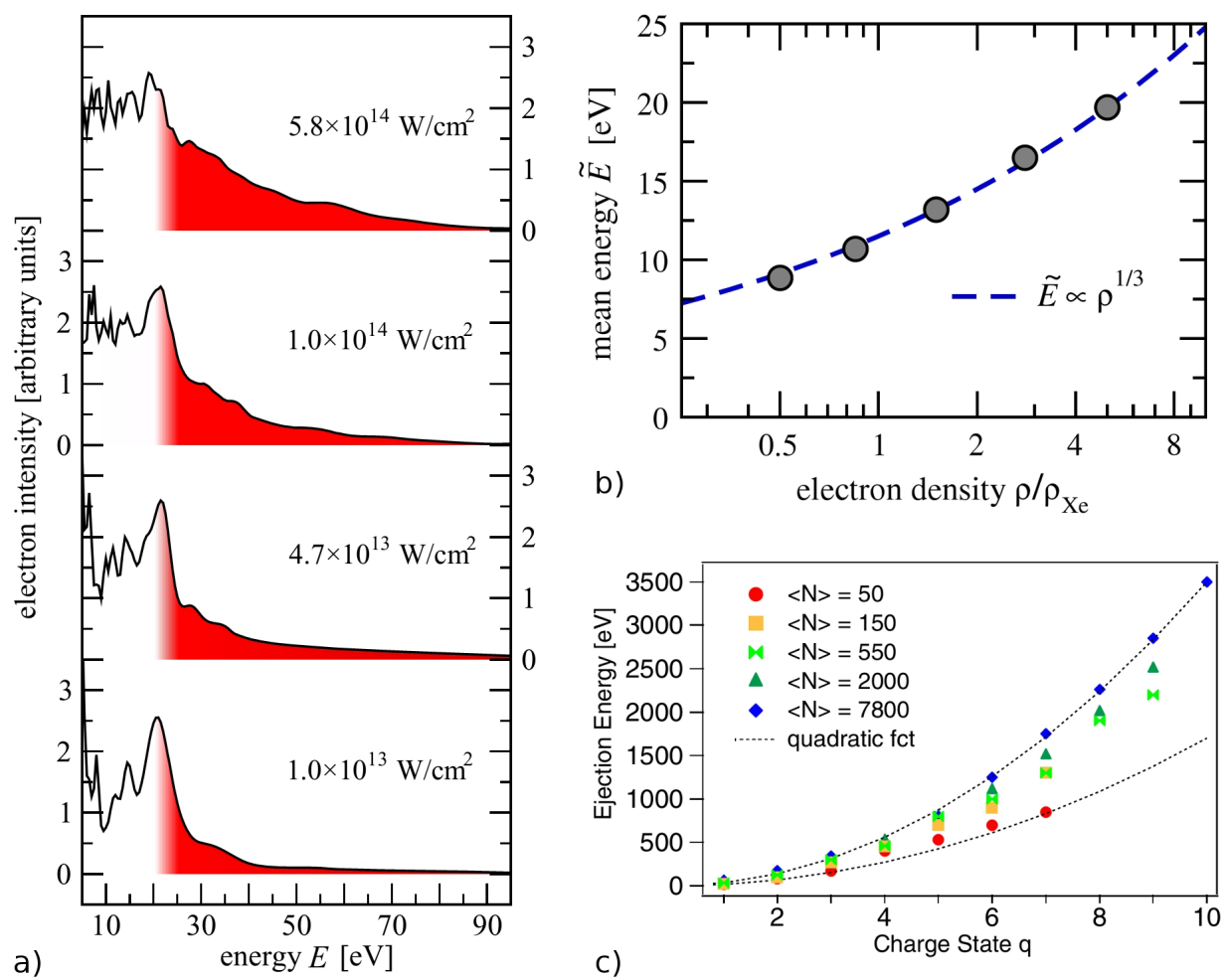

Figure 2.18: a) Electron spectra of xenon clusters with $\approx 2000$ atoms reveal a combination of a multistep feature and a thermal distribution. The clusters were irradiated in $90 \mathrm{eV}$ pulses at different FEL intensities [80]. b) The mean electron energy increases with the density of the electrons and therefore with the number of photoionization processes. c) Quadratic increase of the central kinetic energy per charge state. Xenon clusters with different sizes from $\approx 50-7800$ atoms were irradiated in $90 \mathrm{eV}$ pulses with an intensity of $5 \cdot 10^{14} \mathrm{~W} / \mathrm{cm}^{2}$ [94].

[103]. The tail of the thermalized electron distribution can be evaporated from the cluster, as displayed in Fig. 2.17 c. This contribution from plasma electrons, which overcome a Coulomb potential as deep as $100 \mathrm{eV}$ [103] explains the increase towards lower kinetic energies in the uppermost electron spectrum of Fig. 2.17 a.

The interplay between multistep and thermal emission of electrons became even more prominent in experiments on xenon clusters at $90 \mathrm{eV}$ photon energy [80]. The photoelectron spectra of clusters with $\approx 2000$ atoms for different FEL intensities are displayed in Fig. 2.18 a. The spectrum at lowest intensities resembles the spectra of argon clusters at $38 \mathrm{eV}$. At $90 \mathrm{eV}$ excitation energy, the first innershell of xenon is mainly addressed by photoionization. The $4 d$ photo line at $20 \mathrm{eV}$ and the Auger line at $32 \mathrm{eV}$ are accompanied by a multistep plateau towards lower kinetic energy. With increasing intensity a prominent second component with a Boltzmann-like distribution arises in the electron spectra. Due to the high cross-section of xenon (cf. also section 2.2.2) a plasma with supra-atomic density evolves. At an intensity of $6 \cdot 10^{14} \mathrm{~W} / \mathrm{cm}^{2}$ the distribution of thermally evaporated electrons reaches up to $90 \mathrm{eV}$ kinetic energy. By fitting the thermal component of the energy distribution, the increasing density and average kinetic energy of the electrons were extracted. The increase of the mean energy with electron density, which is presented in Fig. $2.18 \mathrm{~b}$, is again a signature of the dominant contribution of ionization heating to the plasma temperature.

The ionization and expansion dynamics of xenon clusters in $90 \mathrm{eV}$ pulses were also studied by 
means of ion spectroscopy [94]. Clusters with different sizes from $\approx 50-7800$ atoms irradiated with an intensity of $5 \cdot 10^{14} \mathrm{~W} / \mathrm{cm}^{2}$ revealed charge states up to $\mathrm{Xe}^{10+}$ with kinetic energies of the ions reaching $3.5 \mathrm{keV}$. A quadratic increase of the central ion kinetic energy with the charge state was found, as displayed in Fig. $2.18 \mathrm{c}$. Simulations of the ion spectra based on a simple electrostatic model indicate radial changes in the average charge state. Therefore, the kinetic energy distributions of the ions were interpreted as a Coulomb exploding outer shell and a plasma core which expands hydrodynamically.

Redistribution of charges within the cluster and effective recombination of the fully screened cluster core could also be traced in experiments using doped clusters [31]. In clusters with a xenon core and an argon shell, irradiated with $90 \mathrm{eV}$ pulses, only highly charged argon was detected. The xenon atoms in the core are expected to contribute most of the nanoplasma electrons, as the ionization cross section is 10 times larger than for the argon shell. The absence of higher xenon charge states in the ion spectra indicates a strong recombination of the screened cluster core.

Signatures of different expansion mechanisms: The structure of the electron spectra obtained in the XUV range emphasizes the importance of the element specific electronic structure for the ionization and electron emission processes. The main features reflect the binding energies of the electrons while the difference between binding energy and excitation energy predefines the onset of plasma formation. On the other hand, the absorption crosssections are responsible for the plasma density at a certain FEL intensity. Both together, the net charge on the cluster and the plasma density, ultimately determine the contributing expansion mechanisms [79].

The transition from hydrodynamic expansion to Coulomb explosion for increasing energetic distance between excitation energy and accessible atomic resonances in the short wavelength regime have been studied theoretically [79]. The respective expansion dynamics and their signature in electron and ion spectra are displayed in Fig. 2.19. Argon clusters with $N=923$ were simulated for excitation with $20 \mathrm{eV}, 38 \mathrm{eV}$, and $90 \mathrm{eV}$ photon energy. For comparability of the three cases, the total amount of absorbed energy was held constant. A simple frustration parameter $\alpha$ is introduced, which is defined as the ratio of activated (=inner+outer ionized) to critical number of electrons which can leave the cluster (cf. Eq. 2.77)

$$
\alpha=\frac{n_{e, t o t a l}}{n_{e, \text { out }}} .
$$

It denotes the neutrality of the plasma and was found to be a good measure to specify the dominant expansion mechanism. The temporal evolution of the cluster explosion as increasing distance in between the six geometric shells of the cluster with 923 atoms is displayed in Fig. 2.19 a. In the right panel, for $\alpha \approx 1$, the clusters undergo pure Coulomb explosion, as all activated electrons are able to leave the cluster. In contrast, the left panel for a value of $\alpha \approx 100$ indicates a plasma-driven cluster expansion, with an ejection of the outer shells due to hydrodynamic forces. For values in between, as displayed in the middle panel for $\alpha \approx 10$, a combination of both processes will appear, where the inner part of the cluster is efficiently screened and an outer shell can explode off.

The graphs in Fig 2.19 b show, that the transition from hydrodynamic expansion to Coulomb explosion can be traced in the electron spectra. While the Boltzmann-like distribution of thermally evaporated electrons corresponds to hydrodynamic expansion, a multistep feature is accompanied by Coulomb explosion. The simulations were also analyzed in terms of the kinetic energy distributions of the ion charge states, the results are displayed in Fig $2.19 \mathrm{c}$. 
$20 \mathrm{eV}, 2.5 \times 10^{12} \mathrm{~W} / \mathrm{cm}^{2}$ $\alpha=98$
$38 \mathrm{eV}, 1.5 \times 10^{13} \mathrm{~W} / \mathrm{cm}^{2}$

$\alpha=10.9$
$90 \mathrm{eV}, 5 \times 10^{13} \mathrm{~W} / \mathrm{cm}^{2}$

$$
\alpha=1.1
$$

a) shell resolved expansion:
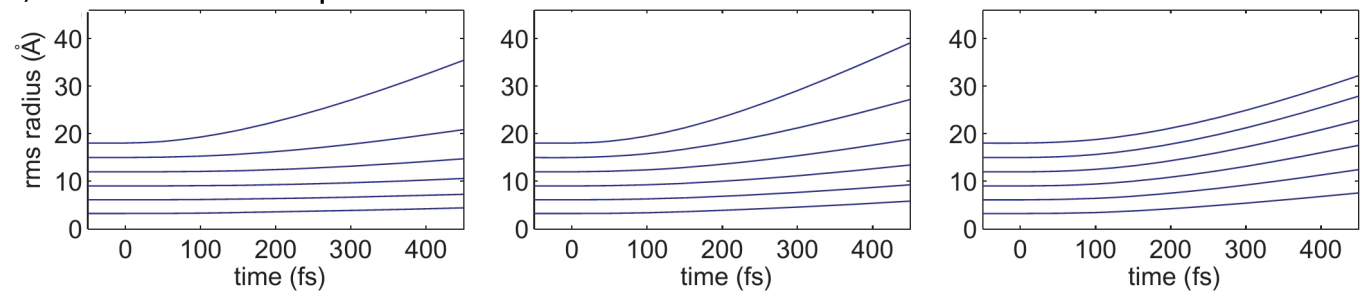

b) kinetic energy distributions of electrons:
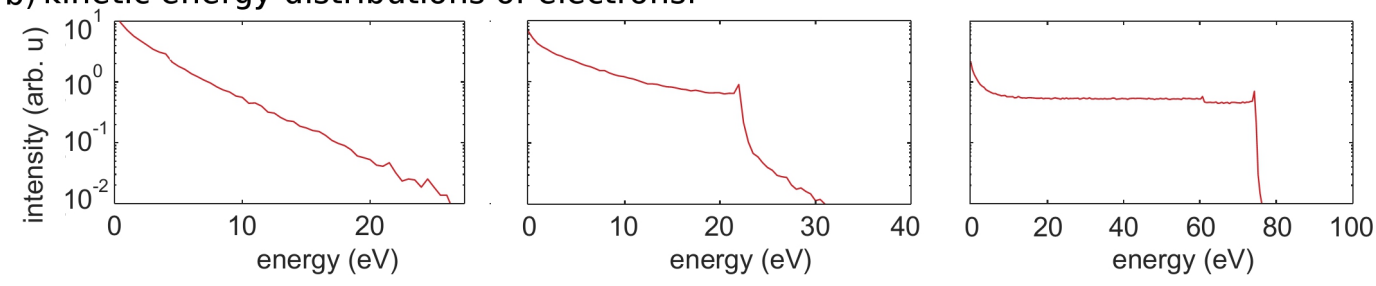

c) charge state resolved ion energy distributions:
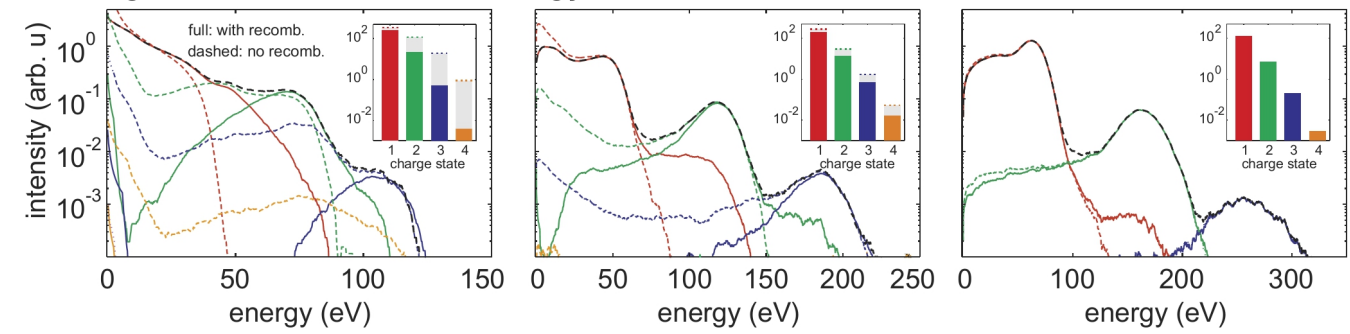

Figure 2.19: Calculated a) expansion dynamics, b) electron spectra and c) ion spectra of argon clusters $(N=923)$ in laser pulses with an excitation energy of $20 \mathrm{eV}$ (left column), $32 \mathrm{eV}$ (middle column) and $90 \mathrm{eV}$ (right column). A transition of the dominating expansion mechanism from hydrodynamic expansion to Coulomb explosion with decreasing frustration parameter $\alpha$ from 100 to 1 is observed. See text for details. Adapted from [79].

The signatures of a transition in the leading expansion mechanism appears to be less clear in the ion spectra. However, the important role of recombination for the final ion distribution in the hydrodynamic case can be traced in the left panel of Fig $2.19 \mathrm{c}$ by comparing dashed (no recombination) and solid (with recombination) lines.

As the frustration parameters of the xenon clusters irradiated with $90 \mathrm{eV}$ pulses [94] which have been displayed above in Fig $2.18 \mathrm{c}$ range from 40 to 1500 , the results of the study by Arbeiter and Fennel [79] would indicate a contribution from Coulomb explosion only for smaller cluster sizes.

Single shot scattering on single particles in the XUV: Single large clusters in the gas phase were imaged for the first time in single $90 \mathrm{eV}$ pulses at the FLASH free-electron laser [32]. The scattering patterns of individual clusters with radii of $150 \pm 40 \mathrm{~nm}$ revealed an over-proportional increase of the scattering signal at large angles for increasing pulse intensity from $8 \cdot 10^{12}$ to $4 \cdot 10^{13} \mathrm{~W} / \mathrm{cm}^{2}$ as displayed in Fig. $2.20 \mathrm{a}$. The data were modeled with Mie theory for a homogeneous sphere. A strong increase in the imaginary part of the refractive index was found, which is shown in Fig. 2.20 b. Such an increase in $\beta$ corresponds to a tenfold increase of the absorption in the cluster above the bulk-value of xenon. The observations were 


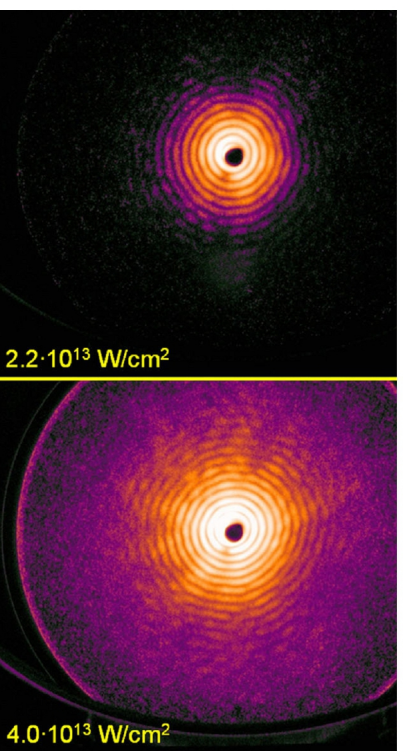

a)

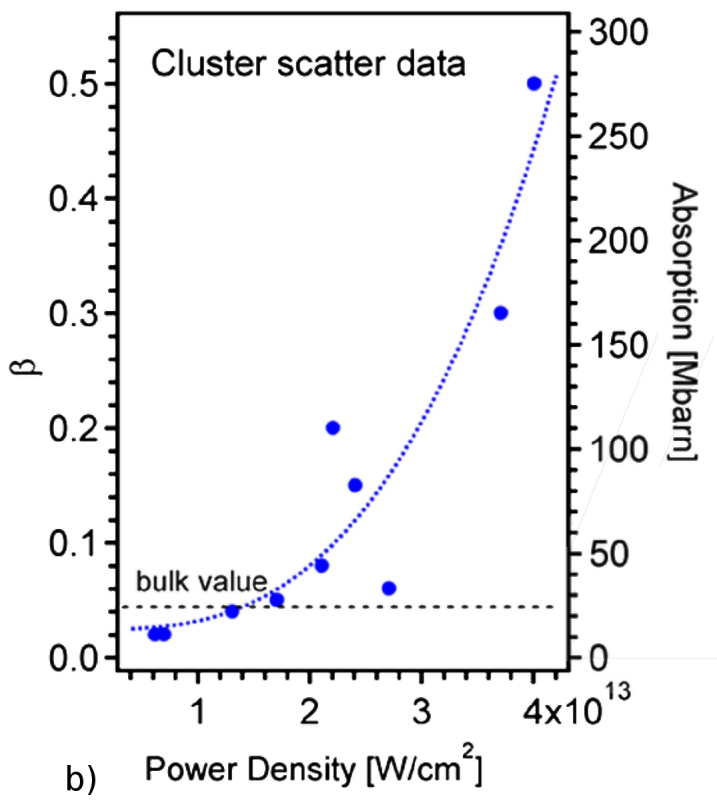

b) Power Density $\left[\mathrm{W} / \mathrm{cm}^{2}\right]$

Figure 2.20: Scattering experiments on individual xenon clusters at $90 \mathrm{eV}$ photon energy reveal an over-proportional increase of the scattering signal at large angles for increasing pulse intensity. By modeling the data with Mie's theory, the refractive index can be extracted from the scattering patterns, indicating a strong increase in the absorption related imaginary part $\beta$. Adapted from [32].

interpreted as a transiently high abundance of the resonant charge state $\mathrm{Xe}^{4+}$. These results will be further discussed in this work in section 4.5.

The finding of intensity dependent changes in the scattering signal shows the capability of single particle scattering experiments to gain insight into ultrafast plasma processes [32]. The dominant role of element specific electronic properties for the dynamics of clusters in intense short wavelength pulses, in particular in the vicinity of atomic resonances, manifests itself even more prominently in scattering experiments than in spectroscopy measurements of electrons and ions.

First results from the soft X-ray regime: With the recent upcoming of LCLS as the first free-electron laser reaching into the X-ray regime (see also section 3.1), first insight into $\mathrm{X}$-ray induced cluster dynamics could be gained. In the regime of $1 \mathrm{keV}$ excitation energy, inner shell electrons are mainly addressed by photo ionization processes. In atoms, shorter pulses with the same number of photons result in a decrease of energy absorption [29, 55]. The reason for this effect referred to as hollow atom is a competition between the Auger lifetime of the inner-shell vacancy and the pulse length. Only if the inner-shell vacancy has been refilled by an Auger decay, more photons can be absorbed. This transient transparency to X-ray photons was found to be further increased in clusters [30]. Due to efficient barrier suppression in the highly ionized cluster, the valence electrons become delocalized. This yields in a decreased overlap with the inner-shell states and subsequently longer Auger lifetimes $[30,105]$.

These findings might become important for future imaging of single molecules with atomic resolution. The constraints for the pulse duration before the structures are washed out were predicted to be limited by the lifetimes of inner-shell vacancies [27]. 


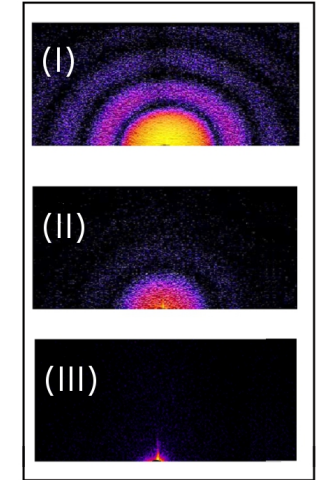

a)

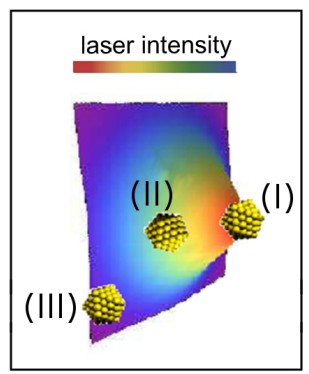

c)

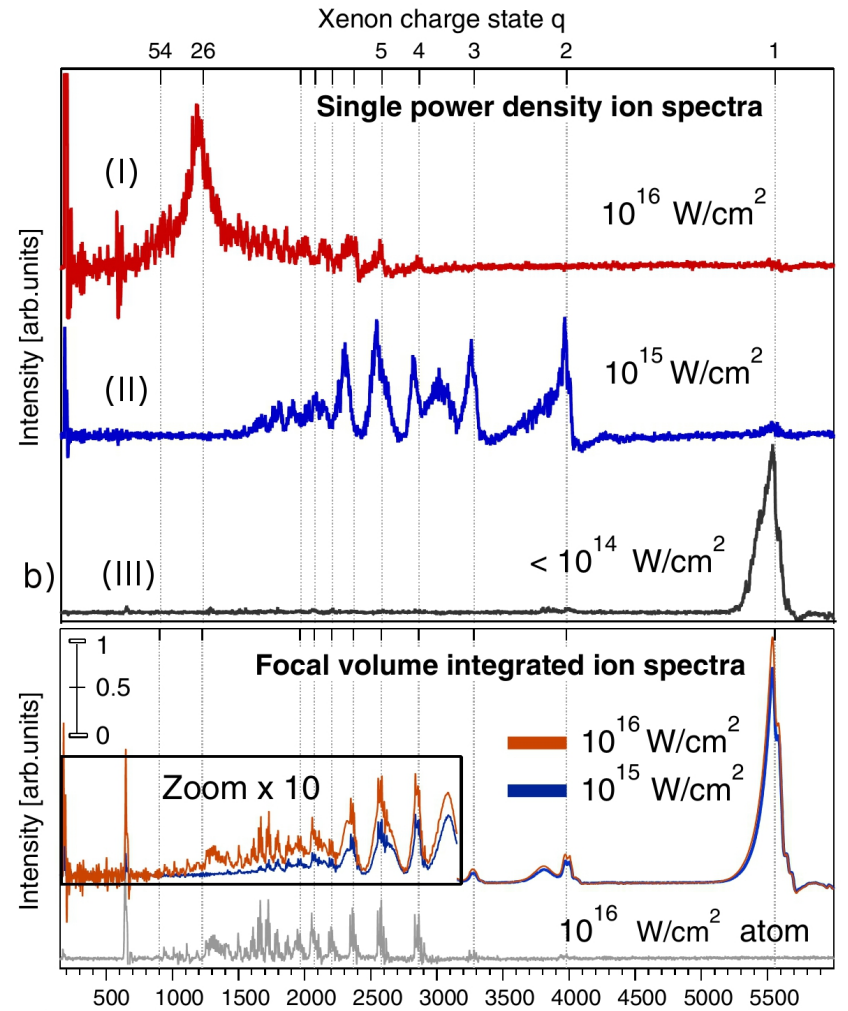

d) time-of-flight [ns]

Figure 2.21: a) The scattering patterns of single clusters in soft X-ray pulses are used to sort the data for cluster size and FEL intensity. Scattering patterns of three clusters with $R \approx 30 \mathrm{~nm}$ exposed to different FEL intensities are shown. b) The corresponding ion spectra differ radically. Spectrum (III), taken at $\approx 10^{14} \mathrm{~W} / \mathrm{cm}^{2}$ reveals only singly charged xenon ions. For two orders of magnitude more power density, the charge states in spectrum (I) peak around xenon 26+. Low charge states are virtually absent. c) The different intensities of cases (I), (II), and (III) correspond to different position of the respective clusters in the focus profile. d) The signatures are less clear in averaged data over ensembles of clusters in the focus profile. The spectra taken at different pulse energies comparable to the single shots above are completely dominated by lower charge states and show only small differences at higher charge states. In addition, comparison with an atomic spectrum hints towards a considerable contribution in the averaged cluster signal from the uncondensed part of the beam. [104]

By combining single shot scattering on single clusters with coincident spectroscopy measurements, a new method for studying ionization dynamics of clusters on perfectly defined systems was developed. The first coincident single cluster imaging and ion spectroscopy measurements on clusters were carried out at a photon energy of $800 \mathrm{eV}$ [104]. The interaction of single xenon clusters with the soft X-ray pulses was studied by means of ion spectroscopy, using the simultaneously taken scattering patterns to sort for cluster size and power density. The results are shown in Fig. 2.21. Three data sets are displayed consisting of the scattering patterns (Fig. 2.21 a) and the ion spectra (Fig. 2.21 b) of individual xenon clusters with $30 \mathrm{~nm}$ radius exposed to FEL intensities between $10^{14}$ and $10^{16} \mathrm{~W} / \mathrm{cm}^{2}$. The corresponding ion spectra differ radically. Spectrum (III) taken at $\approx 10^{14} \mathrm{~W} / \mathrm{cm}^{2}$ exhibits only singly charged xenon ions, while the charge states in spectrum (I) at $10^{16} \mathrm{~W} / \mathrm{cm}^{2}$ reveal a rather narrow distribution around xenon $26+$. Low charge states are virtually absent.

This finding can be understood as efficient suppression of recombination in the hot nanoplasma. 
The xenon atoms in the cluster become highly ionized in a sequence of single absorption steps from inner-shell electrons followed by subsequent Auger cascades. High excess energies result in high kinetic energies of the electrons. Even though less than $1 \%$ of the photo-activated electrons for case (I) become outer-ionized, the competition between cluster expansion and cooling of the electrons, i.e. decrease in ion density and increase in recombination probability is shifted towards a freeze-out of recombination processes.

In contrast, the averaged spectra shown in Fig. $2.21 \mathrm{~d}$, which contain the information from a large ensemble of clusters distributed over the focus profile reveal a by no means clear signature. The spectra were taken at an average focal intensity corresponding to the cases (I) and (II). Both are dominated by low charge states from the wings of the focal profile. Slight differences appear at high charge states, however, they can not be clearly distinguished from atomic signal from the uncondensed part of the cluster beam, as indicated by comparison with the atomic ion spectrum.

These findings emphasize the possibilities of coincident single shot scattering of single clusters and spectroscopy measurements to study light-induced dynamics in clusters under well defined experimental conditions [104]. The setup for simultaneous imaging and ion spectroscopy of single clusters at the FLASH free-electron laser which was developed in the framework of this thesis will be described in the next section. 


\section{Chapter 3}

\section{Experimental setup}

In this thesis, the interaction of large xenon clusters with high intense XUV pulses has been studied in a combined scattering and spectroscopy approach. The data have been obtained at the free-electron laser in Hamburg FLASH. This chapter will give a survey on the experimental basics. In section 3.1, the principles of light generation in free-electron lasers will be explained and important characteristics of FLASH are summarized. Some insight is given on optical layouts for guiding and focussing the XUV light.

In the second part of this chapter, the experimental setup for simultaneous scattering and ion spectroscopy of single clusters will be introduced. Crucial components of the experiments, such as the generation of clusters and the detection of scattered light and ions will be discussed in more detail.

\subsection{FLASH Free-electron laser for short wavelength radiation}

Free-electron lasers (FELs) hold great promise as high power, short pulse, coherent sources for short wavelength radiation $[106,14]$. The continuous progress in X-ray physics of the last century has been tightly coupled to the advancement of light sources. Since the discovery of $\mathrm{x}$ rays in 1895 by Wilhelm Conrad Röntgen [107] the brightness of X-ray sources has been increasing more than four orders of magnitude per decade in the last 50 years as shown in Fig. 3.1a [34]. This quantity, the brightness $B$, is defined as the number of photons $n$ divided by the product of the beam area $A$, the opening solid angle of the radiation beam $\theta$, the pulse length $t$ and the fractional bandwidth of energy $\triangle E / E$,

$$
B=\frac{n}{A \cdot \theta \cdot t \cdot \frac{\triangle E}{E}} .
$$

The increase in brightness (or peak brilliance) is illustrated in Fig. 3.1b, where the capabilities of modern synchrotron sources and short wavelength free-electron lasers are displayed. The short and bright pulses of FLASH and LCLS exceed the other sources by more than seven orders of magnitude $[108,16]$. A single FEL pulse of 10 to 100 fs duration typically contains $10^{12}-10^{13}$ photons, as many as the best synchrotron radiation beamlines deliver in about one second [109]. In the following paragraph the underlying physical processes and technical principles are briefly reviewed. A more detailed compendium can be found in the literature $[110,106,14]$. 

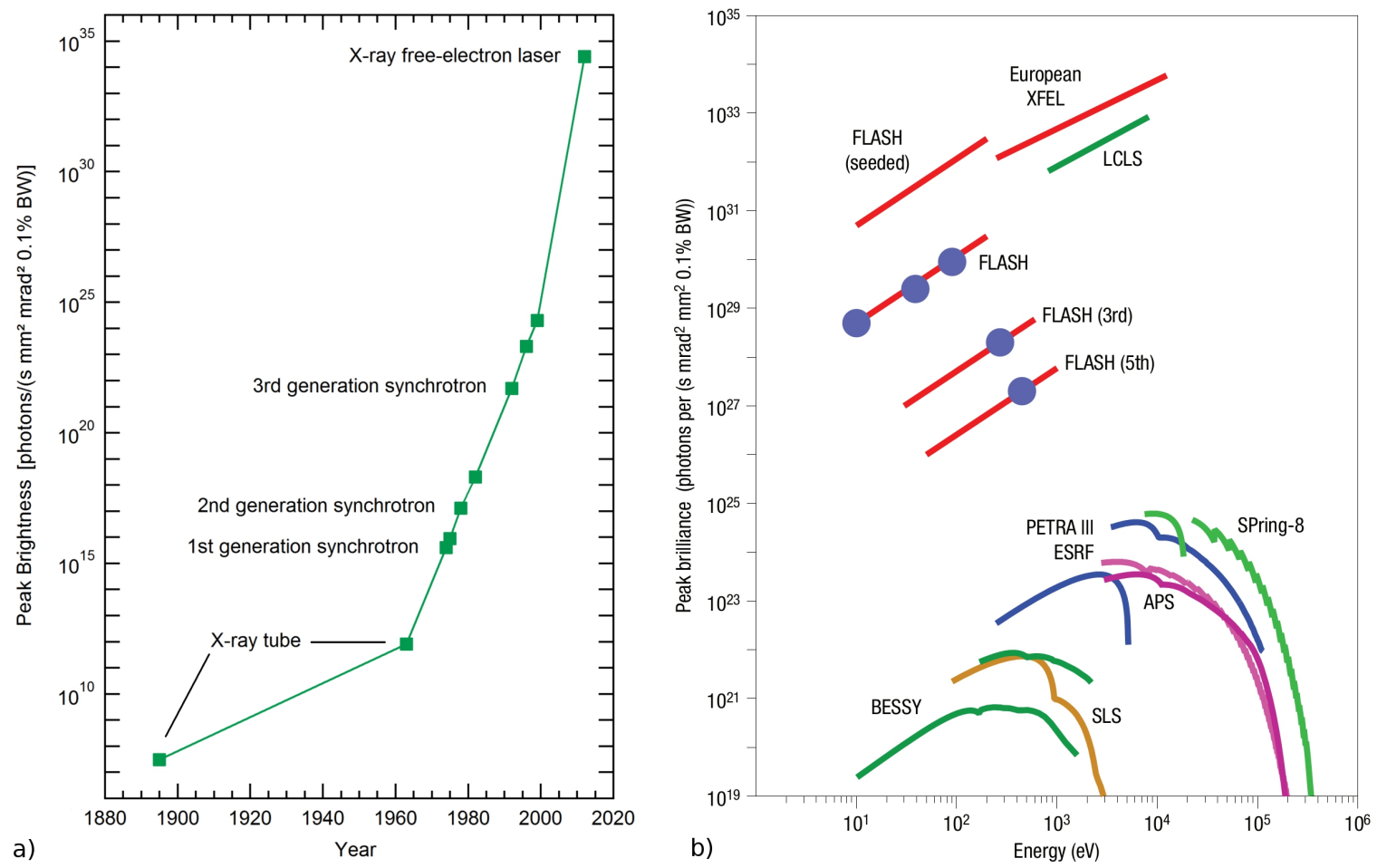

Figure 3.1: a) Development of peak brilliance of X-ray sources since 1895. Based on [34]. b) Peak brilliance of modern X-ray light sources. From [108].

\subsubsection{Basic principle of a free-electron laser}

Free-electron lasers and modern synchrotron sources share a common basic principle for the generation of light. Fig. 3.2 illustrates the evolution of synchrotron sources from a simple bending magnet (Fig. 3.2a) to the use of magnet arrays (Fig. 3.2b-d) [111]. Electrons are accelerated to relativistic kinetic energies and forced on a slalom course through a periodic stack of alternated dipole magnets. With every turn, the electrons emit light. In a so called wiggler device, the amplitude of the electron trajectory is rather large and consequently also the solid angle in which the photons are emitted. This leads to an incoherent superposition of the radiation and the emitted power rises linear with the number of magnet pairs within the wiggler. In contrast, the emitted power in an undulator rises quadratically with the number of magnet pairs. The periodic deflection of the electrons is so small that the radiated light from an electron in every undulator period can overlap and thus interfere. Constructive interference appears for a phase match between the electron energy and the undulator period, yielding a distinct resonant wavelength

$$
\lambda_{\text {res }}=\frac{\lambda_{u}}{2 \gamma^{2}}\left(1+\frac{K^{2}}{2}+\gamma^{2} \theta^{2}\right)
$$

with the undulator period $\lambda_{u}$, the Lorentz factor $\gamma=\left(1-v^{2} / c^{2}\right)^{-\frac{1}{2}}$, the undulator parameter $K$, and the observation angle $\theta$. The dimensionless parameter $K$ is a measure of the oscillation magnitude and is given by

$$
K=\frac{e B \lambda_{u}}{2 \pi m_{e} c}
$$



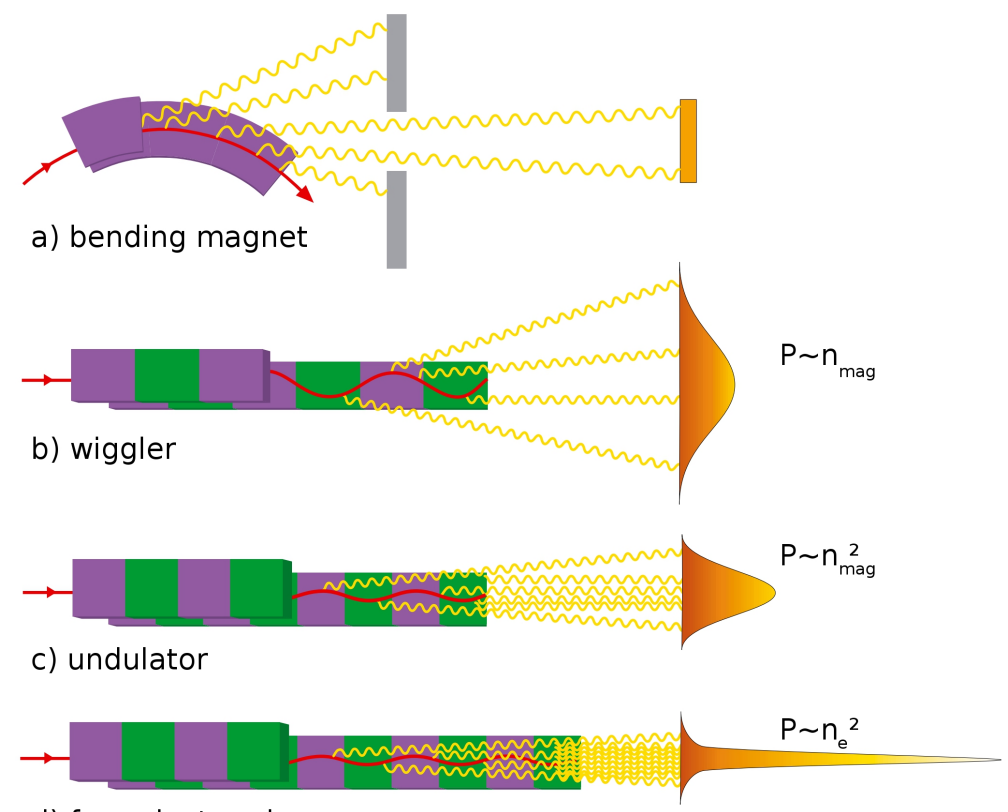

d) free-electron laser

Figure 3.2: Radiation of a bending magnet, a wiggler, an undulator and a free-electron lasers. $P$ is the irradiated power in forward direction. Adapted from [111].

with the magnetic field $B$ of the undulator. The $K$-parameter of a wiggler is $>10$, typical values for an undulator are in the order of 1 .

The radiation of free-electron lasers is also generated in undulators but the emitted power does not only increase quadratically with the number of undulator periods but also with the number of electrons involved in the lasing process. The resonant wavelength of a free-electron laser is given by an equation equivalent to the undulator equation 3.2 with $\theta=0^{\circ}$ :

$$
\lambda_{r e s, F E L}=\frac{\lambda_{u}}{2 \gamma^{2}}\left(1+\frac{K^{2}}{2}\right) .
$$

The electron energy matches the undulator period such that the light is overtaking the relativistic electrons by exactly one cycle while they move one undulator period further. This key process in free-electron laser physics displayed in Fig. 3.3 is referred to as slippage. The same phase relation is valid in an undulator for synchrotron radiation at $\theta=0^{\circ}$ but there the interference effects apply independently to the radiation probability for each electron, with no

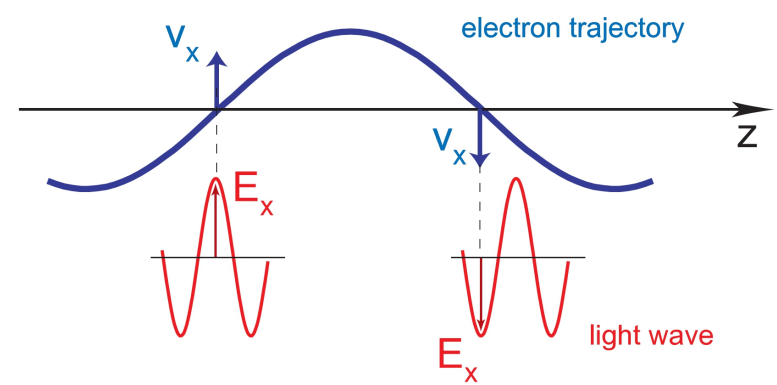

Figure 3.3: Slippage of the light wave of one optical wavelength per undulator period. From [112]. 
(a) Low gain FEL

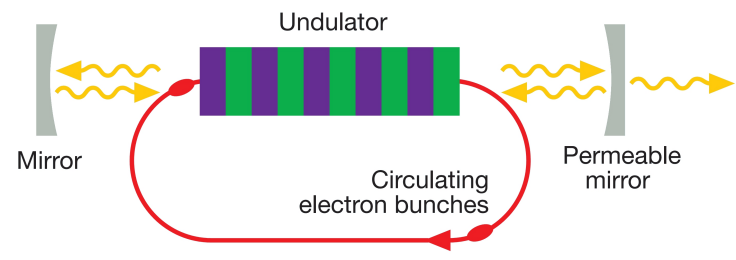

(b) SASE FEL

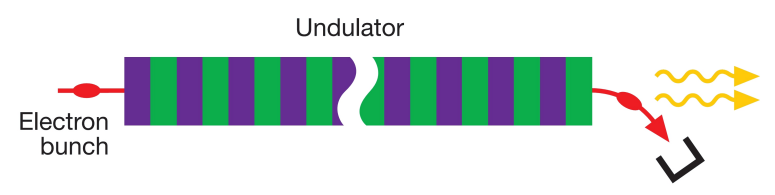

Figure 3.4: a) Principle of a low gain FEL working in the infrared or optical regime. b) Principle of a high gain single-pass FEL. From [109]

inter-electron effects. The electrons can never experience their own light field as their velocity is always $<c$. However, in a free-electron laser the electrons are extremely well compressed into a small bunch with a very sharp kinetic energy distribution. These characteristics, the spatial and the velocity distribution of the electron bunch, are gathered in a quantity called emittance. A low emittance of the electron bunch is the basic condition for the lasing process. In this case a present electromagnetic field can interact back on the electrons and force them to stimulated emission.

The requirements on the quality of the electron bunch are therefore especially high for short wavelength FELs. As illustrated in Fig. 3.4a, in the infrared and visible regime it is possible to run free-electron lasers in the low gain range. In this concept a train of relativistic bunches recirculate within a storage ring through an undulator placed in an optical cavity. A small gain in radiation power of a few percent per undulator passage is sufficient to allow output powers in the order of Gigawatts [109]. Even though the resonance wavelength of an undulator can be shifted into the X-ray range by just increasing the electron kinetic energy, no normal incidence optics with reflectivities $>90 \%$ exist for wavelengths below $100 \mathrm{~nm}$. Therefore, short wavelength FELs are built on the principle of a single-pass high gain FEL as displayed in Fig. 3.4b. Here, all radiation is generated in a single passage through a very long undulator. For accelerating the electrons linear accelerators are used as only they allow for preserving the low emittance of the electron bunches required for the lasing process.

As the electron bunch orbits through the undulator some of the electrons will be accelerated and take energy from the light field, others will be decelerated and contribute energy to the light field depending on their phase (cf. Fig. 3.3). Because of the slippage, the electrons experience the same light field in every phase of their oscillation, which makes the interaction very efficient. In the long undulator of a high gain FEL, the periodically modulated energy is then transformed into a density modulation, as the faster electrons catch up with the slower ones. This process will continue until the electrons in the bunch form a package of slices with a spacing of $\lambda_{\text {res }}$, so called microbunches (illustrated in Fig. 3.5). Microbunching will lead to an increase of the radiated power proportional to the number of microbunched electrons $n_{e}$ squared because the electrons separated by $\lambda_{\text {res }}$ radiate like a point charge $Q=n_{e} \cdot e$. The number of electrons contributing to the lasing process increases while the bunch passes the undulator, yielding an exponential growth of the emitted power with undulator length. In Fig. 3.5 the growth is shown as a function of gain length $L_{g}$, the undulator length in which the power rises by a factor of e. After several $L_{g}$, when the microbunches are fully developed, laser saturation sets in.

In IR and visible FELs a concept is widely used which is referred to as seeding. In this case, the electromagnetic field which is amplified by the electrons is inserted from an external source such as a seeding laser. Another approach which is especially convenient for short wavelengths 


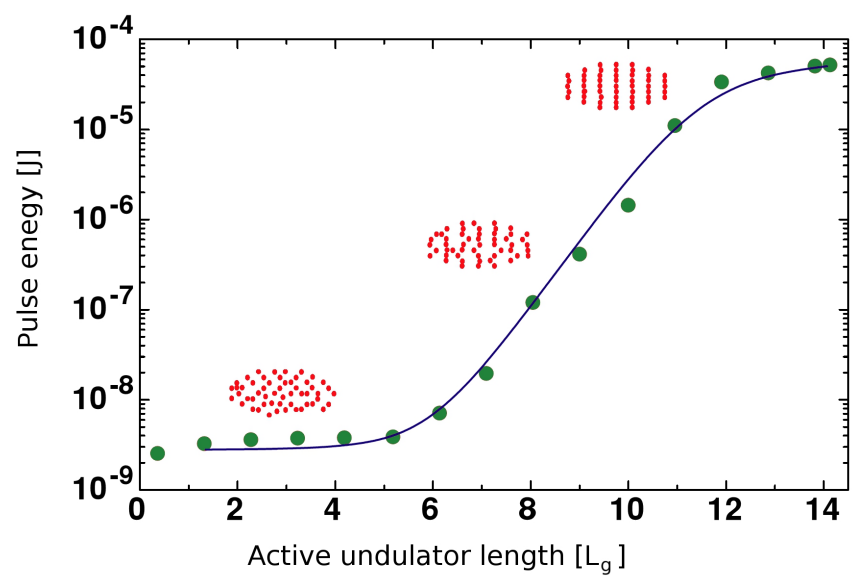

Figure 3.5: Gain length and microbunching. In a single-pass FEL the density modulation of the electron bunch develops along the travel through the undulator. The gain curve shows exponentially growth of the output power until optimal microbunching is reached and the pulse energy saturates. Adapted from [14] and [106].

where less laser sources exist is spontaneous undulator radiation being used for amplification. This process is referred to as SASE (self amplified spontaneous emission). SASE is used in most of the current short wavelength FELs, for example at FLASH $[108,16]$. Since the lasing starts from shot noise, the photon energy is subject to statistical fluctuations. SASE FEL radiation typically consists of more than one single pulse in time and frequency [113], even though SASE radiation also exhibits full transverse coherence [14]. As brighter pulses and smaller bandwidths can be achieved by seeding, concepts have also been developed for the short wavelength range. For example at FERMI, Trieste, a high-harmonic-generation (HHG) source is successfully used as seeding laser for an FEL in the VUV range [114]. At FLASH, there is also a seeding option under construction, using HHG [115]. Another approach for seeding short wavelength FELs is to monochromatize SASE radiation and use it as a seed beam in a second undulator $[116,117]$. Such self-seeding has been recently demonstrated for hard $\mathrm{x}$ rays at LCLS using a diamond crystal to extract a narrow-banded part of a SASE pulse [118].

\subsubsection{Characteristics of the FLASH FEL}

FLASH, a linac based facility at DESY has been the first short wavelength free-electron laser in operation $[120,15]$. Table 3.1 summarizes some important photon and pulse characteristics

\begin{tabular}{|l||c|c|}
\hline Parameter name & $\begin{array}{c}\text { Typical values } \\
2005-2009[119]\end{array}$ & $\begin{array}{c}\text { Parameters of our } \\
\text { experiment 2011 }\end{array}$ \\
\hline \hline Wavelength range (fundamental) & $6.5-47 \mathrm{~nm}$ & $13.6 \mathrm{~nm}$ \\
\hline Average single pulse energy & $10-100 \mu \mathrm{J}$ & $150 \mu \mathrm{J}$ \\
\hline Pulse duration & $10-70 \mathrm{fs}$ & $100 \mathrm{fs}$ \\
\hline Spectral bandwidth & $1 \%$ & $1 \%$ \\
\hline Photons per pulse & $10^{12}$ & $10^{13}$ \\
\hline
\end{tabular}

Table 3.1: FLASH pulse and photon characteristics 


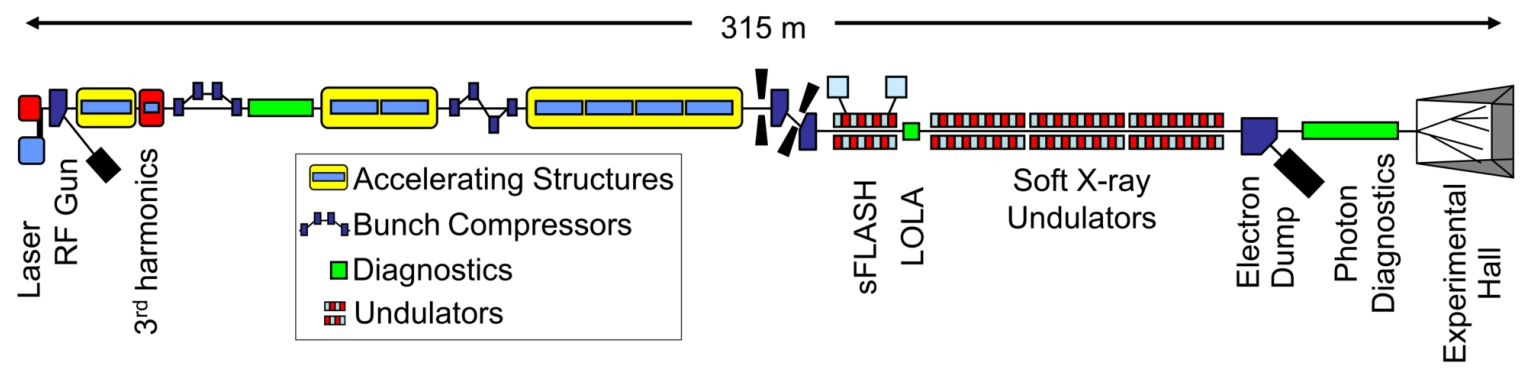

Figure 3.6: Layout of the FLASH facility, see text for description. Adapted from [119]

of FLASH. Typical parameters of the operation of FLASH from [119] are shown in the center column, the corresponding parameters used in the experiment for this work are displayed in the right column.

In Fig. 3.6, the current layout of FLASH $[109,119]$ is displayed. The electrons are produced in the RF gun, a laser driven photocathode placed in a radio-frequency cavity. From the gun the electrons are accelerated to a maximal kinetic energy of $1 \mathrm{GeV}$ in accelerator modules consisting of super-conducting niobium cavities. In between the accelerator modules the electron bunch is further compressed by inducing a chirp over the electrons and sending them through a bunch compressor, a small magnetic chicane. In addition Fig. 3.6 also contains the latest improvements and extensions such as an afterburner for enhancing the production of 3rd harmonic radiation and the sFLASH unit for HHG seeding.

The coherent radiation is produced in an undulator with a gain length of roughly $1 \mathrm{~m}$ and an overall length of $27 \mathrm{~m}$. The undulator period of $24 \mathrm{~mm}$ with a $12 \mathrm{~mm}$ gap between the magnetic poles yields a magnetic field $B$ of 0.5 T. Using Eq. 3.3 the $K$-parameter of the FLASH undulator is $K=1.1$. Assuming a maximal electron energy of $1 \mathrm{GeV}$, Eq. 3.4 yields a minimal wavelength of $5 \mathrm{~nm}$.

After the undulator, the electron bunch is extracted with a dipole magnet and sent into a beam dump. The light pulses emerge from the accelerator tunnel and enter the experimental hall. Several photon diagnostics are available at this stage, such as a spectrometer for wavelength determination and a gas monitor detector for online pulse energy measurements (for further details see [121]). The FLASH pulses can be guided into 5 different beamlines. Due to the strong absorption of soft X-ray radiation in any material, the beam is transported through a windowless UHV system. In the next paragraph brief insight is given in the possibilities of guiding and focussing the bright XUV light into the experiment.

\subsubsection{Guiding and focussing optics for XUV light}

Due to the lack of lens materials, which could be used for short wavelength radiation from VUV to soft X-ray, other approaches become necessary. Mainly two types of mirrors are used to guide and focus XUV radiation [35].

Gracing Incidence Mirrors are optics with a high transmission in an extended wavelength range. Plane carbon mirrors are for example used at FLASH to deflect the FEL beam into the different beamlines [122].

The basic principle of these mirrors corresponds to total internal reflection, widely used in the visible range for example in fiber optics. In the VUV to soft X-ray range, the real part of the refractive index of materials is typically slightly smaller than one $\left(n^{\prime}<1\right)$. As total reflection always occurs from the optically thin medium, in the XUV total external reflection 

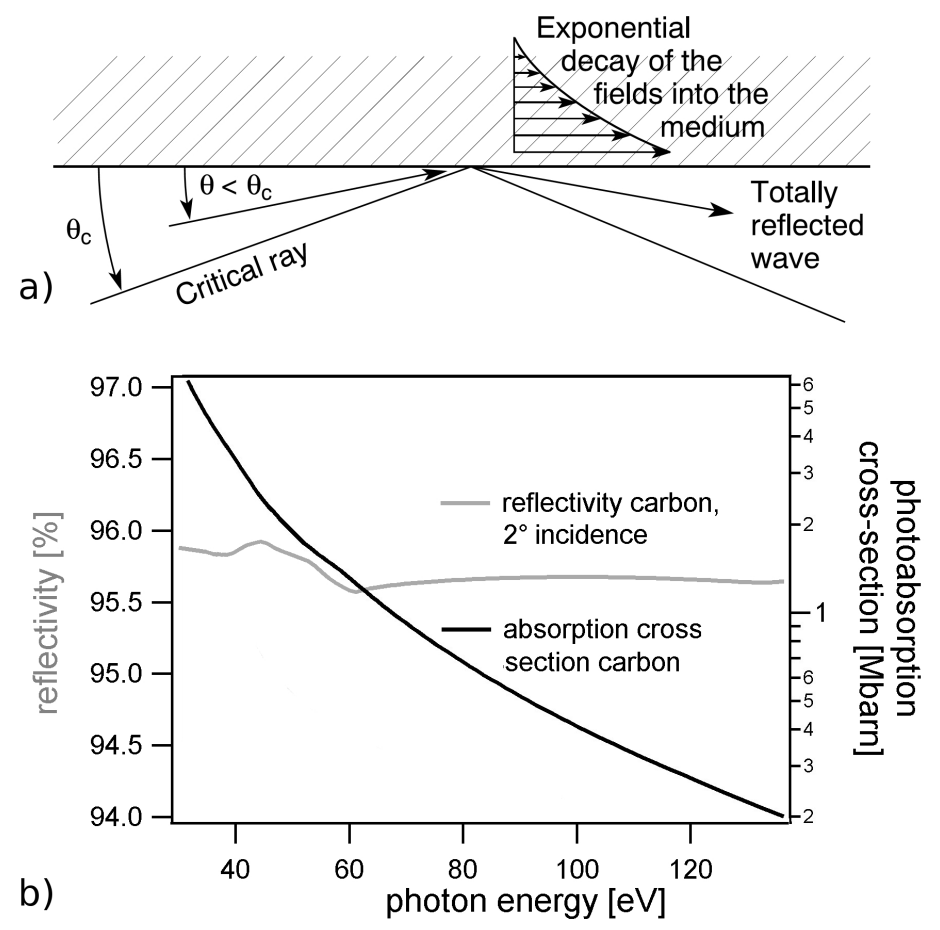

Figure 3.7: a) Principle of total external reflection of XUV radiation. From [35] b) Reflectivity of a carbon surface under $2^{\circ}$ and photo absorption cross-section from 30 to $150 \mathrm{eV}$. Data from Henke tables [60].

under gracing incidence can be used. The mechanism is sketched in Fig. 3.7 a. Without any absorption, optimal reflectivity is yielded at the critical angle $\theta_{\text {crit }}=\sqrt{2 \delta}$. However, an evanescent wave does penetrate the material up to a certain depth. Therefore, a finite absorption $\beta>0$ results in an optimal reflectivity for incident angles smaller than $\theta_{\text {crit }}$.

The surface material has to be selected according to the designated photon energy region. In the range relevant for FLASH, carbon coated mirrors are chosen due to their high reflectivity and flat absorption cross-section [123]. In Fig. $3.7 \mathrm{~b}$ the reflectivity of a carbon mirror under $2^{\circ}$ gracing incidence and the atomic photo absorption are displayed. The data is taken from the Henke tables [60].

Based on the principle of total external reflection, focussing optics can be manufactured as well. Carbon coated ellipsoidal mirrors with a focal distance of $2 \mathrm{~m}$ are installed at beamlines BL2 and BL3 producing a focus with $20-30 \mu \mathrm{m}$ diameter (FWHM) [121].

Multilayer mirrors are the second approach for reflecting short wavelength light. Fig. 3.8 a demonstrates that multilayer mirrors bridge the gap of (near-)normal incidence mirrors in the VUV to soft X-ray range [35].

The reflectivity at normal incidence of a material with refractive index $n=1-\delta+i \beta$ can be obtained by the Fresnel equations [42] to

$$
R \simeq \frac{\delta^{2}+\beta^{2}}{4} \ll 1 .
$$

Therefore, the normal incidence reflectivity on a surface will go down for higher wavelengths, where only small deviations of $n$ from unity occur. However, a higher reflectivity can be achieved again by constructive interference of the reflection on many layers. In hard X-ray regime natural crystals can be used which exhibit atomic layer distances in the range of the wavelength. In XUV multilayer mirrors can be produced. 

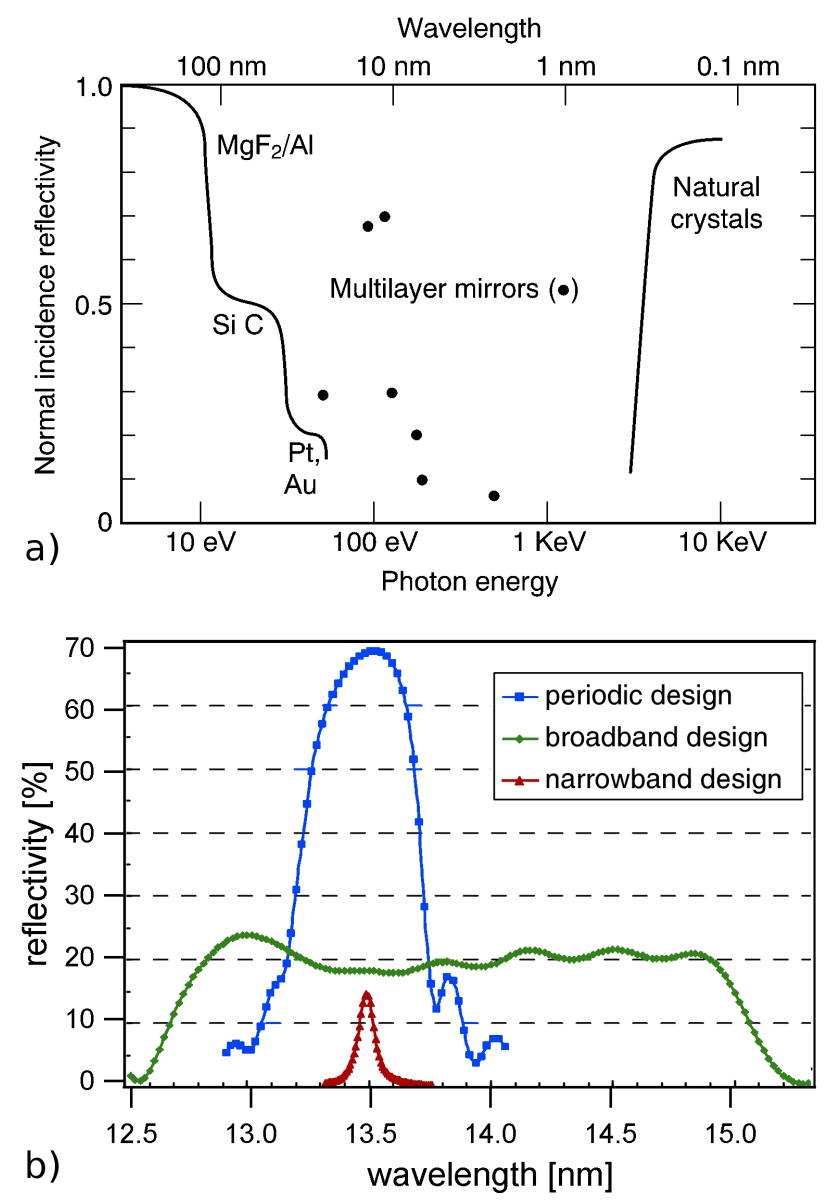

Figure 3.8: a) Possibility to produce near normal incidence mirrors from visible to X-ray regime. Multilayer mirrors close the gap in the XUV. From [35] b) Measured reflectivity of MoSi multilayer mirrors optimized for $13.5 \mathrm{~nm}$. Adapted from [124].

With sputtering techniques thin layers of two materials with different optical properties are deposited alternately on ultrasmooth substrates. The materials are chosen such that for both the absorption is low while they exhibit a great difference in the reflectivity, i.e., the real part of the refractive index. Incoming light propagates into the mirror through many layers. At every layer transition some light is reflected. For a distinct wavelength matching the modulation period, the reflected rays interfere constructively, producing an overall high reflectivity. For a photon energy of $91 \mathrm{eV}$ or $13.6 \mathrm{~nm}$ wavelength respectively, highly reflecting MoSi multilayer mirrors with close to $70 \%$ reflectivity can be manufactured [124] as shown by the curve for periodic design in Fig. $3.8 \mathrm{~b}$.

The reflectivity of multilayers is limited by the finite absorption of materials in the XUV and by the interface roughness between the layers. It is therefore lower than for gracing incidence mirrors. Also only a rather narrow bandwidth is reflected. On the other hand, multilayer mirrors can be optimized to reflect either in (near-)normal incidence or for another arbitrary angle. This makes them favorable for many otherwise space consuming optical layouts. A number of experiments carried out in our group [32, 24, 125, 126] were based on a back reflecting geometry using a multilayer mirror with a short focal distance. It could be mounted within an experimental setup and produced a smaller focal spot and thus higher power densities than a farer distant beamline optic. The procedure of optimizing and characterizing the focal density will be described in section 3.2.4. 

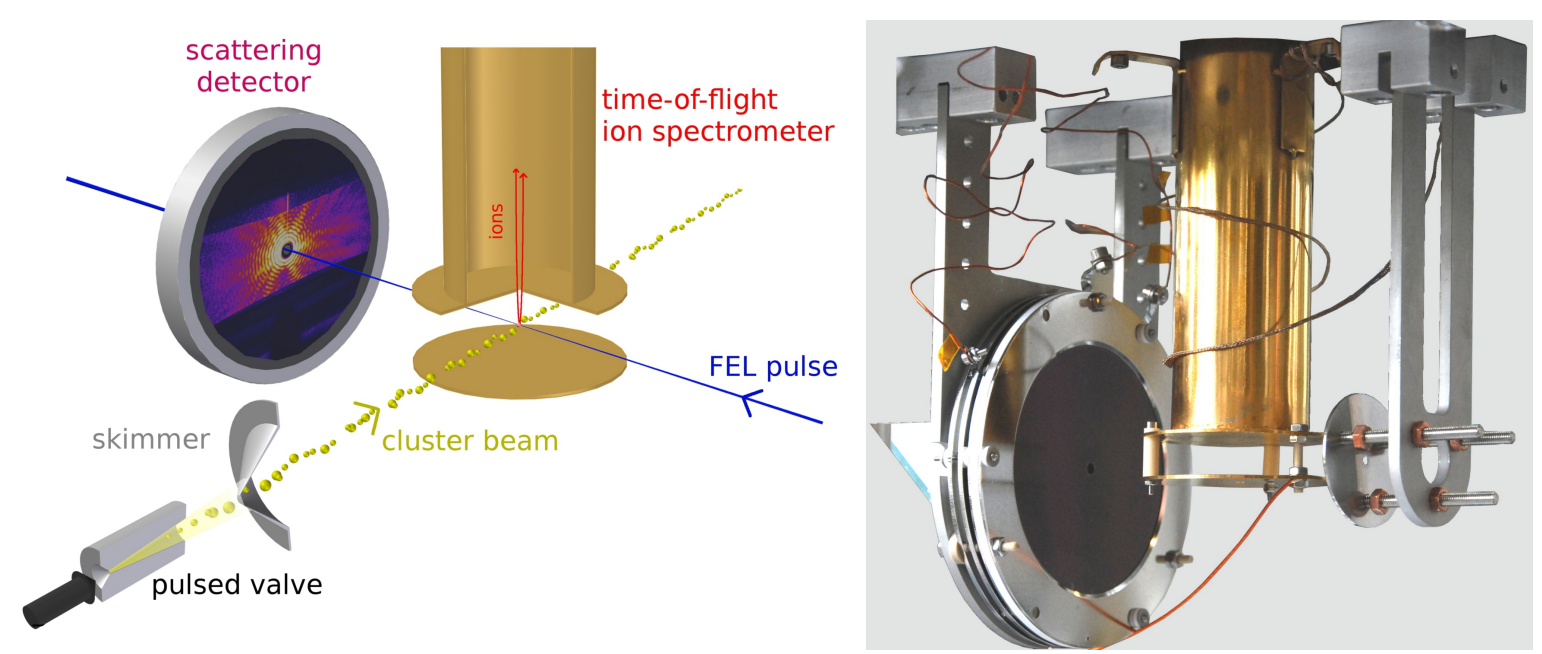

Figure 3.9: Left: Setup scheme of the experiment for coincident imaging and ion spectroscopy of single clusters at FLASH. Right: Photo of the detectors assembled around the interaction region

\subsection{Experiment for imaging and ion spectroscopy of single clusters}

In section 3.1 the generation and handling of highly intense short pulses from the FLASH FEL have been introduced. This brilliant light source enables a new kind of experiments where individual particles can be studied in single pulses. In this section, the experimental setup developed for imaging and ion spectroscopy of single large clusters is described. Experimental principles and methods are laid out in more detail in the subsequent paragraphs.

\subsubsection{Experimental layout}

The setup for simultaneous imaging and ion spectroscopy of single clusters at FLASH is displayed in Fig. 3.9. The experiment was carried out in July 2011 in the beamline focus of BL2, using $100 \mathrm{fs}$ pulses with up to $10^{13}$ photons of $91 \mathrm{eV}$ photon energy.

A cluster beam was generated by supersonic expansion of xenon gas through a cryogenically cooled, pulsed nozzle. The clusters were guided into the main chamber through a set of differentially pumped conical skimmers. Details on the cluster generation are discussed in section 3.2.2. The cluster beam was further skimmed down by a movable piezoelectric driven skimmer slit with an adjustable width between 0 and $1.5 \mathrm{~mm}$ (cf. [127], not displayed in Fig. 3.9). Thus, the measurements could be done in "single cluster mode" at a target density with less than one cluster at a time in the focal volume.

The cluster pulse was spatially and temporally overlapping with the focussed FEL pulse. In a combined imaging and spectroscopy approach, two different kinds of products of the interaction were measured shot-to-shot, positively charged fragments of the clusters and elastically scattered light. The scattering patterns were detected by an MCP / phosphor screen stack as described in section 3.2.3. The visible image on the phosphor screen was coupled out via a $45^{\circ}$ mirror and recorded with an out-of-vacuum CCD camera. The FEL beam could exit the interaction region through a center hole in all components of the scattering detector. For the ion measurement the "bipolar tof" was used, as discussed in section 3.2.4. 

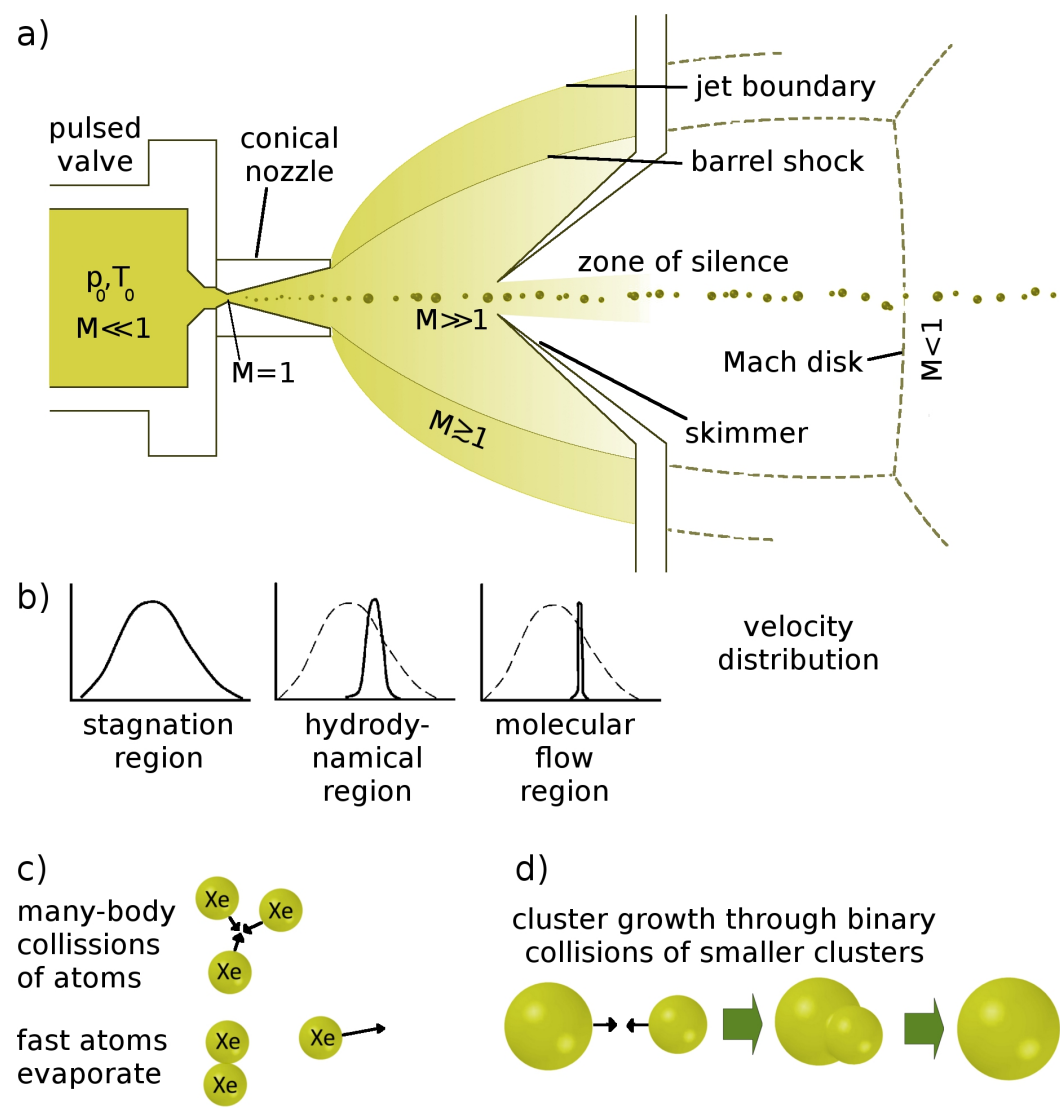

d)

cluster growth through binary collisions of smaller clusters

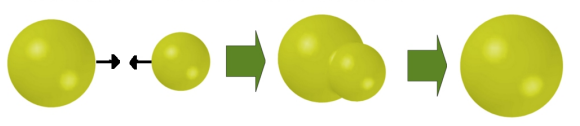

Figure 3.10: a) Schematics of a supersonic gas expansion through a conical nozzle. Shock waves surround the zone of silence in which clusters can grow. A skimmer guides the central part of the beam in a differentially pumped section before the Mach disk destroys the clusters. b) Development of the particle velocity distribution in a supersonic gas expansion (adapted from [130]). c) Mechanisms of cluster growth. At first, dimers are built through 3-body collisions. As long as the ratio of atoms to clusters is large, they grow through monomer addition. d) Later, cluster-cluster aggregation becomes the dominant process.

\subsubsection{Cluster generation}

The rare gas clusters are produced in a supersonic gas expansion through a nozzle behind a pulsed valve. In this section a brief overview of the physics of supersonic jets is provided and the emergence of clusters in supersaturated gas is described. Also, the mechanisms of cluster growth are explained and hence, scaling laws for cluster size distributions are presented. The temporal profile of cluster jets due to nozzle operation in pulsed mode is discussed. Finally, typical parameters of this experiment are summarized.

Conditions in a free jet: Free jets are widely used for the generation of cold atomic and molecular beams and also serve as a source for cluster beams [128, 129]. A free jet produced by supersonic expansion through a nozzle is schematically illustrated in Fig. 3.10a. Gas expands from a stagnation chamber with an initial pressure $p_{0}$ and a temperature $T_{0}$ through a small orifice with diameter $d$ into vacuum. The expansion exhibits three stages, the stagnation region, the hydrodynamic region and the molecular flow region [130]. The characteristic velocity distributions of these stages are displayed in Fig. 3.10b. In a supersonic 
expansion the random velocity of the atoms in the stagnation region is transformed into onedimensional directed motion with a narrow relative velocity distribution. This is equivalent to a strong cooling of the gas. Equalization of the velocities takes place through collisions between the atoms. While the one dimensional flow velocity is rising, the particle density and thus the collision frequency decreases with the increasing distance to the nozzle throat. In particular, the number density of particles can be expected to decrease with the inverse square of distance, as the streamlines of the expanding gas resemble those of a point source flow [128]. At some point the jet leaves the collision dominated regime and enters the collision free molecular flow region, thereby reaching its terminal velocity. The maximum reachable flow velocity is given by

$$
v_{\infty}=1.581 \sqrt{\frac{2 k T_{0}}{m}}=204 \sqrt{\frac{T_{0} / K}{m / a m u}} \frac{m}{s},
$$

with $m$ being the atomic mass of the atoms.

Mach number: However, under realistic experimental conditions, the finite background pressure $p_{\text {back }}$ in the expansion chamber limits the free expansion to a system of shock waves as sketched in Fig. 3.10a. The shock waves reflected by the background pressure at the sides of the expansion cone are referred to as barrel shocks and the region where the velocity changes from supersonic to subsonic within a short distance in axial direction is known as Mach disk. Within these boundaries of the expansion, in the zone of silence, the adiabatic expansion takes place. A measure for the development of the flow velocity is the Mach number $M$, the ratio of the gas flow velocity to the local sound speed. If $M>1$ the particles move faster than information transport in the gas flow. Within the stagnation chamber $M$ is much smaller than 1 while at the nozzle throat, $M$ equals unity and then increases further in the hydrodynamic region with rising distance to the nozzle throat. In the boundary regions $M$ drops again to below 1 .

The position of the Mach disk from the nozzle throat is given by

$$
\frac{x_{\text {Mach }}}{d}=0.65 \sqrt{\frac{p_{0}}{p_{b a c k}}} .
$$

When reaching the Mach disk, the cold beam of atoms, molecules or clusters will be disturbed and reheated. Therefore, the Mach disk has to be located after the interaction region of the experiment. A practical possibility to shift the Mach disk away from the nozzle is to decrease $p_{\text {back }}$ by using differential pumping stages (cf. Eq. 3.7). The central, i.e. coldest part of the flow is cut out and transported into a differentially pumped chamber with a lower background pressure through a skimmer with an optimized shape and diameter in order to not disturb the gas flow (cf. Fig. 3.10a).

Cluster formation: The formation of clusters in a free jet can be explained as a phase transition from gaseous into liquid or solid state. Whether clusters emerge in this expansion process or not depends on the final pressure and temperature of the flow and in particular, if those parameters have left the gaseous domain. Fig. 3.11 illustrates the phase diagram of a supersonic expansion using the example of the vapor pressure curve of xenon (data from [131]). Typical stagnation conditions as used in this experiment $\left(p_{0}=8 \mathrm{bar}, T_{0}=240 \mathrm{~K}\right)$ are represented by point A. From there, the expanding gas follows the adiabatic line down to point $\mathrm{B}$, where it meets the vapor pressure curve $p_{v}(T)$. Under equilibrium conditions the subsequent expansion would follow the vapor pressure curve. Instead, it proceeds along the 


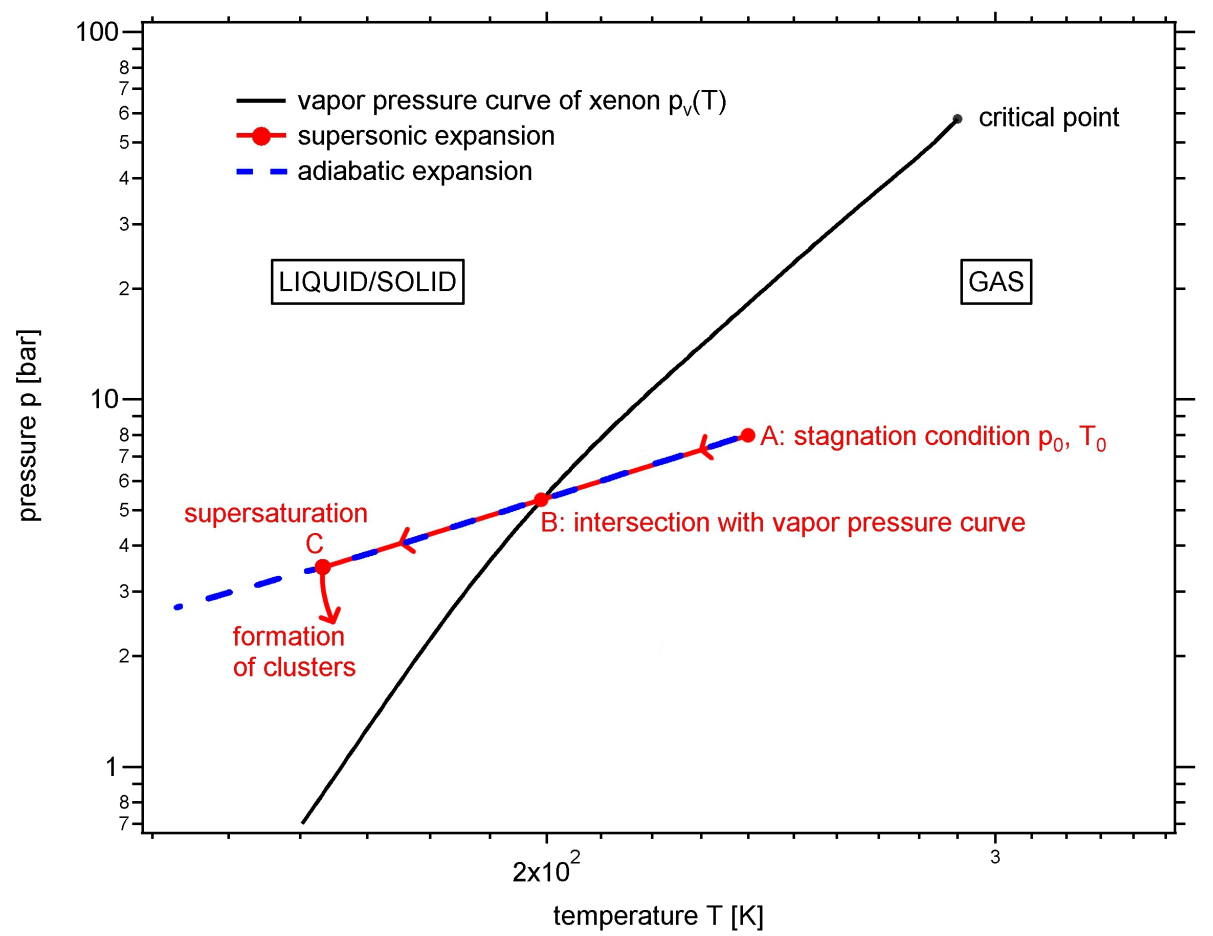

Figure 3.11: Vapor pressure curve of xenon. The path of a supersonic expansion with cluster condensation is sketched in red. The data has been taken from [131], the expansion path is adapted from $[128,130]$.

adiabatic line into a regime of supersaturation. At point $(\mathrm{C})$, condensation and formation of clusters sets in. At the same time the expansion departs from the adiabatic line and returns to the vapor pressure curve $[128,130]$.

As schematically illustrated in Fig. $3.10 \mathrm{c}$ the condensation process starts with the appearance of dimers formed in three-body collisions. Two atoms stick together, the third atom evaporates, taking away excess momentum and energy. When dimers are formed, they can act as condensation nuclei for further cluster growth. As long as the ratio of atoms to clusters in the jet is high the clusters grow through monomer addition. With rising numbers of clusters, coagulation, i.e. aggregation of smaller clusters will become the dominant process. Cluster growth through coagulation yields log-normal distributions of cluster sizes, whereas clusters generated from monomer addition form a size distribution with an exponential decay [132]. Typical cluster size distributions showing both of those features are displayed in Fig. 3.12.

Scaling laws for the average cluster size: The average final cluster size obtained with a distinct set of expansion parameters can be estimated using the empiric scaling laws developed by Hagena et. al [33]. For the same gas species, different combinations of the expansion parameters may lead to the same cluster size distribution. This relationship is expressed by the scaling parameter $\Gamma$ as a measure for condensation [128],

$$
\Gamma=n_{0} d_{e q}^{q} T_{0}^{0.25 q-1.5} .
$$

The numerical value of the exponent $q$ has been experimentally determined to be 0.85 . $n_{0}$ denotes the number density, $T_{0}$ is the gas temperature. The equivalent nozzle diameter $d_{e q}$ has to be used in case of a conical nozzle shape. For conical nozzles, the streamlines of the expansion are limited to the cone angle $2 \theta$ but remain otherwise unchanged. As the expansion 


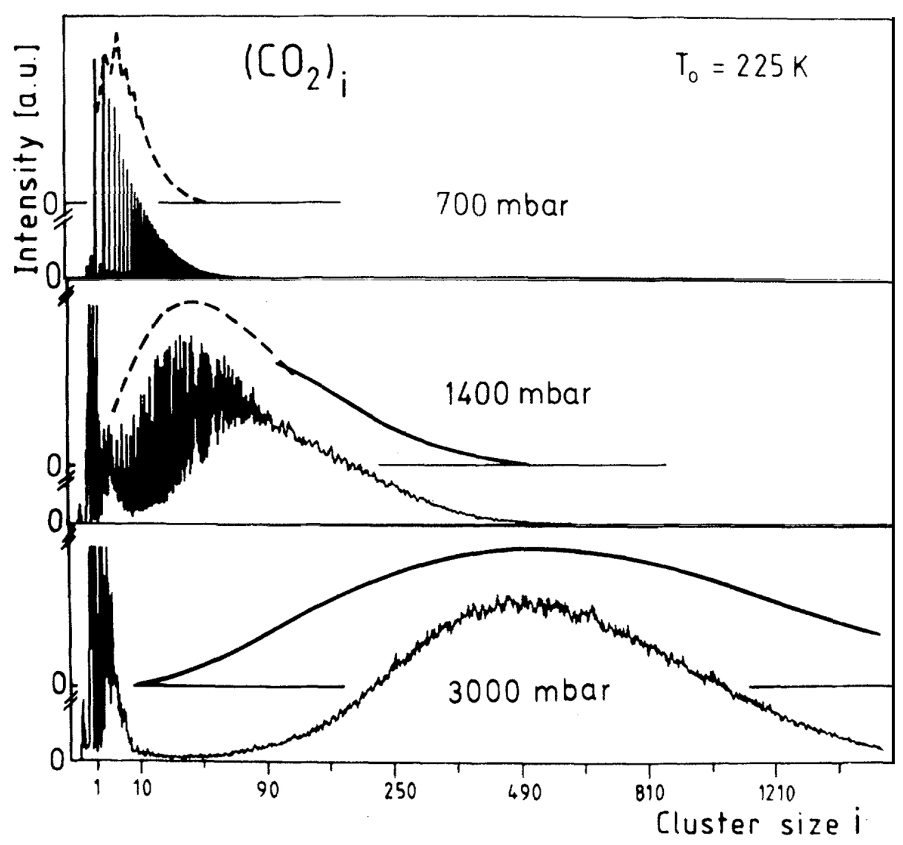

Figure 3.12: Cluster size distributions for a supersonic expansion of $\mathrm{CO}_{2}$ gas show the transition from monomer addition to cluster aggregation. The exponential decay at small cluster sizes is attributed to cluster growth through monomer addition, the lognormal size distribution developing for higher expansion pressures indicates coagulation of clusters. From [132].

cone of a simple orifice becomes less divergent for increasing nozzle diameter, the streamlines of a conical nozzle with diameter d mimic those of a much larger orifice. Thus, conical nozzles can reach the same $\Gamma$-value at a much lower gas load than simple orifices. This is described by the equivalent nozzle diameter $d_{e q}=d \cdot 0.74(\tan \theta)^{-1}$.

In order to connect the scaling parameters for the condensation of different kinds of gases, similarity laws can be introduced. In short, all material specific properties of gas species are gathered in a factor $K$, for example $K=5554$ in the case of xenon. A reduced scaling parameter $\Gamma^{*}$ can then be expressed as

$$
\Gamma^{*}=K \frac{p_{0} d_{e q}^{0.85}}{T_{0}^{2.29}},
$$

with $p_{0}$ in mbar, $d_{e q}$ in $\mu \mathrm{m}$ and $T_{0}$ in $\mathrm{K}$.

Consequently, the average number of atoms in a cluster results as a function of $\Gamma^{*}$ described by Hagena's scaling laws for different expansion regimes [133]:

$$
\begin{aligned}
\Gamma^{*}<350 & \text { no condensation } \\
350 \leq \Gamma^{*}<1800 & <N>=38.4\left(\frac{\Gamma^{*}}{1000}\right)^{1.64} \\
\Gamma^{*} \geq 1800 & <N>=33\left(\frac{\Gamma^{*}}{1000}\right)^{2.35} .
\end{aligned}
$$

For completeness it is mentioned, that for the regime of $\Gamma^{*}$ between $10^{5}$ and $10^{6}$ a deviation from Hagena's law was proposed [134],

$$
<N>=100\left(\frac{\Gamma^{*}}{1000}\right)^{1.8} .
$$

The cluster sizes in this experiment reach and exceed this regime. The obtained size distributions will be discussed in section 4.3 . 


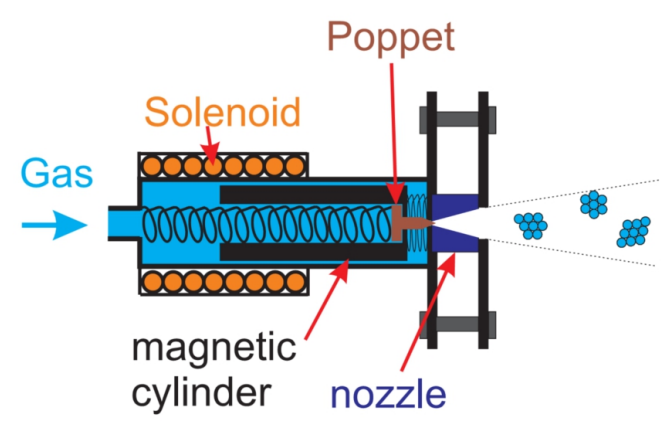

a)

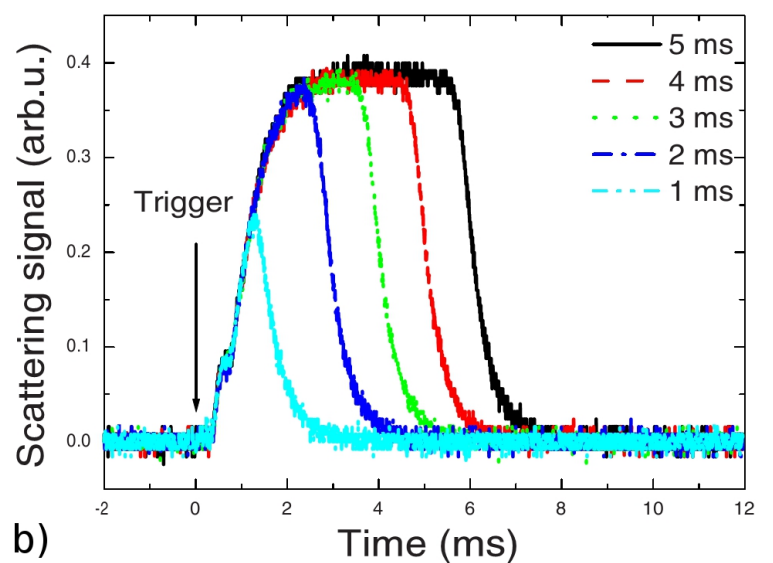

b)
Time (ms)

Figure 3.13: a) Function principle of the used solenoid valve (Parker 99 series [135]). Taken from [105]. Gas with a certain pressure and temperature fills the stagnation chamber. A magnetic cylinder with a sealing poppet is pressed by a spring into the nozzle throat. The solenoid pulls the cylinder with the poppet back, following a pulse trigger. Gas expands through the nozzle and may condense to clusters. b) Time resolved Rayleigh scattering signal for different valve opening times [136]. The formation of a plateau was observed only for opening times longer than $2 \mathrm{~ms}$.

\begin{tabular}{|l|l|}
\hline Parameter name & Typical values of our experiment \\
\hline Nozzle $d, \theta, d_{e q}$ & $200 \mu \mathrm{m}, 4^{\circ}, 2 \mathrm{~mm}$ \\
\hline Gas & xenon \\
\hline$p_{0}$ & $8 \mathrm{bar}$ \\
\hline$T_{0}$ & $220 \mathrm{~K}$ \\
\hline$p_{\text {back }}$ in expansion chamber & $\approx 5 \cdot 10^{-3} \mathrm{mbar}$ \\
$p_{\text {back }}$ in diff. pump. stage & $\approx 5 \cdot 10^{-5} \mathrm{mbar}$ \\
$p_{\text {back }}$ in interaction chamber & $\approx 5 \cdot 10^{-7} \mathrm{mbar}$ \\
\hline First skimmer (upstream) & $\approx 50^{\circ}$ cone, $0.5 \mathrm{~mm}$ diameter \\
Second skimmer (downstream) & $\approx 50^{\circ}$ cone, $1 \mathrm{~mm}$ diameter \\
\hline Valve opening time & $3-8 \mathrm{~ms}$ \\
\hline
\end{tabular}

Table 3.2: Typical parameters used for cluster generation.

Cluster parameters of the experiment: Typical values of the parameter sets used in this experiment are summarized in table 3.2. Such extreme expansion parameters are only possible in pulsed operation of the cluster beam because of the otherwise high gas load. FLASH light pulses as short as $100 \mathrm{fs}$ are used at a repetition rate of $5 \mathrm{~Hz}$. Thus, a great amount of pump load can be saved by opening the nozzle only 5 times per second using a pulsed valve. A solenoid driven valve (Parker 99 series [135]) as illustrated in Fig. 3.13 a is mounted on a cryostat $[105,137]$.

To ensure stable expansion conditions, the opening time of the valve was typically set to $3 \mathrm{~ms}$ in our experiments. This is in good agreement with Rayleigh scattering measurements with the same valve type displayed in Fig. $3.13 \mathrm{~b}$ [136]. There, a plateau was only found for valve opening times of $3 \mathrm{~ms}$ or longer. However, the cluster beam conditions changing with the timing of the light pulse in respect to the opening of the nozzle valve have been investigated in more detail in this work, as presented in section 4.3.2. The analysis of the scattering measurements for different valve trigger delays revealed a sharper rise of the cluster pulse (cf. Fig. $4.11 \mathrm{a}$ ) with a formation of a plateau within less than $1 \mathrm{~ms}$. 

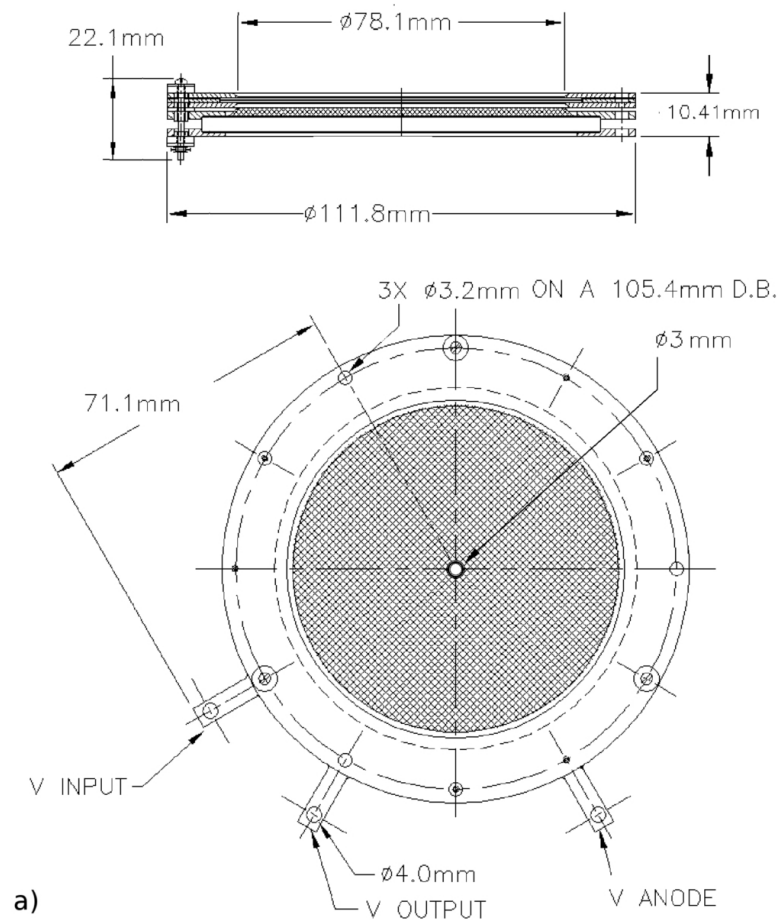

b)
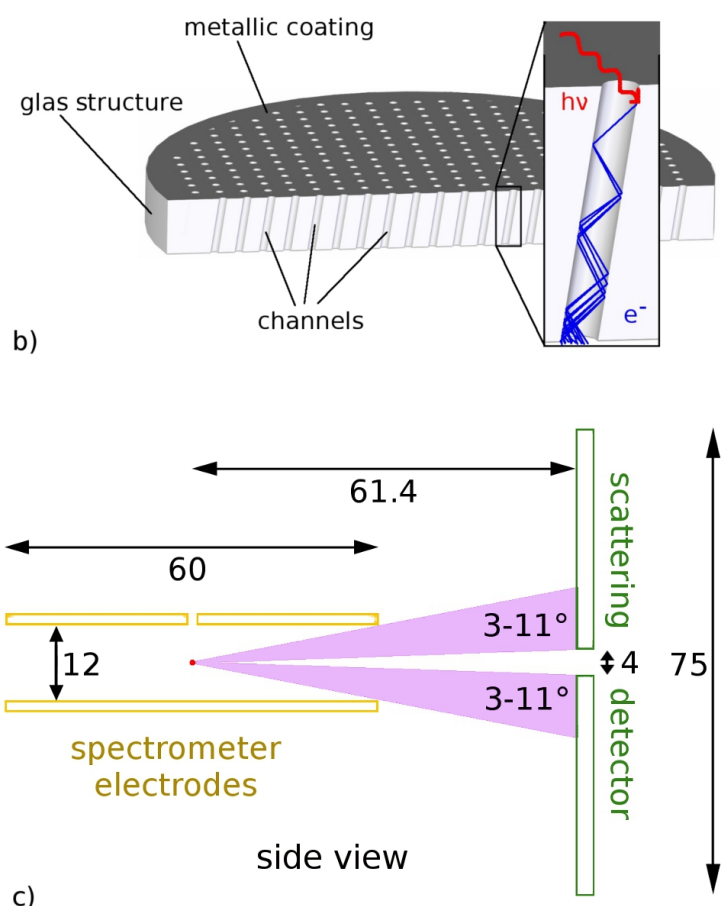

Figure 3.14: Scattering detector a) Large area MCP-phosphor stack with center hole. From [138] b) Function principle of a multichannel plate for photon detection. An incoming photon ejects electrons from the channel wall. The signal is amplified in a cascade of secondary ionization processes through the channel. c) Side view of the detector geometry. The detection angle is limited to $11^{\circ}$ by the shadow from the ion spectrometer electrodes. In vertical direction the diameter of the scattering detector of $75 \mathrm{~mm}$ defines the maximal scattering angle of $31^{\circ}$.

\subsubsection{Detection of scattered light}

The detection system for elastically scattered photons is the key component of the experiment. The position resolving area detector consists of a large-area multichannel plate (MCP) and a phosphor screen. Both have a $3 \mathrm{~mm}$ center hole for the exiting beam. A sketch of the detector is shown in Fig. 3.14a [138].

Multichannel plate: The working principle of a multichannel plate is sketched in Fig. 3.14b. An MCP can be understood as an array of miniature electron multipliers [139]. A plate with small, parallel channels is made from lead glass and coated with a material optimized for secondary electron emission (p.e. CsI). Between front and back of the plate, which are metal coated for electrical contact, a potential in the order of $1000 \mathrm{~V}$ is applied. Incoming photons result in the emission of electrons from the channel walls which are multiplied along the channel. The channels of this specific detector have a diameter of $25 \mu \mathrm{m}$ with a $32 \mu \mathrm{m}$ spacing between two channels and a length to diameter ratio of 60 [138]. Their axis are tilted by an angle of $8^{\circ}$ to the normal on the MCP input surface and two multichannel plates are stacked on each other in a so-called Chevron configuration [140]. This enhances the yield and minimizes the chance for secondary ions to drift from the back to the front and disturb the signal. 
Phosphor screen: The electron avalanche exiting the back side of the back MCP is further accelerated towards a phosphor screen and transformed into an optical signal. P20-type phosphor is applied on a fiberoptic plate for obtaining high spatial resolution. It emits green light around $550 \mathrm{~nm}$ wavelength with a decay time in the $\mu$ s range. The visible image on the screen is then recorded by an out-of-vacuum CCD-camera. The linearity and efficiency flatness of this detector are discussed in the appendix to this thesis.

A hole in the center of the detector is necessary to guide the FEL beam out of the interaction region. It leads to an inactive area in the center of the detector with a diameter of about $4 \mathrm{~mm}$. Such an insensitive area is common to all kinds of small angle scattering experiments.

Detector geometry: The arrangement of detectors in the current setup has been displayed in Fig. 3.9. A sketch of the geometry with distances and angles is depicted in Fig. $3.14 \mathrm{c}$. The scattering detector is placed $61.6 \mathrm{~mm}$ behind the interaction region. The electrodes of the time-of flight spectrometer with a diameter of $60 \mathrm{~mm}$ at $12 \mathrm{~mm}$ distance to each other cast a characteristic shadow on the scattering detector. With a diameter of $75 \mathrm{~mm}$ of the sensitive area and an inactive diameter of $4 \mathrm{~mm}$, the scattering detector covers a scattering angle horizontally from about 3 to $31^{\circ}$, and vertically up to $11^{\circ}$. The scattering patterns visible on the phosphor screen are coupled out of the top flange by a $45^{\circ}$ mirror with a center hole which is optimized to the oblong form of the patterns.

Reduction of ion signal and straylight: Besides elastically scattered photons, also high kinetic electrons and ions fly towards the scattering detector. They can add unwanted noise to the scattering signal or even completely bury it. Therefore the front of the MCP is biased with a high negative voltage, which slows down the electrons or pushes them away. In addition, by fast-switching the MCP voltage off early after the pulse, the photon measurement can be finished before the charged particles arrive. This method is referred to as gating. In this experiment, it was even possible to run the MCP in a constant mode and gate most part of the charged particles away by triggering the CCD camera adequately.

Already a single direct hit of the almost focussed FEL beam could seriously damage the scattering detector. While the adjustment operations as described in section 3.2.4 were carried out, the MCP had to be covered with a solid screen plate. Final adjustment steps were taken without the screen using a gradually less attenuated beam. A well adjusted set of straylight apertures defined and guided the beam during operation, also protecting the system in case of slight movements of the FEL beam.

\subsubsection{Ion detection}

In the first paragraph of this section the basic experimental principles of ion time-of-flight spectroscopy are described. The second paragraph will then summarize characteristics of the spectrometer used in this experiment. In the third paragraph, the procedure for optimizing the overlap of the laser and the cluster beam at highest power density using the time of flight spectrometer is discussed.

Time-of-flight spectrometer: For ion detection a time-of-flight (tof) mass spectrometer was implemented in the setup, which is displayed in Fig. 3.9. A system of electrodes accelerates and guides the ions produced in the interaction region towards a time resolving detector. The mass-over-charge ratio of the ions and their initial kinetic energy are determined by their 
flight time.

A tof spectrometer with a single acceleration stage is used in our setup, as depicted in Fig. 3.15 a. The created ions are accelerated over a distance $d_{1}$ by a voltage $U_{\text {acc }}$ applied between two plates. The ions reach a velocity $v_{1}$ which depends in first approximation only on their mass and charge. Through an aperture or a mesh, they enter the field-free drift region of length $d_{2}$, where their different velocities lead to an increasing difference in time of flight and thus to a better resolution. By solving the equation of motion in the acceleration region for $t_{1}$, the moment when the ions enter the drift tube, one obtains

$$
t_{1}=\sqrt{\frac{4 d_{1}^{2}}{U_{a c c}} \cdot \frac{m}{q}} .
$$

$v_{1}$ can be calculated to

$$
v_{1}=a \cdot t_{1}=\sqrt{U_{a c c} \cdot \frac{q}{m}} .
$$

The time $t_{2}$ the ions need to pass the drift region with constant velocity is

$$
t_{2}=\frac{d_{2}}{v_{1}}=\sqrt{\frac{d_{2}^{2}}{U_{a c c}} \cdot \frac{m}{q}} .
$$

As a result, the overall time of flight can be calculated to

$$
\text { tof }=t_{1}+t_{2}=\sqrt{\frac{m}{q}} \cdot \frac{2 d_{1}+d_{2}}{\sqrt{U_{a c c}}}=\text { const } \cdot \sqrt{\frac{m}{q}} .
$$

Bipolar TOF: Fig. 3.15 a displays a scheme of the "bipolar tof" spectrometer, which has been used in this setup. A sketch of the detector mounted on a CF63 flange is displayed in Fig. 3.15b and an image of its components is shown in Fig. 3.15c.

Ions created in the interaction region are accelerated by a voltage of $500 \mathrm{~V}$ over a distance $d_{1}=6 \mathrm{~mm}$ before they enter the grounded drift tube through an aperture with $1.7 \mathrm{~mm}$ in diameter. The aperture opening is comparable to the Rayleigh length of the FEL focus in the order of $1 \mathrm{~mm}$, restricting the interaction volume to the region of highest power density.

After a drift distance $d_{2}=142 \mathrm{~mm}$ the ions pass a mesh and are then post-accelerated over a very small distance towards an MCP to enhance detection efficiency. The energy of the ion impact on the MCP plate is transformed into an avalanche of electrons, as explained in the last section, cf. Fig. 3.14b. For ion measurements the MCP detection probability and the signal intensity in general depend upon the atomic mass, the charge and impact kinetic energy of the ions [141]. For xenon ions with kinetic energies of at least $3.1 \mathrm{keV}$ (cf. voltages in Fig. 3.15a), the detection probability is one, as every ion reaching the MCP ejects several electrons [141]. However, the output signal of the MCP will depend on the number of electrons ejected until at a certain point saturation is reached. Fig. 3.16 displays efficiency curves as a function of ion mass for different impact energies, calculated and measured for carbon based molecules. The curves for xenon might differ, but a first-order estimation can be done based on this graph. The ion with lowest efficiency in the current experiment, $\mathrm{Xe}^{1+}$ is indicated in blue. For an acceleration of $(0.5+2.6) \mathrm{keV}$ the MCP is already operating close to saturation and the signal from ions with finite initial kinetic energy and higher charge states will be amplified even more. Therefore, to simplify the discussion in section 4.4 the MCP detection efficiency will be assumed to be in saturation. 


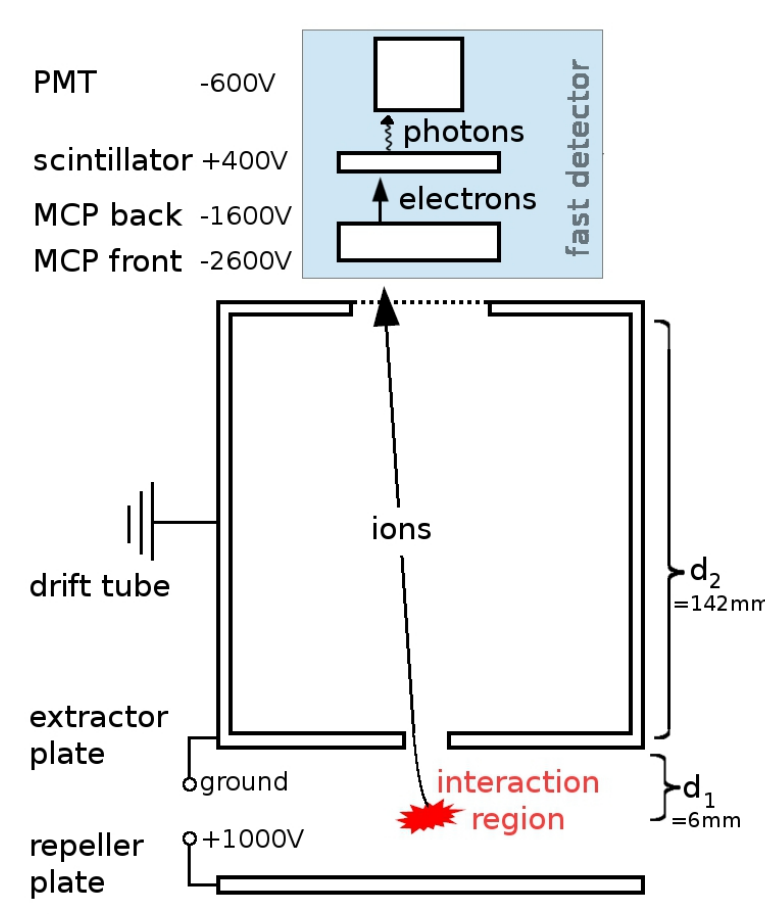

a)

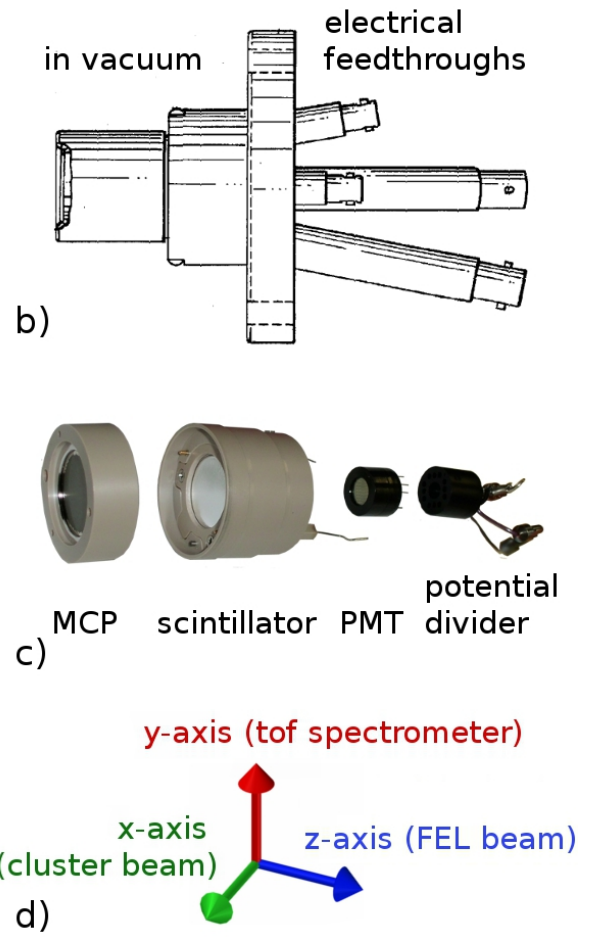

d)

Figure 3.15: Bipolar tof: a) Scheme of ion tof spectrometer with bipolar detector and operation voltages. b) Draft of the bipolar detector mounted on a CF63 flange. c) Components of the bipolar detector assembly. d) Coordinate system of the experiment (see text).

The electron pulse is subsequently accelerated towards the scintillator. A fast photodiode detects the sparks on the scintillator. This signal is coupled out by a potential divider, digitized and stored on a PC. Therefore the digital transient recorder Acquiris [142] is used, providing high-speed signal measurement with a temporal resolution of up to $250 \mathrm{ps}$ and 8 gigasamples per second.

Possible limitations of the mass resolution of a time of flight spectrometer consist in the resolution of the fast ion detector itself, the size of the interaction region and the initial kinetic energies of the ions. By choosing suitable electrode geometries and voltages or by using more than one acceleration stage, the resolution can be optimized to time-focus ions with the same $\frac{m}{q}$ but different points of birth or initial kinetic energy, respectively. This is for example realized for experiments with an extended interaction region in the so called Wiley-McLaren configuration [143].

In contrast, the specific spectrometer used in this experiment was designed in a non-WileyMcLaren configuration. Thereby, initial kinetic energy distributions of the cluster ions can be resolved (cf. section 4.4).

At the smallest focal waist already a very sharp spatial distribution is obtained. However, the broadening of the ion signal for positions far away from the smallest focus diameter can be observed in Fig. 3.17b) in the smeared-out structure of the $\mathrm{Xe}^{2+}$ signal far away from from the focal waist (green spectrum).

From Fig. 3.17, a mass resolution $\frac{\Delta m}{m}$ of 285 can be calculated from the width of ${ }^{132} \mathrm{Xe}^{2+}$ of $0.23 \mathrm{u}$. Thus, with the bipolar tof, in the focus of the FEL beam the isotope structure of xenon can be fully resolved. 


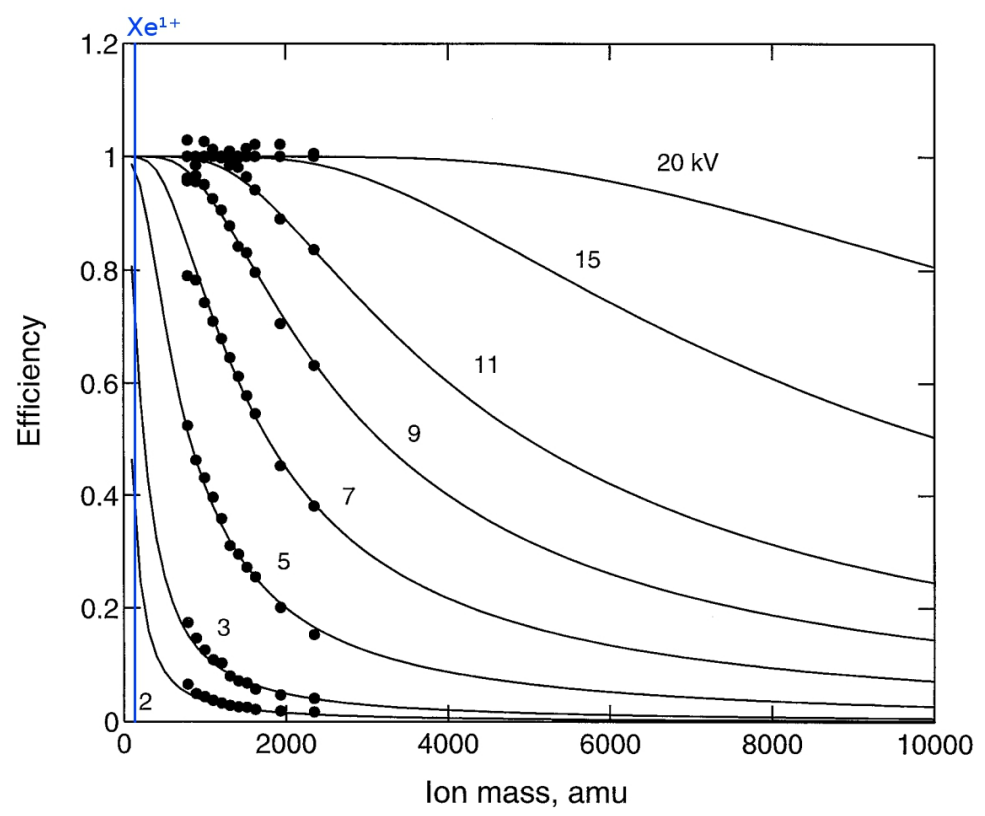

Figure 3.16: MCP efficiency curves as a function of ion mass for different impact energies on the MCP [141]. The lines are calculated efficiency curves, the points are measurements for carbon clusters. In principle the curves might differ for different atomic species. For a simplified estimation, singly charged xenon is indicated in blue. Without initial kinetic energy, the $\mathrm{Xe}^{1+}$ will impact with $3 \mathrm{kV}$ energy, i.e. at $70 \%$ efficiency. The signal from higher kinetic energies and charge states will be amplified more.

Focus optimization and characterization: The interaction region is defined by the overlap between cluster beam and FEL focus. This requires a couple of optimization procedures for which the ion spectrometer is used. At the end of the optimization process, a characterization of the focal power density is carried out using the tof spectrometer.

In this paragraph, optimization and characterization procedures are explained by using a left-handed coordinate system as displayed in Fig. 3.15d: $\vec{x}$ is set on the axis of cluster beam, $\vec{y}$ lies on the axis of tof-spectrometer, and $\vec{z}$ is equivalent to the FEL axis. The interaction region has to be adjusted to the position of the tof aperture and the cluster beam alignment. The alignment procedure for the FEL beam is therefore carried out in three steps:

1. Optimize the position of the FEL beam relative to the tof aperture in $\vec{x}$ direction. Ions from background gas, such as water become apparent in the ion spectrum when the FEL beam is located below the tof aperture.

2. Adjust the hight of the FEL beam to overlap with the cluster beam. The optimal position is found by optimizing on cluster specific signal as singly charged xenon ions.

3. Optimize the focal power density by shifting the FEL focus in respect to the spectrometer along the $\vec{z}$ axis. Optimization is done by scanning for nonlinear charge states, for example of xenon gas, i.e. charge states $>X e^{6+}$ which can not be reached with single photon processes [51].

All three steps require a relative movement of the beam to the interaction region. This experiment was carried out in the beamline focus of BL2 at FLASH, which can not be easily 

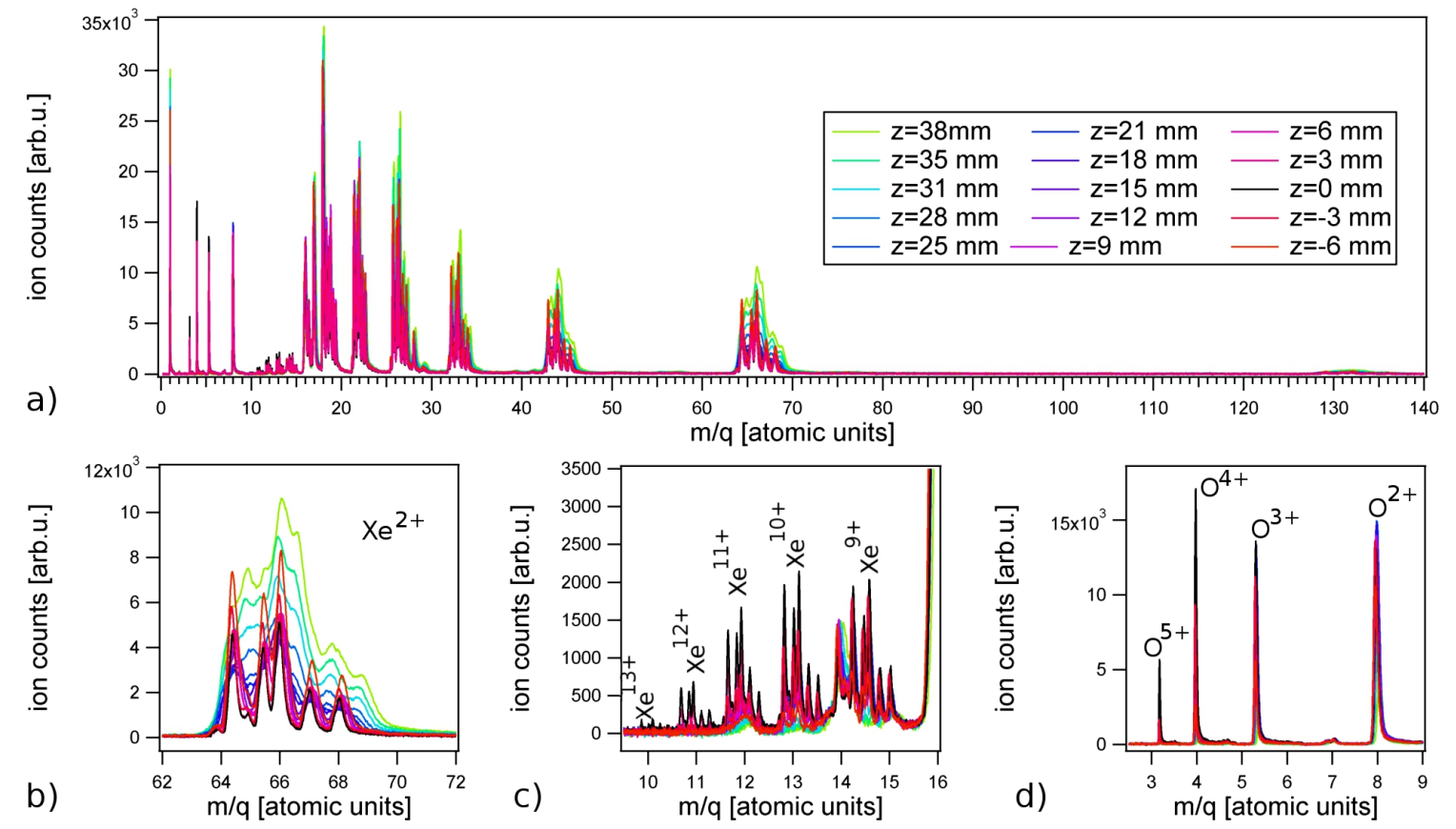

Figure 3.17: a) Time-of-flight spectra of xenon and residual gas atoms for different z-positions of the chamber along the FEL axis. b) Xenon 2+. For positions far away from the smallest focal waist, the increased focal volume results in a poor resolution. c) Position of highest power densities is defined as $z=0 \mathrm{~mm}$. Here, xenon charge states up to $13+$ are observed. d) Higher charge states of oxygen from the residual gas up to $\mathrm{O}^{5+}$ also indicate highest power density for the black curve.

moved. It is more practicable to move the experimental chamber around the FEL beam. Therefore the chamber is equipped with motorized bearings. However, in experiments using a back-focussing multilayer mirror as $[24,125]$, the mirror was tilted or moved along the beam axis.

The results of the focus scan for optimizing the setup in advance to the measurements for this work are displayed in Fig. 3.17. The spectra of atomic xenon for different $z$-positions reveal the position of the black curve to be at highest focal power density. Higher charge states of oxygen from the residual gas up to $\mathrm{O}^{5+}$ also indicate highest power density at this position, which is therefore defined as $z=0$. A maximum charge state of $13+$ for xenon was detected which indicates a focal power density of $\sim 5 \cdot 10^{14} \frac{\mathrm{W}}{\mathrm{cm}^{2}}$ [51]. With a pulse energy of $150 \mu \mathrm{J}$, a focus diameter of $20 \mu \mathrm{m}$ can be calculated. However, it is important to note, that the actual pulse duration in the experiment of $100 \mathrm{fs}$ was about a factor of 5 longer than in the cited reference. This can lead to equally high charge states at lower power densities as the photon flux is higher. 


\section{Chapter 4}

\section{Results and Discussion: Imaging and ion spectroscopy of single large xenon clusters}

\subsection{Introduction}

The results of this thesis are quite diverse and related to different aspects of cluster properties, their interaction with high intensity XUV pulses, and the dynamics of this interaction. In order to investigate these aspects, a combined scattering and spectroscopy approach was used. The two methods, imaging and ion spectroscopy, are sensitive to different parameters and address different timescales, as sketched in Fig. 4.1. Elastic light scattering (Fig. 4.1a) is an instantaneous process. The photons are scattered by electrons, either bound to atoms or quasi-free in the nanoplasma. Thus, information about both, the initial state of the clusters and changes in the clusters during the interaction on a femtosecond timescale are encoded in the scattering patterns. On the other hand, ion spectroscopy (Fig. 4.1b) can yield complementary information on the interaction process. The ion spectra are a result of ionization
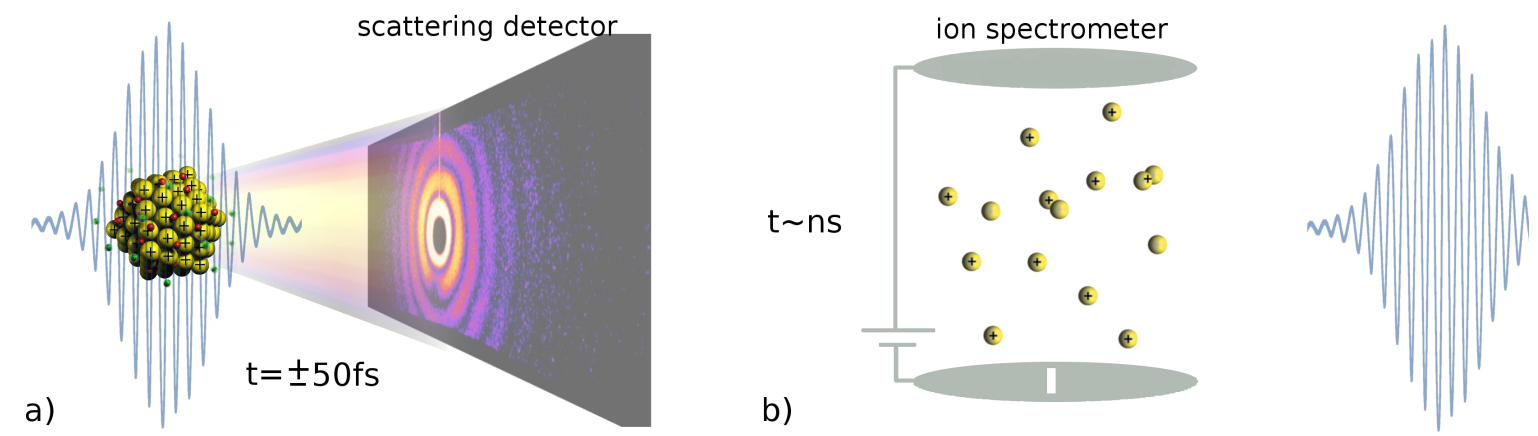

Figure 4.1: Simultaneous imaging and ion mass spectrometry gives access to different timescales within one experiment. a) From the scattering pattern of a single cluster, its size and shape before the interaction can be deduced. Also electronic changes of the particle during the interaction with the pulse, i.e. on a femtosecond timescale, are encoded in the scattered light. b) The ion spectra of a single cluster yield insight in the interplay of ionization, recombination and expansion, taking place on a timescale up to nanoseconds. 

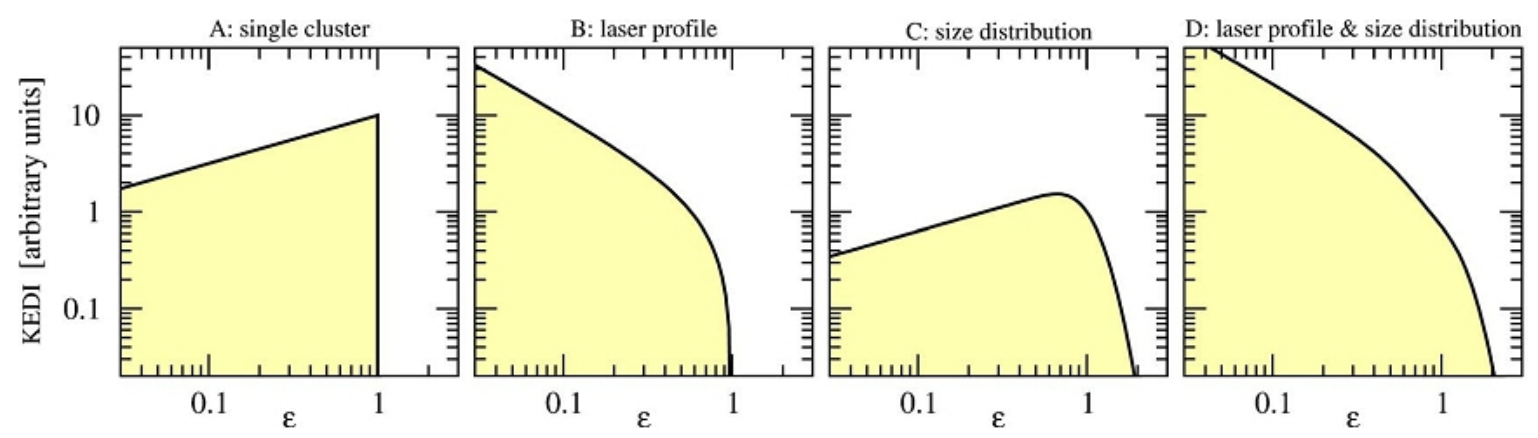

Figure 4.2: The kinetic energy distributions of the ions (KEDI) in Coulomb exploding clusters are calculated (A) and the effects of laser profile (B) and size distribution (C) included (D accounts for both). Even though some features remain, the characteristic structure smears out. From [96]

and recombination. The kinetic energy distributions give insight into charge distributions and expansion processes, taking place on a timescale of nanoseconds.

The high intensities provided by the FEL pulses makes it possible to carry out such measurements on a single cluster in a single pulse. This offers great advantages compared to virtually all previous studies on rare gas clusters, where ensembles of clusters have been probed. An overview of such experiments is given in section 2.3.3. An ensemble consists of a large number of clusters with different sizes which are irradiated by the whole focal intensity profile. Therefore size and power density dependent effects are considerably blurred by the distributions involved. The results from a theoretical study [96] exemplifying the effects of size and power density distributions on the spectra are displayed in Fig. 4.2. The kinetic energy distributions of ions (KEDI) from Coulomb exploding clusters were calculated by stepwise including the effects of laser profile and size distribution. The characteristic features of a positive slope and a clear cut off energy, visible in Fig. $4.2 \mathrm{~A}$, are almost completely washed out in the resulting distribution in Fig. 4.2 D.

Within this thesis it was possible for the first time to produce and study single xenon clusters with radii of several hundreds of nanometers. Such large clusters are interesting objects for several reasons. First, these systems yield a strong and thus meaningful scattering and ion signal even in single particle mode because of the high number of contributing atoms. Second, for xenon at a photon energy of $90 \mathrm{eV}$, the typical length scales of the interaction, such as the penetration depth of the light, are considerably smaller than the particle diameter. Therefore, they appear as radial inhomogeneities in the ionization dynamics and can be investigated. Third, information on radially changing properties can be retrieved from the scattering patterns, as the clusters are a factor of 10 to 100 bigger than the wavelength and signal up to high q-values is visible in the images.

In this chapter, results on single large xenon clusters are presented in the following order: In section 4.2, important properties and requirements of the statistics of single cluster experiments are discussed and the structure of the data and pre-analysis procedures are described. A systematic characterization of the morphology of large xenon clusters including new findings on the growth process and the time structure of pulsed jets are presented in section 4.3. The interaction of single XUV pulses with the large clusters is analyzed in sections 4.4 and 4.5. The ion spectra (4.4) give evidence for highly charged ions being ejected from the surface of an otherwise quasi-neutral and efficiently recombining cluster plasma. The corresponding size selected scattering patterns (4.5) reveal intensity dependent modulations in the scattering signal. They can be traced back to the presence of a refractive core-shell system during the interaction. 


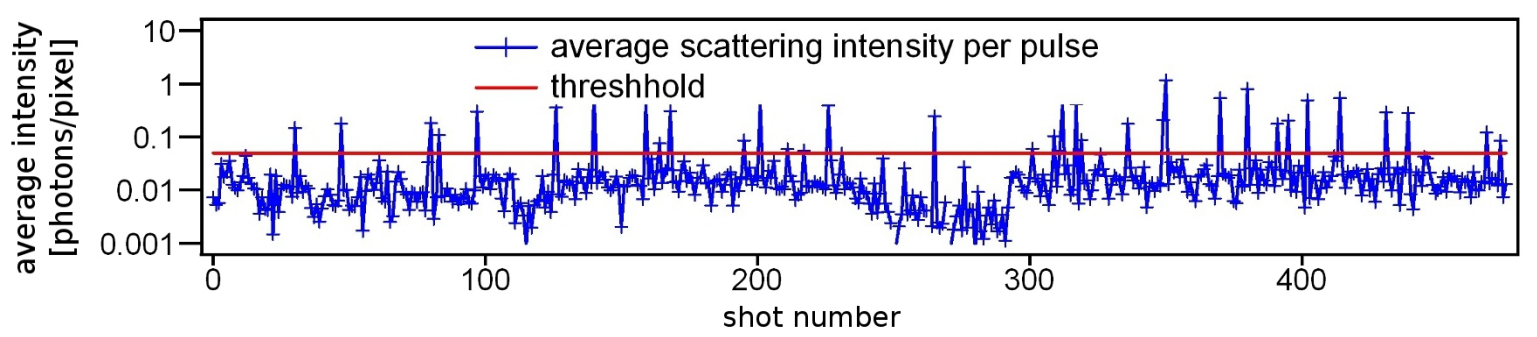

Figure 4.3: The average scattered light per pulse in an exemplary data set of 500 shots indicates single-cluster mode. The red line constitutes the threshold between hits and the background noise.

\subsection{Measurements in single-particle mode}

\subsubsection{Statistics in single-particle mode}

Studying individual particles implies a number of requirements on sample preparation and measurement statistics as well as on data acquisition and analysis. Single-particle mode is experimentally achieved by skimming the cluster beam down, until a large fraction of the shots produce no scattering signal. Then bright scattering patterns will show only one single cluster with a high probability. Such data points with bright patterns are referred to as hits in the following discussion.

The term single-cluster mode has been introduced in [24] for an exemplary data set of 500 scattering patterns of single clusters, as displayed in Fig. 4.3. The clusters were produced at a stagnation pressure of 14 bar xenon gas and a temperature of $220 \mathrm{~K}$. Here, the average number of photons per pixel on the scattering detector is plotted for every pulse of this data set. Only 34 bright patterns out of 500 shots peak out of the background noise $(7 \%$ hits), clearly indicating single-cluster mode. The threshold to discriminate between a hit and background noise (red line in Fig. 4.3) was set to 0.05 scattered photon per pixel, which is high above the noise level.

In every experiment and at every new set of pressure and temperature settings, single-cluster mode settings have to be reestablished, as the number of clusters per volume depends on the expansion parameters. The limit of single cluster mode is somewhat arbitrary, but as a basic rule, up to a ratio of 30 percent bright patterns, a data run can be considered to be in single cluster mode. From the Poisson distribution, the probability for two clusters in the focus can then be calculated to $1 / 6$ of the bright patterns. Newton Rings as another signature for leaving single cluster mode are discussed at the end of this section.

In a single-particle experiment, in principle every hit may be considered a whole data set. Nevertheless, the requirements on data acquisition time for each parameter set rise compared to experiments on ensembles. This is because the comparability of the hits among each other is lost with poor statistics. In other words, the number of hits at one distinct cluster size has to be high enough to map the whole focus profile. Only if sufficient hits at different intensities are available in such a data set, intensity dependent evolutions become visible and unambiguous. The power densities the individual similarly sized clusters were exposed to can be assigned by normalizing the brightest hit with the maximal focal power density and allocating the others relatively to it. By carefully comparing hits from clusters exposed to similar power density, deviations due to artifacts can be identified. 
The demanding requirements to measurement statistics are particularly valid for pump-probe experiments, where fluctuations in intensity and overlap of both pulses have to be considered additionally.

In the beamtime in July 2011, approximately 0.5 million single shots were acquired. All data obtained with a single XUV pulse (pump-probe data using XUV double pulses excluded) contain together only about 1000 scattering patterns of single clusters with an assignable cluster size (cf. section 4.3.1). For larger clusters with a radius $R>60 \mathrm{~nm}$, the best statistics were obtained around $400 \mathrm{~nm}$. This data set of about 100 hits with $R=400 \pm 50 \mathrm{~nm}$ constitutes the basis of the detailed analysis of intensity dependent evolutions in ion spectra and scattering patterns, cf. section 4.4 and section 4.5 , respectively.

\subsubsection{Data acquisition and processing}

The data in this experiment consist of single events with several different coincident measurements belonging to each event. In particular a scattering pattern, the corresponding ion spectrum and further experimental parameters, such as FEL pulse energy or gain settings of the detectors have to be combined. They are stored on a shot-to-shot basis together with a unique pulse identifier, the so called bunch-ID, with the data acquisition program FoxIT [144].

Already one single event sums up to a considerable amount of data. Further, the data structure varies from a 2D matrix with $1394 \times 1040$ pixel from the CCD camera, over $1 \mathrm{D}$ vectors from the Acquiris with 20000 to 40000 elements to single values in case of the meta-data. In order to manage these large volumes of data of different type, a special data format, HDF5, is used [145].

The data files created have the structure of a folder with subfolders, with one file for each experimental run. Constant settings are written into a header folder and the shot folders contain a consecutive Acquiris ID number, four acquiris channels, a 16 bit CCD image, a consecutive CCD ID number and the bunch-ID. For the analysis presented in this thesis, Matlab based analysis algorithms were used to handle and sort the data.

Successful analysis strategies for data from simultaneous imaging and ion spectroscopy experiments on single clusters have proven to be a combination of manually evaluating unprocessed data and applying appropriate filter algorithms. In a pre-analysis step, all data is automatically catalogued for distinct criteria. In particular, those criteria are

- total intensity on the scattering detector

- total intensities of detector sections at small and large scattering angles

- intensity of the scattered light peak in the ion time-of-flight spectra

- intensity of the $\mathrm{Xe}^{+}$peak in the ion time-of-flight spectra

- integrated intensity in the ion time-of-flight spectra from higher Xe charge states

Those parameters are stored for each shot in run matrices. In subsequent analysis steps, filter algorithms scan the run matrices for hits, which can be defined as different combinations of parameters.

For all hits radial profiles are extracted from the scattering patterns. They are smoothed and scanned for local maxima and minima to determine the cluster size. Especially here, iterative manual checks of the results and subsequent adaption of the filter parameters are essential. 
a)
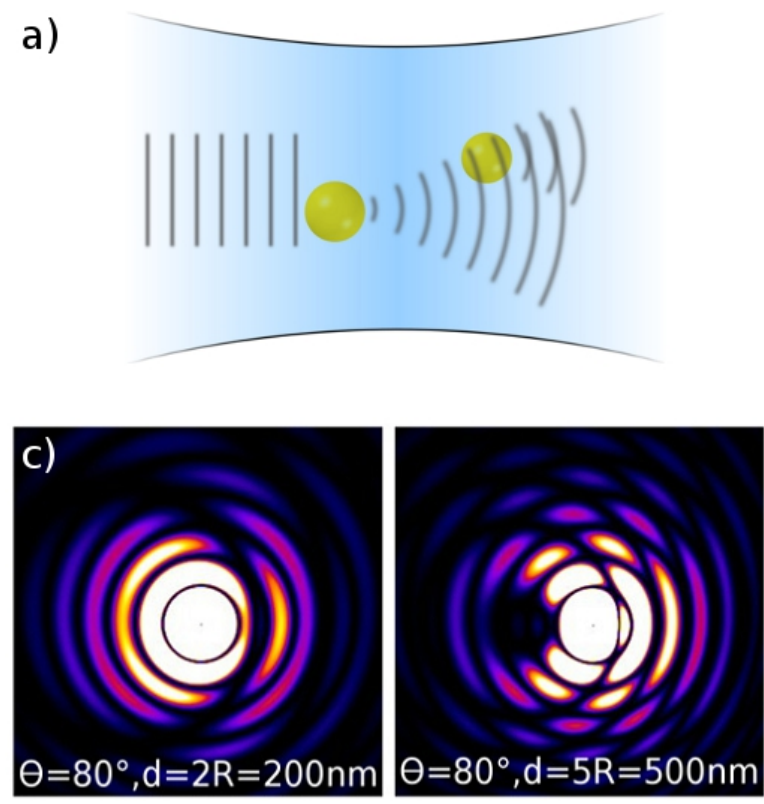
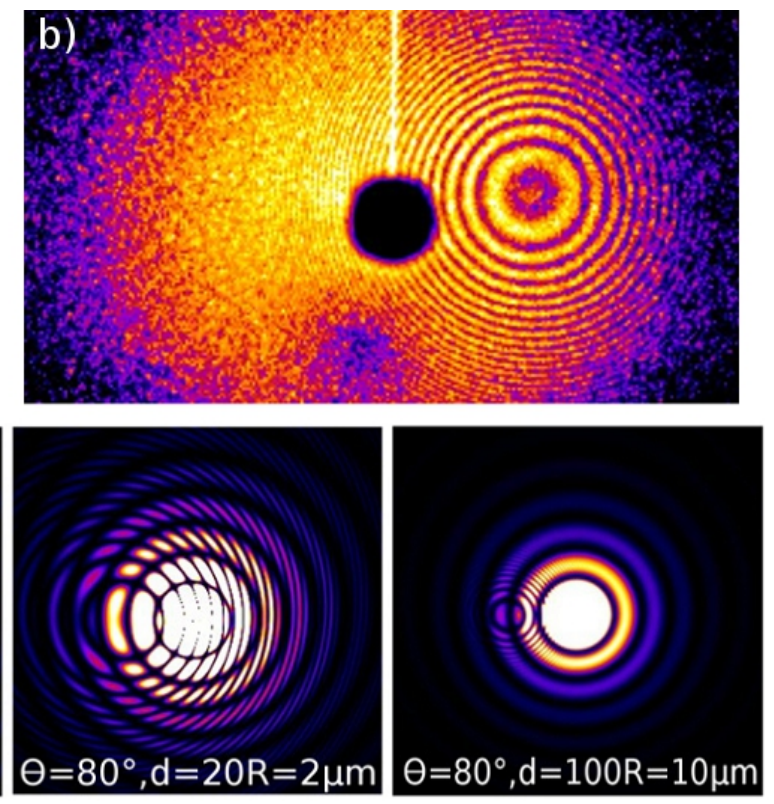

Figure 4.4: Newton rings: a) The scattered light of two clusters, one after the other, in micrometer distance interferes. b) Experimental scattering pattern with Newton rings. c) Simulated scattering patterns for two clusters one after the other with increasing distance, see also [24].

\subsubsection{The limit of single particle mode: Newton rings}

In the transition region between pure single-particle mode and measurements on ensembles of clusters, more and more frequently a fine structure appears in the scattering patterns. Due to the resemblance with the interference effect known from almost parallel refracting surfaces, this structure is called Newton rings. The occurrence probability for Newton rings strongly depends on the cluster density in the focal volume and thus on the chosen expansion parameters and the skimmer slit opening. Therefore their appearance is an indication for leaving single cluster mode.

An example of such a Newton ring pattern is displayed in Fig. 4.4 b. The formation of Newton rings is sketched in Fig. 4.4 a. When two clusters, one after the other, are illuminated at a certain distance, Newton rings superimpose the basic scattering pattern alongside the axis that intersects both clusters.

These Newton rings allow for extracting three dimensional structural information out of a single scattering pattern. The center position of the Newton rings represents the orientation of the cluster axis with respect to the incoming beam, while the separation of both clusters can be determined from the distance of the fringes.

The emergence of the typical interference structure of Newton rings with increasing distance between both clusters can be traced in Fig. $4.4 \mathrm{c}$. Simulated scattering patterns of two clusters at an angle of $10^{\circ}$ between FEL beam and cluster axis $\left(80^{\circ}\right.$ between cluster axis and scattering plane) exhibit finer and finer interference fringes with increasing distance between both particles. For all calculations of three dimensional configurations of two spherical particles in this work (Fig. 4.4c, Fig. 4.5 and Fig. 4.8 a), the simulation program ClusScat8 was used. ClusScat8 is an adapted version of the program ClusScat3 [146]. It is based on a numerical approach with a Guinier-like scalar approximation of the scattering process from 
an arrangement of point scatterers. By matching experimental patterns with simulated ones, the three dimensional configuration of clusters in the focus can be retrieved.

Throughout all data, Newton rings appear only at small scattering angles. This provides a direct evidence of the short coherence length of the light from the SASE driven FLASHFEL. The scattering waves of two clusters can only interfere if the path difference for the scattered light from both clusters remains within the coherence length. The coherence time of FLASH has been determined to be 4.2 fs [108]. Autocorrelation measurements with the split and delay unit available at FLASH $[113,147]$ show it to be less than 6 fs. A value of $4.2 \mathrm{fs}$ would allow a path difference for the scattered light of both clusters of maximal 1.3 $\mu \mathrm{m}$ before interference is lost. Therefore the observation of Newton rings is limited either to small angles between the cluster axis and FEL beam or short distance between both clusters, respectively.

In Fig. 4.5 a match of experimental and simulated pattern is displayed. The experimental scattering pattern exhibits a Newton ring structure with the center on the lower left side center hole of the detector. On the upper right side of the center hole, a second, weaker and slightly distorted structure can be seen. This structure is due to the Moirée-effect from the pixels of the CCD camera. As the simulation program calculates the scattering pattern pixel by pixel, it also reproduces those Moirée patterns. The simulation shown in Fig. 4.5 was calculated for a cluster radius of $330 \mathrm{~nm}$, an angle between cluster axis and FEL axis of $6^{\circ}$, a distance between both clusters of $70 \mu \mathrm{m}$ and a pixel size of $69 \mu \mathrm{m}$. Also because of the short coherence time, Moirée structures as in Fig. 4.5, which should appear in every NewtonRing pattern, remain very rare. Throughout all data of this work, only three patterns were obtained. All three examples revealed the Moirée structures at very small scattering angles, however, symmetry to the center position of the pattern, as observed in Fig. 4.5, is not a necessary condition.
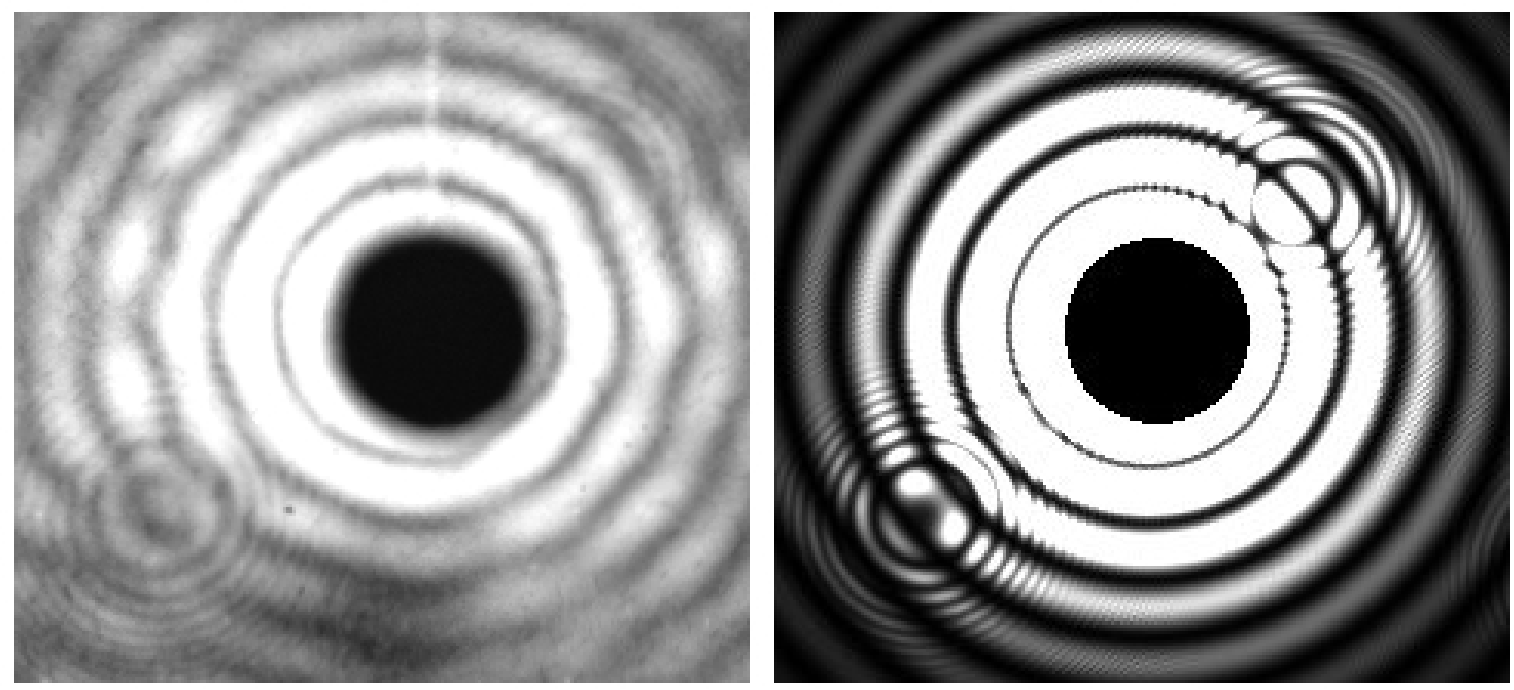

Figure 4.5: Measured and simulated scattering patterns superimposed with Newton rings and additional structures due to the Moirée effect from the pixels of the camera. The simulation parameters for the calculated pattern are $\mathrm{R}=330 \mathrm{~nm}, \Theta=6^{\circ}, \mathrm{d}=70 \mu \mathrm{m}$ and a pixel size of $69 \mu \mathrm{m}$. 


\subsection{Information on cluster morphology in single cluster scat- tering patterns}

In this experiments rare gas clusters are used as ideal model systems for studying lightmatter interaction. They are easy to produce, size scalable, and their simple electronic structure makes them preferable for theoretical approaches: single atoms with either few or many electrons are bound by the non-directional, weak van-der-Waals force. Rare gas clusters are also assumed to have a rather simple geometrical structure of icosahedra in the case of small clusters $(N<800)[148]$ which grow into spheroidal particles with an fcc lattice with increasing size [149].

Imaging single clusters in highly intense short-wavelength pulses has now enabled us to gather more direct information on size distributions, cluster morphology and growth processes. From the scattering patterns we can extract the shape and size of a single cluster. On the one hand, this leads to better characterization of the system and thus enables correct interpretation of the data. On the other hand, deeper insight into the formation of clusters has led to the advancement of experimentally accessible parameters.

The used wavelength at FLASH of $13.5 \mathrm{~nm}$ constitutes the resolution limit for imaging, therefore only large clusters are suitable for this type of experiment. This requirement has pushed the use of more extreme nozzle forms and expansion parameters in our experiment. Meanwhile for the production of rare gas clusters, a size range up to $1 \mu \mathrm{m}$ has become accessible.

The changing shape of clusters from spherical via twin- and triple-structured to hailstone-like particles with increasing diameter is discussed in section 4.3.1. A deeper understanding of the structural development is gained by detailed studies of simulated twin-patterns. The comparison of experimental scattering patterns with matching calculated images demonstrates that the scattering patterns are snapshots of cluster growth.

The formation of such giant clusters appears to be related to the pulse shape of pulsed jets. The observed size distributions in the cluster pulse at different times after the valve opening and possible explanations for the emergence of cluster sizes far above known scaling laws [33] are discussed in section 4.3.2.

\subsubsection{The shape of clusters changing with size}

A wide diversity of structures can be found in the scattering patterns of xenon clusters. In Fig. 4.6 a selection of scattering patterns from the whole size range is presented.

As the scattering process is basically equivalent to the Fourier transform of a real space object, the angular distribution of the scattered electromagnetic wave contains the structure of the imaged particle. But only the averaged intensity distribution of the scattering pattern is measured and the phase information is lost in the detection process. Approaches exist to retrieve the phase from the scattering pattern by iterative algorithms, referred to as reconstruction [150]. The disadvantage of this method is that the computational procedure is complex and by no means successful for all kinds of scattering patterns. In the case of the patterns displayed in Fig. 4.6 the greatest difficulty consists in the large insensitive area at small scattering angles and the restricted measured solid angle.

In the framework of this thesis another approach was taken: By systematically sorting and classifying the images and carefully comparing them with simulated scattering patterns, suc- 

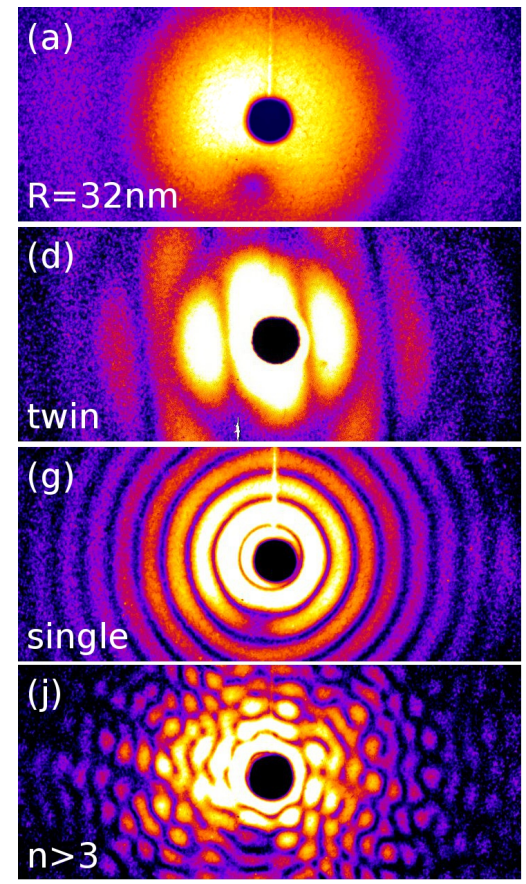
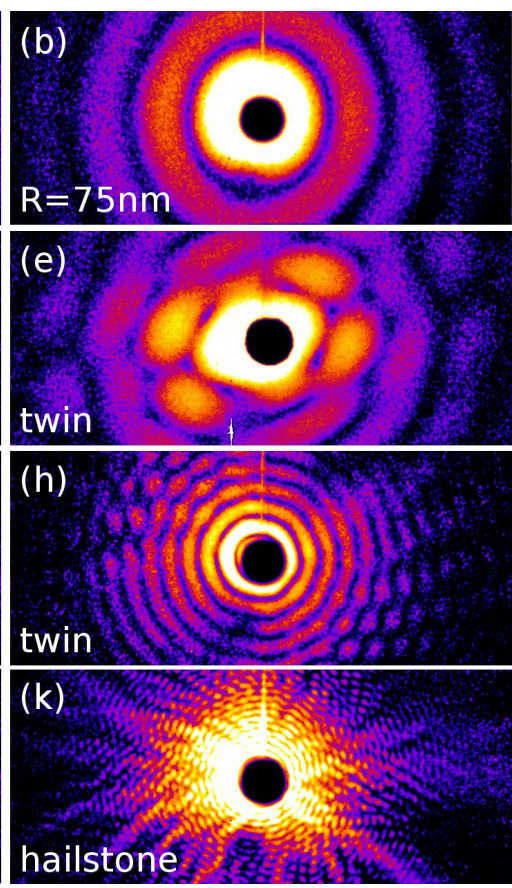
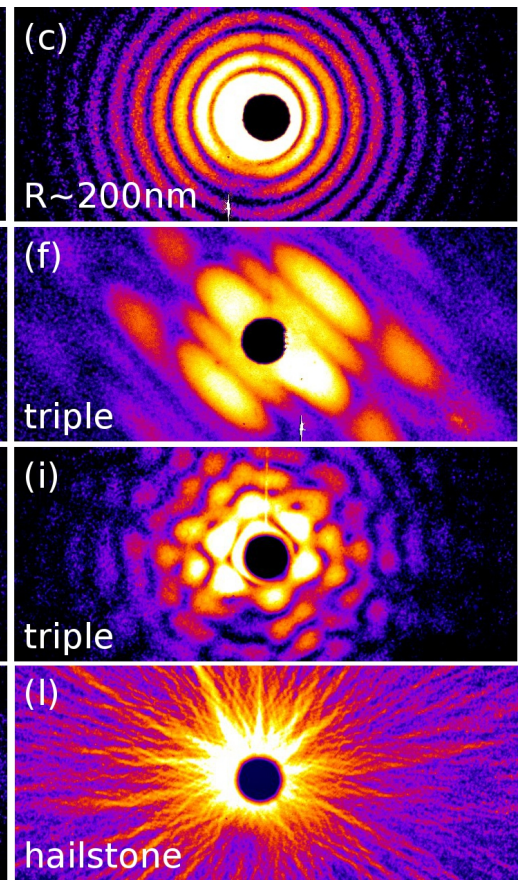

Figure 4.6: Selection of scattering patterns of all shapes from single clusters in the size range of $R=30$ to $700 \mathrm{~nm}$. a-c) Smooth ring structures appear up to $R=200 \mathrm{~nm}$, indicating approximately spherical shape. d,e) 10 to $30 \%$ of all single cluster patterns in the size range up to $100 \mathrm{~nm}$ exhibit twin structure [24]. f) Very rare pattern of a triple cluster of about $50 \mathrm{~nm}$ radius. g-j) For larger clusters $(R=150-300 \mathrm{~nm})$ a transition from spherical via twin and triple structures towards more complicated particles is observed. k,l) Large clusters from $300 \mathrm{~nm}$ up to and exceeding $R=700 \mathrm{~nm}$ radius exhibit complicated patterns indicating a hailstone-like grainy substructure.

cessively a more profound understanding of the cluster structure and its development with size could be gained [24].

The most simple and thus easiest analyzable structures are concentric rings resulting from spherical clusters. Examples thereof are displayed in Fig. $4.6 \mathrm{a}, \mathrm{b}, \mathrm{c}$, and g. Analog to the diffraction image of a pin hole, the cluster size can be retrieved from the position of the maxima and minima. Up to $100 \mathrm{~nm}$ radius concentric rings are the structure with highest abundance. At $R \approx 50 \mathrm{~nm}$ about $\frac{3}{4}$ of all clusters are almost spherical.

It is important to note, that the term spherical is not unambiguous here and directly connected to the cluster size dependent resolution of the scattering images, as illustrated in Fig. 4.7. The structural information is encoded in the pattern inverse to the scattering angle, or more precisely to the momentum transfer $q$ of the elastic scattering process. In the central part of the image, the overall shape and size are contained. Substructures in the particle such as surface roughness correspond to the structure of higher maxima of the scattering pattern. The concrete example in Fig. 4.7 shows a round particle with a finite surface roughness by means of a round mask with 100 pixels in diameter. In this case the surface roughness is defined by the pixel size. The innermost 9 rings of the $2 \mathrm{D}$ fourier transformation, as displayed in the middle panel appear to be perfectly round. In contrast, the outer part of the pattern in the right panel exhibits speckles which give evidence of the surface structure.

Therefore, in the case of clusters of up to $100 \mathrm{~nm}$ in radius, only strong deviations from the spherical form can be observed in the current experiment, where only small scattering angles have been measured. The scattering patterns which deviate from a purely round shape, can be identified as twin clusters, i.e. two spherical clusters sticking to each other. Coagulation, 

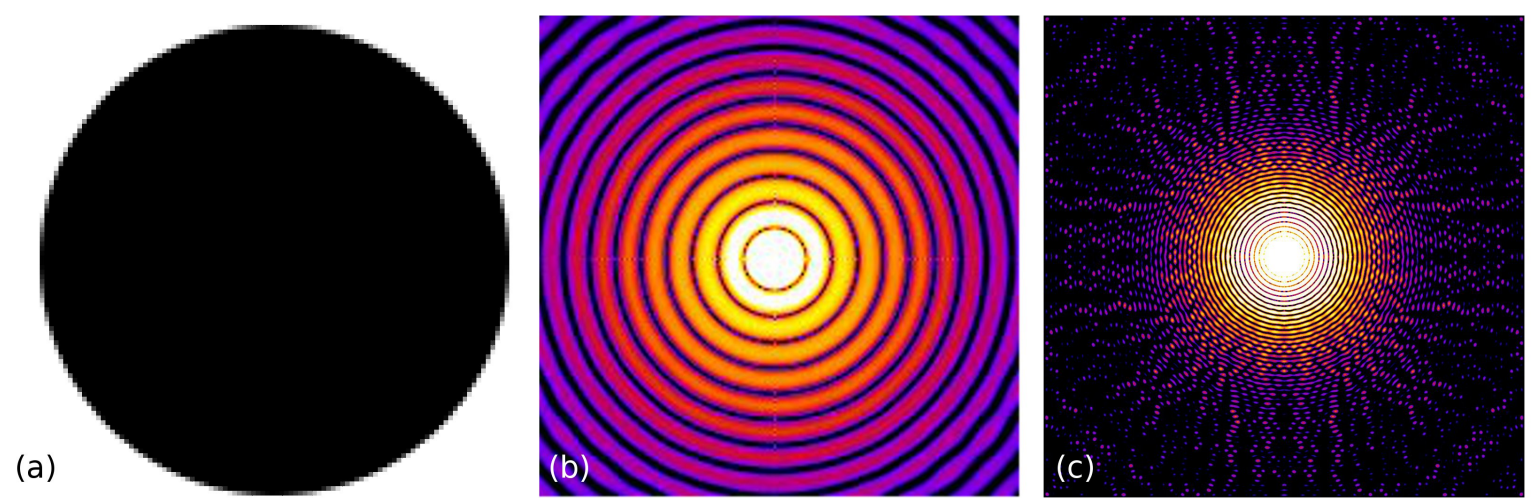

Figure 4.7: The meaning of "spherical". (a) A round mask with a diameter of 100 pixels, i.e. a surface roughness of $1 \%$ of the diameter, is the basis of a simulated scattering pattern. (b) At low q-values the particle appears to be perfectly spherical, the innermost nine rings are smoothly round. (c) At high q values, speckles are the evidence of the surface structure.

i.e. collision of smaller clusters which merge into a bigger cluster, is known to be the leading growth process for large clusters (cf. section 3.2.2). Before imaging of single clusters became accessible it was not known whether the newly formed, bigger cluster could relax into the ground-state geometry of a sphere or if an intermediate dumbbell-shaped state would freeze out. The observed twin patterns range from the well known double slit image displayed in Fig. 4.6 d, over all kinds of variations like Fig. 4.6 e up to only slightly distorted spheres as Fig. 4.6 g. An extremely rare structure in the size range of $50 \mathrm{~nm}$ radius are triple clusters, i.e. three particles sticking to each other, the only clearly identified triple cluster in the data of this thesis is shown in Fig. $4.6 \mathrm{f}$.

The assignment of structures is supported by systematic simulations of twin patterns [24]. The simulated patterns are calculated from systematic variations of three- and two-dimensional structures. By careful comparison with the measured scattering patterns, the cluster configurations can be determined. Virtually all detected patterns can be explained by simulations that allow for three degrees of freedom:

1. orientation of the two cluster centers with respect to the scattering plane (Fig. $4.8 \mathrm{a}$ )

2. distance between two cluster centers, i.e. degree of fusion (Fig. $4.8 \mathrm{~b}$ )

3. size ratio of both particles (Fig. $4.8 \mathrm{c}$ )

Starting point for all subsequent discussions is the simple case of the symmetric double slit (or more precisely double-pinhole), which can be found several times in the data. One example of the characteristic structure is given in Fig. $4.6 \mathrm{~d}$, which stems from two similarly sized clusters in direct contact, oriented perpendicular to the FEL axis.

The influence on the scattering patterns of changing sizes and merging ratios of two clusters can be gained in rather basic simulations by Fourier transforming $2 \mathrm{D}$ projections of the twin clusters (Fig. $4.8 \mathrm{~b}$ and c). However, the effect of different angles $\Theta$ between the axis of the twin and the scattering plane has to be calculated with a 3D simulation. Here the same program as for the Newton rings is used, a Guinier-based scalar approximation of the scattering process (cf. section 4.2.3, [146]). Fig. 4.8 a displays the changes in the scattering pattern of two equally sized clusters in direct contact at a rotation out of the scattering plane 
a)
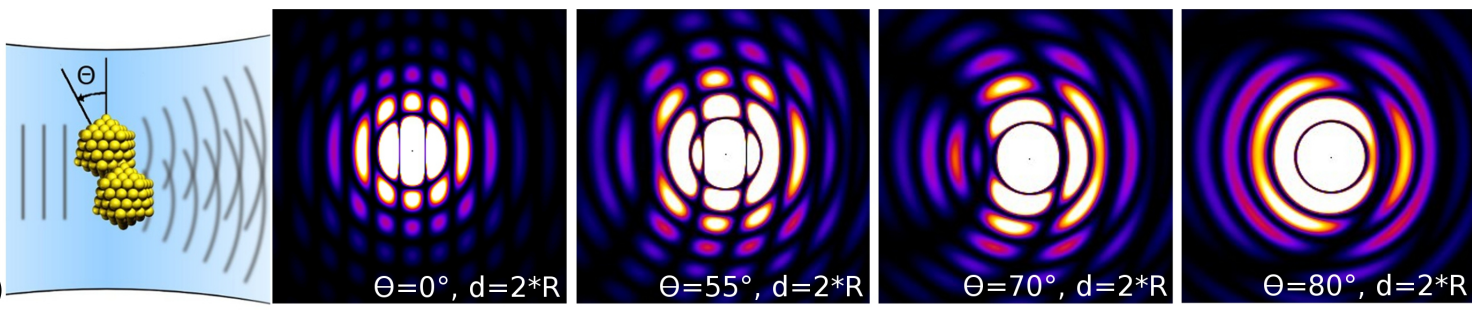

b)
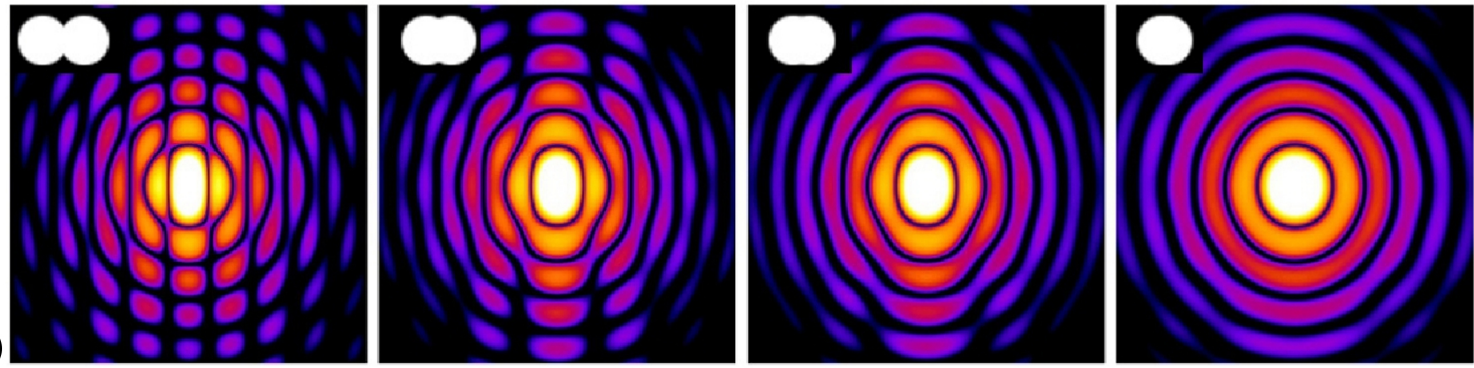

C)
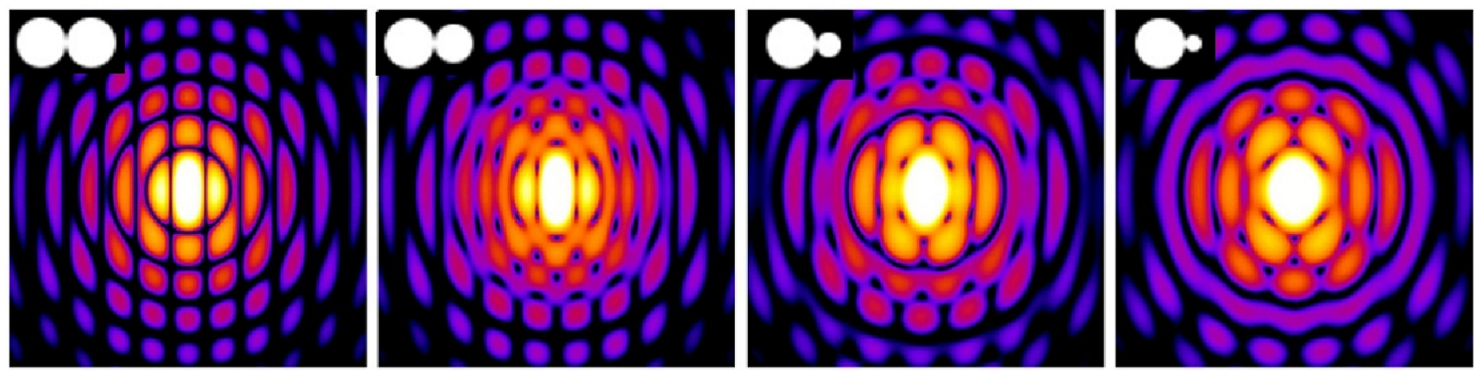

Figure 4.8: a) Simulated scattering patterns of twin clusters for different orientations in respect to the scattering plane. Two spheres of equal size in direct contact are simulated for $0^{\circ}$ (double-pinhole case), $55^{\circ}, 70^{\circ}$ and $80^{\circ}$ b) Different degrees of merging are modeled by Fourier transforming 2D outlines of two equally sized spheres with shrinking distance between their centers. The $2 \mathrm{D}$ outlines are displayed in the upper left corner of each pattern. c) Different size ratios of the two clusters are again modeled by Fourier transforming $2 \mathrm{D}$ outlines of two spheres with different size in direct contact.

of $\Theta=0^{\circ}, 55^{\circ}, 70^{\circ}$ and $80^{\circ}$. The straight fringes of the double-slit-like pattern at $0^{\circ}$ are bent until the dumbbell axis intersects the detector plane and reveals their nature as destructive interference rings around the cluster axis.

Scattering patterns of twin clusters which differ from the case of the simple double slit can be simulated by allowing different radii $R_{1} \neq R_{2}$ of the two particles and different degree of fusion, i.e. shrinking distance between the centers of the two particles $d<R_{1}+R_{2}$. Simulations of examples for both cases are displayed in Fig. $4.8 \mathrm{~b}$ and c. In the upper left corner of the patterns, the $2 \mathrm{D}$ projections are shown on which the calculations were based. In the limits of b) $R_{2} \rightarrow 0$ and c) $d \rightarrow 0$ both cases would end up in the well known ring pattern of a spherical single cluster.

While approaching this limit, in both situations the clearly defined maxima and minima of the sidelobes tend to smear out. In the case of fusion this develops from center to outer rings as can be followed in Fig. $4.8 \mathrm{~b}$ where only the outermost rings reveal facets.

In contrast, for the case of different sizes the substructure differs from ring to ring, depending on the size ratio of the two spheres and the subsequent interference. At a size ratio of $R_{1} / R_{2}=3$, displayed in the last simulation of Fig. $4.8 \mathrm{c}$, the third ring is rather homogeneous while the second and third maximum show pronounced substructure. 

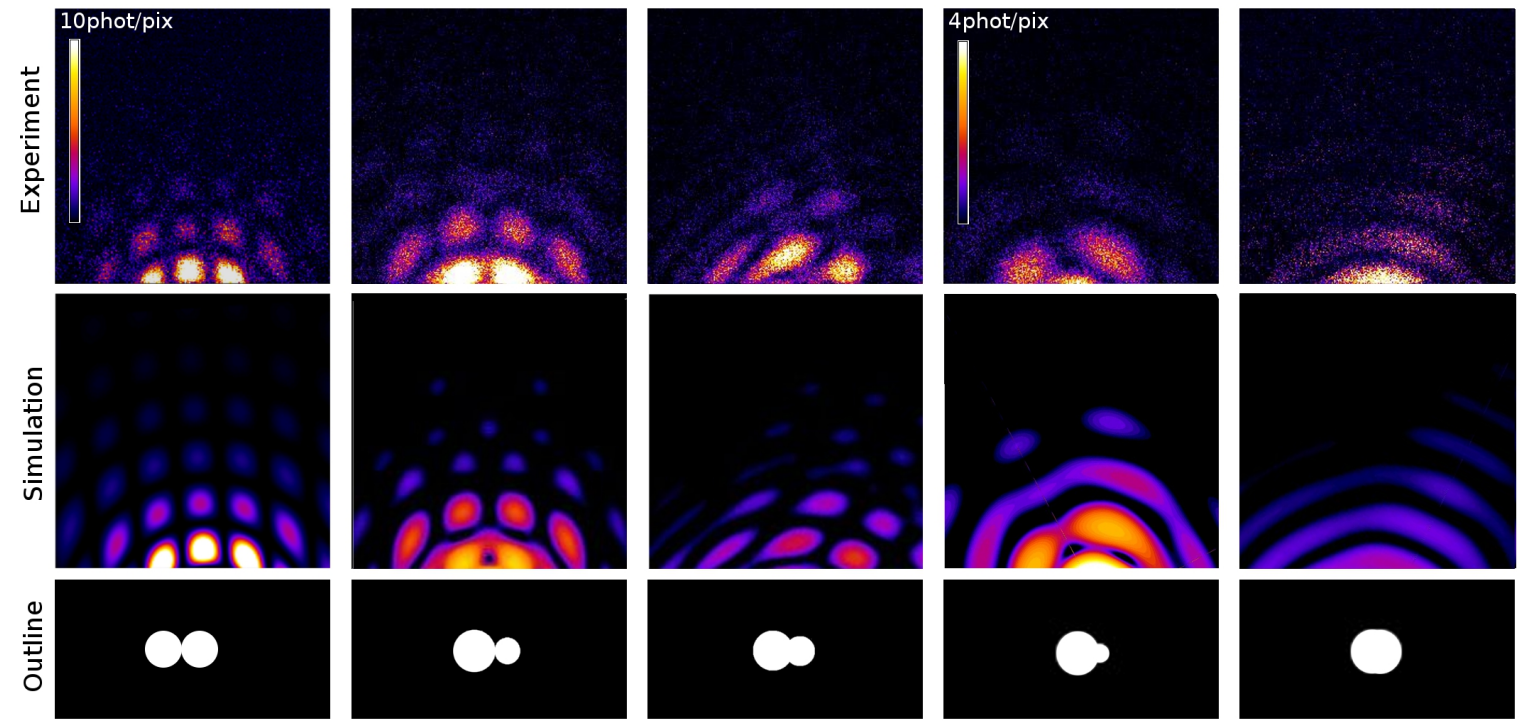

Figure 4.9: Comparison of experimental and simulated patterns of twin clusters. By allowing for different sizes and degree of merging, the experimentally obtained twin patterns in the first row can be matched with simulated ones, as displayed in the second row. The outlines used for the simulations are presented in the third row. Different degrees of merging from direct contact to almost spherical indicate that the scattering patterns are snapshots of the cluster growth by coagulation, where nonspherical shapes can freeze out.

Based on these studies, an attempt can be made to find matches for experimental scattering patterns and thus to identify the underlying particle structure. A selection of experimental twin patterns is displayed in the first row of Fig. 4.9. Those patterns have been measured in a previous experiment [24], where smaller scattering detectors were used [151].

A comparison with the simulated patterns shows that a very good agreement can be obtained and that the fine structure is reproduced when size and degree of fusion are taken into account (middle row of Fig. 4.9). The black and white masks with the outline of the particles used for the Fourier transformations are displayed in the third row of Fig. 4.9. From the finding of various degrees of fusion from two clusters in direct contact to one slightly nonspherical structure it can be concluded, that interstages of the cluster growing process driven by coagulation of particles can freeze out and can be detected milliseconds after the expansion process.

This trend continues towards larger cluster sizes. More and more clusters aggregate and form bigger clusters without relaxing into spherical ground state. Up to a radius of $200 \mathrm{~nm}$, spherical clusters do exist, however, the pattern shown in Fig. $4.6 \mathrm{c}$ is the largest spherical cluster found in all data. In the size range of 100 to $300 \mathrm{~nm}$, some patterns exhibit a symmetry, which indicates a twin (Fig. $4.6 \mathrm{~h}$ ) or triple (Fig. $4.6 \mathrm{i}$ ) structure. However, most patterns have a more complicated substructure in the interference maxima, which can not be identified by simple symmetries. In their grainy substructure, those clusters resemble hailstones and are therefore referred to as such.

Systematical simulations of all possible degrees of freedom already meet their limit at a particle number of $n=3$, in particular because virtually all systems have $3 \mathrm{D}$ structures which can be no longer approximated by 2D projections.

Therefore, simulated patterns for the more complicated structures f, k and l of Fig. 4.6 from 2D masks as displayed in Fig. 4.10 hardly yield good matches. While the simulation of the twin and triple clusters are in reasonably good agreement with the experimental pattern, the 

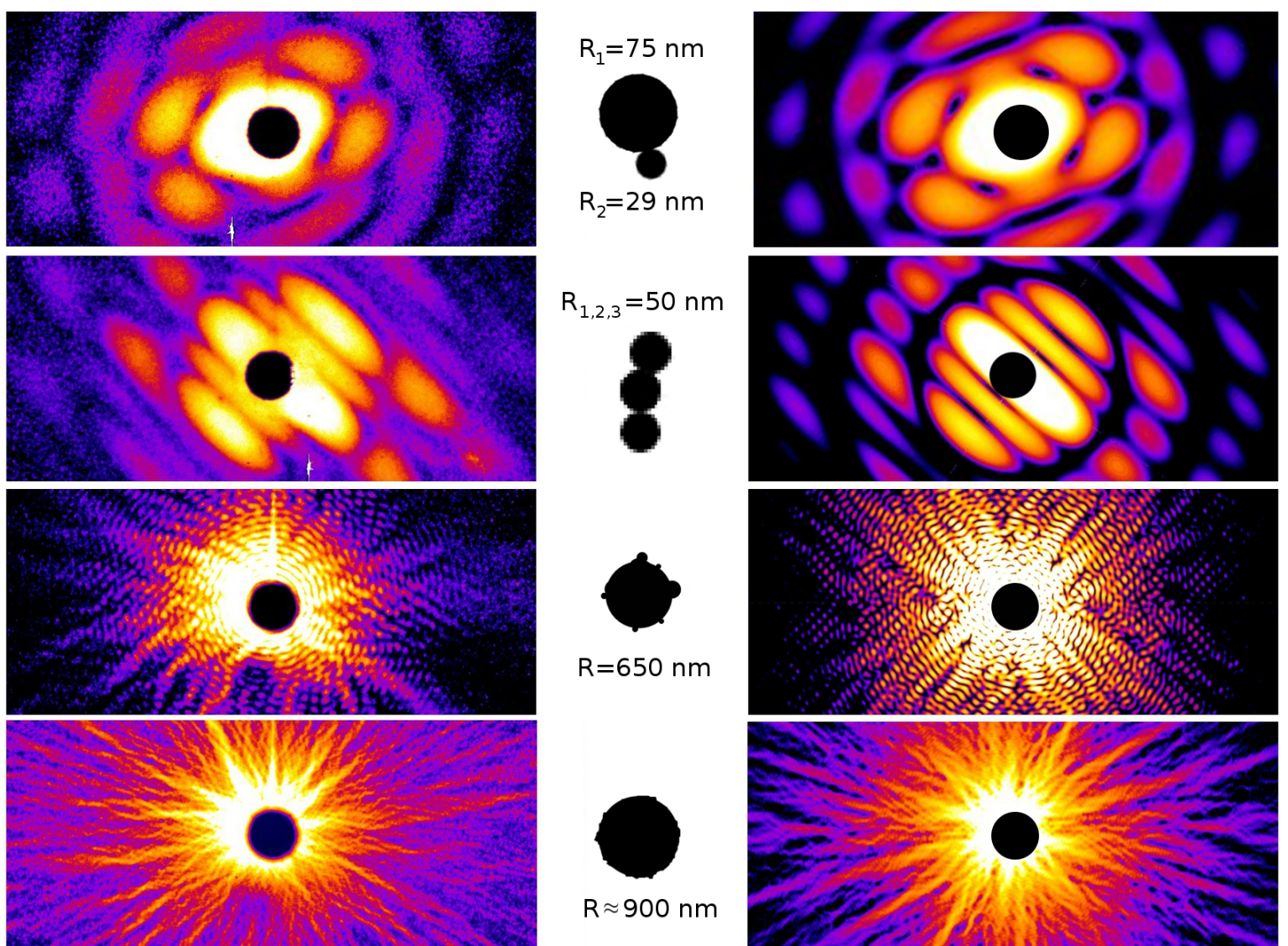

Figure 4.10: Comparison of experimental and simulated patterns of twin, triple and hailstone structures. The middle row displays the outlines, which were used for the simulation. See text for details.

simulations for the hailstones yield only the main features but thereby can give an idea of the particles' structure. The hailstone in the third row of Fig. 4.10 has a radius of approximately $600 \mathrm{~nm}$. While cluster sizes up to $700 \mathrm{~nm}$ radius can be still determined, for clusters exceeding this size the ring spacings get too fine and can not be resolved any more. Therefore the giant cluster in the last row of Fig. 4.10 can only be estimated to have a radius in the $\mu \mathrm{m}$ range. It is interesting to note, that maximal deviations of the overall shape from the spherical case appears around $50 \mathrm{~nm}$ radius with their twin and triple structure. In contrast, the largest hailstones (last row of Fig. 4.10) can be nicely fitted by almost spherical outlines. The grainy substructure can only be seen as a slightly structured surface of an otherwise round particle. Therefore, the simulated patterns in Fig. 4.10 might give a hint towards a characteristic size of the grains of $\approx 50 \mathrm{~nm}$, but further simulations are necessary to confirm this hypothesis.

In conclusion, the experiments have revealed a characteristic formation process for rare gas clusters in this so far unexplored size range. They aggregate from smaller particles but keep the grainy structure instead of returning into the global minimum of a spherical compound. This observation draws a parallel to the characteristic behavior of small rare gas clusters in the transition region from icosahedral to fcc lattice structure. The transformation of rare gas clusters from a pentagonal nanocrystal into the extendable fcc structure on a microscopic level was investigated by van de Waal [149], who proposed, that small particles with a fivefold symmetry which aggregate will favor fcc lattice growth in the crossing region of the twinned crystal seed. He concluded, that "the observed crystal structure is not necessarily that of lowest free energy of the infinite crystal, but corresponds to a minimum that can be reached by a growth process" ([149], p. 163). 

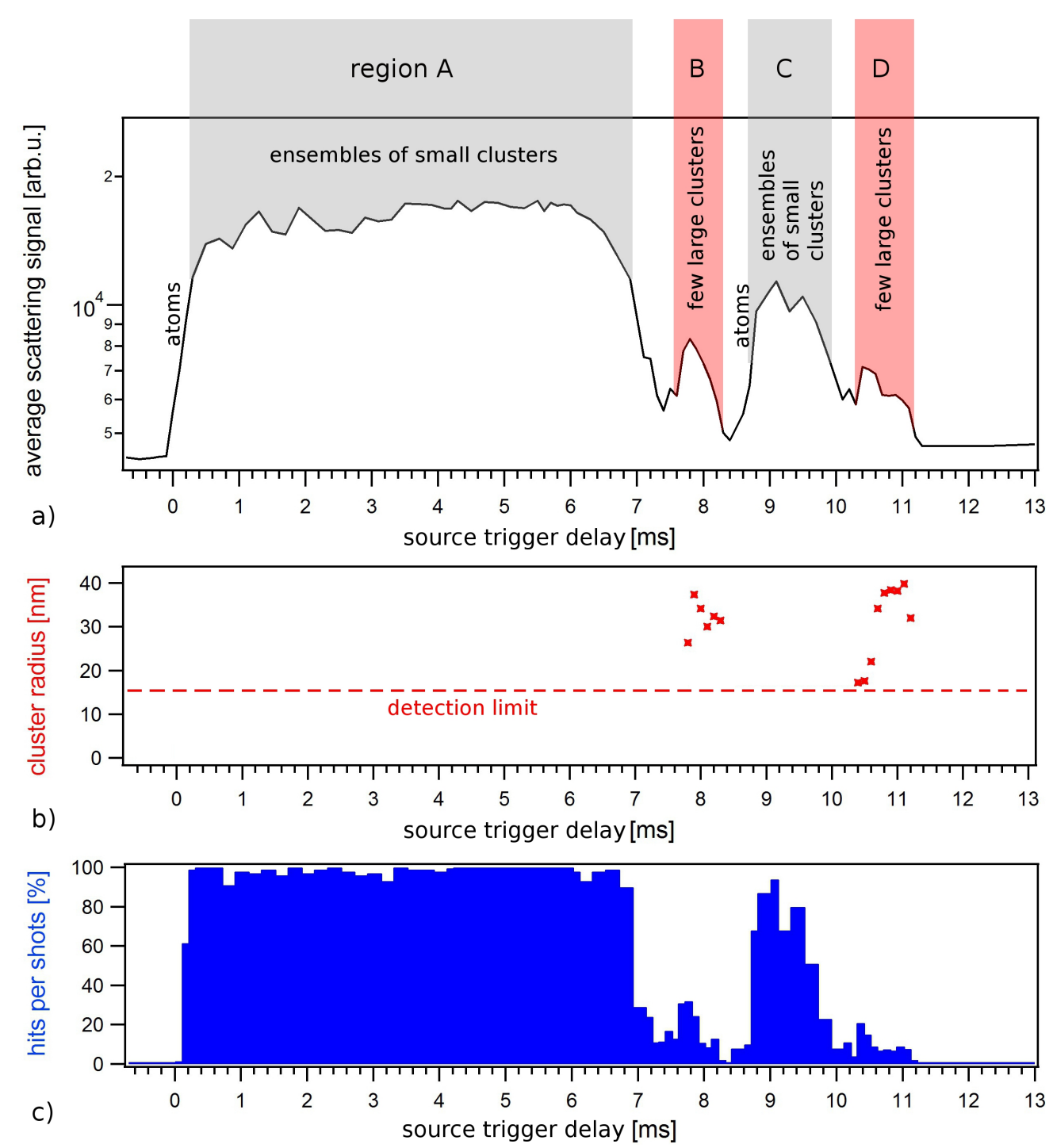

Figure 4.11: Scanning the delay between cluster source opening and FEL pulse. a) Average signal on the scattering detector over trigger delay time. b) Average cluster size of the five brightest shots for every trigger delay. Clusters with radii below $15 \mathrm{~nm}$ could not be identified. c) Hit statistics: For every delay, the number of shots with an averaged scattering signal of higher than $1.2 \cdot 10^{4}$ is divided by the number of all shots.

\subsubsection{Time structure of a pulsed jet}

In this experiment for the first time large clusters with radii up to $1 \mu \mathrm{m}$ were produced and studied in a controlled way. The highest abundance of large cluster sizes was observed, when the cluster source was triggered surprisingly early so that the snapshot of the interaction region taken by the FEL pulse should have missed the cluster pulse. Because of this finding the time structure of the cluster jet and in particular the cluster sizes as a function of source trigger delay were investigated in more detail. The time structure of the cluster pulse is discussed in this section on the basis of three delay scans of the cluster jet at different expansion conditions. Pressure and temperature were adjusted to initial conditions far below (case I), slightly below (case II) and slightly above the vapor pressure curve of xenon (case III). For comparison, also one trigger delay scan was taken for atomic gas. These data sets 


\begin{tabular}{|l|l|l|l|}
\hline & Case I & Case II & Case III \\
\hline$R$ in region $\mathrm{B}+\mathrm{D}$ & $35 \pm 5 \mathrm{~nm}$ & $155 \pm 50 \mathrm{~nm}$ & - \\
\hline$<N>$ in region $\mathrm{B}+\mathrm{D}$ & $3.1 \cdot 10^{6}$ & $2.7 \cdot 10^{8}$ & - \\
\hline$R$ from scaling laws & $8 \mathrm{~nm}$ & $14 \mathrm{~nm}$ & $24 \mathrm{~nm}$ \\
\hline$<N>$ from scaling laws & $4.0 \cdot 10^{4}$ & $2.0 \cdot 10^{5}$ & $1.1 \cdot 10^{6}$ \\
\hline
\end{tabular}

Table 4.1: Average cluster sizes of regions B and D and predictions from the scaling laws.

were actually not obtained in the beamtime 2011 but one year later with a very similar setup. Cases I, II and III will be compared to data obtained with expansion conditions considerably above the vapor pressure curve. This fourth data set (case IV) was obtained using the setup described in this thesis. The four $(p, T)$-values are marked in Fig. 4.14.

The data set of case I is displayed in Fig. 4.11. Using a nozzle with $200 \mu \mathrm{m}$ throat diameter and a half cone angle of $4^{\circ}$, clusters were produced at a stagnation pressure of 3 bar xenon and room temperature. According to the scaling laws discussed in section 3.2.2, for these expansion parameters an average cluster size of $4 \cdot 10^{4}$ atoms, i.e. $8 \mathrm{~nm}$ radius is estimated. The skimmer slit opening was set to $0.3 \mathrm{~mm}$, which does not correspond to single cluster mode, therefore ensembles of clusters were intercepted by each FEL pulse. The scattering pattern and ion spectra were measured for a fixed valve opening time of $5 \mathrm{~ms}$ and varying delays between opening of the nozzle valve and FEL pulse. In the first graph, Fig. 4.11 a, the average signal on the scattering detector is plotted over delay time. Different time domains are labeled and the characteristic features of the single shots within the respective period are described. The curve exhibits a steep rise at $t=0$ when the leading edge of the cluster pulse and the FEL arrive at the interaction region at the same time. The following plateau-like region with a width of $7 \mathrm{~ms}$ exceeds the valve opening time by $2 \mathrm{~ms}$. This plateau is further referred to as region $\mathrm{A}$. The drop at $7 \mathrm{~ms}$ is followed by a peak at $8 \mathrm{~ms}$, which is denoted as region B. Between 9 and $10 \mathrm{~ms}$ a second brighter region (region $\mathrm{C}$ ) arises, which is again followed by another peak around $11 \mathrm{~ms}$, labeled as region $\mathrm{D}$.

The development of the average cluster size for the five brightest shots of every delay is displayed in Fig. 4.11 b. The temporal evolution from measuring ensembles of clusters to single cluster mode and the repetition of this sequence can be traced in the hit statistics given in Fig. $4.11 \mathrm{c}$.

While the ion spectra at the leading edge of the cluster pulse reveal a purely atomic behavior (no $\mathrm{Xe}^{+}$, high charge states, resolved isotopic structure), region A is characterized by constant cluster-typical ion spectra in every shot and constantly bright and unstructured scattering patterns. This indicates the presence of ensembles of many clusters in the interaction region which are too small to be resolved. Cluster sizes can only be determined by Mie scattering when at least the first minimum is visible on the scattering detector, which limits the resolved cluster size in this setup at the lower limit to a radius of $15 \mathrm{~nm}$. After the main cluster pulse of region A is over, most shots reveal no signal. Nevertheless, the average scattering signal is rather high, as sometimes bigger clusters appear in region B.

For increasing delay this basic sequence is repeated with a shorter, homogeneously bright plateau of region $\mathrm{C}$ and again large single clusters in region $\mathrm{D}$.

The sequence of regions A-D (a plateau with bright scattering patterns, few large clusters about $1 \mathrm{~ms}$ after the plateau region, a second, shorter plateau and a second region with few large clusters) has turned out to be very similar for all three measurement sets I, II and III, even though the specific delay values are shifted. However, the different expansion conditions strongly change the average size of the large clusters found in regions B-D. The measured size 

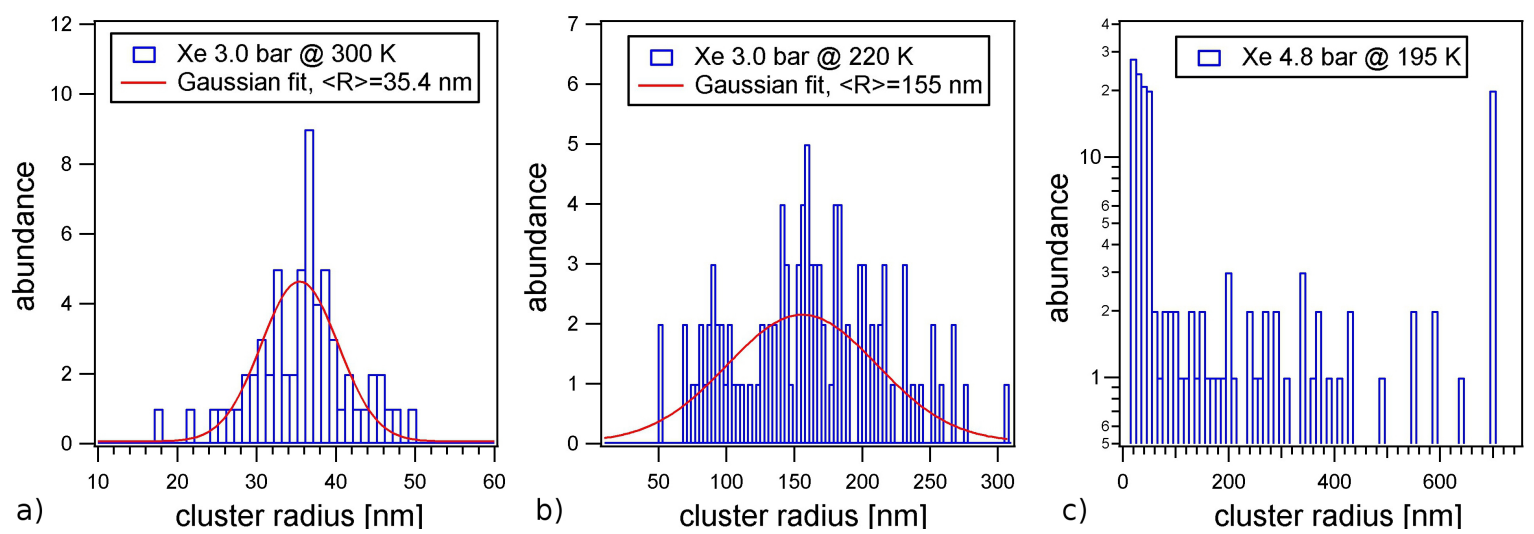

Figure 4.12: Size distributions of large clusters found after region $\mathbf{A}$ for cases I, II, and III. a) For expansion conditions of 3 bar xenon gas at $300 \mathrm{~K}$, an average size of $R=(35 \pm 5) \mathrm{nm}$ is observed in region B-D. b) In case II, at 3 bar and $220 \mathrm{~K}$, a size distribution of $R=(155 \pm 50) \mathrm{nm}$ is found for region B-D. c) For case III, 4.8 bar at $195 \mathrm{~K}$, no Gaussian distribution is found. The reason therefore might be the large amount of already resolvable clusters from the "normal" distribution. Further, 20 clusters were too big to resolve their size, and therefore set to $R=700 \mathrm{~nm}$. (Note log-scaling)

distributions of regions B-D are displayed in Fig. 4.12 for the cases I ( 3 bar at $300 \mathrm{~K}$ ) in a, II ( 3 bar at $220 \mathrm{~K})$ in b, and III ( 4.8 bar at $195 \mathrm{~K}$ ) in c. In case I and II, a Gaussian distribution has been fitted to the histograms.

The average cluster radii and numbers of particles as well as the predicted values from the scaling laws, Eq. 3.10 are summarized in table 4.1. For smallest clusters (case I) the observed averaged number of atoms per cluster in regions B-D of $3.1 \cdot 10^{6}(R=(35 \pm 5) \mathrm{nm})$ is about 2 orders of magnitude bigger than predicted from the scaling laws $\left(N=4.0 \cdot 10^{4}\right.$ atoms per cluster).

In case II, regions B-D yield an average number of atoms of $2.7 \cdot 10^{8}(R=(155 \pm 50) \mathrm{nm})$. Hence, the average number of atoms expected from the scaling laws of $N=2.0 \cdot 10^{5}$ atoms is exceeded by more than 3 orders of magnitude.

The conclusion in case III is complicated for two reasons. A strong increase towards smaller clusters is observed in Fig. $4.12 \mathrm{c}$, which presumably belongs to the "normal" size distribution of region A, because the average cluster size predicted from the scaling laws is already above the resolution limit of the experiment ( $R=24 \mathrm{~nm}, N=1.1 \cdot 10^{6}$ atoms). The peak at $700 \mathrm{~nm}$ is an artifact, because clusters with sizes larger than $R=700 \mathrm{~nm}$ can not be resolved, as the ring structures become too fine. Therefore, a value for the average cluster size of case III can not be given. The number of atoms per cluster at the upper resolution limit of $700 \mathrm{~nm}$ of $N=2.5 \cdot 10^{10}$ might serve as an orientation.

Measurements with an atomic beam as displayed in Fig. 4.13 indicate, that the two-pulse structure is a characteristic property of the valve used in the experiment. Here the integral of the averaged ion spectra for intermediate charge states of xenon $(2+$ to $6+)$ is plotted versus valve delay. Also in the ion signal of an atomic jet, a second, shorter signal increase around 9 ms can be observed. One possible explanation therefore would be a re-bounce of the sealing poppet, which causes a second, shorter opening.

The diameters of the clusters observed after both opening phases exceed the size predicted by the established scaling laws for cw-jets. Therefore the question arises, whether they would also appear in cw-jets or if their emergence is directly connected to the pulsing process. Three different scenarios are conceivable: 


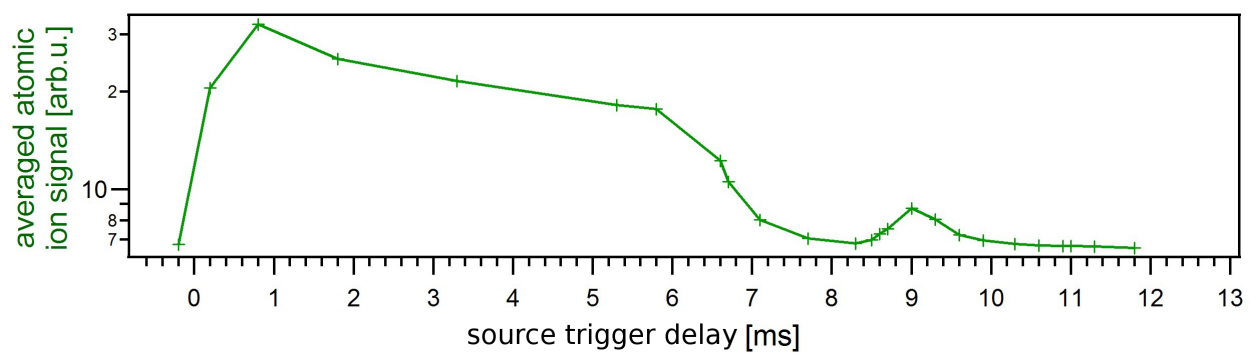

Figure 4.13: Source trigger delay scan with an atomic gas beam. The ion signal of $\mathrm{Xe}^{2+}$ to $\mathrm{Xe}^{6+}$ is integrated. The two-modal structure appears to be a characteristic feature of the valve.

- The large clusters emerge from the opening process.

- The large clusters are always produced, but in the plateau region of a pulsed source or in a cw-jet they are buried by the signal of many smaller clusters.

- They are produced during the closing of the valve.

The re-bounce of the valve and the resulting second opening, observed in Fig. 4.11 and Fig. 4.13, obviously is an artifact of this specific pulsed valve. Nevertheless it can help to interpret the time-structure of the cluster size distribution. As both plateau regions are followed by a phase of large clusters, approximately at the same distance to the respective falling edge, a generation process involving the nozzle opening becomes less probable, because of the different timing between the rising edges and the phase of large clusters.

Also the second hypothesis can be excluded for the cases I to III, because the scattering signal of large clusters would dominate the signal from even many smaller clusters. All three scans, for moderate and extreme expansion conditions have shown that no extremely large clusters appear within the plateau region before the valve closing. Thus it has to be concluded that the emergence of cluster sizes much bigger than predicted from scaling laws under the existent conditions in cases I to III, is an effect of pulsed nozzles and is associated with the closing process of the valve.

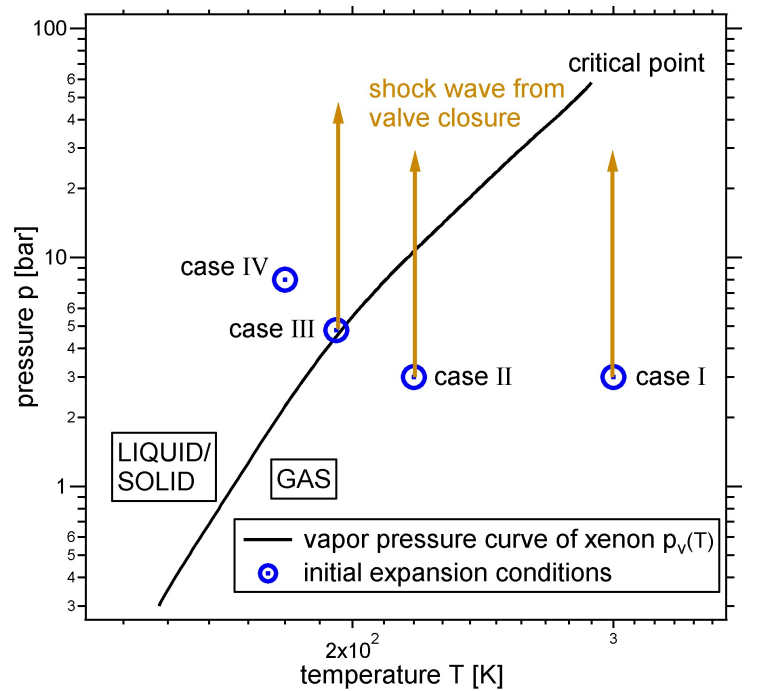

Figure 4.14: Sketch of the basic idea for explaining the emergence of large clusters in the closing of the valve.
However, in the scattering patterns taken under most extreme conditions clearly above the vapor pressure curve (case IV, 8 bar xenon at $180 \mathrm{~K}$, no scan available) clusters with radii up to the $\mu \mathrm{m}$ range are already apparent in the plateau region of the valve opening, though they remain very rare [127].

The underlying process leading to the emergence of such large clusters is not yet fully understood and subject to current discussion [152], but a first idea of the process is sketched in Fig. 4.14. While the sealing poppet is shot into the nozzle throat, a pressure shock wave might shift the initial expansion conditions towards much more extreme values, as the gas in the small volume in 


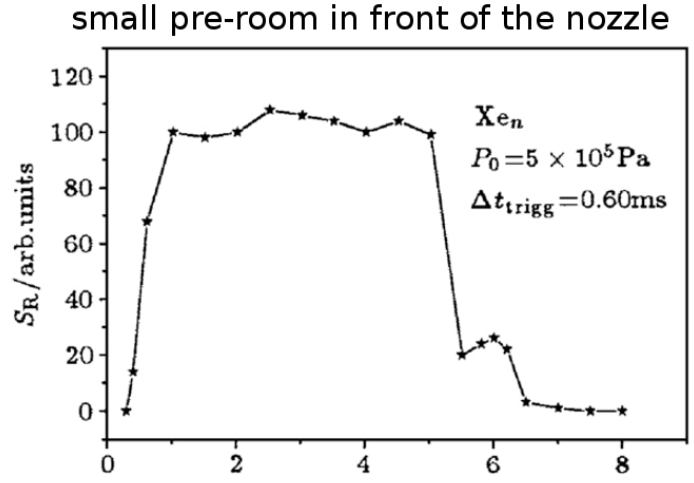

a)

Delay time/ms

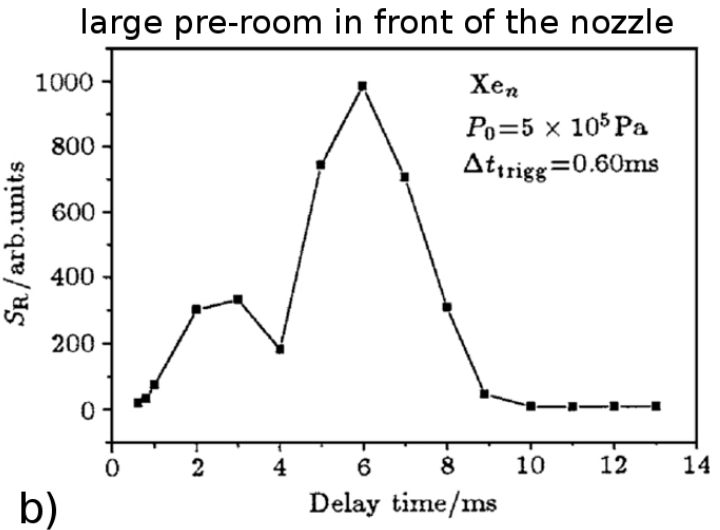

b)

Figure 4.15: Time resolved study of very short cluster pulses $(\approx 0.5 \mathrm{~ms})$ with Rayleigh scattering [153]. The scattered light as a function of trigger delay reveals a two-plateau structure of the scattered light. The second feature becomes more prominent with a larger "pre-room" in front of the nozzle.

front of the nozzle throat is compressed. In cases II and III, initial expansion conditions far above the vapor pressure curve could reach an expansion out of the liquid phase, which is already apparent for the initial conditions of case IV.

These findings, that bigger clusters appear after the valve closing, are also in accordance with a time resolved study of very short cluster pulses with Rayleigh scattering from Shao-Hui et.al [153]. They observed a two-plateau structure of the scattered light which increased with a larger room in front of the nozzle, see Fig. $4.15 \mathrm{a}$ and b. Their interpretation is based on the assumption of dimers generated in this pre-room, which subsequently grow to larger sizes. However, as the gas temperature in front of the nozzle throat is still high, condensation in the pre-room seems to be rather unlikely.

Further, the emergence of very large clusters appears to be related to a velocity dispersion of particles in the jet. The delay scans of cases I-III clearly show, that the large clusters do not emerge in the falling edge but delayed to the time of the closing of the valve (cf. Fig. 4.11). Apparently, with more extreme expansion conditions the delayed onset shifts to even later times. In the literature, the observation of clusters being slower than the uncondensed gas, and bigger clusters slower than smaller ones, is referred to as velocity slip [154]. In this concept, the over-proportional growth can be attributed to the slowest clusters, which grow by picking up faster ones. They are accelerated by the collisions, but the mass of the particles increases stronger than their velocity.

A more technical outcome of this time-resolved analysis of the cluster jet is, that the opening conditions in future experiments can be further optimized. The steep rise at the beginning of the plateau in Fig. 4.11 indicates that less than $1 \mathrm{~ms}$ is needed to reach stable expansion conditions within the "normal" expansion regime of region A, where the size distributions follow the scaling laws Eq. 3.10 [144]. In imaging experiments, where single large clusters are required, even shorter opening times can lead to optimal conditions shortly after the valve closing. This is of particular importance when the gas load on the vacuum pumps limits the repetition rate or very expensive gases, such as xenon are used.

In addition, the discovery and the phenomenological characterization of the occurrence of extremely large clusters after the pulse gives access to a new regime of studies of the lasercluster interaction. In the remaining part of this chapter, power density dependent effects in the ion spectra and scattering patterns of single very large clusters will be investigated. 

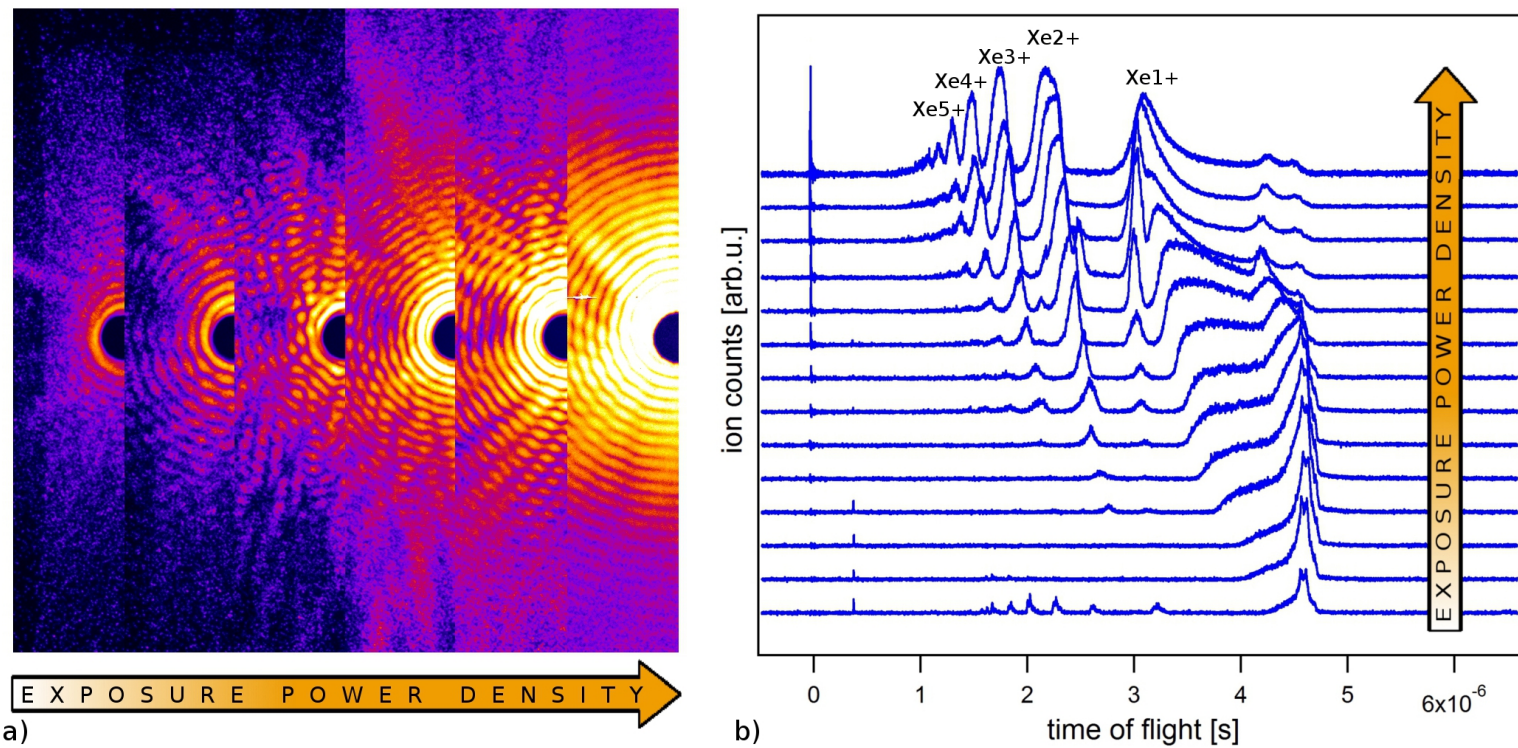

Figure 4.16: Selection of scattering patterns and ion spectra of clusters with radius $R=400 \pm 50 \mathrm{~nm}$. The clusters are intercepted by the XUV pulse at different positions within the focus profile and therefore exposed to different power densities. The ion spectra are sorted for increasing exposure power density (epd).

\subsection{Ejection of surface ions from a quasi-neutral nanoplasma}

In this section, the plasma dynamics of single large xenon clusters exposed to different XUV power densities are investigated by means of ion spectroscopy. The ion spectra from single clusters are sorted by their size and the power density they were exposed to, yielding information on the ionization and recombination processes. The ion kinetic energy distributions extracted from the time-of-flight spectra give insight in charge distributions and screening effects in the clusters and reveal the competition between recombination and expansion dynamics in a highly dense nanoplasma.

In the first section 4.4.1, the development of the ion spectra with increasing power density of the XUV pulse, sorted for clusters with $R=400 \mathrm{~nm}$, is described phenomenologically. Initial kinetic energies of the ions are modeled in a SIMION-simulation of the spectrometer geometries, which is described in detail in section 4.4.2. The key results from the analysis of the ion kinetic energies for cluster radii ranging from 180 to $600 \mathrm{~nm}$ are presented in section 4.4.3. The kinetic energies of the ions are narrowly distributed around a central kinetic energy, which is directly proportional to their respective charge state.

These findings are discussed in section 4.4.4 on the basis of previous experimental and theoretical results about the characteristic properties and dynamics of xenon clusters in $90 \mathrm{eV}$ pulses. The data indicate that the ions are ejected from the surface of a mostly neutral, dense plasma, which recombines completely in the subsequent expansion process. Considering simple electrostatic models of the initial state, an effective charge on the clusters can be calculated. Its size dependent development is investigated and discussed in section 4.4.5. 


\subsubsection{The concept of exposure power density (epd)}

With the information from the scattering patterns a distinct size range of clusters can be selected. Subsequently the respective hits are sorted for exposure power density (epd), which denotes the laser intensity at the respective focal position of each cluster. The concept of "exposure power density" is introduced, to avoid confusion with the term "intensity", which is also used for detector signals, such as scattered light.

For a selected cluster size, a direct relation between the scattering signal of the hits gathered in such a data set and their epd values can be found if two assumptions are made:

1. The scattered signal increases proportionally to the power density of the incoming light.

2. The data set contains enough hits to map the whole focus profile.

In this case, the hit with the most intense scattering pattern can be assigned to the maximal power density of the focus. The remaining hits are assigned in respect to this value by the ratios of their scattering signal to the maximal one. The validity of both assumptions has to be considered carefully, as discussed in more detail in section 4.4.3. However, even without attempting an assignment of absolute epd values, the scattering signal can be used for simply sorting the hits within one data set for increasing epd.

An illustrating example for the derived epd scans is presented in Fig.4.16. Here, selected scattering patterns and ion spectra from the cluster size with highest abundance of $R \approx 400$ $\mathrm{nm}$, are sorted for increasing epd. Based on this scan, an introduction into the structure of the ion spectra and their characteristic development shall be given in this paragraph.

In the ion spectra, the count rate of the detector is plotted over flight time of the ions. For orientation, the ion charge states in the most intense hit in Fig. 4.16 are labeled up to $\mathrm{Xe}^{5+}$. The most important structures of the ion spectra developing with epd, are from short to long flight times:

- The ion spectra exhibit a sharp peak at $\mathrm{t}=0$ except for the weakest hit. This peak arises from elastically scattered light of the cluster in the interaction region, which increases with epd.

- In medium to most intense hits, between 1 and $3 \mu$ s relatively narrow peaks appear, which belong to charge states of $\mathrm{Xe}^{2+}$ and higher. With increasing epd they grow and shift towards shorter flight times due to increasing initial kinetic energy of the ions.

- The $\mathrm{Xe}^{1+}$ signal behaves somewhat differently. The maximum is also shifting towards higher kinetic energies, from about $4.6 \mu \mathrm{s}$ to $3.2 \mu \mathrm{s}$ in terms of time-of-flight. But the count rate at longer time-of-flight does not vanish and the $\mathrm{Xe}^{1+}$ peak does not reach the narrow, Gaussian shape of the higher charge states.

\subsubsection{SIMION simulation of the bipolar time-of-flight spectrometer}

In order to attribute ion kinetic energies to the measured times-of-flight, the trajectories of ions for different initial kinetic energies can be modeled in a simulation of the tof spectrometer. Further, it is important to understand the transmission function of the bipolar tof spectrometer, which was used in the experiment (see also section 3.2.4). An example 


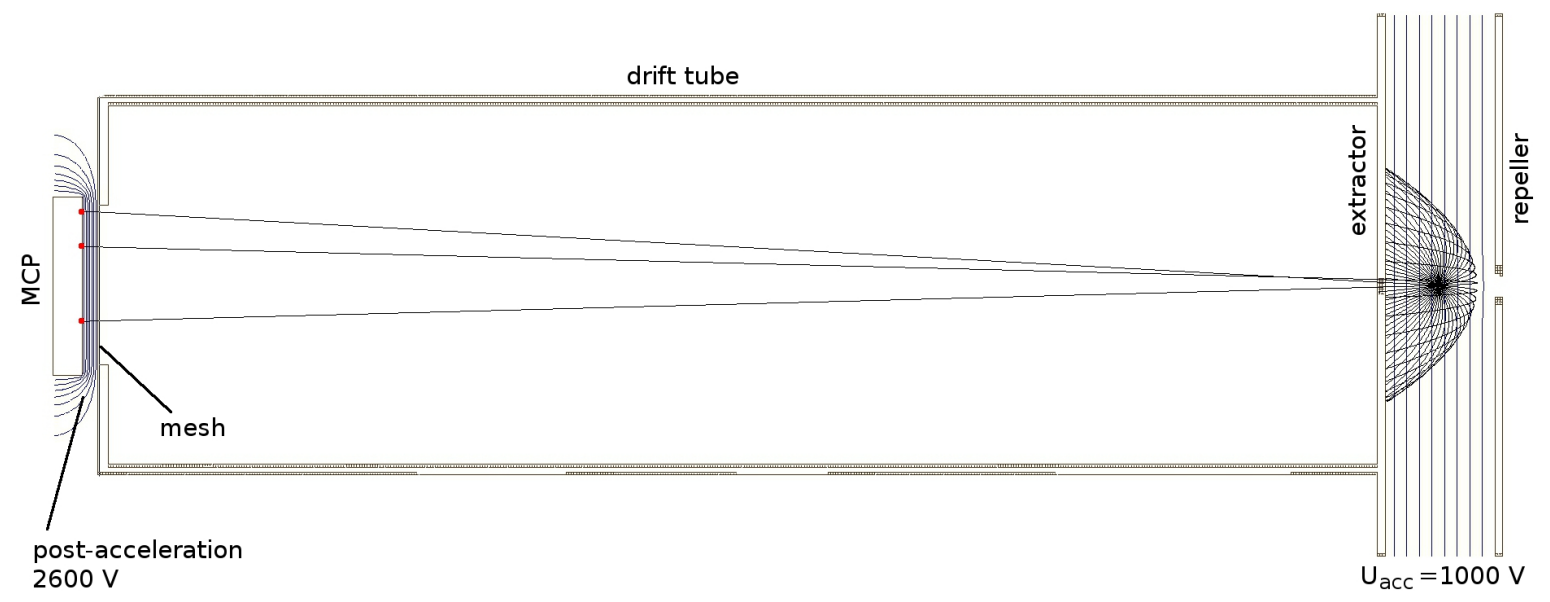

Figure 4.17: Sectional view of the model of the bipolar tof spectrometer in SIMION. The equipotential lines of the electric field strength are depicted in blue. Simulated trajectories of ${ }^{132} \mathrm{Xe}^{2+}$ ions with $E_{k i n}=700 \mathrm{eV}$ reveal a decreased transmission for high kinetic energies. Only three of 60 ions equally distributed over $360^{\circ}$ reach the MCP. Red points indicate that the respective times-of-flight and energies were recorded by SIMION.

of the influence of the spectrometer transmission can be found in the rather strong peaks in Fig. 4.16 at a flight time around $3.3 \mu \mathrm{s}$, which seem to shift slightly to longer times-offlight. These are back-peaks which arise from ions (here of $\mathrm{Xe}^{2+}$ ) ejected away from the detector and later reversed by the spectrometer field. Under some circumstances these ions experience an unexpectedly high transmission in this specific spectrometer. Based on well characterized transmission properties, such spectrometer artifacts can be distinguished from physical effects.

The simulation of the spectra was done using SIMION [155, 156], a commercial software which enables modeling electrostatic fields and trajectories of charged particles in different electrode geometries. Via stl-format the 3D CAD-geometry of the bipolar tof can be loaded into SIMION. In Fig. 4.17 a sectional view of the spectrometer in SIMION with equipotential lines in blue is displayed. Corresponding to the values in the experiment the potential of the repeller-plate is set to $1000 \mathrm{~V}$, the extractor-plate and the drift tube are grounded. A short post-acceleration towards the MCP at $-2600 \mathrm{~V}$ has only a small influence on the flight time, but modeling the mesh at the end of the drift tube is important to prevent the field from reaching into the drift tube.

The transit of ions through the spectrometer was analyzed in the following way. In a first step, the accurate $y$-coordinate of the interaction region was determined by matching the simulated flight times of different xenon charge states without initial kinetic energy to the experimentally obtained spectra of atomic xenon gas.

Subsequently, flight times for ions of different charge states and initial kinetic energies were obtained from the simulation. In Fig. 4.17, the black lines show trajectories of doubly charged ${ }^{132} \mathrm{Xe}$ with an initial kinetic energy of $700 \mathrm{eV}$ and different starting angles. For clarity of the illustration, here the initial directions of the ions are fixed to the image plane and distributed in steps of $6^{\circ}$. Only three of 60 ions reach the MCP, red points indicate that their initial energies and times-of-flight through the spectrometer were recorded by SIMION.

The results of the simulated time-to-energy conversion function for ions in the bipolar tof directed towards the MCP are presented in Fig. 4.18. The time-of-flight of ions with an 

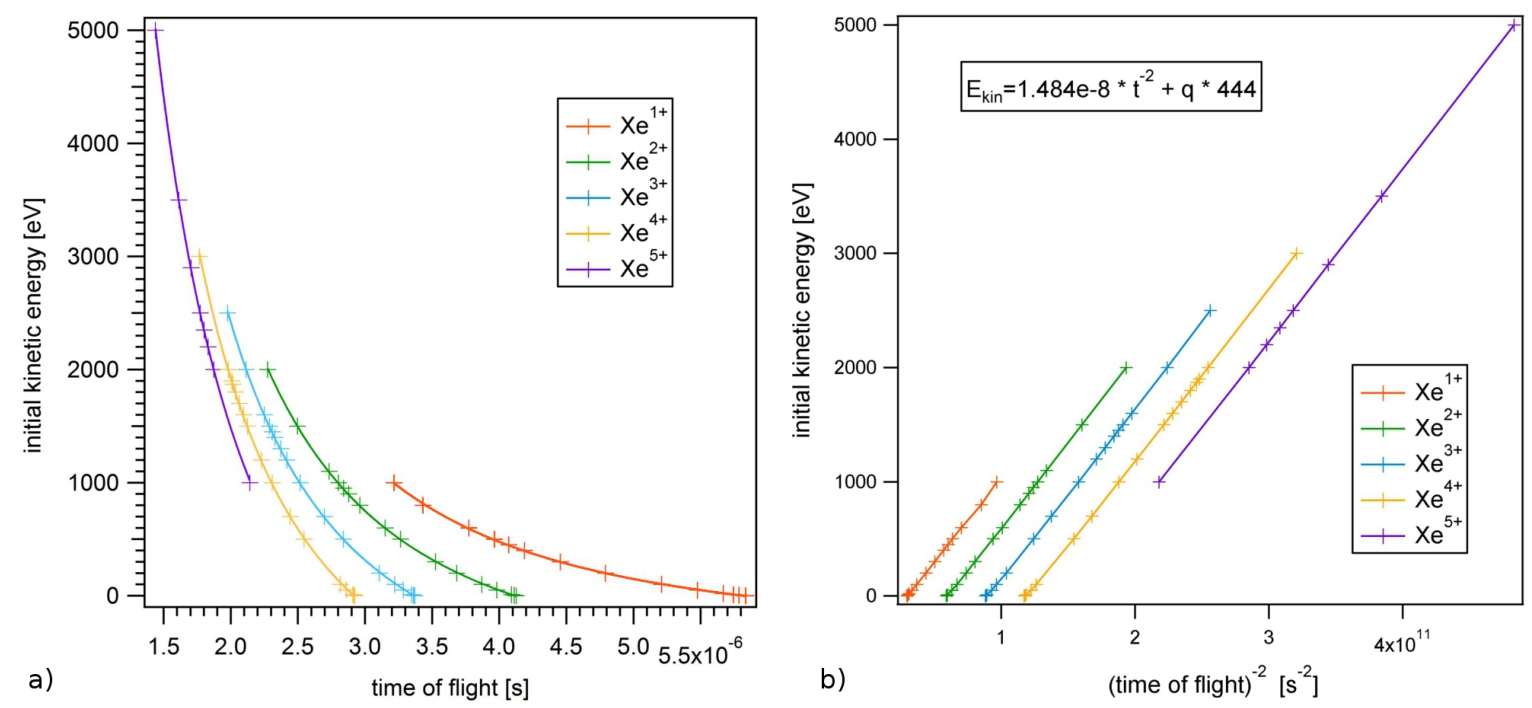

Figure 4.18: a) Time-to-energy conversion function for xenon charge states up to $5+$. b) The initial energy as a function of $t^{-2}$ decreases linearly, following the linear equation $E_{\text {initial }}=1.48 \cdot 10^{-8} \cdot t^{-2}+$ $q \cdot 444$.

isotropic angular distribution of starting directions in $2 \pi$ towards the detector and distinct initial kinetic energies was simulated. Only ${ }^{132} \mathrm{Xe}$ was considered and other isotopes of xenon were neglected. In Fig. 4.18 a the conversion functions of time-of-flight into kinetic energy for charge states up to $5+$ are presented. As displayed in Fig. 4.18 b, the initial kinetic energy is a quadratic function of the reciprocal time-of-flight. The conversion functions of all charge states follow the linear equation $E_{\text {initial }}=1.48 \cdot 10^{-8} \cdot x+q \cdot 444$ with $x=t^{-2}$.

Further, the transmission function of the bipolar tof spectrometer was determined. The trajectories were simulated for an isotropic angular distribution of starting directions in $4 \pi$. Count rates were collected for all ions which reach the detector (cf. red points in Fig. 4.17). In Fig. 4.19 a the transmission function of the spectrometer, defined as the ratio of launched ions to those impacting the MCP is plotted for all charge states up to $8+$. The transmission changes with charge state and initial kinetic energy. For a given $E_{\text {initial }}$, the transmission is lowest for singly charged ions, as the spectrometer field will influence the trajectories stronger for higher $q$. For all curves, at an initial energy of $\approx 0.45 \cdot U_{a c c} \cdot q$ the linearly decreasing curves in the double-logarithmic plot of Fig. $4.19 \mathrm{~b}$ are disrupted by sharp peaks, where the transmission is enhanced by a factor of 2 to 6 . A closer analysis of the trajectories reveals that the enhancement of the back-peaks is due to ions which start in a direction away from the detector with an initial energy almost sufficient to reach the repeller plate before they are reversed by the spectrometer field.

The resonant-like behavior of the transmission which is found in the simulation can be also observed in the measured spectra. The back-peaks exhibit several special characteristics, as derived from the analysis of trajectories and times-of-flight:

- Only for smallest kinetic energies the back-peaks arrive at longer flight-times than the zero-kinetic atomic peaks. For $E_{k i n}>10 \mathrm{eV}$, the atomic ions will enter the drift tube first, but the back-peaks will overrun the slower atomic ions.

- With further rising $E_{k i n}$, the back-peaks do not shift monotonously towards shorter flight-time as the forward peaks do. Rather, the ions of a whole energy range are time- 

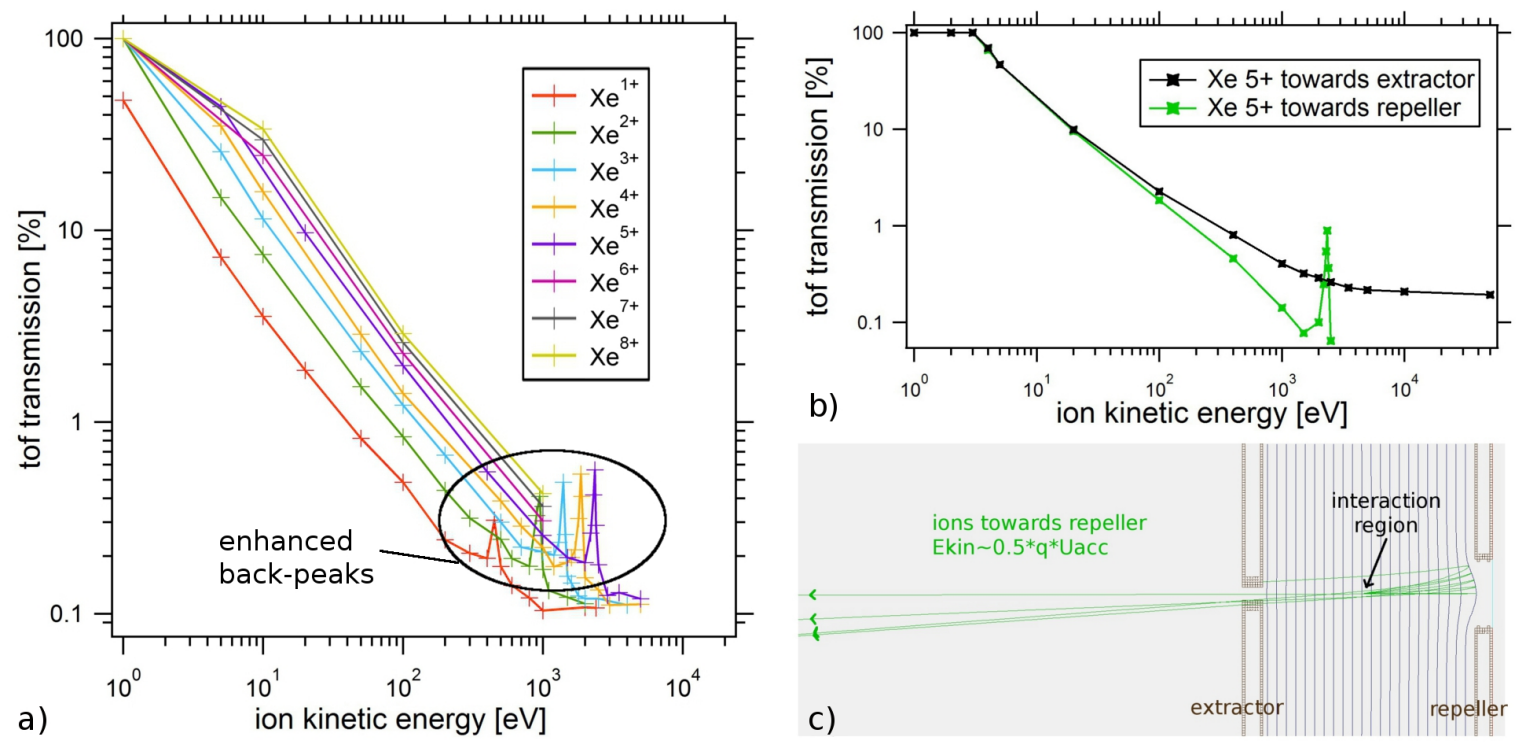

Figure 4.19: a) Transmission functions for $\mathrm{Xe}^{1+}$ up to $\mathrm{Xe}^{8+}$. The transmission of ions decreases strongly with increasing initial kinetic energy. From a distinct energy on, only the solid angle which is defined by the aperture in the extractor plate will be transmitted and the transmission stays constant. Before constant transmission is reached, a resonant-like enhancement can be observed.

b) The enhanced transmission is due to back-peaks with an initial kinetic energy of $\approx 0.45 \cdot U_{a c c} \cdot q$ as indicated by the transmission functions of $\mathrm{Xe}^{5+}$ ions starting towards and away from the detector. c) These ions come close to the repeller plate before they are reversed in the spectrometer field. Due to a hole in the repeller electrode the field is distorted and causes a lens effect. Therefore, a high number of ions are focussed through the extractor aperture.

focussed on a distinct tof-value. As a consequence, even though they result from high kinetic ions with some $100 \mathrm{eV}$ broad distributions, some back-peaks even exhibit the xenon isotope structure.

- At a distinct kinetic energy for each charge state of $E_{\text {initial }} \approx 0.45 \cdot U_{a c c} \cdot q$, the backpeaks experience a "resonant" transmission, due to a lens-effect of the center hole in the repeller plate.

In Fig. $4.19 \mathrm{~b}$ and c this situation is further investigated. Fig. $4.19 \mathrm{~b}$ displays separately plotted transmissions of $\mathrm{Xe}^{5+}$ ions directed towards and away from detector revealing the enhanced transmission only for the back-ejected ions. The trajectories of those ions can be seen in Fig. 4.19 c. Close to the repeller electrode the field is distorted by the opening in the plate. This distortion produces a lens-like effect, resulting in a much higher number of ions being focussed through the aperture of the extractor plate.

For even higher initial kinetic energies than the respective resonant energy, the back-peaks hit the repeller or leave the spectrometer through the repeller aperture, thus they do not play a role in the spectra anymore. Also for fast ions starting in forward direction, the spectrometer field has less influence and the transmission becomes dominated by the geometric solid angle of the aperture in the extractor plate. Thus, all transmission curves approach a constant value of $\approx 0.15 \%$ for high kinetic energies.

With the conversion function in Fig. 4.18 and the transmission function of Fig. 4.19, now the tools are at hand to understand and explore the measured spectra in detail. The options for further analysis are discussed on the example of the hit with the fourth-brightest epd value from the data set of clusters with $R=400 \mathrm{~nm}$ as displayed in Fig. 4.16. This spectrum 


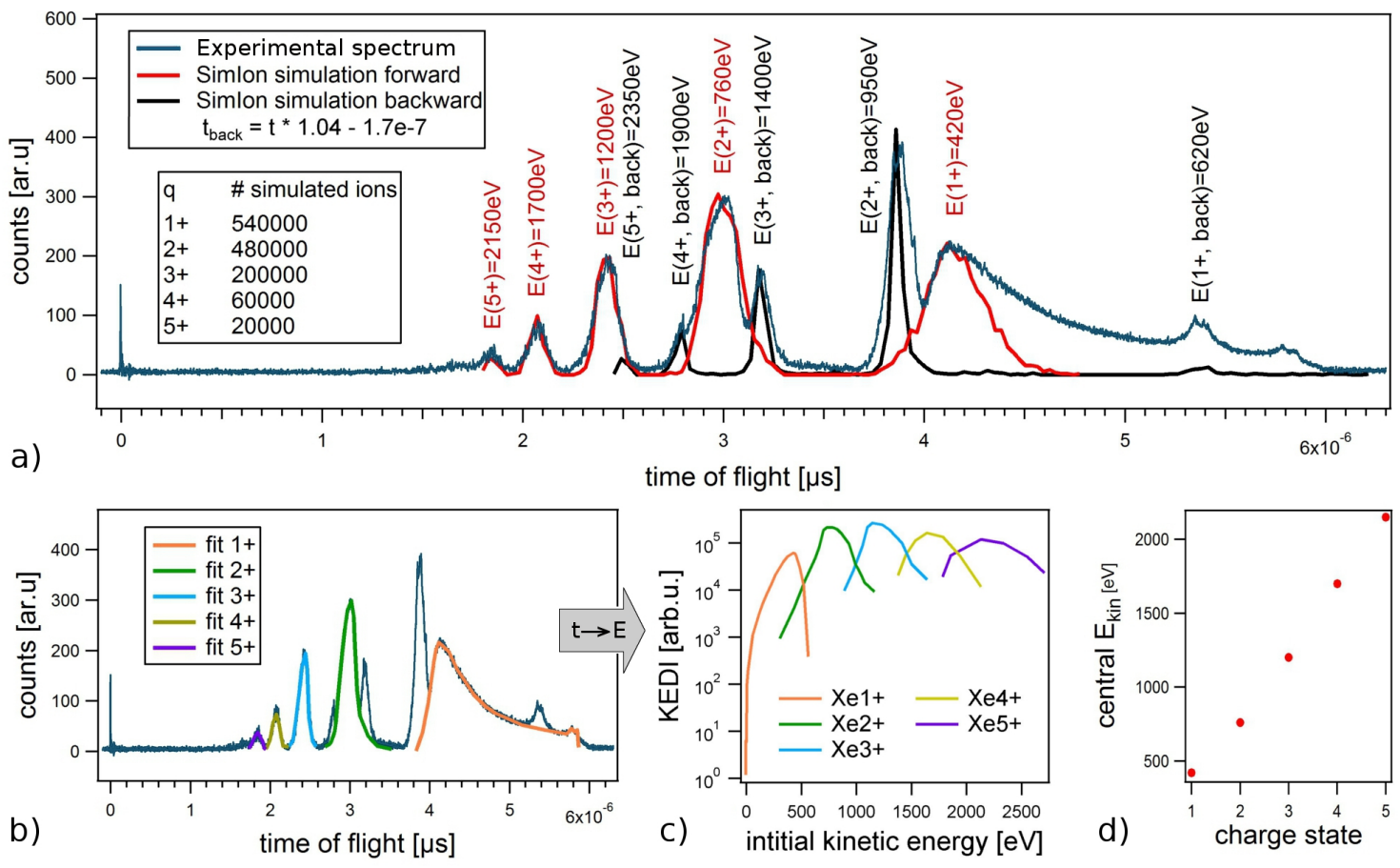

Figure 4.20: Ion spectrum of $400 \mathrm{~nm}$ cluster with high back-peak contribution (blue curve) analyzed in SIMION: a) Simulated ion distributions match the the experimental ion spectrum. Measured backpeaks show resonant behavior at lower kinetic energies as predicted from the simulation, and their flight time is shifted towards larger values. For the simulations, Gaussian distributions are assumed. b) The peaks differ from Gaussian shape. Therefore, the accurate distributions of the forward peaks for each charge state are derived by polygon-fits. d) By applying conversion and transmission function of each charge state to the polygon-fits, a transmission-corrected energy distribution of ions (KEDI) is generated. d) The central kinetic energy is a linear function of the charge state. The central energies are derived from the SIMION simulation (red curve in a).

is chosen because it exhibits a strong back-peak contribution. The measured ion spectrum, simulated curves and derived results are displayed in Fig. 4.20.

In Fig. 4.20 a the peaks in the ion spectra are classified by direct modeling. For the simulation, a large number of ${ }^{132} \mathrm{Xe}$-ions with charge states from $1+$ to $5+$ were generated and propagated through the spectrometer. For every $q$, a single Gaussian distribution of particles was assumed with central energy $E_{k i n}(q)$ and widths of $200-300 \mathrm{eV}$, randomly directed in the hemisphere towards the detector. The energies and particle numbers were varied to obtain a good match with the forward peaks of the experimental spectrum. The red curve in Fig. 4.20 a results from the propagation of $1.3 \cdot 10^{6}$ ions, distributed over $q$ as displayed in the inset of Fig. 4.20 a. For charge states $q>2$ a satisfying fit can be reached, considering the noise level of the experimental spectrum. However, for $\mathrm{Xe}^{1+}$ only the high kinetic part of the feature is matched well by the Gaussian distribution of ions.

It should be noted, that the calculated spectrum for ions directed in the hemisphere away from the detector, i.e. the back-peaks, could not achieve a reasonable match to the experimental spectrum if the conditions found for the forward peaks were used. Apparently, only the appearance and the main features of the back-peaks can be explained by the SIMION simulation, the trajectories are not reproduced accurately.

Only by artificially increasing the initial energies of all charge states by about $200 \mathrm{eV}$, the transmission behavior of the back-peaks in this spectrum, i.e. the abundance compared to 
each other and to the forward peaks, could be matched. In addition, the measured back-peaks are found in the spectrum at longer flight times than one would expect from the simulation. Therefore, the black curve in Fig. $4.20 \mathrm{a}$ had to be stretched and shifted to fit the measured spectrum. The adjustment is specified in the legend of Fig. 4.20 a.

A discrepancy between measured and simulated back-peaks has been observed earlier [137]. Two main reasons can be accounted for this, both derive from the general deficiency of the simulation. First, to explain the enhancement of the back-peaks, the local geometry of the repeller plate becomes important which can not be fully accounted for in the simulation. In reality, the repeller plate is made from flexible $\mu$-metal and is slightly distorted, which might also lead to a field distortion close to the aperture. Second and even more important, the evolving local charge distribution in the interaction region from the disintegrating cluster can not be accounted for in the SIMION simulation which only propagates a single ion in a static field at a time. Especially the backward directed ions are influenced by space charge in the interaction region as their trajectory passes the initial cluster position closely.

However, the simulation is certainly capable of identifying the contributions of the back-peaks in the spectrum. Then, as a next step, the accurate shape of the forward peaks from the measured ion distributions can be fitted. Fig. 4.20 b displays the manually extracted curves of the forward directed distributions for charge states up to $\mathrm{Xe}^{5+}$ by means of simple polygonfits. The conversion of time-of-flight into initial kinetic energies of the ions follows the above derived relationship $E_{\text {initial }}=a \cdot t^{-2}+q \cdot b$. Further, the ion signal per time interval $I(t)$ has to be converted into ion signal per energy interval $I(E)$ via

$$
\begin{aligned}
\frac{d I}{d E} & =\frac{d I}{d t} \frac{d t}{d E} \mid \cdot d E^{2} \\
\Leftrightarrow \quad d I(E) \cdot d E & =d I(t) \cdot d t \cdot \frac{d E}{d t} \\
\text { with } \quad \frac{d E}{d t} & =-\frac{a}{2} \cdot t^{-3},
\end{aligned}
$$

by accounting for the factor $t^{-3}$. Together with the transmission functions for each charge state, the kinetic energy distribution of ions (KEDI) as the transmission corrected ion signal per initial kinetic energy can be directly deduced from the polygon fits of the different charge states. The result is presented in Fig. $4.20 \mathrm{c}$. It is important to note that the shape of the $\mathrm{Xe}^{1+}$ peak is as sharp as the other charge states. The observation of a different behavior of the singly charged ions stated above has therefore been revealed as a combined transmission and time-to-energy conversion effect.

The KEDI derived from ion spectra or calculated in simulations is often used in related publications, for example in [96] (see Fig. 4.2, section 4.1). The KEDI in Fig. 4.20 c and important implications for the underlying expansion process and the role of recombination in the cluster plasma will be discussed in detail in section 4.4.4.

One important feature of the determined kinetic energies of the forward ions is the purely linear dependency with charge state, as shown in Fig. 4.19 d. This linearity is an important characteristic of the current single cluster data and deviates from earlier findings in ensembles of smaller clusters [94]. Therefore, in the next section, size-sorted epd scans for radii of 80 to $600 \mathrm{~nm}$ are compared with respect to their dependence on increasing exposure power density. From the ion spectra the central kinetic energy will be extracted and analyzed as a function of charge states and cluster size. 


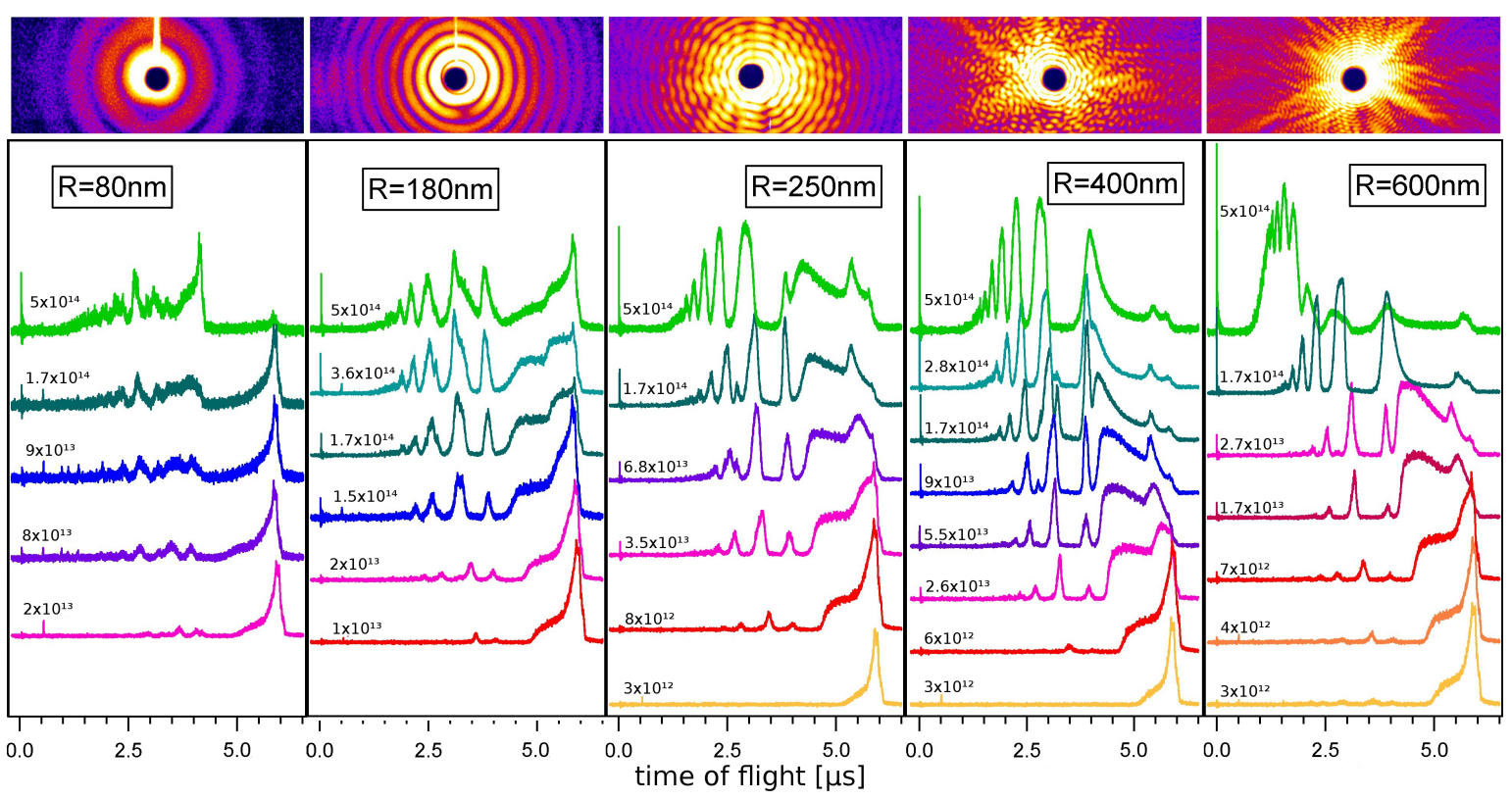

Figure 4.21: Size sorted ion spectra from single xenon clusters with 80 to $600 \mathrm{~nm}$ radius. The spectra are sorted from bottom to top for increasing epd, the indicated values are in $\mathrm{W} / \mathrm{cm}^{2}$. The assignment of epd values is described in the text. Further, as a guide for the eye comparable epd values are indicated by colors of the spectra. This color code is used consistently in the subsequent analysis.

\subsubsection{Size dependent effects in kinetic energy distributions}

In Fig. 4.21 epd scans for different cluster sizes with radii of $R=(80 \pm 10) \mathrm{nm},(180 \pm 30) \mathrm{nm}$, $(250 \pm 40) \mathrm{nm},(400 \pm 50) \mathrm{nm}$, and $(600 \pm 50) \mathrm{nm}$ are presented. In the case of the smallest cluster size, $R=80 \mathrm{~nm}$, the number of atoms per cluster amounts to $4 \cdot 10^{7}$. For the larger sizes accordingly $2 \cdot 10^{8}, 6 \cdot 10^{8}, 5 \cdot 10^{9}$, and $2 \cdot 10^{10}$ atoms per cluster are reached.

As described above, all ion spectra within one size range are sorted for exposure power density. It is important to note that the signal from the scattering detector can be used for sorting for increasing epd, but the assignment of absolute epd values is somehow problematic. One aspect is, that the scattering patterns clearly exhibit intensity dependent changes (cf. next section 4.5). Therefore, a linear dependence between scattered to incoming intensity is not necessarily valid in the case of xenon clusters at $90 \mathrm{eV}$ photon energy. Even more important, the scattering detector is not guaranteed to have a linear response for high scattering intensities, as discussed in more detail in chapter 4.5.1 and appendix A. As suggested in [104], the light peaks in the ion spectra, which constitute the amount of light under an observation angle of $90^{\circ}$, can also be used for an assignment of the exposure power density. However, the scattered light has its minimum around $90^{\circ}$ (cf. section 2.1.3), thus contributions from fluorescent light might become important. Further errors in the assignment might be caused by slightly different cluster positions under the tof aperture resulting in a different amount of detected light.

Further, in order to assign absolute epd values to the size-selected hits presented in the panels of Fig. 4.21, the assumption has to be made, that the data sets for each cluster size are large enough to map the whole focus profile. However, only the fourth panel with ion spectra from clusters of $400 \mathrm{~nm}$ radius, which has been already presented in section 4.4.1, displays a representative selection out of about 100 hits of similarly sized clusters. In contrast, the statistics for all other cluster sizes are low and the epd scans in Fig. 4.21 already contain most available hits from the respective data sets. 
In view of the considered uncertainties, the absolute epd values presented as overview in Fig. 4.22 and indicated in Fig. 4.21 can only serve as a guide. The most intense hits are assigned to the maximal focal power density of $5 \cdot 10^{14} \frac{\mathrm{W}}{\mathrm{cm}^{2}}$ which has been derived in sec-

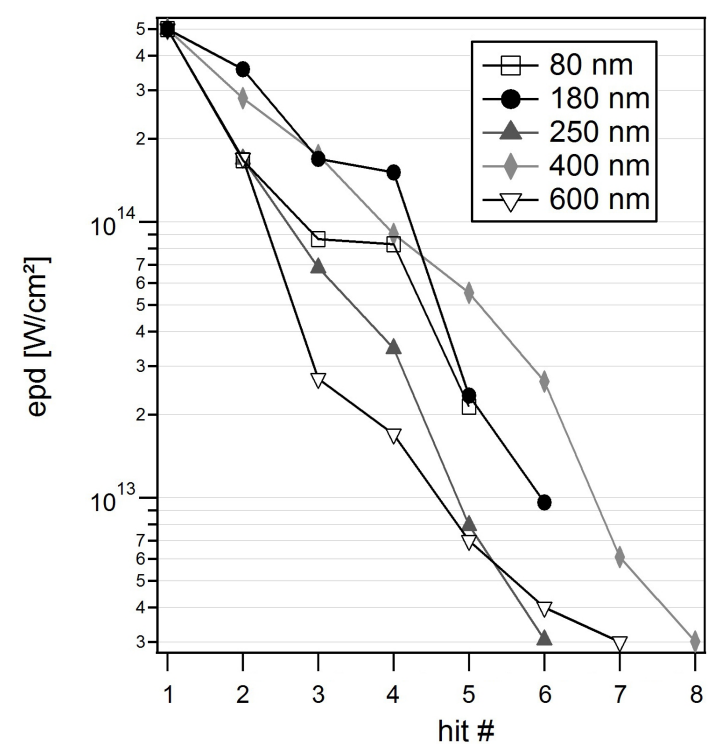

Figure 4.22: Assignment of epd values to the size sorted ion spectra from most intense to weakest hit. tion 3.2.4 from the xenon gas spectra. The epd values of the weaker hits are subsequently linearly scaled to the maximal-epd hit according to their light-peaks in the tof spectra.

This epd assignment is also indicated in Fig. 4.21 by using identical colors for spectra with comparable epd. This color code will be used consistently in the subsequent analysis. Hits with low epd without identifiable light peak in the tof spectra, as for example the weakest hit in Fig. 4.16, were not considered for this analysis, even though their size could be extracted from the scattering patterns.

As a first step, the spectra in Fig. 4.21 can be addressed in a qualitative approach of phenomenological description and comparison. The most obvious effect observed in Fig. 4.21 with increasing cluster size is the stronger increase of the ion kinetic energy. This manifests itself in the peaks shifting towards shorter flight times. The emergence of higher charge states exhibits a quite similar behavior for comparing similar epd values from $\mathrm{R}=180 \mathrm{~nm}$ to $600 \mathrm{~nm}$. For example the pink spectra with approximately $3 \cdot 10^{13} \mathrm{~W} / \mathrm{cm}^{2}$ reveal an observable contribution of $\mathrm{Xe}^{4+}$ and the dark-green curves at $1.7 \cdot 10^{14} \mathrm{~W} / \mathrm{cm}^{2}$ exhibits charge states up to $\mathrm{Xe}^{5+}$. However, as the kinetic energies of the ions increase with cluster size, the spectrometer transmission has to be considered. This effect can be observed best in the light-green spectra at an epd of $5 \cdot 10^{14} \mathrm{~W} / \mathrm{cm}^{2}$. Although the highest charge states appear to be more prominent for larger clusters, this is mainly caused by the combination of large clusters producing a stronger ion signal and the fact, that their kinetic energy is already in the range of constant transmission.

An actual qualitative change in shape and abundance of high charge states seems to appear towards small clusters $(R=180,80 \mathrm{~nm})$, especially for high epd values. In these spectra, the peaks have a more trigonal shape, in particular compared to the Gaussian shape at larger clusters. In the case of the smallest cluster size displayed $(R=80 \mathrm{~nm})$, the $\mathrm{Xe}^{1+}$ peak vanishes for highest epd instead of shifting towards higher kinetic energy. As the transmission of low kinetic ions in the spectrometer is high, this disappearance together with the increase of $\mathrm{Xe}^{2+}$ in the uppermost spectrum of the first panel indicates an actual redistribution of charge states towards higher values.

The fundamental difficulty with smaller clusters with radii of $80 \mathrm{~nm}$ and below, lies in the decreasing quality of the ion signal in single particle mode. For one, the signal to noise ratio of the ion spectra becomes worse as a smaller number of particles is detected. Secondly, very small clusters which have no measurable contribution to the scattering signal, as well as the atomic signal from the uncondensed part of the cluster beam, have an increasing influence in the ion spectra. Therefore the smallest clusters presented here have a radius of $80 \mathrm{~nm}$, cluster sizes with radii of $25-70 \mathrm{~nm}$ could not be analyzed in single shot. 


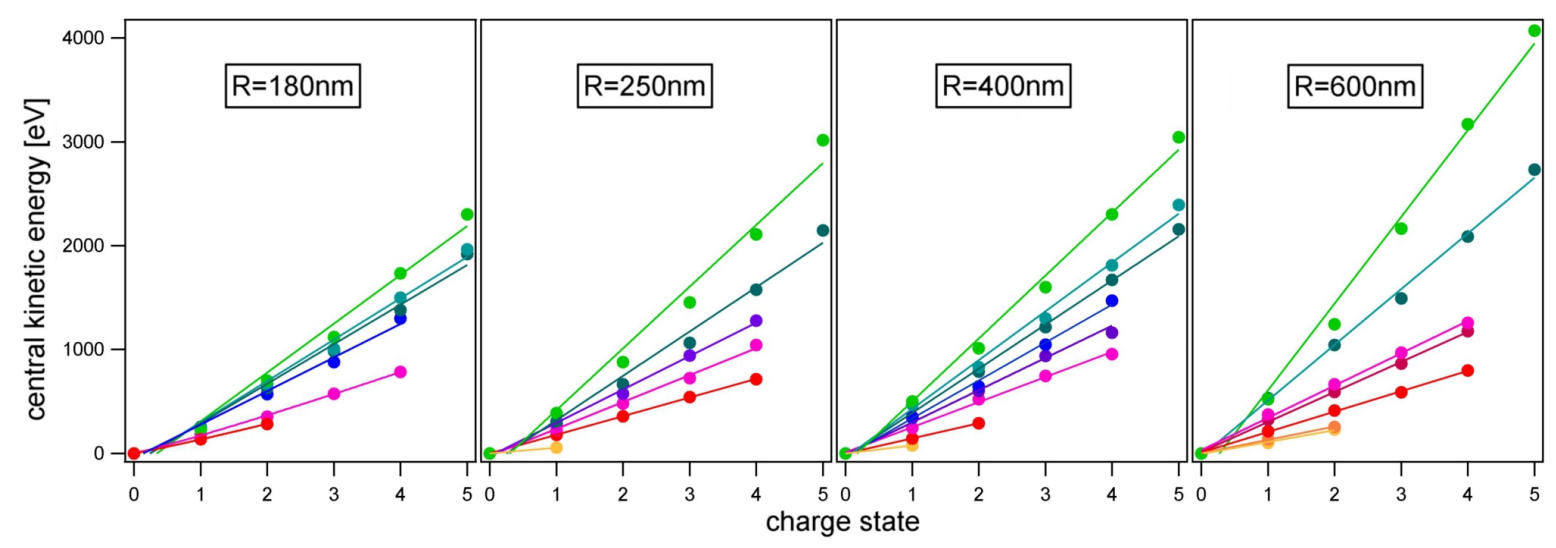

Figure 4.23: Central kinetic energy per charge state. The color coding corresponds to epd values consistent with Fig. 4.21. The kinetic energies derived from the scans in Fig. 4.21 for $R=180-$ $600 \mathrm{~nm}$ are mainly linear functions of the charge states, although the highest epd spectra reveal small deviations towards a more quadratic correlation. Almost identical curves arise for spectra from different cluster sizes but same epd value.

For large clusters from 180 to $600 \mathrm{~nm}$ radius a reliable assignment of the features in the ion spectra is possible and clear maxima of each charge state can be found. Therefore, the central $E_{k i n}(q)$ can be determined by the time-to-energy conversion established in the SIMION simulation. The results are presented in Fig. 4.23.

The colors of the data points correspond to the color coding in Fig. 4.21 and can thereby be assigned to epd-values. Mostly linear dependencies arise for all spectra and only the most intense hits in Fig. 4.23 from clusters with $R=400 \mathrm{~nm}$ and $R=600 \mathrm{~nm}$ reveal a slight deviance towards a more quadratic correlation. For smaller clusters with $R=180 \mathrm{~nm}$ a positive bending is observed already for the blue curve, i.e. at smaller epd values.

The slopes of all curves increase only approximately by a factor of 3 to 4 , while at the same time the exposure power density increases by one to two orders of magnitude (cf. Fig. 4.22). Also the size dependence of the kinetic energies has to be considered very weak. The maximal kinetic energy increases only by a factor of 1.7 (5+ in the light-green curves from $2.3 \mathrm{keV}$ at $180 \mathrm{~nm}$ to $4 \mathrm{keV}$ at $600 \mathrm{~nm}$ ), while the particle radius increases by a factor of 3.3. For other than the green curves in Fig. 4.23, the slopes of $E(q)$ for similar epd values (same colors) even remain almost constant with increasing cluster size. These observations are of importance in the considerations of the mechanism, which leads to the acceleration of the ions in section 4.4.5.

Up to this point from the analysis of size and epd sorted ion spectra, the following trends can be summarized: For very large clusters with radius of $250 \mathrm{~nm}$ or bigger, an increase of the abundance of higher charge states with rising epd value is observed, accompanied by shifts of the peaks towards higher initial kinetic energy. The charge states appear in rather narrow distributions around a central kinetic energy. The central kinetic energies are mainly linear functions of the charge states. A slow part of the distribution towards zero kinetic energy only appears for the $\mathrm{Xe}^{1+}$ feature and is absent for higher charge states. While some of those tendencies are also found for smaller cluster sizes $(R=80 \mathrm{~nm}, 180 \mathrm{~nm})$, changes in the energy distributions of the charge states (i.e. the shape of the peaks) and small deviations from the purely linear behavior towards a more quadratic dependency of the central energies are observed. This observation might tie in with earlier results on ensembles of smaller xenon clusters at $90 \mathrm{eV}$, where a quadratic correlation for $E_{k i n}(q)$ was found [137, 94]. 


\subsubsection{Recombination versus expansion in a large dense nanoplasma}

The findings described above are compared to previous results in this section for a detailed discussion of the underlying mechanisms and dynamics. Concepts which were introduced in section 2.3 are used to enable a further interpretation of the increasing kinetic energies with exposure power density. As discussed in detail below, distinct differences in the present study from earlier results indicate, that the measured ions are virtually all ejected from the surface of a quasi-neutral cluster plasma, which efficiently recombines and disintegrates into mostly neutral particles.

The nanoplasma dynamics of xenon clusters at $90 \mathrm{eV}$ photon energy have been investigated in previous experimental $[80,32,94,31]$ and theoretical $[79,59,75]$ studies. Because of the high cross-section of xenon at $90 \mathrm{eV}$ photon energy due to the resonant $4 d-\epsilon f$ transitions, the atoms of the cluster become multiply charged (cf. section 2.2.2, Fig. 2.12 [51]). In the beginning of the pulse, the electrons are released from the atoms with a kinetic energy of about 20 to $30 \mathrm{eV}$ (cf. section 2.3.3, Fig. 2.18 and [80]) and soon get trapped in the rising cluster potential.

The number of electrons $n_{e, \text { out }}$, which can leave the cluster by direct photo ionization before they are trapped in the developing Coulomb potential of the cluster can be estimated from the simple model of a charged sphere (see section 2.3.2, Eq. 2.77) to be

$$
n_{e, \text { out }}=\left(h \nu-I_{p}\right) \frac{4 \pi \cdot \epsilon_{0} \cdot R}{e^{2}}
$$

For a xenon cluster with $400 \mathrm{~nm}$ radius, irradiated with $90 \mathrm{eV}$ photons, the maximal kinetic energy is defined by the Auger electrons with $\approx 32 \mathrm{eV}[80,79]$, not by the difference between photon energy and ionization potential $h \nu-I_{p}=22 \mathrm{eV}$.

Only about 9000 electrons can escape the Coulomb potential of the cluster compound consisting of $5 \cdot 10^{9}$ atoms. In [80] the electron distributions from $90 \mathrm{eV}$ irradiated xenon clusters were measured, which exhibited a strong component of high kinetic electrons. In the super dense plasma produced in the highly absorbing cluster, efficient thermalization processes lead to a tail in the kinetic energy distribution of electrons with sufficient energy to overcome the Coulomb barrier. Also these electrons will make a contribution to the net charge of the cluster which is not considered in the calculation above.

A basic classification of the regime in which the interaction takes place, proposed in the study of Arbeiter and Fennel [79], can be done by simply using $n_{e, \text { out }}$ from equation 2.77 for estimating the frustration parameter (cf. section 2.3.2), given by the ratio of activated to outer-ionized electrons. The number of activated electrons, i.e. those separated from the parent ion but potentially bound to the cluster as a whole, have to be estimated. They can not be simply calculated from the atomic cross-section and the epd values, as the radius of $400 \mathrm{~nm}$ is considerably larger than the penetration depth in the case of xenon at $90 \mathrm{eV}$ photon energy.

At a maximal epd value of $5 \cdot 10^{14} \mathrm{~W} / \mathrm{cm}^{2}$ the number of photons $n_{\gamma}$ which falls into the geometric cross-section of the cluster can be calculated to $n_{\gamma}=1.7 \cdot 10^{10}$. This exceeds the number of atoms in the cluster of $5 \cdot 10^{9}$, therefore the simple approximation is used that an average charge per atom of 1 is produced in the cluster. The frustration parameter can be calculated to

$$
\alpha=\frac{n_{\text {photo }}}{n_{e, \text { out }}}=\frac{5 \cdot 10^{9}}{9000}=6 \cdot 10^{5} .
$$

On the other hand, the lowest identifiable power density (cf. section 4.4.3) of $3 \cdot 10^{12} \frac{\mathrm{W}}{\mathrm{cm}^{2}}$ leads 

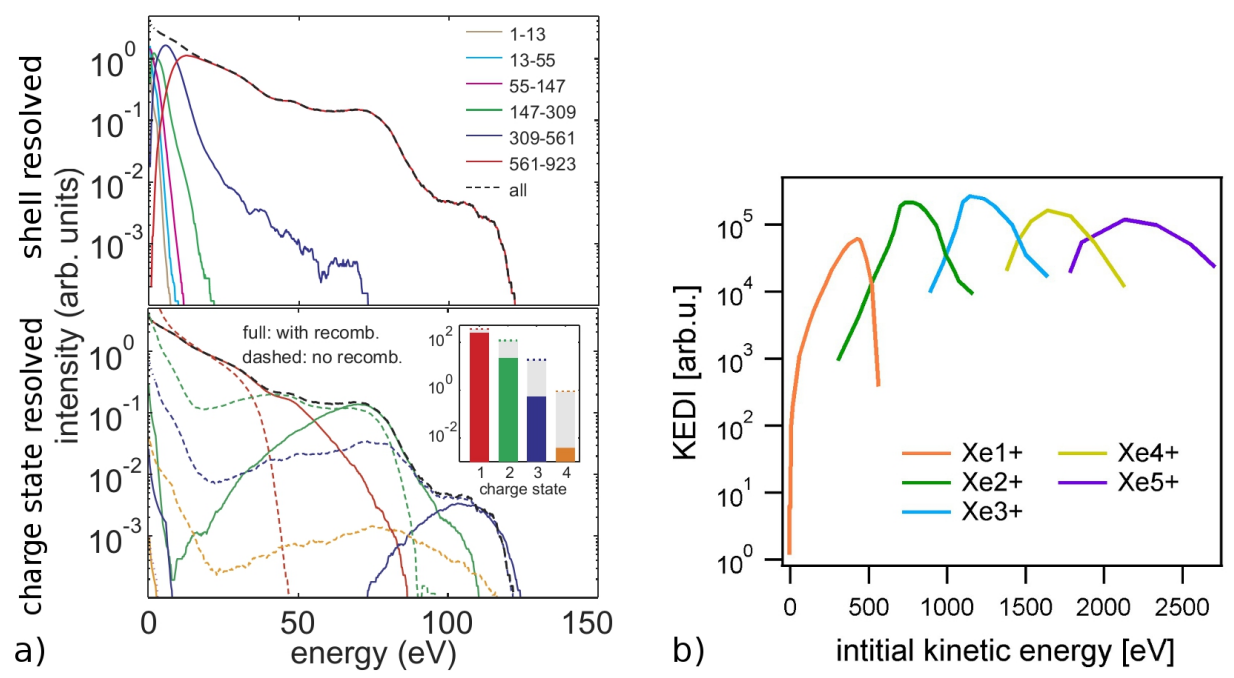

Figure 4.24: a) Kinetic energy distribution of ions resolved for cluster shell (upper panel) and charge state (lower panel) was calculated for the hydrodynamic expansion of an argon cluster with $N=1000$ atoms. The difference between dashed and solid lines in the lower panel emphasizes the role of recombination effects for the measured ion spectra [79]. b) Experimentally obtained KEDI a xenon cluster with $\mathrm{R}=400 \mathrm{~nm}$ at an epd of $1.8 \cdot 10^{14} \mathrm{~W} / \mathrm{cm}^{2}$ (cf. section 4.4.2).

to $1 \cdot 10^{8}$ photons in the cluster cross-section, and thus to a lower limit for the $400 \mathrm{~nm}$ scan of $\alpha=1 \cdot 10^{4}$. Therefore, the studied large clusters are considered to reside deeply in the nanoplasma regime. The occurrence of a highly dense plasma is accompanied by a strong recombination of the screened cluster core [31].

The dominating expansion mechanism in the large systems according to the $\alpha$-values of $>10^{4}$ is expected to be due to hydrodynamic forces. In contrast, earlier results on ensembles of smaller clusters $[94,137]$ with $\alpha$-values of $40-1400$ were interpreted as signatures from a combination of a Coulomb-exploding shell with a thickness of 20 to 30 of the cluster radius and a hydrodynamically expanding core. There, the central kinetic energy deduced from modeling the ion spectra was found to be quadratic [94]. As the analysis of in the last section also indicates a transition from a purely linear to a more quadratic behavior towards smaller systems, both findings might be linked by the cluster size.

Also in studies on the explosion dynamics of rare-gas clusters in strong infrared laser pulses [90] effects of both expansion mechanisms were found by investigating the behavior of $E_{k i n}(q)$. For rather large xenon clusters $\left(<N>=2 \cdot 10^{6}\right)$ a quadratic increase of the kinetic energy for lower charge states was replaced by a linear dependency for higher charge states. In contrast to the interpretation in [90], simulations in [95] explained this behavior as an effect of the focal profile of laser power density in a purely Coulomb-explosion picture. At least in the current results effects of the focal distribution can be excluded as the measurements have been carried out on single clusters under consideration of the epd.

The ambiguity of interpreting kinetic energy distributions of ions is also demonstrated in the study of Arbeiter and Fennel [79], where ionization, recombination and expansion dynamics from high to low $\alpha$-values are traced. Some results of this study have already been discussed in section 2.3.3. In particular when recombination effects are considered, very similar oscillatory patterns in the kinetic energy distributions of ions are obtained for scenarios undergoing a Coulomb-explosion as well as for hydrodynamic expansion. Fig. 4.24 a displays a shell and a charge state resolved analysis of the simulation of a laser-cluster interaction with large 
$\alpha$-values, i.e. hydrodynamic expansion. For comparison in Fig. 4.24b the experimentally obtained KEDI is displayed, which has already been presented above in Fig. 4.20 c. The cluster size investigated in the theoretical study was much smaller than in the current experiment $(N=1000)$. Nevertheless some features occur in the simulated distribution in Fig. 4.24 a, which are very interesting for the interpretation of the current ion spectra:

- Ions with high kinetic energy only emerge from the outermost shells (upper panel).

- High kinetic energies only arise for high charge states (lower panel).

- The higher charge states also lack of a low kinetic part due to recombination (lower panel, solid compared to dashed curve).

- The central kinetic energy per charge state appears to depend linearly on the charge state (lower panel, $E(2+) \approx 70 \mathrm{eV}, E(3+) \approx 105 \mathrm{eV}$ ).

These features are found also in a very pronounced manner in the current results of Fig. 4.24b. But in contrast to the simulated energy distributions, also the $\mathrm{Xe}^{1+}$ peak reveals only a very low contribution towards lower energies and all higher charge states appear to be depleted at the low energy side due to recombination. In particular the absence of low and zero kinetic energies in the case of $\mathrm{Xe}^{1+}$ has to be interpreted as a signature of strong recombination effects in the large clusters down to fully neutral atoms.

The agreement between the experiment and the simulation supports the idea of an ejected ionic surface from a fully recombining compound. The extreme conditions of the experiment, i.e. the large size of the particles and the massively dominant recombination in the almost neutral dense plasma enable a satisfying description based on simple electrostatic models. They are described and discussed in the following section.

\subsubsection{Net charge versus charge separation at the surface}

The narrow energy distributions and the absence of low kinetic ions for all charge states down to $\mathrm{Xe}^{1+}$ indicates complete recombination of a large part of the cluster, as discussed in the last section. In order to quantify the thickness of the exploding surface and specify the underlying mechanism, an electrostatic model will be discussed in this section of a fully screened cluster plasma with deviations from neutral occurring only at the surface. The model is used to calculate an effective charge on the clusters from the kinetic energies of Fig. 4.23.

Model of a charged surface: The central kinetic energy of the energy distributions, as derived above from the ion spectra of single large clusters, was found to be a linear function of $q$. A purely linear dependency on $q$ would also arise from the simple electrostatic picture of a shell of charged ions on the surface of an otherwise neutral cluster plasma. The radial distributions of plasma electrons and ions are sketched in Fig. 4.25 a. The basic assumption of this static model is the full screening of the inner part of the cluster up to a distinct radius $R^{\prime}$. Unscreened surface ions are then driven by the Coulomb repulsion of each other and explode off. In the case of a large cluster, i.e. $R \gg R-R^{\prime}$, the kinetic energy of the ions will be proportional to $Q \cdot q$, as the kinetic energy of a charge $q$ moving from the surface of 


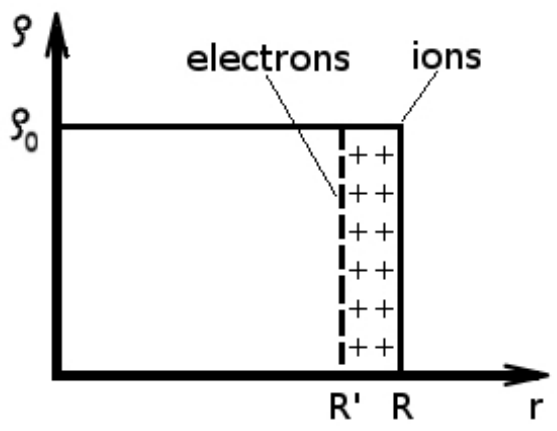

a) Limit $Q=N, T \rightarrow 0$

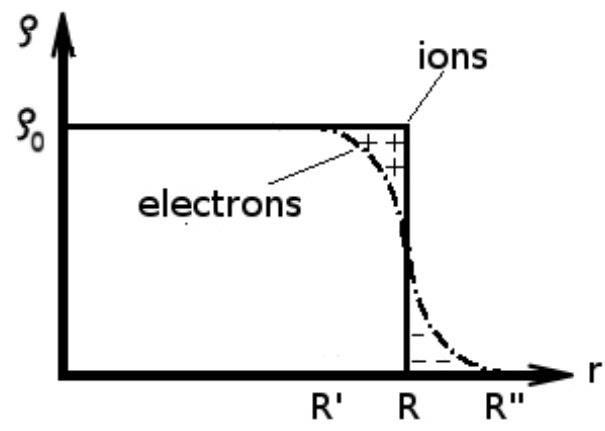

b) $\quad$ Limit $Q=0, T>0$

Figure 4.25: a) Electrostatic picture of a shell of charged ions on the surface of an otherwise neutral cluster plasma. Full screening of the inner part of the cluster up to a distinct radius $R^{\prime}$ is assumed, neglecting the temperature of the electron gas. b) Electrostatic model of the hydrodynamic effect at the surface. The finite electron temperature leads to a further extended electron cloud up to $R^{\prime \prime}$ and therefore to a partially unscreened region in the cluster between $R^{\prime}$ and $R$. Aside from this surface dipole field due to the electron spill-out, the cluster is neutral.

a charged sphere to infinity [157] is given by

$$
E_{k i n}=\frac{1}{4 \pi \epsilon_{0}} \cdot \frac{q \cdot Q}{R} .
$$

The assumption of a full screening of the inner part of the cluster is equivalent to neglecting the temperature of the electron gas. In fact, as discussed in the last section by means of the frustration parameter $\alpha$, the quasi-free electrons will screen the charge from the ions almost completely and form a rather cold nanoplasma. On the other hand, the observation of "hot" electrons [80], i.e. the high energetic tail of electrons from the thermalization process point to the limit of the model of a Coulomb-exploding shell, as the electron temperature clearly can not be neglected.

Model of a surface dipole field: In the close vicinity of the surface, deviations from neutrality occur even in a fully neutral plasma. In particular, the electron cloud will exceed the boundaries of the ion distribution, referred to as electron spill-out [103]. This hydrodynamic effect can be accounted for in a complementary, but also static model, which is displayed in Fig. $4.25 \mathrm{~b}$. The total charge on the cluster is neglected but the finite electron temperature renders its contribution. The ions on the surface of the cluster experience a Coulomb force despite the neutrality of the cluster as a whole, as they encounter a repulsion from only partially screened ions in the surface shells. Within this model the kinetic energy of the ions ejected from the surface would also increase linearly with their charge state $q$, as the hydrodynamic force is in principle also a Coulombic repulsion from a positively charged surface.

During and shortly after the interaction with the laser pulse, a condition somewhere in between the limiting cases of Fig. 4.25 might exist in the cluster, where surface ions are accelerated radially by the combination of the total charge on the cluster and an unscreened surface region induced by the finite electron temperature. Beyond that instant in time, an electrostatic approach must fail to describe the real dynamics of the process, as the ion motion is slow and therefore the evolution of the nanoplasma can influence the ion distribution for a very long time. 


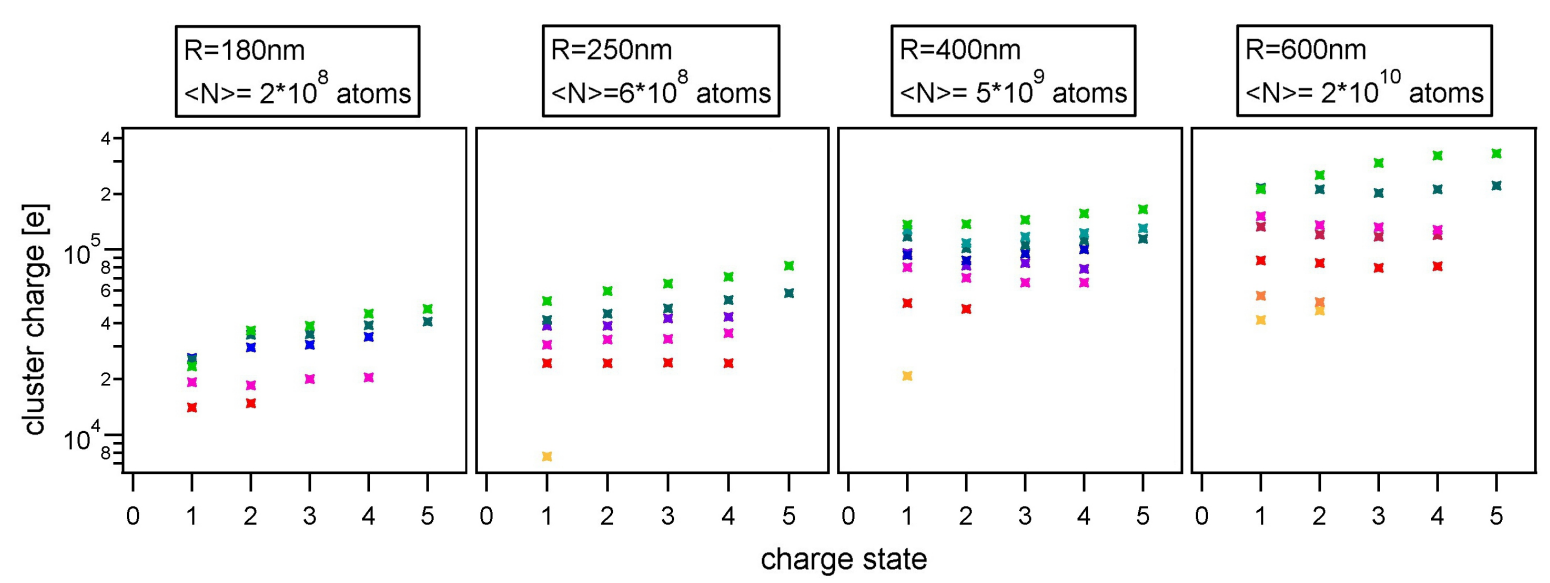

Figure 4.26: Total charge on the clusters from 180 to $600 \mathrm{~nm}$ radius, calculated from the electrostatic model of a charged surface. The color-coding corresponds to increasing epd values from yellow to green, in consistency to the color-coding in the previous figures 4.21 and 4.23. Approximately constant curves are observed for larger clusters and lower and medium epd-values. Positive slopes arise at cluster sizes $\geq 400 \mathrm{~nm}$ only for hits with maximal epd. Towards smaller clusters increasing $Q(q)$ values are also observed for lower epd values.

On the other hand, the use of a static picture can be justified by the idea, that the recombination of the nanoplasma happens very fast compared to the expansion of the cluster. An indication therefore is given by the efficient recombination down to neutral atoms within the cluster. As discussed in section 2.3.2, the probabilities for recombination are only high as long as high densities of ions allow for collisions. Also the fact that the model of a charged sphere agrees well with the measured distributions gives a hint that the cluster contributes to the time-of-flight spectra mainly in the very beginning of the expansion. In a later phase the highly charged ions which were screened by the cooler part of the electron distribution have efficiently recombined with the cold and dense electron cloud down to neutral atoms and disappear from the time-of-flight signal.

Effective cluster charge: In order to quantify the contributions from total cluster charge and electron spill-out, an effective total charge on the clusters $Q_{\text {total }}$ is derived from the central kinetic energies by using the model in Fig. 4.25 a. Eq. 4.2 is resolved for $Q$, to calculate an effective total charge separately for each charge state in the spectra

$$
Q_{\text {total }}=0.69 \cdot E_{\text {kin }}[\text { in } \mathrm{eV}] \cdot R[\text { in } \mathrm{nm}] \cdot \mathrm{q}^{-1} .
$$

The effective charges calculated from the size sorted epd scans with $R=180$ to $600 \mathrm{~nm}$ are displayed in Fig. 4.26. The color-coding in Fig. 4.26 from yellow to green corresponds to increasing epd values and is consistent with the colors used in the scans in Fig. 4.21 and Fig. 4.23.

If the kinetic energies presented above in Fig. 4.23 would be purely linear functions of the charge states, a constant value of $Q_{\text {total }}$ would be expected for every charge state of a distinct hit. A closer examination of the curves in Fig. 4.26 reveals a rather constant value for many spectra. But in accordance to the deviations from linearity of the central kinetic energy per charge state, as discussed in section 4.4.3, also a positive slope can be observed in some cases. Towards smaller cluster size, the calculated total charge exhibits a constant value for $Q(q)$ only for lowest epd values. On the other hand, for larger clusters only the most intense hits deviate from constant and reveal an increasing value of the effective total charge with charge state. Note that slight deviations for $\mathrm{Xe}^{1+}$ in the $R=400$ and $600 \mathrm{~nm}$ scans can be 


\begin{tabular}{|l|l|l|l|l|}
\hline radius & $180 \mathrm{~nm}$ & $250 \mathrm{~nm}$ & $400 \mathrm{~nm}$ & $600 \mathrm{~nm}$ \\
\hline$Q_{\text {total }}$ & $4.8 \cdot 10^{4}$ & $8.5 \cdot 10^{4}$ & $1.7 \cdot 10^{5}$ & $3.5 \cdot 10^{5}$ \\
\hline$N_{\text {surface }}$ & $5.6 \cdot 10^{5}$ & $1.1 \cdot 10^{6}$ & $2.8 \cdot 10^{6}$ & $6.3 \cdot 10^{6}$ \\
\hline$n_{e, \text { out }}$ & $4.0 \cdot 10^{3}$ & $5.6 \cdot 10^{3}$ & $8.9 \cdot 10^{3}$ & $1.3 \cdot 10^{4}$ \\
\hline
\end{tabular}

Table 4.2: Comparison of effective charge, number of atoms in the outermost atomic shell and number of outer ionized electrons.

identified as uncertainties for extracting the central kinetic energy due to the close vicinity of the enhanced $\mathrm{Xe}^{2+}$ back-peak in the spectra.

The behavior towards smaller clusters might be interpreted as the onset of Coulomb explosion of a more extended outer shell, as discussed in [94, 137]. But also the purely hydrodynamic picture of an exploding surface shell of ions can offer an explanation for the increasing effective charge with charge state. A constant value for the effective total charge occurs when all ions experience the repulsion from the whole surface charge. But as they are accelerated proportionally to their charge, soon the expansion of the surface ions will evolve in layers of different charge states, which expand with different velocities. An illustration for the idea of expanding layers can be found in Fig.4.27. For hits which have experienced high epd, the total number of highly charged ions constitutes a considerable fraction of all ions. Thus, in the evolving expansion, the ions will only experience charges from their own "expansion layer" and the ones of lower charged ions, while charges from faster expanding layers cancel themselves out.

The number of effective charges on the clusters calculated by Eq. 4.3 for all analyzed hits from $R=180$ to $600 \mathrm{~nm}$ reaches from about $1 \cdot 10^{4}$ up to $3 \cdot 10^{5}$. The effective charges can be compared to other characteristic values of the cluster, which are displayed in table 4.2. Even for the maximal reached values, $Q_{\text {total,max }}$ only corresponds to a small fraction of the number of atoms $N_{\text {surface }}$ in the first atomic layer. On the other hand, the values of $Q_{\text {total }}$ are 10 to 30 times higher than the number of electrons $n_{e, \text { out }}$, which can be directly outer ionized before frustration sets in (cf. Eq. 2.77). Even if the effect of thermalization is included by assuming a much higher value of the electron kinetic energy of $100 \mathrm{eV}, Q_{\text {total }}$ remains considerably larger than $n_{e, \text { out }}$. This clearly indicates, that the main mechanism transferring energy into the surface ions is not the cluster charge but the electron spill-out. Therefore $Q_{\text {total }}$ can not be considered as a good measure for the absolute number of ions ejected from the surface before complete recombination.

It would be helpful for these considerations to estimate the total number of ions leaving the cluster directly from the measured spectra. But due to the unknown gain function of the MCP, absolute numbers of particles can not be calculated. Nevertheless, if the gain function is assumed to be constant for all xenon charge states and initial kinetic energies (cf. section 3.2.4 and reference [141]), a rough estimate of a lower limit can be gained from the transmission corrected KEDI distribution of one ion spectrum, which has been presented before in figure 4.20c and compared to simulations in Fig. 4.24.

For this estimate of a minimal number of ions which have been ejected from the clusters, the KEDI spectrum is scaled in such a way that the detected signal of $\mathrm{Xe}^{1+}$ with zero kinetic energy (transmission through the spectrometer $=1$ ) corresponds to one single ion. The integrated area under the peaks then amounts to a number of $3.5 \cdot 10^{6}$ ions. This about a factor of 1.3 more than the number of atoms in the outermost atomic layer of the cluster. Therefore also this rough estimate supports the idea of a thin surface exploding of the cluster. 


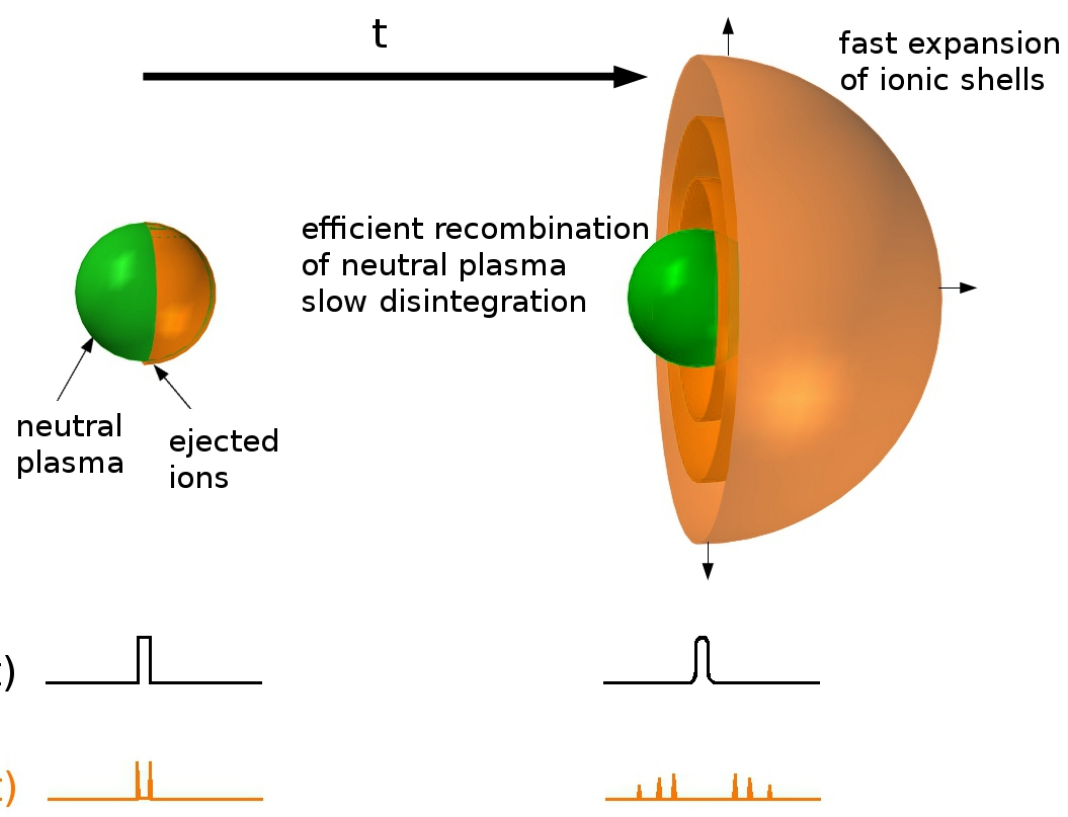

Figure 4.27: Illustration of surface explosion and core recombination.

Another approach to estimate the thickness of the outer shell participating to the ion spectrum could be made by investigating the width of $\approx 200 \mathrm{eV}$ of the kinetic energy distributions around a center value. In particular, this could give insight in the temporal evolution of the electron temperature and the resulting spill-out of the electron cloud. However, this point in the discussion clearly marks the limit of a static analysis of the circumstances and more sophisticated modeling will be required.

Current discussions with the group of Thomas Fennel indicate, that it will be possible to simulate the temperature of the quasi-free electron in order to explain the measured kinetic energies. This would be a possibility to test the contributions of ionization heating and other processes influencing the electron temperature. Preliminary estimates indicate only weak changes in the potential induced by the electron spill-out for changing density of the quasi-free electrons. Also the cluster size appears to play only a minor role. This would be in good agreement to the observations of weak changes in the ion kinetic energies with size and exposure power density in section 4.4.3.

As a summarizing conclusion of the analysis, the initial kinetic energy distributions indicate that for large clusters all detected ions come from the outermost surface of the clusters. The absolute values of the kinetic energy reveal that the main mechanism of driving the ions is the spill-out of the electron cloud, not the total charge on the cluster. While the atoms of the cluster are expected to be transiently highly ionized, the dominant fraction of the cluster will recombine to neutral state.

In this context it is interesting to note, that the biggest part of the cluster does not experience Coulombic forces at any point. Regardless of possible transient high charge states the ions remain fully screened until they recombine to neutral atoms which also takes away a driving force for the expansion. Thus, the question arises of what happens to this compound, which is only bound by the weak Van-der-Waals force in the first place and can be expected to have gained much thermal energy due to collision processes. Studying the timescale of the disintegration of the remains of the clusters on long time scales promises to be a fascinating topic for the analysis of time-resolved experiments up to nanosecond delays [158]. 


\subsection{Fingerprints of a nanoplasma shell in size-selected scat- tering profiles}

As discussed in the last section, the ion spectra of single large xenon clusters contain only ions from the outermost surface of the large, dense and quasi-neutral nanoplasma which evolves in the XUV laser-cluster interaction. For the largest part of the cluster, efficient recombination leads to neutral residuals which can not be detected by the ion spectrometer.

In contrast, during the interaction with the FEL pulse the photons will deeply penetrate the cluster surface and interact with all constituents of the nanoplasma. Thus the conditions within the nanoplasma including transiently high charge states and the density of the quasifree electrons will appear encoded in the elastically scattered light. In the scattering patterns of the very same large clusters, which have been analyzed according to their ion spectra in the last section, a modulation is found which indicates the presence of a shell structure within the nanoplasma.

This observation demonstrates that even though changes in the geometry of the cluster on the timescale of the light pulse due to nuclear motion can be excluded, the transient changes of the electronic properties are able to clearly alter the scattering pattern. It can be considered surprising how pronounced the modulations are as the exposure time of $100 \mathrm{fs}$ is much longer than the time scale of electron motion. Analyzing the possibilities for the generation of such a modulation reveals the necessary precondition of a sharp interface within the cluster plasma, which appears difficult to explain.

This chapter is organized as follows: In section 4.5.1, the size- and epd-selected scattering patterns are analyzed for power density dependent developments. The intensity profiles derived from the scattering patterns of clusters with radii from 180 to $600 \mathrm{~nm}$ exhibit a developing superstructure, which is behaving similarly for all cluster sizes. In section 4.5.2 a time-binned approach for analyzing the observed development is introduced. The individual contributions from a sequence of intermediate states can be investigated by examining the differences of angular intensity profiles of the scattering patterns.

In the simplest approximation, the modulation can be understood as the diffraction from a ring instead of a full sphere. More detailed insight can be gained with Mie-based simulations for coated spheres in section 4.5.3. The influence of the parameters of the outer shell, i.e. shell thickness $d$ and refractive index, are studied. Based on the characteristic changes in the data, an attempt is made to match the measured scattering profiles with a simulated sequence of intensity profiles. The fit parameters yield a low absorbing shell with increasing thickness and decreasing real part of the refractive index. An abrupt change in the real part is found to be necessary for producing the beating pattern.

In section 4.5.4 the special absorption characteristics of xenon atoms and ions around $90 \mathrm{eV}$ photon, which have been presented in chapter 2.2.2, are summarized and set into the context of the plasma environment. By modeling the ionization dynamics of the cluster in a simple one-dimensional approach in section 4.5.5, the radial development of charge state distributions and the density of the quasi-free electrons are investigated. The radially changing local electric field, acting on the energy levels of the ions together with the resonant behavior of xenon in the vicinity of the excitation energy, might be able to explain the presence of a highly reflecting layer tens of nanometers within the plasma. 

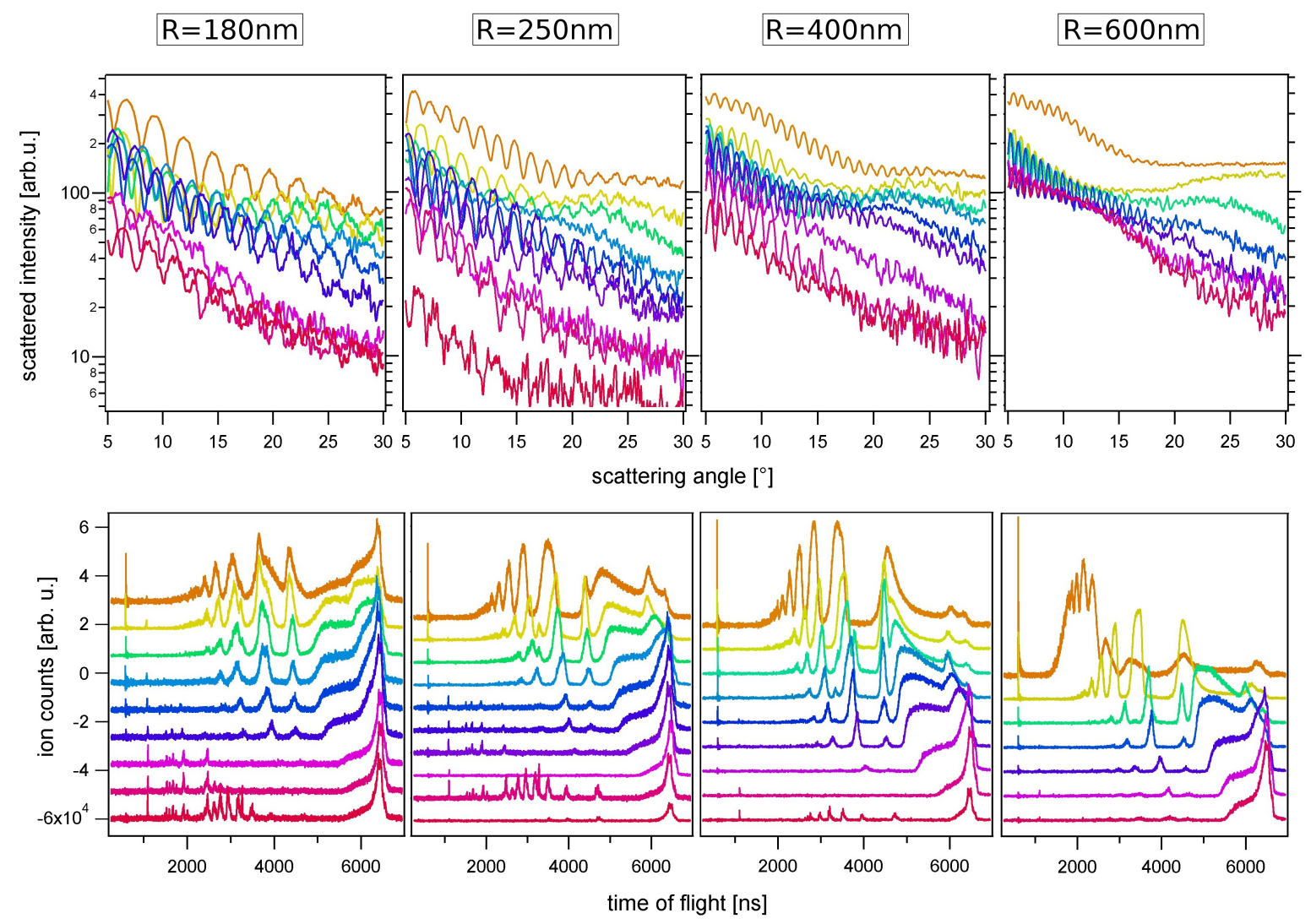

Figure 4.28: Size-sorted epd scans for radii 180 - $600 \mathrm{~nm}$. In the first row, the radial profiles of the scattering patterns are plotted over scattering angle. Low frequency modulations superimpose the high frequency oscillations, which reflect the cluster size. They reveal a clear power density dependence but only a weak cluster size dependence. The corresponding ion spectra in the second row show the concurrent characteristic changes in the particle disintegration (cf. section 4.4).

\subsubsection{Modulation in size-sorted scattering profiles}

In Fig. 4.28 the same data sets are displayed that have been analyzed in the previous chapter for power density dependent effects in the ion spectra. In this chapter, the development with increasing exposure power density in the scattering patterns is discussed. In the first row of Fig. 4.28, the radially averaged intensity profiles of the CCD images are plotted versus scattering angle $\theta$.

To obtain the intensity profiles, the intensity of each image pixel was corrected for the flat detector by multiplying with $\cos (\theta)^{-3}$ [32]. This factor consists of a term for the quadratically decreasing intensity with increasing distance to the interaction region, which is taken into account by a factor of $\cos (\theta)^{-2}$, and the smaller effective pixel area, which can be corrected by multiplying with another factor of $\cos (\theta)^{-1}$. For radially averaging, the CCD image was restricted to a segment on the right side of the detector, where no ion signal, low stray light and no deviations from a flat efficiency were observed. Detector areas with a decreased efficiency and the linear response of the scattering detector are investigated in more detail in the appendix of this thesis. As a conclusion from these investigations a deviation from a linear response has to be considered at least for the highest signal intensities which are found at small scattering angles. Thus, only structures and developments in the patterns, not absolute intensity values will be discussed in the further analysis. 

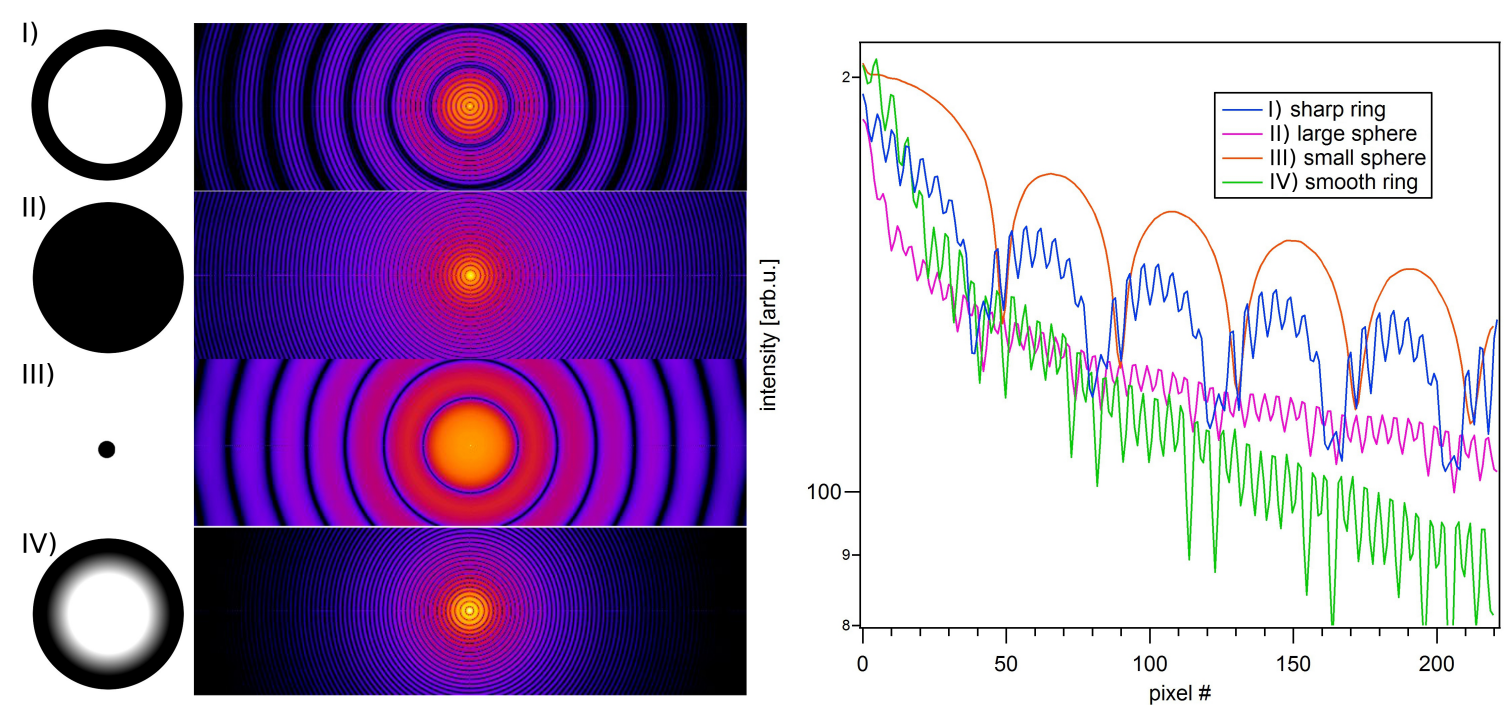

Figure 4.29: a) 2D Fourier transformation of the outline of a ring reveals a modulation in the radial profile b) Comparison with a full sphere indicates, that the fine oscillations reflect the size of the whole object. c) Comparison with a small sphere of the same diameter as the ring thickness shows, that the frequency of the superposing modulation corresponds to the thickness of the ring. d) Sharp interfaces are necessary to yield a modulation. If one interface is washed out, the beating pattern vanishes.

The scattering profiles displayed in Fig. 4.28 show the fine lobes of the diffraction rings, which become narrower for larger clusters. In addition, the more intense hits of every cluster size reveal a low-frequency oscillatory pattern, which superimposes the maxima and minima of the diffraction rings. The broad, wave-like superstructure becomes more prominent for increasing epd and appears to shift to larger scattering angles.

As discussed in section 2.1.3, characteristic structures in the scattering patterns correspond to characteristic length scales within the scatterers. One characteristic length scale is obviously given by the overall size of the particle. This results in the high-frequency oscillatory structures of the profiles becoming finer with increasing cluster size. The low frequency modulations indicate a second characteristic length scale in the particles. The superstructure appears to be very similar from 180 up to $600 \mathrm{~nm}$ radius with a global minimum observed roughly at $\theta=15^{\circ}$. This allows already for a very rough estimate of the corresponding characteristic length scale of about $30 \mathrm{~nm}$, calculated from the first minimum of an Airy pattern.

In Fig. 4.29 a two-dimensional illustration of the effect is presented. The 2D Fourier transform of the black and white outline of a ring (I) exhibits a pronounced superstructure in addition to the fine ring structure. A comparison with the patterns of a full sphere instead of the ring (outline II) confirms that the frequency of the fine ring structure corresponds to the overall size of the scatterer. On the other hand, the slowly changing superstructure represents the thickness of the ring. This is indicated by the pattern from a small sphere (outline III) with the diameter of the thickness of the ring, which reveals maxima and minima similar to the modulation from the first pattern. The contributions to the beating pattern can be followed even more clearly in the radial intensity profiles, given in the right panel.

These simple considerations can be applied to the case of the measured scattering patterns of large xenon clusters. The modulations can be understood as a development of an outer shell with different scattering properties compared to the core. The parameters of the shell, such 
as thickness and optical constants, appear to correlate strongly with epd but only weakly with cluster size.

Nuclear motion as a cause for the observed changes can be excluded as discussed in the previous chapter. In the whole expansion process, only a very thin surface in the range of monolayers is expelled from the cluster due to Coulombic forces. The rest of the cluster is expected to expand on a much longer time scale. In addition, even the fastest surface ions start with zero velocity. An estimate can be calculated from the effective charge on the clusters discussed in the previous section (cf. Fig. 4.26). By calculating the forces and the acceleration for the ions with maximal detected kinetic energy $\left(\mathrm{Xe}^{5+}, R=600 \mathrm{~nm}\right.$, $E_{k i n}=4 \mathrm{keV}$ ), a distance passed within $100 \mathrm{fs}$ of less than one Angstrom is found. Even if the final velocity $\left(v=7 \cdot 10^{4} \mathrm{~m} / \mathrm{s}\right)$ was assumed for the ion motion, the ions would only travel $8 \mathrm{~nm}$ within the duration of the pulse. In conclusion, no visible changes in the cluster density will occur during the interaction with the FEL pulse.

An important feature for the interpretation of the modulations in the experimentally obtained profiles can be found in the calculated pattern from outline (IV) in Fig. 4.29. Here, one of the sharp interfaces of the ring structure is washed out, which leads to a virtually complete loss of the beating pattern. Thus, a discontinuity in the optical properties of the nanoplasma at a certain depth within the cluster has to be identified in the subsequent discussions to explain the observed modulations.

For the analysis of the emergence and development of a highly reflecting nanoplasma shell, the modulation effect is studied in detail for the cluster size which is most prominent in the experimental data, $R=400 \mathrm{~nm}$. A time-binning, quasi-static approach for getting insight into the dynamics of the plasma properties is presented in the next section.

\subsubsection{Time-binning of the nanoplasma development}

Imaging a cluster of a well defined size with a distinct exposure power density can not be considered a snapshot of the initial cluster or a single excited state. A more appropriate analogy would be taking a photo of a moving object with an exposure time equalling or exceeding the time scale of the motion. In fact, the scattering pattern consists of the integrated scattering signal from all transient states, which the respective cluster has passed during the interaction.

From these considerations a sequential-static approximation for the analysis of the nanoplasma development is used. This model is based on the assumption of linear processes in the nanoplasma, i.e. processes which are mainly dependent on the number of incident photons and not on the number of photons per time.

Under this assumption the scattering patterns taken from equally sized clusters at different exposure power density can be thought of - again in the analogy of photography - as pictures of the same process, but with different exposure times. In particular, the scattering pattern of the cluster exposed to the minimal photon flux displays the undisturbed cluster and the beginning of the nanoplasma formation. A more intense pattern also contains this information and even more subsequent stages of the nanoplasma development.

The continuous dynamics of the nanoplasma will be approximated by a sequence of static intermediate stages of the development, referred to as time-binning. Sketches of a sequence of such intermediate states through which the plasma evolves during the interaction are displayed in Fig. 4.30. Different exposure power densities will induce and probe the same 

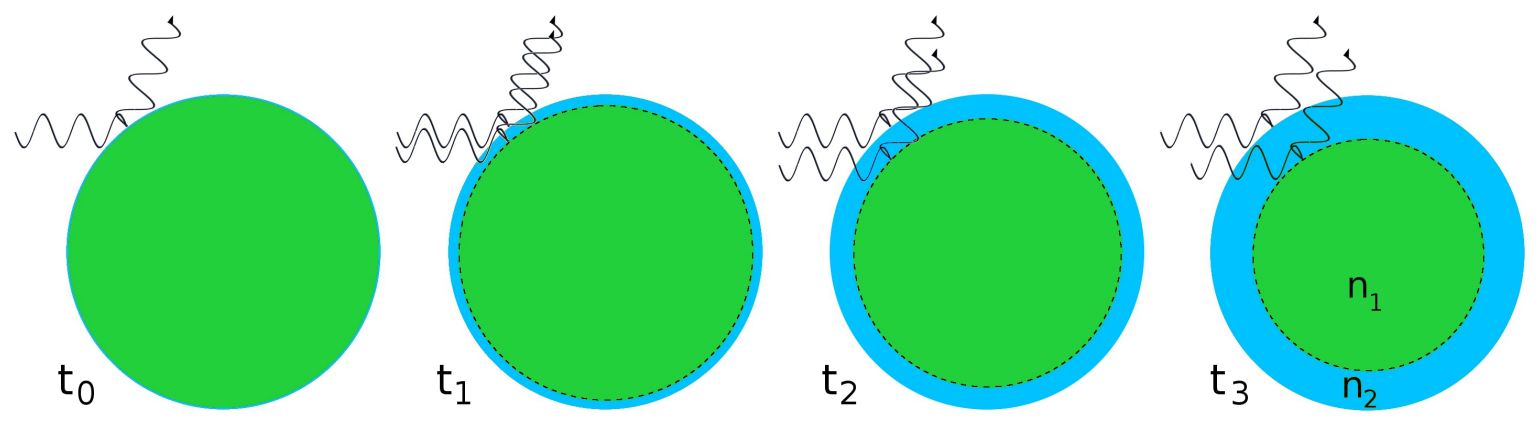

Figure 4.30: Concept of time-binning: During the interaction with the laser pulse a core-shell structure in the nanoplasma evolves. The scattering pattern obtained from the cluster within the duration of the light pulse contains the integrated information over all intermediate states. It can be approximated by the sum of scattering patterns of static intermediate states with average properties of a distinct time bin of the interaction. By analyzing the scattered light from these intermediate states with the static approach of Mie theory, insight into the changing optical properties can be gained.

evolution in the cluster, i.e. the same sequence of intermediate states. But a profile with higher epd contains information on more of these intermediate states. In order to gain snapshots of those intermediate states from the integrated signal of the scattering patterns, the differences between profiles taken at different epd-values are analyzed. This difference signal constitutes the contribution from stages of the development, which were only observed in the more intense pattern, but not quite by the weaker one.

In order to exclude statistical fluctuations in single patterns resulting from shape irregularities and slightly different sizes of the single clusters, only averaged patterns obtained from the cluster size with highest abundance in the data, $R=400 \mathrm{~nm}$ are considered.

In Fig. 4.31 a, the ion spectra of 94 hits of clusters with radii of $400 \pm 50 \mathrm{~nm}$ are sorted by increasing epd values which are derived from the intensity of the light peak at $t=0$ [104]. A manual correction of the vertical position of each spectrum of up to \pm 4 positions was done for aesthetic reasons, resulting in a more homogenous development of the kinetic energies of the ion peaks. Six segments of the epd scan with similar features were merged into averaged ion spectra, which are color-coded respectively. The averaged ion spectra are presented in Fig. 4.31 b.

Absolute epd values are assigned to the averaged spectra in Fig. 4.31 b. Each single spectrum of Fig. 4.31 a was analyzed as follows. The light peak signals were determined by identifying and subtracting the offsets of the spectra, building absolute values, smoothing the absolute spectra and integrating the area under the light peaks. An average light peak signal was calculated for each scan segment. The maximal focal power density of $=5 \cdot 10^{14} \frac{\mathrm{W}}{\mathrm{cm}^{2}}$ (cf. section 3.2.4) was assigned to the uppermost single spectrum. All exposure power densities of the averaged spectra were determined in respect to this one, resulting in values ranging from $3 \cdot 10^{12}$ up to $3 \cdot 10^{14} \mathrm{~W} / \mathrm{cm}^{2}$.

As discussed before, the absolute epd values have to be treated with caution. Even though statistical fluctuations can be considered small in this case, as the signal from the light peaks is averaged over many spectra, the uncertainty remains for the brightest hit which is used for relative assignment of all other values. Further, changes in the scattering signal due to the nanoplasma development and the amount of fluorescence light can not be taken into account. Therefore, also the epd assignment presented in Fig. $4.31 \mathrm{~b}$ should only be used as a guide.

The scattering patterns corresponding to the ion spectra were averaged in the same way. The averaged radial profiles are shown in Fig. $4.31 \mathrm{c}$. The high frequency lobes of the rings in the 

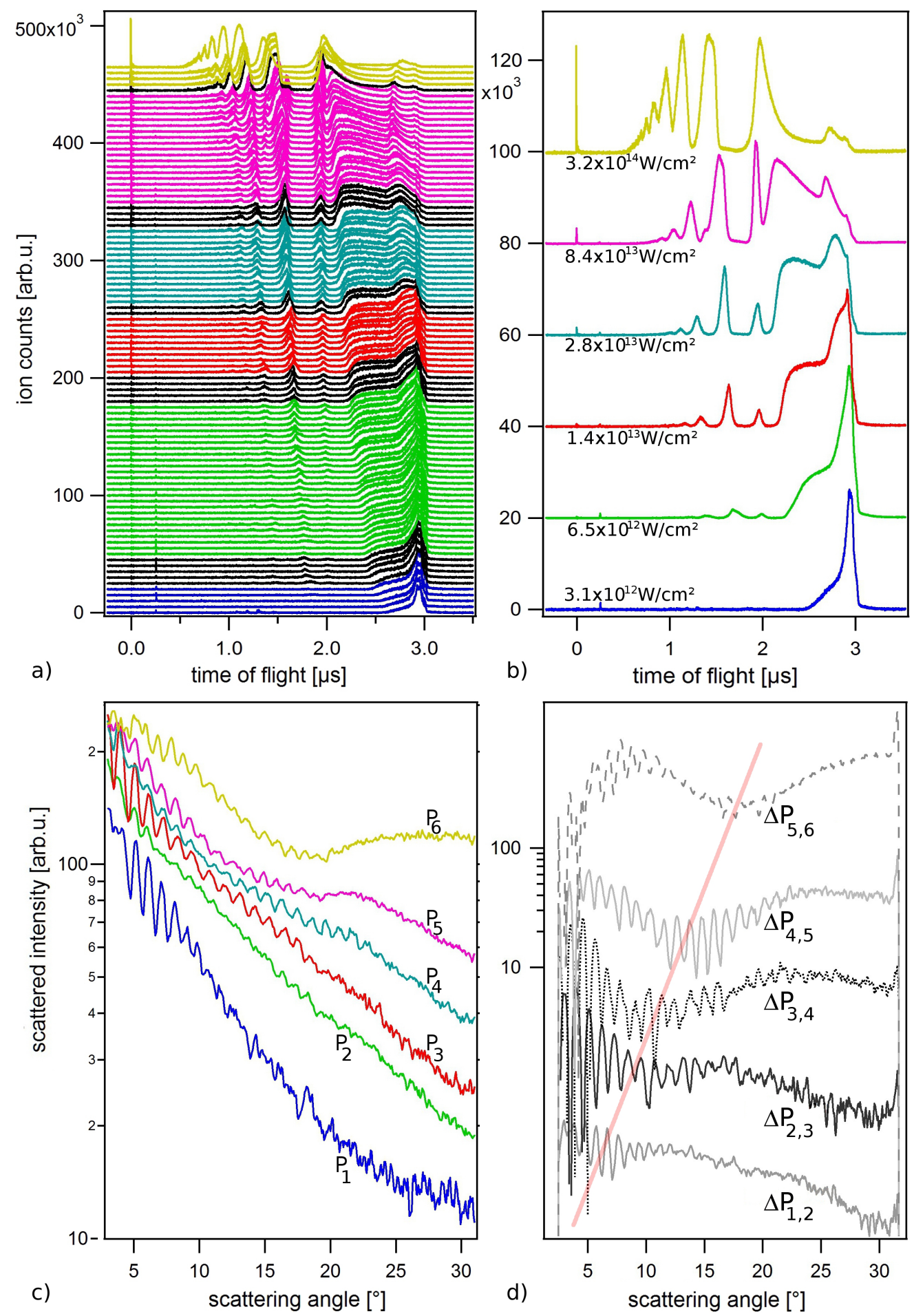

Figure 4.31: Time-binning of the nanoplasma evolution in averaged intensity profiles of $R=400 \mathrm{~nm}$ clusters. a) Ion spectra of the largest available data set of 94 hits from clusters with radii of $400 \pm 50 \mathrm{~nm}$ are sorted by increasing epd from bottom to top. b) Respective to the color code in the full scan, the ion spectra are averaged and assigned to epd values from $3 \cdot 10^{12}$ to $3 \cdot 10^{14} \mathrm{~W} / \mathrm{cm}^{2}$ (see text for details). c) The modulation, which has been observed also in single shot is preserved in the averaged profiles. d) If mainly linear processes of light-matter interaction are assumed, time resolved information can be gained from profiles obtained with different epd values. The contributions from each intermediate state can be extracted by taking the difference between consecutive averaged profiles. The difference profiles reveal a broad lobe with a decreasing width from earlier to later states. At the same time, the minimum of the modulation shifts to larger scattering angles, as suggested by the red line. 
scattering pattern are slightly washed out due to deviations in the sizes of the single clusters. However, the emergence and characteristic development of the modulation with rising epd can be observed clearly in the averaged profiles as an increasing shoulder, which shifts from about 15 to $25^{\circ}$ scattering angle. The averaged profiles are labeled $P_{1}$ to $P_{6}$.

The differences in scattering signal between each two averaged profiles $P_{i+1}-P_{i}$ are plotted in Fig. 4.31 d. They represent the contribution from each interstage $\Delta P_{i, i+1}$. Note that these interstages also constitute the integration over a certain phase of the development.

However, this quasi-static time-binning approach appears to be supported by the gradually developing structures in the difference profiles of Fig. 4.31 d. A lobe-shaped superstructure arises and becomes more prominent from bottom to top, i.e. from earlier to later interstages. Further, the width of the lobe appears to decrease and the visible minimum of the superstructure shifts to higher angles from $\theta \approx 5$ to $17^{\circ}$, as indicated by the red line.

This characteristic behavior can be translated into changing parameters of the refractive core-shell system, which develops in the cluster plasma. Therefore, the curves of Fig. $4.31 \mathrm{~d}$ are fitted with calculated scattering patterns from a Mie-based code for coated spheres. As a first step, the parameter space of the simulation is carefully studied in the next section, before an attempt will be made to find parameters matching the time-binned experimental data.

\subsubsection{Simulation of scattering profiles from core-shell systems}

This section is dedicated to systematically study the influence of the variables causing and driving the observed variations in the scattering patterns which are indications of a refractive core-shell system. The basic dependencies found in these studies make it possible to simulate the measured difference profiles of Fig. $4.31 \mathrm{~d}$.

A core-shell Mie code developed by Shen [49] was used, which is based on the formalism presented by Bohren and Huffman [36] introduced in chapter 2.1.3. Several numerical difficulties and instabilities in the code from [36] could be overcome by incorporating different kinds of Bessel-functions [46]. Due to its structure as an executable file, which takes the input variables of the particle size, shell thickness and the refractive index of core and shell from a text file, the program could be easily included in the existent Matlab analysis.

Influence of the simulation parameters on the scattering profiles: In order to get a profound understanding of the contributions from all parameters of the simulation (radius, shell thickness and refractive index of core and shell), a large parameter range has been scanned with simulated scattering profiles. The obtained relationships are presented in Fig. 4.32 and detailed in this paragraph for the dominant parameters.

As a first step, the role of the absorption, i.e. the imaginary part of the refractive index $n^{\prime \prime}$ shall be discussed. In Fig. 4.32 a changing modulations are displayed for an increasing absorption in the outer shell with a thickness of $d=35 \mathrm{~nm}$ and a real-part-values of $n^{\prime}=0.97$. From green to yellow, $n^{\prime \prime}$ rises from 0.002 to 0.04, corresponding to a cross-section of 1 up to $20 \mathrm{Mbarn}$. The core is set to neutral values $m_{1}=1.001+i \cdot 0.04$ [60]. The modulation is only strong for very small $n^{\prime \prime}$ and vanishes completely towards neutral values, but no changes in the position of the modulation minimum occur. Thus, the low absorption in the outer part of the cluster can be interpreted as a necessary precondition for strong modulations. In other words, the light has to be able to deeply penetrate the cluster, be reflected and again leave 
a)

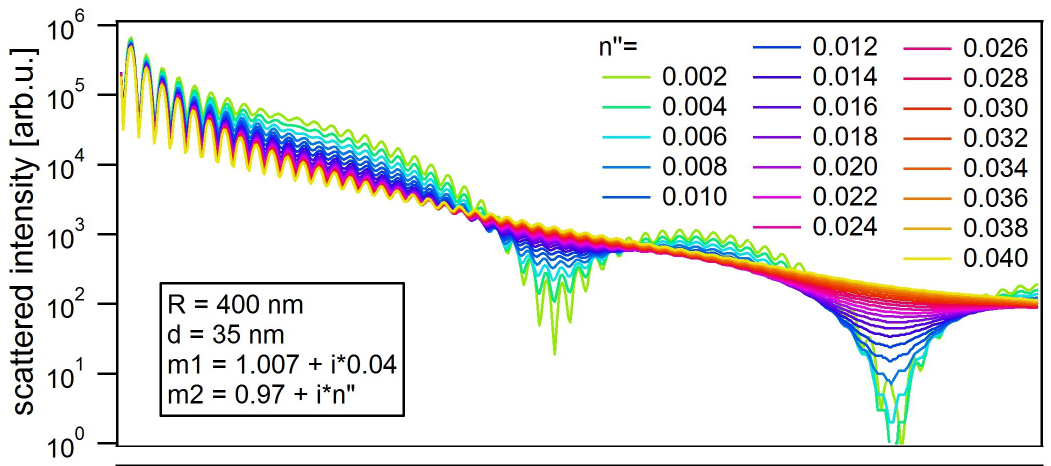

b)

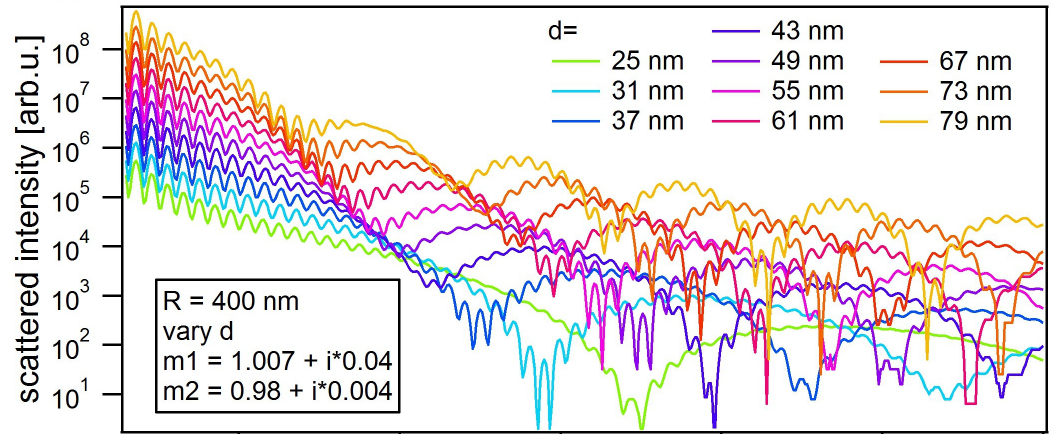

c)

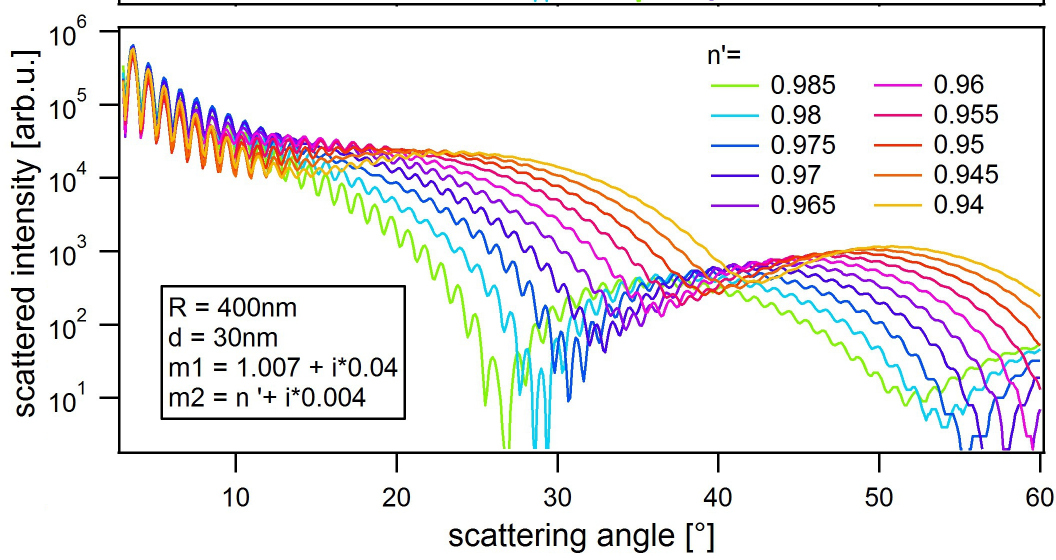

Figure 4.32: Systematic simulations of coated spheres. The parameters of the core are approximated by neutral xenon. The parameters of the shell are varied. a) The imaginary part corresponds to the absorption of the outer shell. An increasing value of $n^{\prime \prime}$ leads to a suppression of the beating pattern. b) The thickness of the shell changes the frequency of the superstructure. c) A decrease of the real part of the refractive index $n^{\prime}$ shifts the beating pattern towards higher scattering angles.

the cluster in order to interfere with waves which were refracted at the surface of the cluster and produce modulations. This is only possible if the absorption in the outer shell is low. It is important to note that virtually identical curves were observed in extended simulations even for drastic changes of the core absorption. As a direct implication, the discontinuity which leads to the modulations can not be related to the imaginary part of the refractive index. It has to be related to the real part of the refractive index.

Further, the thickness of the outer shell $d$ can be investigated. From principle considerations, an increase of $d$ due to the continuous ionization of the cluster would be expected. This is also further discussed in the next section, where the radial development of charge state distributions will be investigated.

A systematic study of changes in the modulations resulting from an increase of the shell 
thickness is given in Fig. 4.32 b. A rather low absorption of $n^{\prime \prime}=0.004$ was chosen, which corresponds to a cross-section of $2 \mathrm{Mbarn}, n^{\prime}$ was set to 0.98 . From bottom to top profiles for a shell with increasing thickness from 25 to $79 \mathrm{~nm}$ are displayed.

The increasing shell thickness leads to an increasing frequency in the oscillating superstructure. This behavior is in accordance with the basic law of diffraction, that a larger real space structure leads to a finer diffraction structure. At the same time, the observed modulations shift towards smaller scattering angles with increasing $d$. In the experimental difference profiles of Fig. 4.31, also a narrowing superstructure, but a shift to higher scattering angles is observed with increasing epd.

Furthermore, changes in the phase shift parameter $n^{\prime}$ are considered. Fig. $4.32 \mathrm{c}$ shows how the modulations change with decreasing $n^{\prime}$ from 0.985 to 0.94 from bottom to top, for $d=30 \mathrm{~nm}$ and again $n^{\prime \prime}=0.004$. While the width of the modulation lobes appears to be virtually unchanged by $n^{\prime}$ and only determined by $d$, the angular position of the modulation lobes clearly shifts towards higher scattering angles with decreasing $n^{\prime}$. This tendency is also observed in the experimental data.

It should be noted that simulations for variations of the real part ranging from values far above to far below unity also revealed shifts to higher scattering angles only for decreasing values of $n^{\prime}$. In addition, for a fixed neutral core value $m_{1}=1.007+i \cdot 0.04$, only $n^{\prime}<1$ yielded good matches to the experimentally observed developments. But as the shell thickness is not necessarily equivalent to the transition region from high to low charge states, which will be discussed in detail in the next section, also other core-parameters would be conceivable. This results in some uncertainty in fitting the measured difference profiles with simulated profiles.

Fits for experimental difference profiles: In Fig. 4.33 the averaged profile at lowest epd value and the difference profiles of the experimentally obtained average scattering patterns of $400 \mathrm{~nm}$ clusters are displayed on the left. On the right, profiles fitted to the observed structures are presented which were calculated with the Mie based core-shell code. For the core region, the optical constants of neutral xenon were used.

The fit parameters used for calculating the profiles in Fig. 4.33 are displayed in Fig. 4.35 as a function of time bin. The values fitted for the shell region reveal an increase of the thickness of the weakly absorbing outer shell from 25 to $48 \mathrm{~nm}$ while the imaginary part of the refractive index, which corresponds to the absorption, gradually decreases from $n^{\prime \prime}=0.0150$ to 0.0038 . At the same time, the real part of the refractive index $n^{\prime}$ drops from 0.970 to 0.928 .

In conclusion, simulated core-shell profiles, which match the experimentally obtained difference profiles, indicate the development of an outer shell with different refractive index compared to the core of the cluster. From the beginning of the interaction towards more developed intermediate states, the low absorbing shell increases in thickness. The resulting modulation, which is qualitatively similar to the one observed in the data, is directly connected with a discontinuity in the real part of the refractive index between core and outer shell. Towards later states, the real part of the outer shell decreases.

It should be noted that the current fit represents only one option in the highly dimensional parameter space and thus a certain degree of caution is obligued. For example a more pronounced growth of the shell could be balanced out to some degree by a lower value of $n^{\prime}$ if at the same time $n^{\prime \prime}$ was set slightly bigger. This is especially problematic, as no direct fitting of the intensity profiles can be executed due to the possible nonlinear efficiency of the scattering detector at small scattering angles (cf. appendix). 


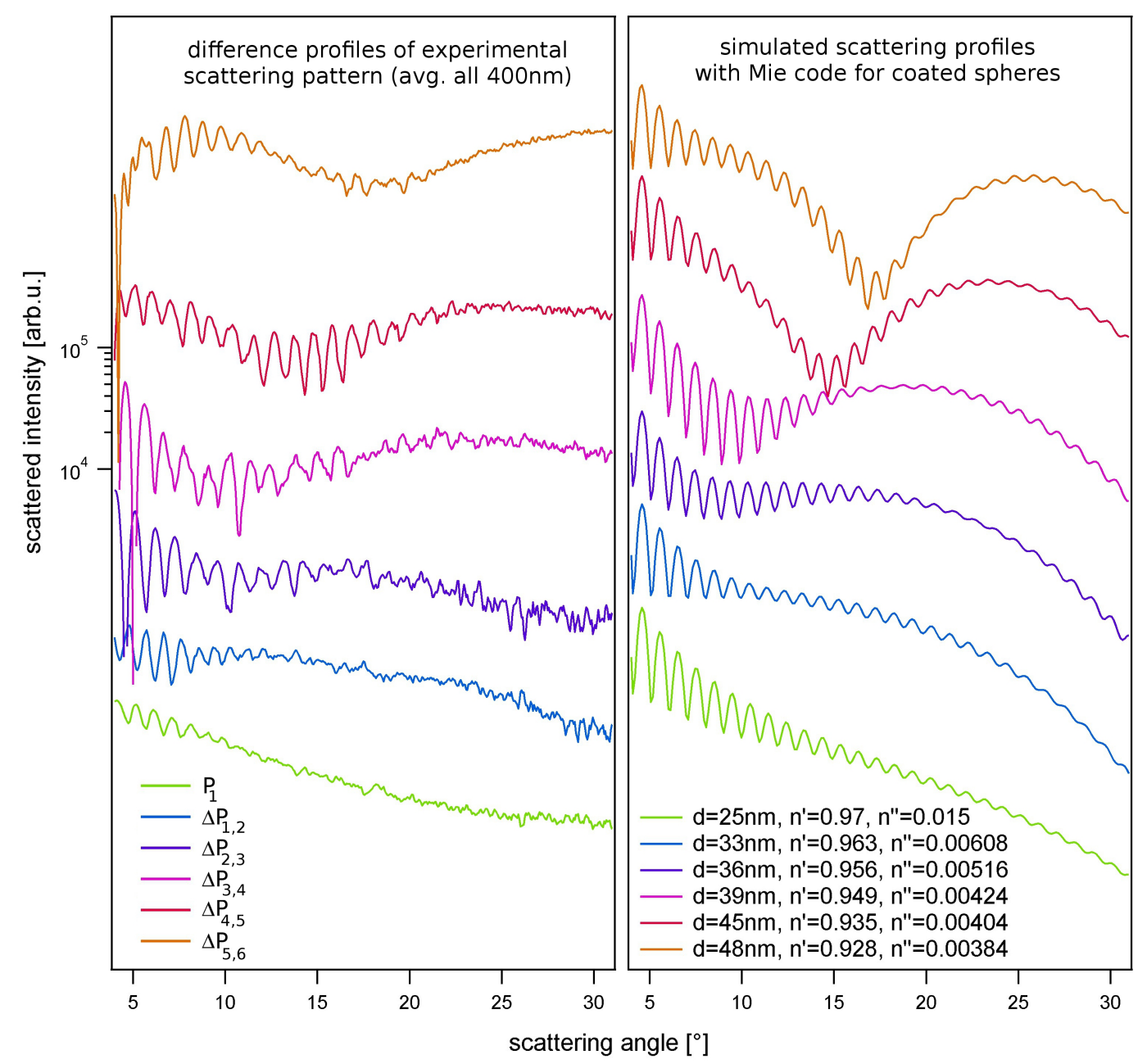

Figure 4.33: Simulating the time-binned experimental data with Mie theory for coated spheres. In the left panel the six time-binned profiles from Fig. 4.31 (five difference profiles and averaged profile with lowest epd) are displayed. Calculated profiles for a core-shell system with fit parameters as indicated in the legend yield reasonable agreement. See text for details.

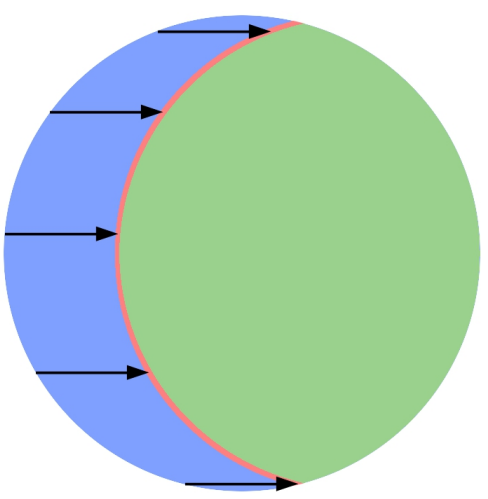

Figure 4.34: Asymmetric ionization dynamics, incidence of light from the left.
Further, the inhomogeneous ionization can not be considered to be a radially symmetric effect. A great difference in the ionization development will occur between the front and back side of the cluster with respect to the incoming beam. The side of the cluster facing the FEL will be more efficiently ionized. Instead of the picture of two spheres with different radii and properties placed concentrically one into the other, the real circumstances will appear more like the situation displayed in Fig. 4.34. The effects on the scattering patterns are out of the range of a Mie-based calculation and a current matter of discussion.

The derivation of the parameters from the fits, presented in Fig. 4.33, is complemented in the following section with considerations on the plasma characteristics and the ionization dynamics in the cluster during the interaction with the FEL pulse. In particular the search for a discontinuity 

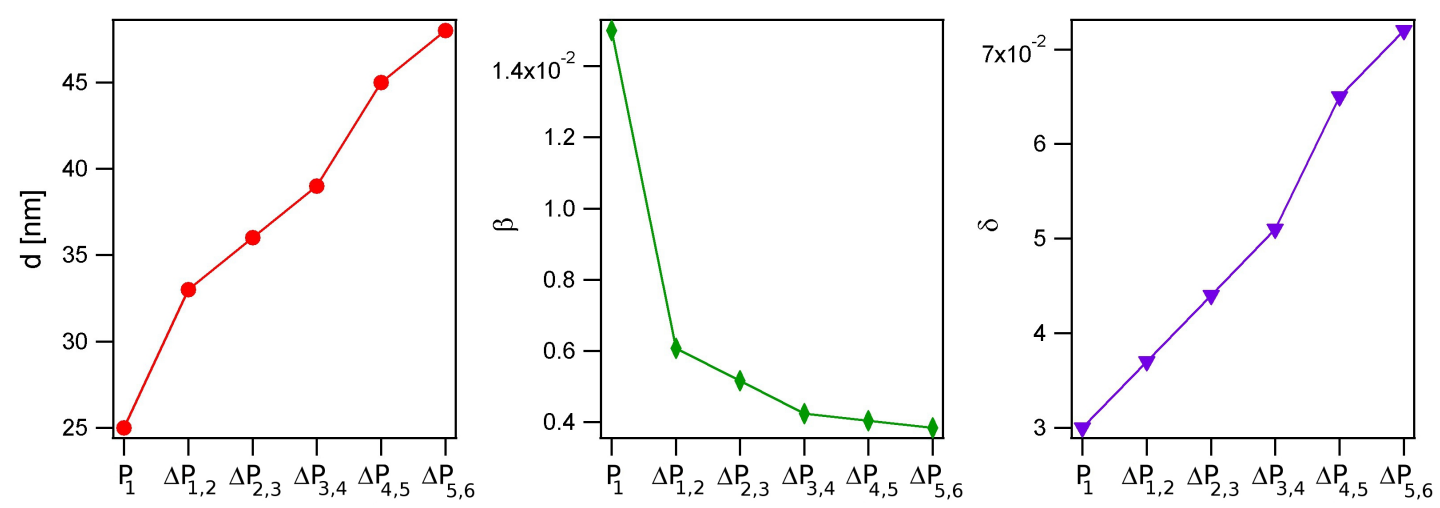

Figure 4.35: Fit parameters for the intermediate states defined by time-binning of the experimental data. The parameters were obtained by comparing with calculated profiles as shown above (cf. Fig. 4.33).

within the plasma, which appears to be counterintuitive at first sight, will be in the focus of the analysis.

In the next section the atomic properties of xenon are summarized and set into the context of the plasma environment. Changes in the structure of electronic states in the presence of neighboring ions and quasi-free electrons, as well as the direct contribution of the electrons to the optical properties will be discussed.

\subsubsection{Optical properties of a dense xenon nanoplasma}

In section 2.3.2 the effects of the plasma environment on atomic states have been introduced. Changes in the optical properties from the dense xenon nanoplasma are considered in this section. In particular three mechanisms play an important role:

- The vicinity of highly charged ions leads to a decrease of the ionization potentials. But also small changes in the relative energetic positions of atomic levels can occur [83, 82].

- The density of the quasi-free electrons will screen the charge from the nucleus, yielding again energy shifts of atomic levels [83].

- The electron cloud directly changes the real part of the refractive index [159].

In section 2.2.2, the absorption properties of atomic xenon and its ions for irradiation with $91 \mathrm{eV}$ photon energy have already been gathered from the literature. A summary of the absolute total and differential cross sections is given in table 4.3 .

These values reflect the response of electrons bound to free atoms or ions. As the values in the second column indicate a drastic step from high to low absorption with increasing charge state arises at $\mathrm{Xe}^{4+}$. However, as stated above, the optical properties of the large xenon clusters in the XUV pulses will substantially differ from the atomic values in table 4.3.

Energy level shifts in the cluster plasma: Already for the undisturbed cluster the high number of particles results in solid-state properties of the electrons instead of atomic 


\begin{tabular}{|l|l|l|l|l|}
\hline$q$ & $\begin{array}{l}\text { total } \sigma \\
{[\text { Mbarn }]}\end{array}$ & $\begin{array}{l}\sigma_{q \rightarrow q+1} \\
{[\text { Mbarn] }}\end{array}$ & $\begin{array}{l}\sigma_{q \rightarrow q+2} \\
{[\text { Mbarn }]}\end{array}$ & $\begin{array}{l}\sigma_{q \rightarrow q+3)} \\
{[\text { Mbarn] }}\end{array}$ \\
\hline \hline neutral & 23 & $<1$ & 12.5 & 10.5 \\
\hline $1+$ & 25 & 2 & 23 & 0 \\
\hline $2+$ & 22 & 4 & 16 & 0 \\
\hline $3+$ & 25 & 25 & 0 & 0 \\
\hline $4+$ & 200 & 200 & 0 & 0 \\
\hline $5+$ & $<2$ & $<2$ & 0 & 0 \\
\hline $6+$ & $<2$ & $<2$ & 0 & 0 \\
\hline $7+$ & $<2$ & $<2$ & 0 & 0 \\
\hline
\end{tabular}

Table 4.3: Total and partial xenon absorption cross-sections at $91 \mathrm{eV}$.

levels. As the clusters are bonded through the weak Van-der-Waals interaction, the distance to the next neighbor for an atom in the cluster is higher and the influence on the ionization potentials weaker than for other types of bond. Chemical shifts for rare gas clusters are measured to be on the order of $1 \mathrm{eV}[160]$.

A considerably more pronounced effect results from the plasma environment, which has been already discussed in section 2.3.2. The vicinity of other highly charged ions results in firstorder approximation only in an upshift of all energy levels $[83,82,34]$ and therefore in a suppression of the interatomic barriers. For the ionization potentials, considerable changes for the proximity of other ions of more than $30 \%$ have been predicted [82]. However, a higher order effect can be expected to slightly shift atomic levels in respect to each other.

Also, from the presence of a large number of quasi-free electrons, a change in the optical constants has to be expected [83]. The finite density of plasma electrons at the ionic cores causes a lowered attraction of the bound orbitals towards the core and thus results again in lower ionization potentials and presumably also in small relative changes of the energy levels. As a consequence, within a dense xenon plasma which exhibits spatial and possibly also temporal fluctuations of the local electric field, i.e. plasma screening, the energetic positions of the atomic resonances (cf. section 2.2.2) might be slightly shifted. Therefore, also resonant transitions slightly off the excitation energy, such as the $4 d-4 f$ transitions of $\mathrm{Xe}^{5+}$ and $\mathrm{Xe}^{6+}$ at 94 and $98 \mathrm{eV}$, could become accessible.

This consideration sheds new light on the findings in a previous light scattering experiment on single clusters with shorter pulses and lower power densities [32]. From the rising slope of the scattering profiles in this experiment, a tenfold absorption was derived. This increase could not be explained satisfactorily with the resonant cross-sections of $\mathrm{Xe}^{4+}$ alone. It appears less surprising considering the even larger cross-sections of $\mathrm{Xe}^{5+}$ and $\mathrm{Xe}^{6+}$ of up to $1000 \mathrm{Mbarn}$.

Phase shift from the electron cloud: The electron cloud also contributes directly on the optical properties by changing the real part of the optical constants. In plasma physics where mostly low density plasmas are investigated, the standard method to determine the electron density is to measure the phase shift of light propagating through the plasma [159]. Concepts from plasma physics as introduced in section 2.3.2 can also be used to estimate the direct contribution of the electron cloud in the case of the rather cold nanoplasma with supra-atomic density which is present in our experiments.

An assignment to different types of plasmas accounting for the density and temperature of 
the electrons has been introduced in section 2.3.2. The degeneracy parameter $\gamma$ reads

$$
\gamma=\frac{\hbar^{2}}{2 \cdot m_{e}} \cdot \frac{\left(3 \pi^{2} \cdot n_{e}\right)^{\frac{2}{3}}}{T_{e}}
$$

For FEL irradiated xenon clusters with several electrons per atom $\left(n_{e}=\bar{z} \cdot n_{a}=\bar{z} \cdot 1.7 \cdot 10^{22}\right.$ $\mathrm{cm}^{-3}$ ) and an electron temperature of about $20 \mathrm{eV}$, determined by the kinetic energy of the photoelectrons [80], a $\gamma$-value $>1$ arises, which indicates a quasi-metallic, strongly coupled plasma [161, 81]. Thus, following the example from [44], the cluster can be approximated by a metallic sphere. The response of the nanoplasma is given by the Drude-like dielectric function [50]

$$
\epsilon(R, \omega)=1+\chi_{0}-\frac{\omega_{p}^{2}}{\omega^{2}+i \cdot \omega \cdot \nu(R)} .
$$

The response of the bound electrons is condensed into the real-valued background susceptibility $\chi_{0}$. The second term $\omega_{p}^{2} /\left(\omega^{2}+i \omega \nu\right)$ describes the response of the electron cloud with the laser frequency $\omega$, the electron-ion collision frequency $\nu$ and the plasma frequency $\omega_{p}$. This complex term can be split up into real-valued and imaginary part by expanding the fraction with $\left(\omega^{2}-i \omega \nu\right)$

$$
\frac{\omega_{p}^{2}\left(\omega^{2}-i \omega \nu\right)}{\left(\omega^{2}+i \omega \nu\right)\left(\omega^{2}-i \omega \nu\right)}=\frac{\omega_{p}^{2}}{\omega^{2}+\nu^{2}}-i \cdot \frac{\omega_{p}^{2} \nu}{\omega^{3}+\omega \nu^{2}}
$$

The plasma frequency $\omega_{p}$, which was introduced in section 2.3.2 is a function of the electron density $n_{e}$. In a quasi-neutral plasma, $n_{e}$ equals the ion charge density and can be expressed as $n_{e}=\bar{z} \cdot n_{i o n}$ by an average charge state per atom of $\bar{z}$. Then the plasma frequency reads

$$
\omega_{p}=\sqrt{\frac{e^{2} \cdot n_{a} \cdot \bar{z}}{m_{e} \cdot \epsilon_{0}}} .
$$

The electron-ion collision frequency $\nu$ can be roughly estimated for the XUV spectral regime and plasmas with moderate temperature to be around $10^{16} \mathrm{~Hz}[44,87]$, at least one order of magnitude below the actual laser frequency of $1.4 \cdot 10^{17} \mathrm{~Hz}$. This value together with expression 4.7 can be substituted into the real part of the electron response of Eq. 4.6. By inserting all constant values $\left(n_{a}=1.67 \cdot 10^{28}\right.$, cf. next section) it reads

$$
\frac{\omega_{p}^{2}}{\omega^{2}+\nu^{2}}=\bar{z} \cdot 2.7 \cdot 10^{-3}
$$

As this value will be subtracted, an increasing electron density can thus be expected to lower the real part of the optical constants. This would be in agreement with the decreasing $n^{\prime}$ value found in the Mie fits in section 4.5.3.

From the Mie fits a decrease of the real part of the refractive index from $n^{\prime}=0.97$ to $n^{\prime}=0.928$ was obtained. The dielectric function $\epsilon$ is only another representation of the optical constants and can be calculated from the refractive index via $n=\sqrt{\epsilon}[50]$. By neglecting the absorption, i.e. the imaginary part, the dielectric function for both values of $n^{\prime}$ yields

$$
\epsilon_{1}=0.97^{2}, \epsilon_{2}=0.928^{2}-\Rightarrow \Delta \epsilon_{1,2}=7.97 \cdot 10^{-2}
$$

Now the assumption is made that the difference $\Delta \epsilon_{1,2}$ is given by an increase of the freeelectron density from 0 to $\bar{z}$ electrons per atom. Then Eq. 4.8 equals $\Delta \epsilon_{1,2}$, yielding an increase in electron density of up to 30 electrons per atom. This value is unlikely to be reached in the nanoplasma. Therefore the decrease found in the simulations with Mie theory for coated spheres can not only be attributed to the changes in the density of the quasi-free electrons. This indicates that also the bound electrons make a contribution to the real part of the refractive index which further decreases $n^{\prime}$. 
Phase shift from bound electrons: The approximation of a free electron dominated plasma and neglecting contributions from bound electrons is clearly not valid for xenon in the XUV range, where strong electronic resonances exist [159]. But not only the imaginary part of the optical constants, i.e. the absorption, will take extreme values, also the real part is strongly influenced, as the real and imaginary part are connected via the Kramers-Kronig dispersion relations (cf. section 2.1.1, equations 2.12).

The atomic scattering factor $f_{1}$ for the example of $X e^{3+}$ [159], displayed in Fig. 4.36, can give a striking example. The corresponding absorption cross-sections have been measured by Emmons et al. over a wide range in the XUV [65]. Plots of the cross-sections can be found in Fig. 2.11d in chapter 2.2.2. By using the Kramers-Kronig relations, $f_{1}$ was calculated by Nilsen [159]. A pronounced oscillatory structure with excitation energy can be observed in Fig. 4.36 with values changing from extreme positive to negative values (or vice-versa) within a small energy range. For the particular example, given in Fig. 4.36, the atomic scattering factor $f_{1}$ of -40 at the actual photon energy of $91 \mathrm{eV}$ yields an anomalous dispersion with a real part of the refractive index $n^{\prime \prime}$ of 1.06 .

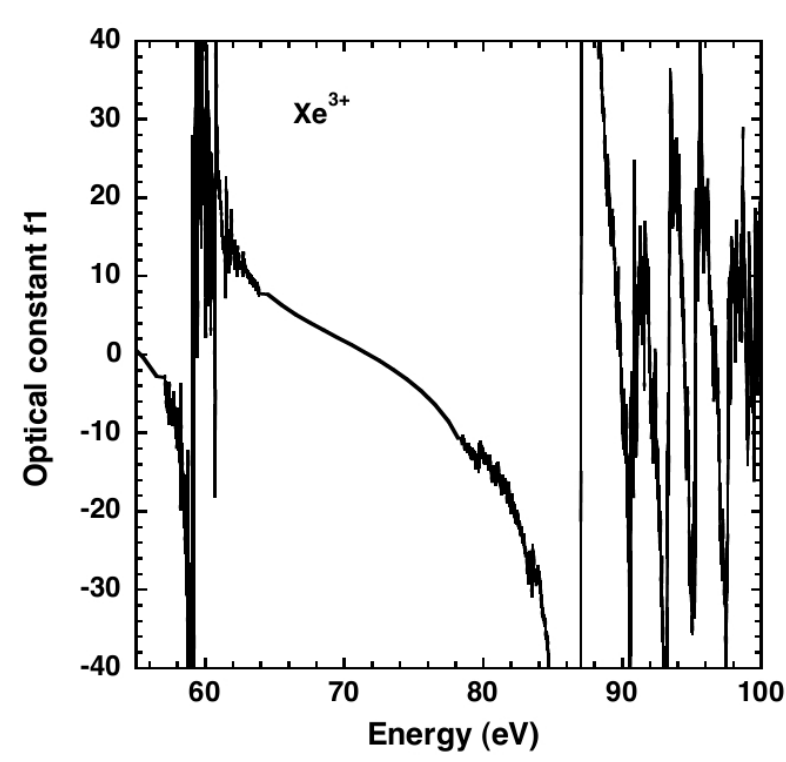

Figure 4.36: Atomic scattering factor $f_{1}$ for $\mathrm{Xe}^{3+}$ in the XUV regime[159].

To conclude in these considerations, the absorption cross-sections of xenon atoms and atomic ions yield a step at $91 \mathrm{eV}$ from high $\left(\mathrm{Xe}^{0,1+, 2+, 3+}\right)$ to low values $\left(\mathrm{Xe}^{5+, 6+, 7+, \ldots}\right)$ with a high absorbing intersection $\left(\mathrm{Xe}^{4+}\right)$. Screening effects of the nanoplasma might be able to make resonant transitions of $\mathrm{Xe}^{5+}$ and $\mathrm{Xe}^{6+}$ accessible for the actual excitation energy. The electron cloud contributes to the decrease the real part of the optical constants, but the influence of the bound electrons might dominate the real part of the optical properties in the vicinity of resonances.

In the next section, a simple model is utilized to trace the development of the radial charge state densities and the density of the quasi-free electrons in the interaction.

\subsubsection{Simulation of radial charge state densities}

In this section, the development of the changing degree of ionization along a radial path into the cluster are modeled with a simple Monte Carlo Simulation. The developed simulation studies the path of the photons into the cluster until they are absorbed in a certain depth. Therefore a simple one-dimensional model of the cluster with a sequence of photons propagating along a chain of atoms, or respectively ions has been developed. Statistical probabilities for the interaction between each atom and photon are applied, corresponding to the tabulated cross-sections of atomic xenon and its ions (cf. table 4.3). Important features of the simulation are explained in the subsequent paragraph and the Matlab code can be found in the appendix to this work. 


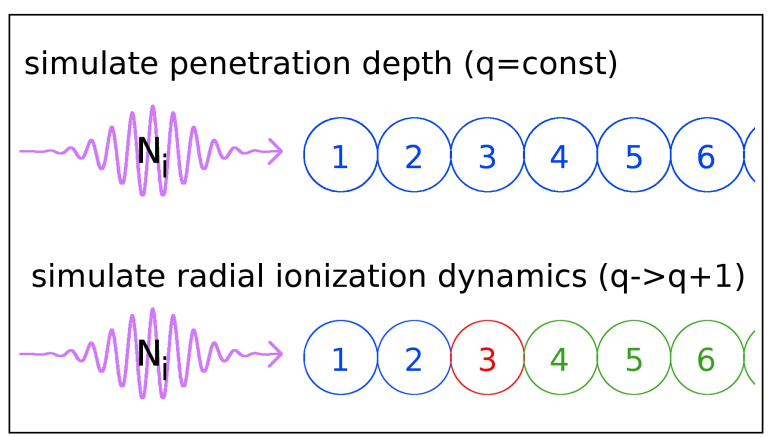

a)
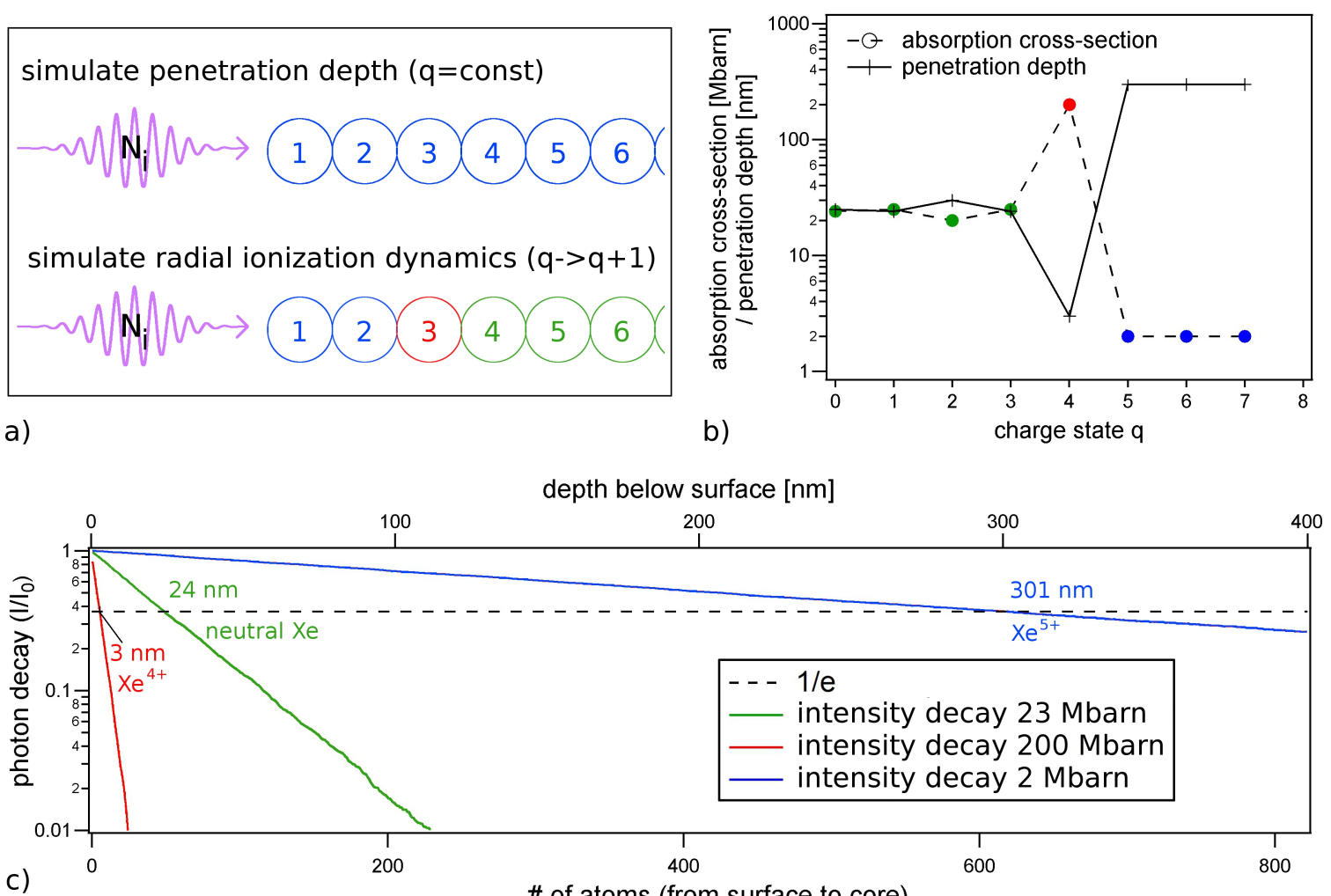

Figure 4.37: One-dimensional simulation of radial ionization dynamics: a) Photons propagate along a chain of atoms. Absorption is probed for every atom according to the charge-state specific absorption probability. As illustrated in the upper picture, for fixed charge states reproducing the penetration depth can be tested. The lower picture illustrates the dynamic simulation of the radial evolution of ionization. b) Absorption cross-section and penetration depth of the tabulated values for atomic xenon and its ions (cf. section 2.2.2). c) Simulated intensity decay for fixed charge states $0,4+$ and $5+$. The number of photons along the chain of atoms decays exponentially. The simulated penetration depths (decay to $e^{-1}$ ) match the tabulated values.

1D Monte Carlo model of ionization dynamics The behavior of a many-particle system can be modeled through a large number of simulation repetitions of statistical processes. This technique is referred to as Monte Carlo method [162].

The one-dimensional model of the cluster is schematically sketched in Fig. 4.37 a. The atoms are numbered from the surface to the core by $i=1 \rightarrow N_{a}$. The number of photons $N$ which fall on the geometrical cross-section of one xenon atom are calculated corresponding to the epd values which have been assigned to the averaged scattering profiles in section 4.5.2. The value for the geometrical cross-section of a xenon atom of $\sigma_{\text {geo }}=1.8 \cdot 10^{-19} \mathrm{~m}^{2}=1800 \mathrm{Mbarn}$ corresponds to a solid xenon density of $3.654 \mathrm{~g} / \mathrm{cm}^{3}$ [163], and respectively to a density of $n_{a}=1.67 \cdot 10^{28}$ particles per $\mathrm{m}^{3}$. From the resulting atomic radius of $r_{X e}=2.43 \AA$, the $400 \mathrm{~nm}$ cluster considered here exhibits $N_{a}=824$ atoms in a one-dimensional array.

To each atom, a charge state $q_{i}$ and an absorption probability $p_{i}=\frac{3}{2} \cdot \frac{\sigma}{\sigma_{g e o}}$ as the ratio of absorption cross-section to geometric cross-section are assigned (the pre-factor $3 / 2$ results from the one-dimensional approximation, see appendix). Therefore, the values for atomic xenon and its ions from table 4.3 are used. In cases where only limit values are given in table 4.3 - for example $<2$ Mbarn - they are included as 2 Mbarn. Charge states of $8+$ and higher are artificially set to zero cross-section. The total cross-sections are displayed for the charge states $0-8$ in Fig. 4.37 b. From these values the penetration depths $l_{a b s}$ can be calculated with Eq. 2.11. They are also shown in Fig. $4.37 \mathrm{~b}$. 
The $N$ photons are propagated sequentially through the chain of atoms, starting from the surface. For the $j$ th photon at the position of the $i$ th atom, random experiments probe the interaction. In particular, a random number rand between 0 and 1 is generated and compared to the absorption probability $p_{i}$. If rand $<p_{i}$ the photon will be absorbed, thus the charge $q_{i}$ is increased by 1 (for neutral, singly and doubly charged xenon, the probabilities for Auger decay and the resulting increase of 2 or even 3 charges are considered in the simulation). With a change in $q, p$ is also changed. Then the next photon starts from the surface. If the photon has not been absorbed at position $i$, the next atom $i+1$, or respectively ion, will be probed in a new random experiment.

In this simple, one-dimensional simulation, many effects are neglected, which can play an important role in the ionization dynamics. Namely elastic light scattering, recombination effects, charge redistribution in the cluster and changes in the cross-sections due to screening effects are not considered. However, Auger decay processes following the photoionization of neutral, singly and doubly charged xenon, are implemented by separate probabilities for the decay channels.

For the analysis of the ionization development, the charge state distribution $q_{i}(N)$ is tracked and in addition the position, at which each photon $N_{j}$ was absorbed, is recorded. Therefore, a population vector for each photon with $n$ entries is generated. Each atomic position passed by the photon is set to 1 , from the position $i$ on, where the photon was absorbed, the entries are set to 0 . By summing the population vectors up, the radial absorption behavior can be extracted.

The population vectors can also be used to check the reliability of the simulation by simulating the penetration depths. For this check, fixed charge states are simulated, i.e. all atoms start with the same charge state, and in case of a positive random experiment, the photon will be annihilated but the charge state remains constant. The characteristic penetration depths can be obtained from the simulation by summing up the population vectors, as displayed in Fig. $4.37 \mathrm{c}$ for neutral xenon, $\mathrm{Xe}^{4+}$ and $\mathrm{Xe}^{5+}$. The penetration depth (see also section 2.1.1) is defined by the distance over which the number of photons in average decreases by a factor of e. This threshold and the resulting penetration depths of 24, 3, and $301 \mathrm{~nm}$ are marked in the curves of Fig. $4.37 \mathrm{c}$. They match very well the calculated penetration depths of $l_{a}(q)$ from Fig. $4.37 \mathrm{~b}$ of respectively 25, 3 and $300 \mathrm{~nm}$.

Development of a low absorbing outer shell For the simulation of the charge state densities, $2.6 \cdot 10^{1}-2.6 \cdot 10^{3}$ photons have been propagated through the atomic chain, corresponding to epd values of $3 \cdot 10^{12}-3 \cdot 10^{14} \mathrm{~W} / \mathrm{cm}^{2}$. The results are presented in Fig. 4.38. The developing ionization from the outside into the cluster can be followed. A rather abrupt change from $\mathrm{Xe}^{3+}$ to $\mathrm{Xe}^{5+}$ with virtually no contribution from $\mathrm{Xe}^{4+}$ stems from the much higher absorption probability of $\mathrm{Xe}^{4+}$. With an increasing number of incident photons, the transition region between high and low charge states is driven further into the cluster.

From the analysis of the ion kinetic energies in the last chapter, a quasi-neutral plasma and therefore full screening of the ions by quasi-free electrons is expected. As a consequence the radial density of the quasi-free electrons can be calculated as the sum of the charge state distributions multiplied with the respective charge states. The number of electrons per atom for increasing depth below the plasma surface is displayed in Fig. 4.39 a. The electron density distribution reveals a rather steep gradient from approximately 6 to 2 electrons per atom within $50 \mathrm{~nm}$. This gradient is driven further into the cluster with increasing epd.

A similar development can be observed for the decay of the photons. The sum of the photon population vectors generated in the simulation is displayed in Fig. 4.39 b. A flat, low absorbing 


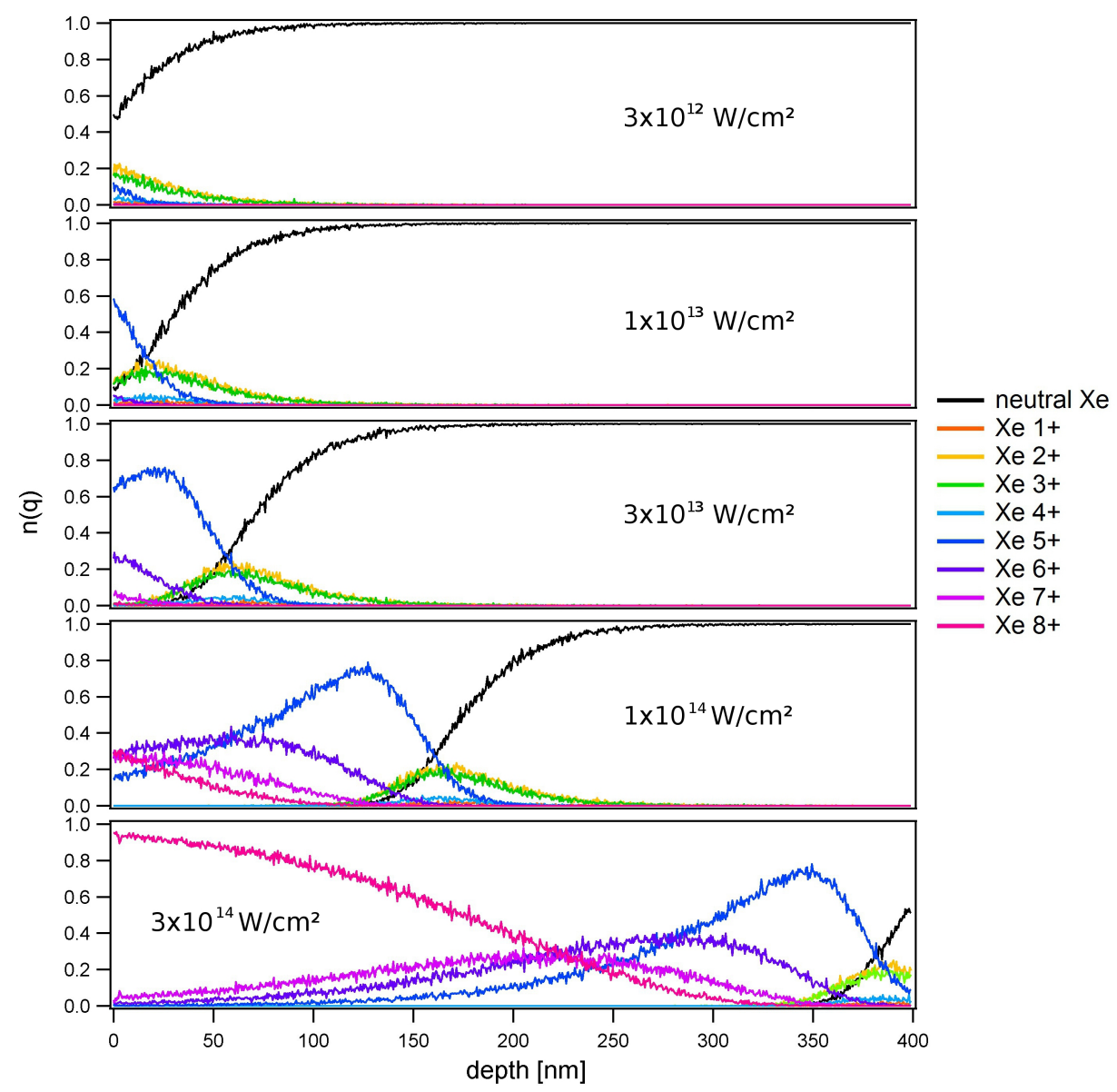

Figure 4.38: Radial development of the charge state densities simulated in a simple, one-dimensional Monte Carlo model. With increasing epd from $3 \cdot 10^{12}$ to $3 \cdot 10^{14} \mathrm{~W} / \mathrm{cm}^{2}$, corresponding to a number of incident photons per geometric cross-section rising from 26 to 2600, the lower charge states are driven into the cluster.

outer region and an inner, steeper and stronger absorbing region can be distinguished clearly.

The different slopes observed in the intensity decay of the photons (Fig. $4.39 \mathrm{~b}$ ) correspond to a step in the absorption from a rather low value in the outer shell to a higher neutral-like absorption in the core of the cluster. On the one hand, the systematic simulations of core-shell scattering profiles in section 4.5.3 revealed that the low absorption in the outer part of the cluster is a necessary precondition for strong modulations, but is not causing them. In fact, only a sharp edge in the real part of the optical constants, leading to pronounced phase shifts in the scattered waves, can create the prominent superstructure on the scattering profiles. On the other hand, with the transition between highly charged ions and lower charge states also a change in the real part can be expected.

In conclusion the simple simulations performed with the one-dimensional model yield the emergence of two regions within the cluster with different electronic properties. However, neither the values for the shell thickness resulting from the Mie fits section 4.5.3 can be reproduced, nor a sharp interface, necessary for prominent modulations, is found in the simulated radial ionization dynamics. The depth below the surface in which the large gradients in absorption and density of free electrons occur increases up to $330 \mathrm{~nm}$ for highest exposure power densities. This value is much larger than the shell thickness estimated from the Mie 


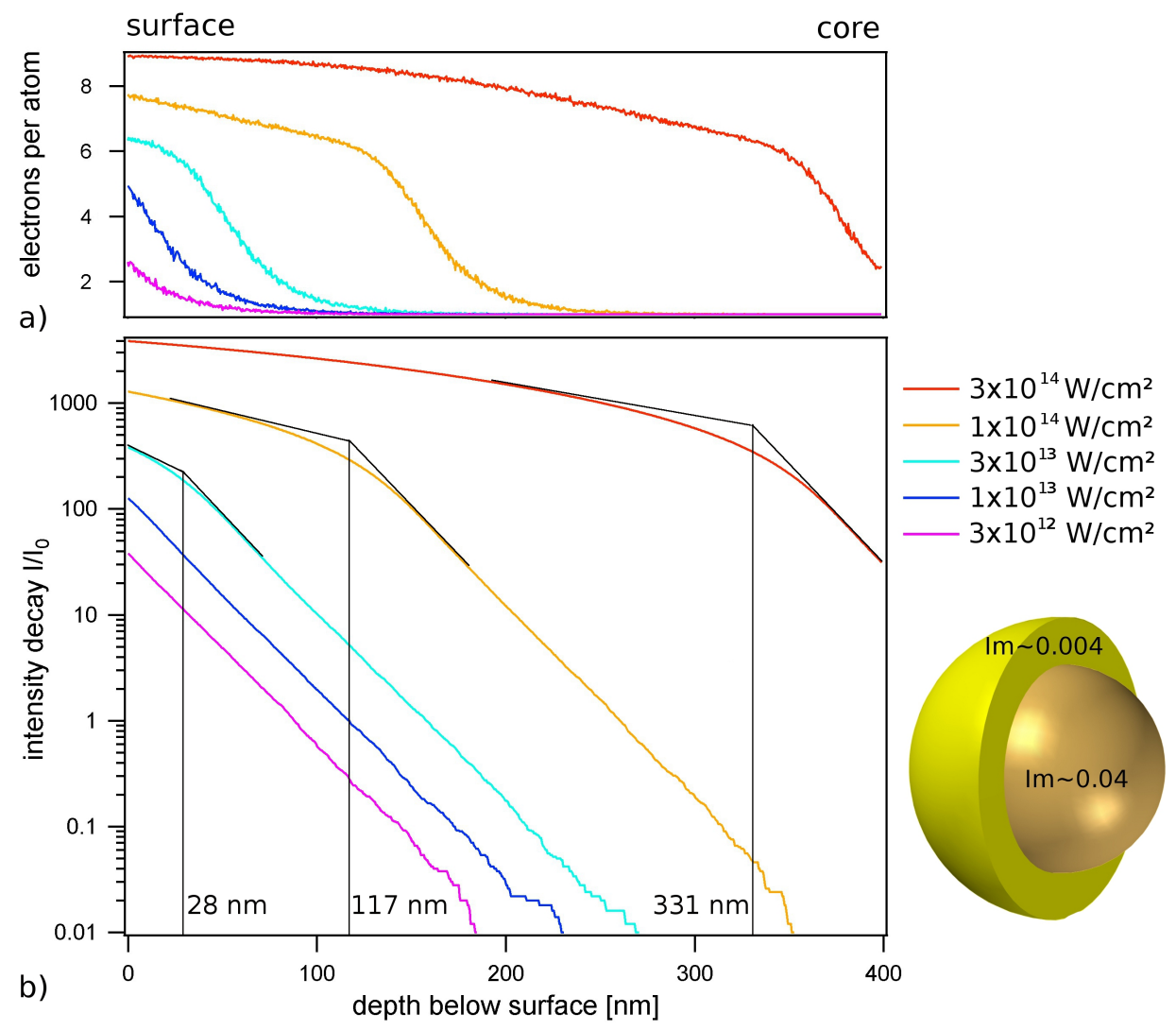

Figure 4.39: a) Number of electrons per atom as a decreasing function with depth below the surface. Full screening is assumed. The radial electron density reveals a steep gradient from six to two electrons per atom within $50 \mathrm{~nm}$. b) Development of intensity decay over distance from the cluster surface simulated in the Monte Carlo model. The transition from flat to steep slope indicates an increasing outer shell of up to $330 \mathrm{~nm}$ thickness with low absorption. The transition region with 70 to $100 \mathrm{~nm}$ is rather broad.

fits of $50 \mathrm{~nm}$. The transition region between both mostly linear parts with approximately 70 nm can not be considered a sharp edge.

The simple model used for this simulation can not be expected to yield perfect agreement with the experimental data. But on the other hand, most of the effects neglected in the simulation including recombination or charge transfer can be assumed to further smooth out the charge distributions.

However, two effects can be identified which could help to explain a more abrupt change in the optical properties and a smaller thickness of the outer shell.

For one, as discussed in the previous section the plasma environment may yield small shifts in the energy levels of the ions in the cluster. If the resonant features of $\mathrm{Xe}^{5+}$ and $\mathrm{Xe}^{6+}$ with very high cross-section were accessible for the $91 \mathrm{eV}$ radiation, the bends in the electron density distribution and the intensity decay curves would become more steep. Also the increase of the thickness of the outer shell would be slowed down with increasing overall absorption.

Second, scattering as a possible interaction process between photon and ion is neglected in this simple simulation. However, as discussed above a transition layer in the nanoplasma which is highly reflecting is a necessary precondition for the observed modulation in the data. If such a highly reflecting layer is present in the nanoplasma, the effect of scattering 
will become important. The photon density in a greater depth than the reflective layer would decrease, which results in less ionization in the core. Again, the increase of the shell thickness would be slowed down.

Explaining the sharp interface within the nanoplasma appears to play a pivot role for understanding the emergence of modulations in the scattering pattern. Based on the radial distributions of charge states and quasi-free electrons, an attempt will be made in the next section 4.5.6, to indicate possible explanations for the emergence of an otherwise contraintuitive discontinuity in the nanoplasma.

\subsubsection{Conclusions for the scattering data and discussion}

Characteristic modulations in all scattering patterns of large xenon clusters reveal a clearly visible epd dependence but only a weak size dependence. Initial considerations lead to the conclusion that the beating pattern is a signature of a refractive index core-shell system with a sharp interface within the nanoplasma. Difference profiles from averaged scattering patterns were analyzed in order to get access to the contributions of the transient stages in the development.

A Mie-based simulation for coated spheres was used for the analysis of the difference profiles. The Mie simulation incorporates six variables: radius, shell thickness and refractive index for both regions, each consisting of real and imaginary part. Systematic studies show that a discontinuity in the real part causes the emergence of a beating pattern, while an increasing absorption in outer shell will suppress the beating pattern. Changes of the shell thickness alter the frequency of the beating pattern, while variations in the real part of the refractive index will mainly shift of the superstructure in respect to the scattering angles.

The experimental difference profiles were fitted with simulated patterns. The refractive index of the core was set to neutral xenon, the thickness and refractive index of the shell served as fit parameters. From the initial towards later states of the plasma, the parameters appear to change in the following fashion:

- The thickness of the outer shell increases.

- The absorbtion in the outer shell decreases.

- The real part of the refractive index in the outer shell decreases.

These developments could be further enlightened by considering the special ionization properties of xenon at $91 \mathrm{eV}$ excitation energy and the radial evolution of the charge state distributions. While the lower charge states of xenon exhibit a strong absorption, an outer shell with increasing thickness will be highly photo-ionized and thus only weakly absorbing. The decreasing real part of the refractive index can be at least partially attributed to the increasing density of quasi-free electrons in the outer shell.

The observed changes in the time-binned scattering profiles have to be considered very surprising. For one, the profiles are obtained by averaging over many single patterns. Further, the difference profiles are the integration over a time bin, i.e. a certain range of transient interstages. Both effects are expected to suppress the beating pattern, therefore even more extreme transient values of the optical constants in the plasma are conceivable. Definitely the most puzzling detail consists in the radical change of the real-valued $n^{\prime}$ on a very short length scale which is necessary for explaining the presence of modulations. 
Speculation about the origin of highly reflecting transition layer within the plasma: The proposed explanation is based on mainly two effects.

For one, the radial charge state density distributions simulated in section 4.5.5 result in radially changing local electric fields, that is a radially changing plasma screening. In particular the radial distribution of quasi-free electrons exhibits a steep gradient in the transition region towards lower charge states, as displayed in Fig. 4.39 a.

Secondly, as the example of $\mathrm{Xe}^{3+}$ in Fig. 4.36 shown in section 4.5.4 demonstrated, also the real part of the refractive index undergoes drastic changes in the vicinity of strong absorption resonances.

These two results combined could be taken as an indication towards the following idea: The drastic oscillations of $\mathrm{Xe}^{3+}$ in $n^{\prime}$ on the energy scale are transformed by the radial plasma screening gradient induced by the quasi-free electrons into spatial oscillations of $n^{\prime}$. In other words, the high reflectivity is due to the development of an oscillatory plasma structure which could be termed a transient plasma multilayer. A sketch of the concept is given in Fig. 4.40.
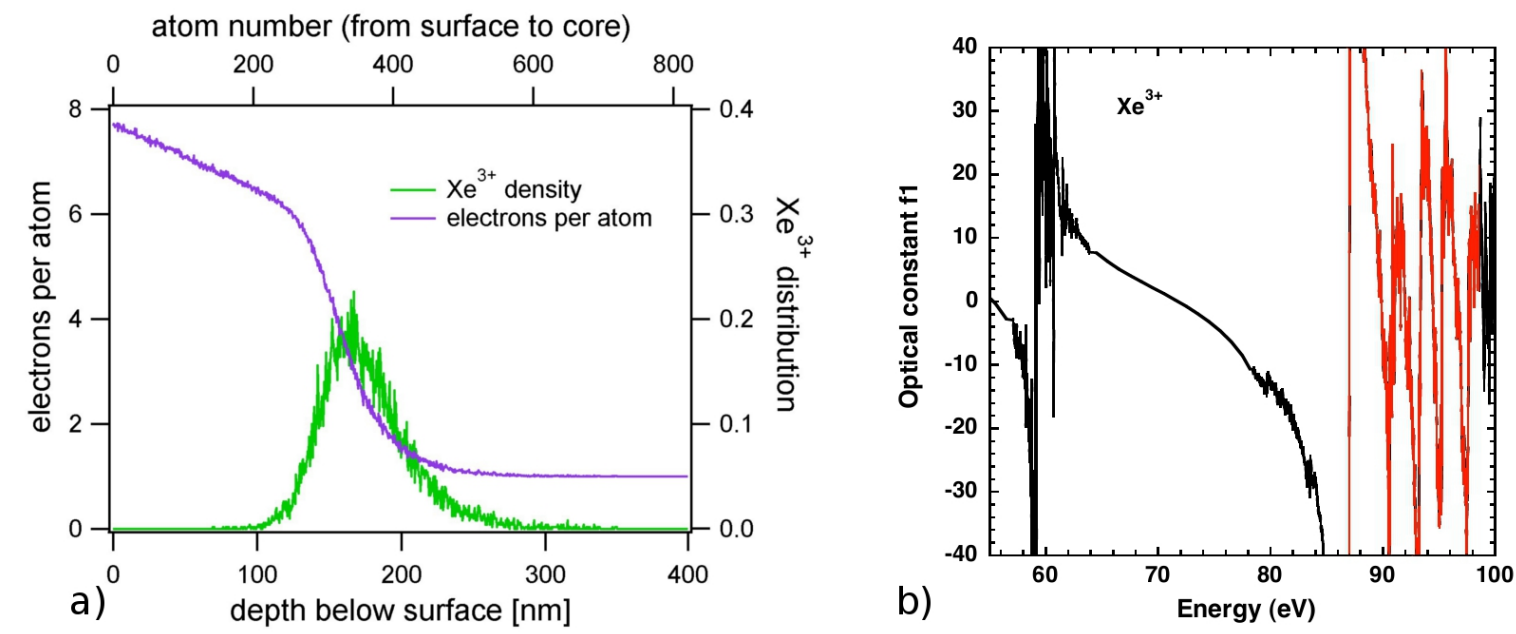

Figure 4.40: A strong screening gradient resulting from the radial density distribution of the quasifree electrons falls together with the radial distribution of $\mathrm{Xe}^{3+}$. For this charge state, strong oscillations of the real part of the refractive index close to the excitation energy have been reported [159]. Both effects in combination might transform the strong oscillations of $n^{\prime}$ within a short energy range into spatial oscillations of the real part of the refractive index. This would lead to a multilayer-like effect several tens of nanometers within the plasma. 


\section{Chapter 5}

\section{Summary and Outlook}

In this work the ionization and plasma dynamics of single large xenon clusters induced by intense XUV pulses from the free-electron laser FLASH have been investigated. Using coincident ion spectroscopy and light scattering allowed gaining insight into different time scales of the interaction and have found to be sensitive on different aspects of the cluster dynamics. A key aspect of the analysis of the single shot data was using the scattering patterns of individual clusters to sort the data for well defined systems in size and shape as well as in exposure power density.

The information from the single cluster scattering patterns on shape, size and abundance of the clusters led to new insight into the cluster growth process and experimental conditions for producing giant clusters up to microns in diameter. Their emergence appears to be tied to the pulsed operation of the cluster jet, possibly from the creation of clusters out of the liquid phase in the closing of the valve. With increasing cluster size, more frequently a grainy structure of the particles was found, existing in parallel to spherically shaped clusters. For radii above $200 \mathrm{~nm}$, only grainy hailstone-like clusters appeared.

In the size-sorted ion spectra of large xenon clusters with several hundreds of nanometer radius, ions with a narrow kinetic energy distribution were observed, paired with a characteristic linear increase of the central energy on the charge state. These findings indicate, that only the outermost surface layer of the cluster contributes to the ion spectra. Charge separation on the surface of an almost neutral nanoplasma could be identified as the leading mechanism for ejecting the ions. The largest part of the otherwise fully screened, dense and rather cool nanoplasma recombines to neutral atoms and thus disappears from the view of ion spectroscopy.

From the the scattering patterns of the same clusters information on the transient structure of the nanoplasma several tens of nanometers beneath the cluster surface could be gained. Power density dependent modulations in the scattering profiles of more intense hits were found, which depend only weakly on the cluster size. These modulations indicate a transient refractive core shell system, evolving on the $100 \mathrm{fs}$ time scale of the interaction with the FEL pulse. Insight into changes of the thickness and the refractive index of the outer shell during the evolution of the nanoplasma was gained by fitting the scattering profiles with Mie theory. The analysis of the fits indicates that both, the special electronic properties of xenon at the excitation energy of $91 \mathrm{eV}$ and the quasi-free electrons in the ultrafast evolving nanoplasma together are responsible for the emergence and the development of the modulations in the scattering patterns. 


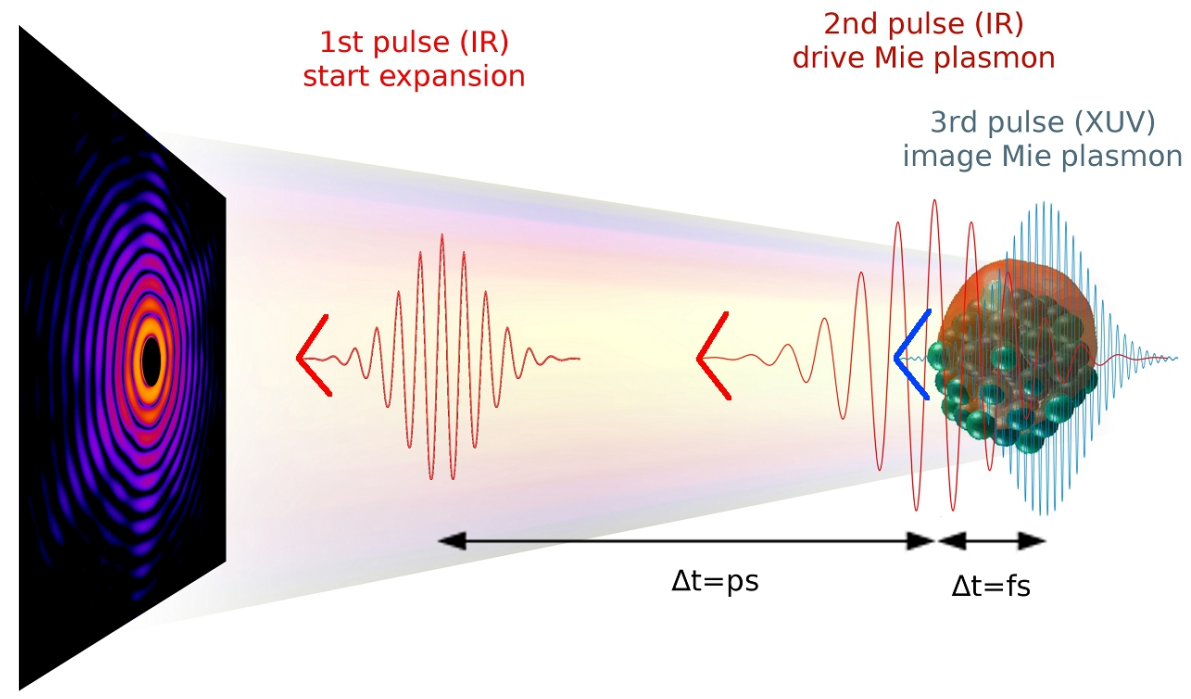

Figure 5.1: A large amplitude collective motion of the electron cloud, also referred to as Mie plasmon, can be enforced in the nanoplasma with an infrared laser pulse, if the cluster is expanded up to a critical density. Three pulses are needed for imaging a Mie plasmon. One laser pulse initiates the expansion of the cluster. The subsequent IR pulse excites the Mie plasmon in the expanded cluster and a coincident XUV pulse images the highly excited plasma.

These results emphasize the opportunities of single cluster light scattering experiments, to deliver fascinating insight into transient electronic states in the clusters. Further experiments are planned for the future combining the method of coincident single cluster imaging and ion spectroscopy with pump-probe techniques. With infrared laser pulses a nanoplasma with different degrees of inner ionization can be prepared and imaged by a shortly delayed XUV pulse. With intensities of the IR laser above $10^{15} \mathrm{~W} / \mathrm{cm}^{2}$ even average charge states of $\mathrm{Xe}^{26+}$ can be generated [88], making the cluster fully transparent for ionization with XUV light. In this case, mainly the cloud of delocalized electrons in the plasma is expected to contribute to the scattering pattern.

In addition to the influence on the scattered light from bound and uncorrelated moving quasifree electrons, collective motions of the electron cloud can be studied. A Mie plasmon, i.e. a large amplitude collective motion of the electron cloud can be created in the nanoplasma with an infrared laser pulse, if the cluster is expanded up to a critical density. As illustrated in Fig. 5.1 a third laser pulse can be used to initiate the expansion of the cluster. The subsequent IR pulse excites the Mie plasmon in the expanded cluster and a coincident XUV pulse images the highly excited plasma. The proposed pump-probe experiments promise to help disentangling the complex interplay of bound and free electrons in the light-matter interaction.

Detailed insight into the influence of the plasma environment on the specific atomic structure in highly excited super dense plasmas could be gained by imaging single large clusters of different target materials with different on- and off-resonant excitation wavelengths. This prospect is in particular interesting with the perspective of seeded FEL sources such as FERMI [114] or sFLASH [115], where exact photon energies can be produced and scanned. In this context, the investigation of large individual clusters with scattering methods holds the promise to yield insight into unexplored short-lived phenomena of finite plasmas. 
Appendix 



\section{Detector calibration}

The scattering pattern from single clusters analyzed in section 4.5 have been obtained with a large area detector which allows for spatially resolved measurement of the scattered light. This scattering detection system has been introduced in section 3.2.3.

For correct interpretation of the scattering patterns, it is necessary to consider the efficiency flatness of the scattering detector, i.e. to analyze if the detection efficiency changes for different positions on the detector. Further, it is important to know to which extend the detector signal is a linear function of the scattered light falling on the detector over the dynamic range in this experiment. These questions will be considered in some more detail within this paragraph.

The discussion will be based on a data set from a pump-probe experiment using an additional infrared laser pulse $(\lambda=800 \mathrm{~nm})[158]$. The experiment was carried out using the current setup as described in section 3.2. The infrared laser alone is not able to produce a scattering pattern of the single clusters due to the long wavelength. However, it produces a high amount of fluorescent signal, which can be of use for this discussion as its emission is approximately isotropic.

Detector profiles of single shot images produced with a single IR-pulse, which show different amounts of fluorescence are displayed in Fig. 2. The profiles are already corrected for the flat detector by $1 /(\cos \theta)^{3}$ (cf. section 4.5.1). Assuming a constant detection efficiency over

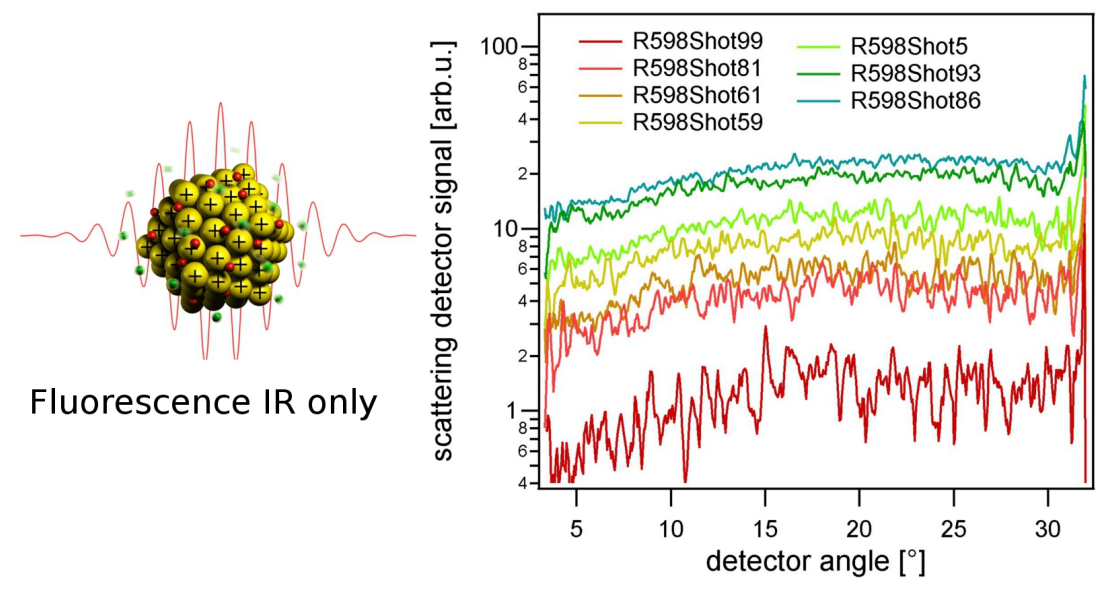

Figure 2: Fluorescence from large xenon clusters in single IR pulses. The signal on the scattering detector is due to strong fluorescence. This radiation is approximately isotropic and can be used to investigate the detection efficiency over the detector area. The radial profiles of the fluorescence (corrected for the flat detector) reveal a decreasing efficiency towards smaller angles. 

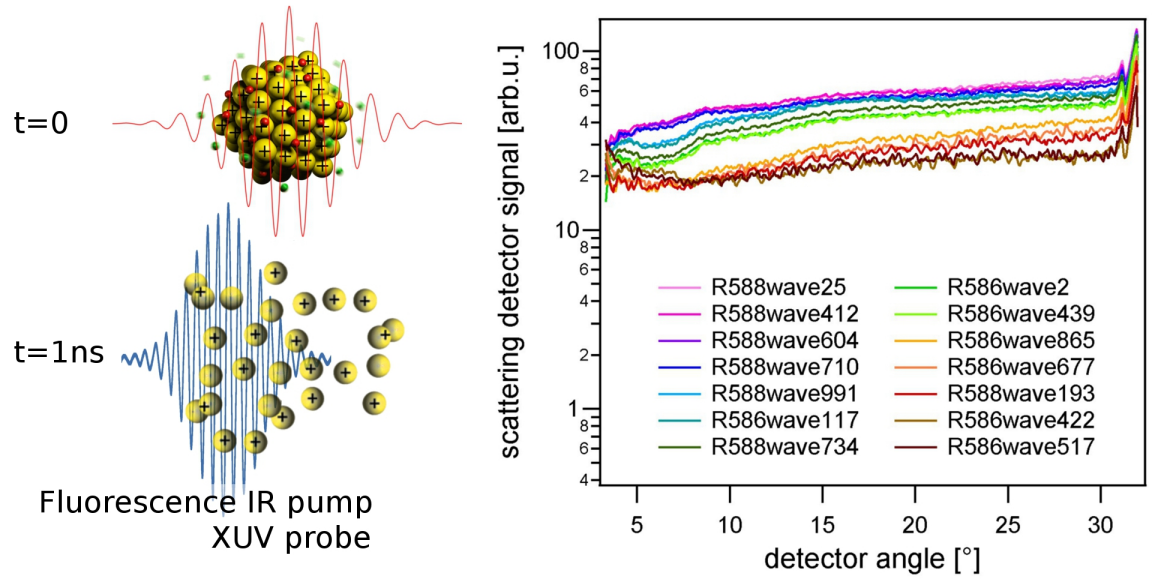

Figure 3: Higher fluorescence intensities are reached in an IR-pump-XUV-probe experiment for very long delays in the ns range. The curvature of the fluorescence profiles is very similar for all intensities.

the whole detector, the profiles should also be constant. In contrast, they exhibit a decrease towards smaller detection angles, however, the curve progressions appear very similar for all single shots.

Further, some single shots from the IR-pump-XUV-probe experiment [158] are displayed in Fig. 3. For very long delays between infrared and XUV laser pulses exceeding $1 \mathrm{~ns}$, the single shot images from the scattering detector show no scattering signal when the clusters which were pumped by the infrared pulse are already destroyed. However, many single shot images reveal a large fluorescence signal, as indicated by the profiles in Fig. 3 The curve progressions are similar to the IR-only profiles in Fig. 2, despite some straylight from the XUV pulse at small detection angles.

The decrease of the fluorescence signal towards smaller detection angles can be attributed to detector regions with decreased efficiency. Fig. 4 a shows the single shot image corresponding to the most intense profile in Fig. 2 with some irregularities of the detector indicated by
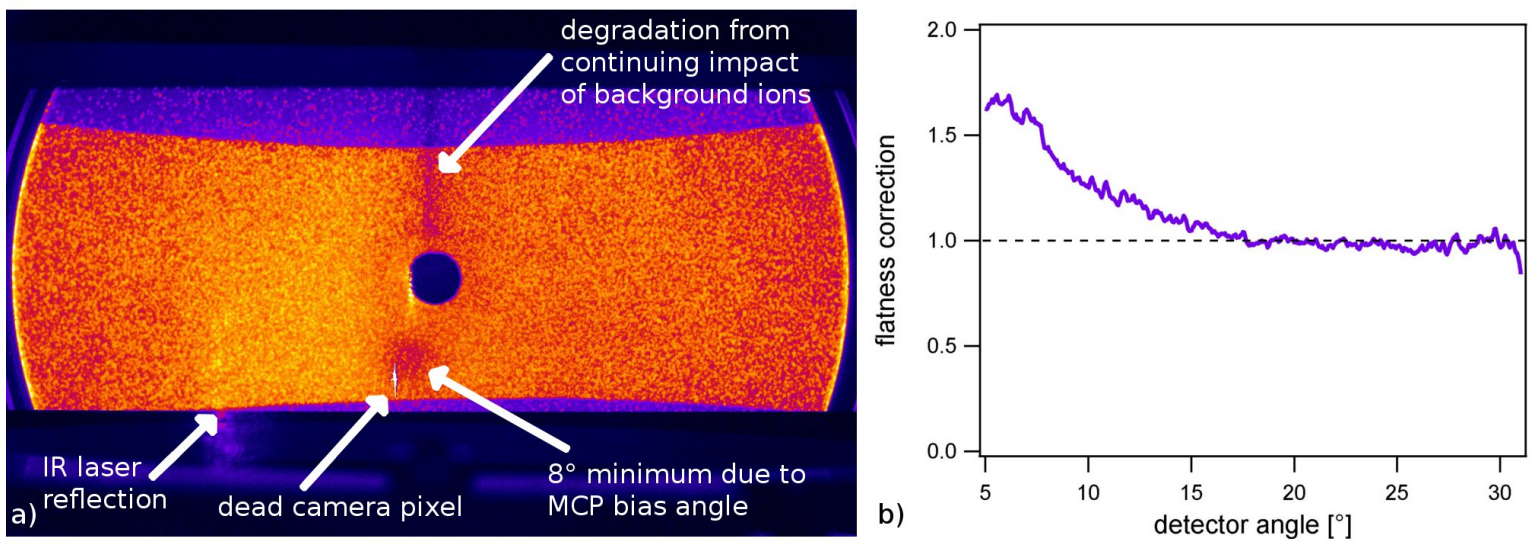

Figure 4: a) Single shot image with high fluorescence intensity on the scattering detector. The single shot corresponds to the most intense profile in Fig. 2. Irregular areas are indicated with arrows, see text for details. b) Correction curve for decreased efficiency at small scattering angles, calculated from the averaged fluorescence profiles in Fig. 2 and Fig. 3. 
arrows. A stripe of decreased signal can be found above the center hole of the detector. At this location ions from background gas in the chamber can be found, if the MCP is not gated (cf. section 3.2.3), which are produced by the exiting FEL beam and pulled towards the MCP. Their continuing impact appears to induce a degradation effect in this detection area. Further, on the lower left side of the detector's center hole, a weak spot can be found. The decreased efficiency is not due to degradation of the detector, but a geometric effect. The MCP channels are tilted to the surface normal by an angle of $8^{\circ}$, as described in section 3.2.3 in order to enhance detection efficiency. As the clusters in the interaction region can be considered a point source of light, the scattered or fluorescent photons in a certain direction will deeply penetrate the channels before they are detected, resulting in a decreased gain of the signal. In accordance to the bias angle of the MCP channels, the efficiency minimum is observed under an angle of $5-12^{\circ}$. This geometric effect occurs in all kinds of applications using spatially resolving MCP-based detectors combined to a point source of emission.

Two further irregularities of the detector images are indicated in Fig. 4 a: Due to a direct hit with the infrared laser, the out-of-vacuum CCD camera has several dead pixels in a region close to the $8^{\circ}$-minimum. An artifact on the images which only occurs in the presence of the infrared laser is an extended bright area on the left side of the center hole which results from a reflection of the infrared laser falling directly on the CCD. Nevertheless, it appears that the overall signal on the left side of the detector is stronger than on the right side. To what extend this effect results from the IR reflection or an actual difference in detector efficiency is not easy to decide.

In conclusion, deviations from a constant spatial efficiency of the scattering detector can be accounted for by calculating a correction curve from the profiles in Fig. 2 and 3. This curve is displayed in Fig. 4 b. In accordance to the above described observation of decreased efficiency towards smaller observation angles, also the correction curve deviates from the constant value of 1 for angles below $15^{\circ}$ and rises up to a factor of 1.7 .

It is important to note that within the analysis of the scattering patterns from single clusters in section 4.5, this correction has not been applied. Instead for determining the scattering profiles, the region of the detector was restricted to the right side, excluding all irregularities discussed above.

The more critical issue for the interpretation of the epd-dependent changes found in the scattering patterns of similarly sized individual clusters (cf. section 4.5) is the response function of the detector with increasing incident scattering signal. Again the fluorescence light produced with the infrared laser can be used for the discussion. In Fig. 5 the integrated signal from fluorescence light found in the signal from the ion detector is presented as a function of average signal from the scattering detector for every single shot of the data set which also contains the hits presented in Fig. 2. The very weak slope (dotted line) in the first part of the graph corresponds to the "empty" shots, where no cluster was hit and only statistical fluctuation for example due to straylight influence both scattering detector and ion detector in a characteristic manner. For hits, i.e. shots which contain the signal from at least one cluster, the light signal on the ion detector increases approximately linearly with the average signal from the scattering detector which is indicated by the dashed line. However, several shots clearly deviate from the dashed line, probably due to different positions of the clusters under the aperture of the ion spectrometer. This effect appears to be particularly strong in this data set. A reason therefore might be found in the fully opened skimmer slit for the measurement with the infrared laser, which allows for larger fluctuations in the cluster position.

However, a definite answer to the question, whether the signal from the scattering detector scales linearly with the actual scattering intensity or not over the whole dynamic range can 
not be given, mainly for two reasons.

First, the signal on the scattering detector from elastic scattering of individual large clusters is up to a factor of 4 higher than the maximal fluorescence signal obtained in the IR-XUVpump-probe measurements. Therefore, no information is available for the in particular critical higher intensity range.

Second, only data sets with lower fluorescence signal can be analyzed for the light peak in the ion detector. The intensity of the fluorescence light for example from the pump-probe experiment with long delays as presented in Fig. 3 is high enough to saturate the MCP in the ion detector. The early saturation of the light peak in the ion spectrometer is a characteristic feature of the fluorescence light and due to the fact that the fluorescent light is emitted approximately isotropic. In contrast, only for the largest clusters imaged in this thesis with a single XUV pulse at highest epd values, the light peak in the ion spectrometer is clearly saturated.

In view of the above discussed uncertainties, the epd values which have been assigned to the ion spectra and scattering patterns of single clusters in sections 4.4 and 4.5 should only be considered a guide.

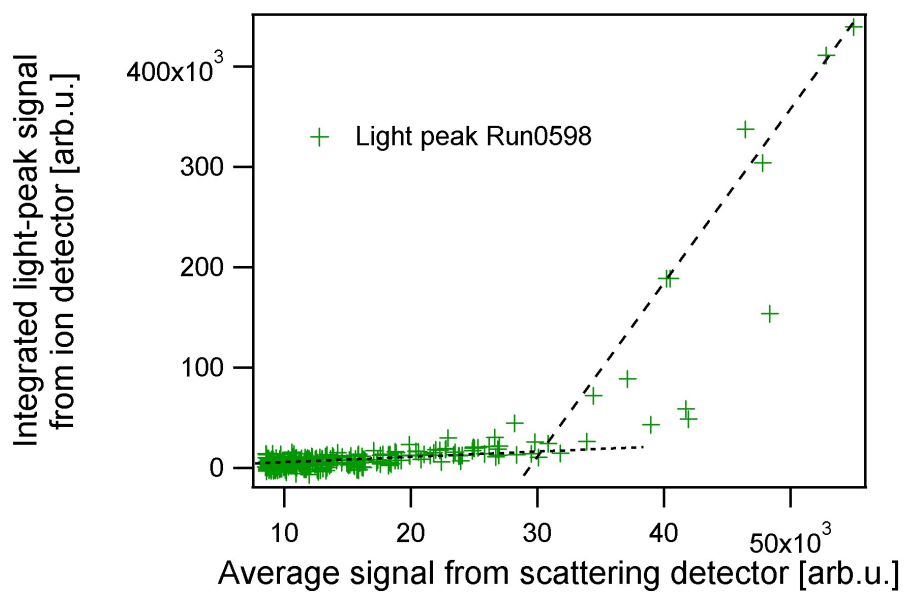

Figure 5: Integrated signal from the light peak of the ion detector as a function of the average signal from the scattering detector. The single shots are taken from the data set discussed in Fig. 2. A weak slope in the first part of the graph indicated by a dotted line corresponds to shots where no cluster was hit. Statistical fluctuations due to straylight influence both scattering detector and ion detector result in a weak positive slope. For shots containing signal from clusters, the light signal on the ion detector increases approximately linearly with the average signal from the scattering detector, as indicated by the dashed line. Several shots deviate from the dashed line due to different positions of the clusters under the aperture of the ion spectrometer. 


\section{D Monte-Carlo simulation of light penetration}

In section 4.5.5 the radial ionization dynamics of large xenon clusters irradiated with $90 \mathrm{eV}$ pulses have been traced in a one-dimensional model simulation. The details of the model and the realization in a simulation algorithm have been discussed in section 4.5.5. The source code of the simulation which has been developed for Matlab is presented here. Subsequently a factor of $3 / 2$ in the absorption probability will be demonstrated.

The code starts with a prefix containing a number of definitions and input parameters, such as the cross-sections which are taken from tabulated values. The prefix is followed by the static simulation of the penetration depth for a distinct, fixed charge state. Finally, the radial ionization dynamics are simulated starting with neutral atoms.

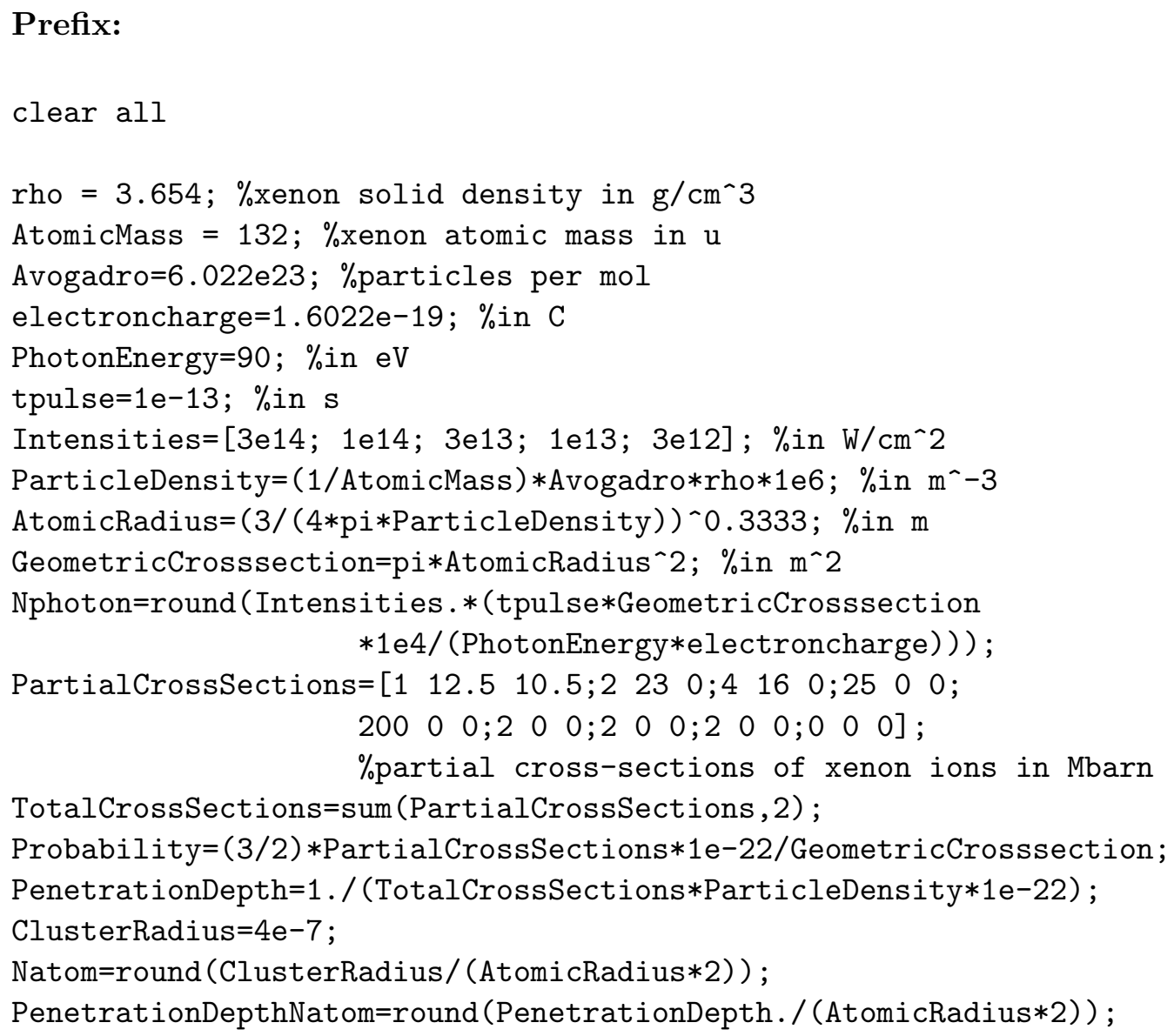




\section{Simulation of penetration depths:}

$\% \% \%$ Static simulation of penetration depths for neutral xenon, $4+$ and $5+$ PenDepthMatrix=zeros (Natom, 3);

for $k=1: 3$

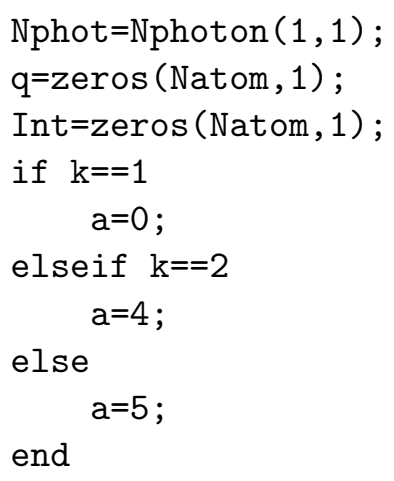


Simulation of the radial ionization dynamics:

$\% \% \%$ Dynamic simulation. Start with q_i $=0$.

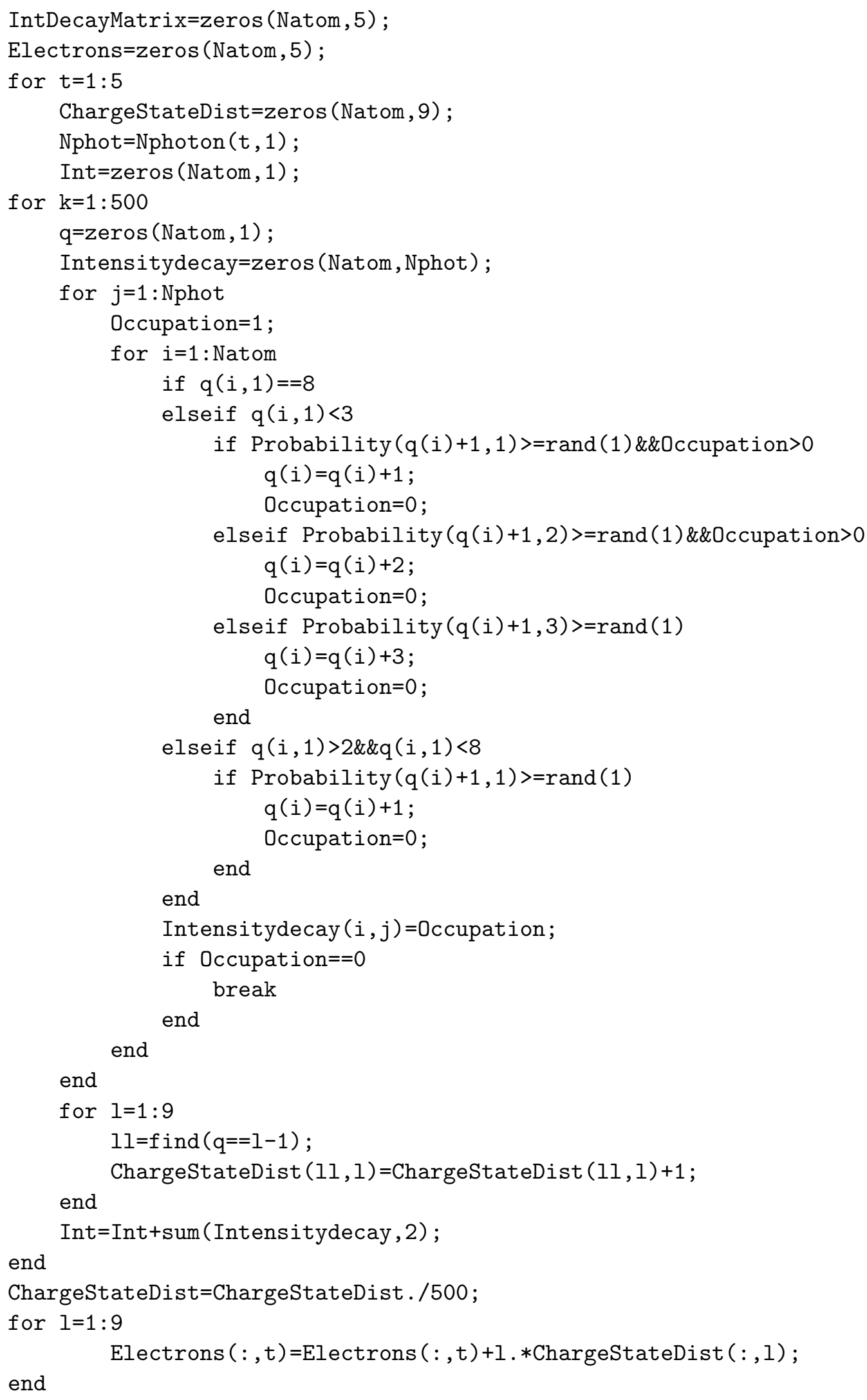




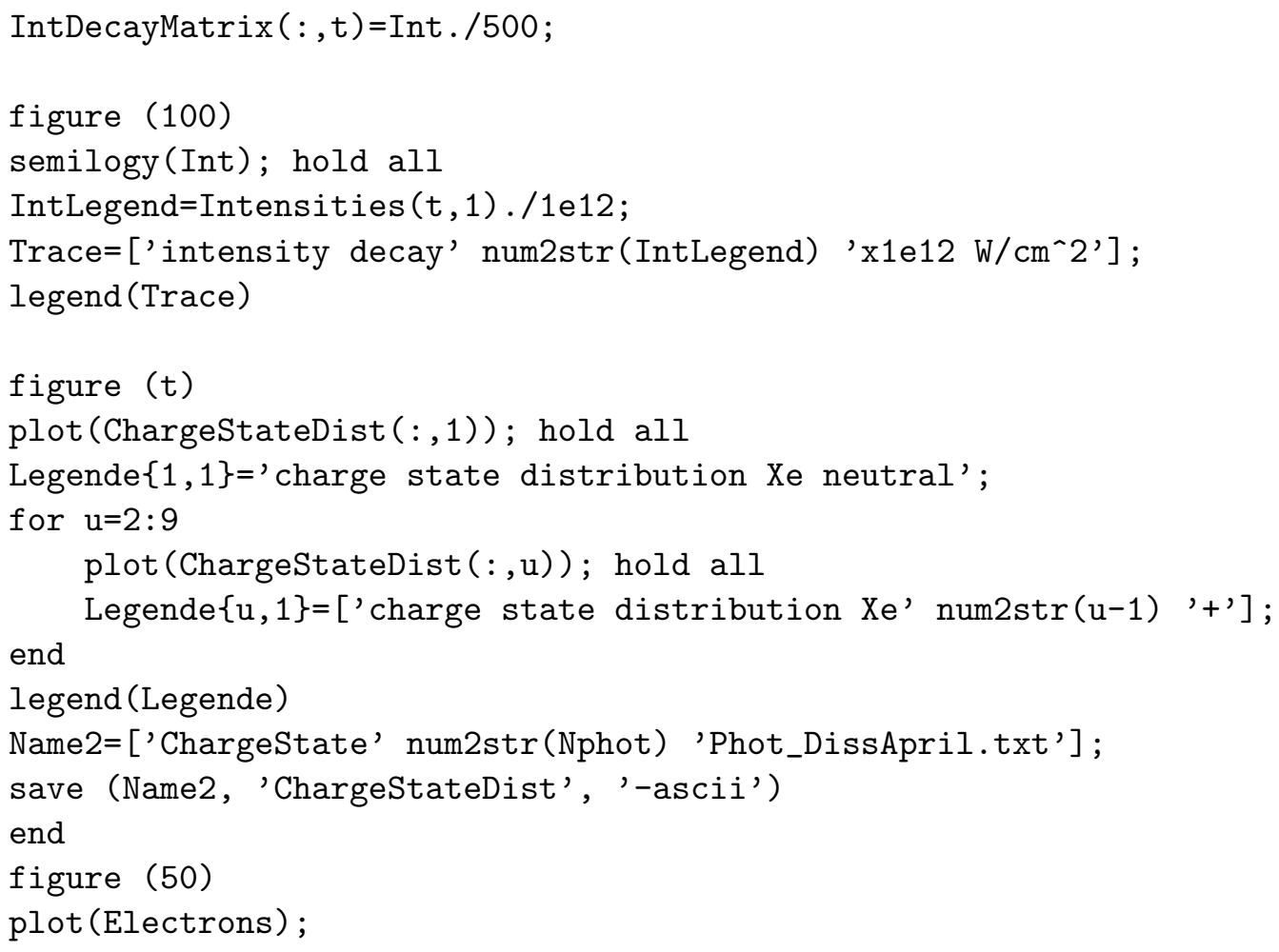

Factor $3 / 2$ in absorption probability: The probability $p_{i}$ of an atom $i$ in the simulation to absorb a photon is given by the ratio of tabulated absorption cross-sections $\sigma_{a b s}$ and geometric cross-section $\sigma_{g e o}$, times a factor of $3 / 2$. This factor of $3 / 2$ results from the onedimensional approximation, which can be demonstrated by calculating the probability for the single absorption process $p_{i}$ from the probability to find a photon at the penetration depth $l_{a b s}$ (also referred to as absorption length, hence the name) which is defined as $1 / e$.

$$
P\left(d=l_{a b s}\right)=e^{-1}=\left(1-p_{i}\right)^{N},
$$

with $N$ being the number of atoms from the surface to the penetration depth. $N$ can be calculated as $N=l a b s / 2 r_{a}$ where $r_{a}$ denotes the atomic radius of xenon. The connection between the penetration depth and the absorption cross-section can be found in the decay of the intensity

$$
I(d)=I_{0} \cdot e^{-n_{a} \sigma_{a b s} d},
$$

with the the incident intensity $I_{0}$ and the atomic density $n_{a}$. The intensity at the penetration depth has decayed to $1 / e$ of the incident intensity

$$
\begin{aligned}
I\left(l_{a b s}\right)=e^{-1} \cdot I_{0} & =I_{0} \cdot e^{-n_{a} \sigma_{a b s} l_{a b s}} \\
& \Leftrightarrow l_{a b s}=\frac{1}{n_{a} \sigma_{a b s}} .
\end{aligned}
$$

The atomic density $n_{a}$ can be expressed in terms of the atomic radius

$$
n_{a}=\frac{1}{V_{a}}=\left(\frac{4 \pi}{3} r_{a}^{3}\right)^{-1} .
$$

The geometric cross-section $\sigma_{g e o}=\pi r_{a}^{2}$ can be substituted into Eq. 4, yielding

$$
n_{a}=\frac{1}{\frac{4}{3} r_{a}^{3} \sigma_{g e o}} .
$$


The penetration depth in Eq. 3 can now be expressed through the cross-sections by inserting Eq. 5:

$$
l_{a b s}=\frac{4 r_{a} \sigma_{g e o}}{3 \sigma_{a b s}}
$$

This expression for $l_{a b s}$ can be substituted into the probability Eq. 1 and solved for $p_{i}$

$$
\begin{array}{r}
e^{-1}=\left(1-p_{i}\right)^{\frac{2}{3} \frac{\sigma g e o}{\sigma_{a b s}}} \\
p_{i}=1-e^{-\frac{3}{2} \frac{\sigma_{a b s}}{\sigma_{g e o}}}
\end{array}
$$

For absorption cross-sections $\sigma_{a b s} \ll \sigma_{g e o}$ the exponential function can be replaced by the first and second term of the Taylor series $e^{x} \approx 1+x$. Thus, the probability for absorption for a single atom $p_{i}$ reads

$$
p_{i}=\frac{3}{2} \frac{\sigma_{a b s}}{\sigma_{g e o}} .
$$




\section{List of Figures}

1.1 Imaging of single biomolecules . . . . . . . . . . . . . . . . . 2

2.1 Characteristic dipole emission of an oscillating point charge . . . . . . . . 10

2.2 Resonant behavior of bound electrons . . . . . . . . . . . . . . . . . . 12

2.3 Geometry and fields of the simple case of light scattering from a sphere. . . . 15

2.4 Coordinate systems for incident and scattered waves $\ldots \ldots \ldots$

2.5 Geometry and fields of a core-shell system. . . . . . . . . . . . . . . . . . 22

2.6 Radial and angular components of the normal modes. . . . . . . . . . . . . 24

2.7 Extinction paradox close to atomic resonances . . . . . . . . . . . . . 25

2.8 Calculated profiles of homogeneous and coated spheres . . . . . . . . . . 27

2.9 Photon energy dependent dominant process for the onset of ionization. . . . . 30

2.10 Photon energy and intensity dependent regimes . . . . . . . . . . . . . . . 31

2.11 Absorption cross-sections of atomic xenon and ions . . . . . . . . . . . 33

2.12 Ionization of xenon gas in intense $93 \mathrm{eV}$ pulses $\ldots \ldots \ldots$. . . . . . . . 35

2.13 Description of laser-cluster interaction in three phases . . . . . . . . . . 36

2.14 Many-body recombination . . . . . . . . . . . . . . . . . . 39

2.15 Key mechanisms of plasma heating in intense IR pulses . . . . . . . . . . . 40

2.16 Xenon clusters in intense VUV pulses . . . . . . . . . . . . . . . . . . 42

2.17 Cluster ionization at $38 \mathrm{eV}$ can be described by sequential multistep absorption. 43

2.18 Results from electron and ion spectra of small xenon clusters at $90 \mathrm{eV}$. . . 44

2.19 From hydrodynamic expansion to Coulomb explosion . . . . . . . . . . . 46

2.20 Scattering experiments on individual xenon clusters at $90 \mathrm{eV}$ photon energy. . 47

2.21 Suppressed recombination of clusters in soft X-ray pulses . . . . . . . . . 48

3.1 Peak Brilliance per year and light sources . . . . . . . . . . . . . . . 52 
3.2 Radiation of bending magnets, wigglers, undulators and FELs . . . . . . . . 53

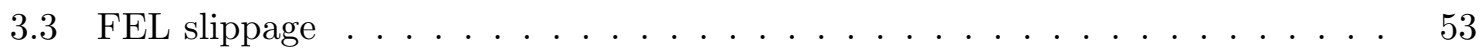

3.4 Low gain and high gain FELs . . . . . . . . . . . . . . . . . 54

3.5 Gain length and microbunching . . . . . . . . . . . . . 55

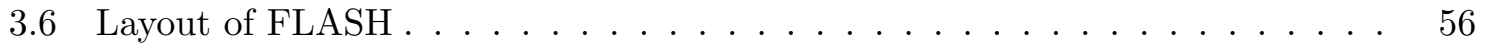

3.7 Gracing incidence mirrors $\ldots \ldots \ldots \ldots \ldots \ldots \ldots$

3.8 Multilayer mirrors for normal incidence reflection in XUV . . . . . . . . 58

3.9 Setup for coincident imaging and ion spectroscopy of single clusters . . . . . . 59

3.10 Supersonic expansion and cluster growth . . . . . . . . . . . . . 60

3.11 Scheme of adiabatic expansion and cluster condensation . . . . . . . . . 62

3.12 Transition from monomer addition to cluster aggregation $\ldots \ldots \ldots$. . . . . 63

3.13 Funktion principle of the solenoid valve and stable conditions in pulsed nozzle operation. . . . . . . . . . . . . . . . . 64

3.14 MCP phosphor stack with center hole and detector geometry . . . . . . . 65

3.15 Bipolar TOF and detector components . . . . . . . . . . . . . . 68

3.16 MCP efficiency with mass and impact energy. . . . . . . . . . . . . . 69

3.17 Scanning for highest focal power density . . . . . . . . . . . . . 70

4.1 Timescales addressed by simultaneous imaging and ion mass spectrometry . . 71

4.2 Influence of focal averaging and size distributions . . . . . . . . . . . 72

4.3 Statistics in single cluster mode . . . . . . . . . . . . . . . . . 73

4.4 Limit of single cluster mode: Newton rings _ . . . . . . . . . . . 75

4.5 Measured and simulated Newton rings with Moirée effect . . . . . . . . . 76

4.6 Selection of different cluster scattering patterns . . . . . . . . . . . . 78

4.7 Resolving surface structures at high q-values f . . . . . . . . . . . 79

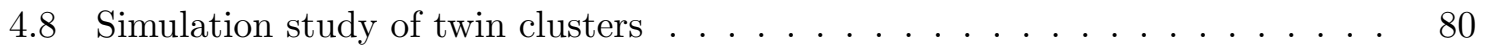

4.9 Comparison of experimental and simulated patterns of twin clusters _ . . . . 81

4.10 Simulating patterns of twin, triple and hailstone structures $\ldots \ldots \ldots$. . . 82

4.11 Scattering signal, cluster sizes and hit statistics versus source trigger delay . . 83

4.12 Cluster size distributions after the main cluster pulse . . . . . . . . . 85

4.13 Cluster source trigger delay scan for atomic signal $\ldots \ldots \ldots$

4.14 Enhanced cluster growth during the valve closing . . . . . . . . . . . 86 
4.15 Rayleigh scattering of a pulsed cluster beam . . . . . . . . . . . . 87

4.16 Scattering patterns and ion spectra of clusters with $R=400 \mathrm{~nm} \ldots \ldots$. . . 88

4.17 Simulated trajectories in the bipolar tof. . . . . . . . . . . . . . . 90

4.18 Time-to-energy conversion function of the bipolar tof. . . . . . . . . . . . 91

4.19 Transmission function of the bipolar tof $\ldots \ldots \ldots$. . . . . . . . 92

4.20 Ion spectrum of $400 \mathrm{~nm}$ cluster analyzed with SIMION simulation $\ldots \ldots . . \quad 93$

4.21 Size sorted ion spectra from 80 to $600 \mathrm{~nm}$ radius $\ldots \ldots \ldots$. . . . . . . 95

4.22 Assignment of epd values to the scans. . . . . . . . . . . . . . . . . 96

4.23 Linear behavior of the kinetic energy per charge state . . . . . . . . . 97

4.24 Compare calculated to experimentally obtained KEDI. . . . . . . . . . . . . . 99

4.25 Electrostatic models of a quasi-neutral nanoplasma . . . . . . . . . . . . . . 101

4.26 Total charge on the cluster from the electrostatic model of a charged surface. 102

4.27 Illustration of surface explosion and core recombination . . . . . . . . . 104

4.28 Size sorted epd scans for radii $180-600 \mathrm{~nm} \ldots \ldots \ldots$

4.29 Comparison of simulated ring, sphere, and smooth ring patterns. . . . . . . 107

4.30 Time-binning of the evolution of the nanoplasma $\ldots \ldots$. . . . . . . . 109

4.31 Time binning of averaged profiles from $R=400 \mathrm{~nm}$ clusters $\ldots \ldots \ldots$

4.32 Systematic simulations of coated spheres . . . . . . . . . . . . . . . 112

4.33 Simulated profiles matching the time-binned experimental data . . . . . . . 114

4.34 Asymmetric ionization dynamics . . . . . . . . . . . . . . . . . . . . 114

4.35 Values of the fit parameters for calculated profiles . . . . . . . . . . . 115

4.36 Atomic scattering factor $f_{1}$ of $\mathrm{Xe}^{3+}$ in the XUV regime . . . . . . 118

4.37 One-dimensional simulation of radial ionization dynamics $\ldots \ldots \ldots$. . . . . 119

4.38 Simulated radial development of the charge state densities . . . . . . . . . . 121

4.39 Simulated intensity decay over distance from the cluster surface . . . . . . . 122

4.40 Emergence of a transient plasma multilayer. . . . . . . . . . . . . . . . 124

5.1 Imaging a Mie plasmon $\ldots \ldots \ldots \ldots \ldots \ldots$

2 Efficiency calibration with IR-induced fluorescence light . . . . . . . . . . 129

3 Higher fluorescence yield in IR-pump-XUV-probe configuration . . . . . . . . 130

4 Areas with decreased efficiency and correction curve . . . . . . . . . . . . 130 
$5 \quad$ Tof light peak versus scattering detector signal . . . . . . . . . . . . . . 132 


\section{Bibliography}

[1] L. Pauling. The place of chemistry in the integration of sciences. Main currents in modern thought, 7(110), 1950.

[2] For his pioneering work in femtosecond spectroscopy, A. Zewail received the Nobel prize in physics in 1999.

[3] A.H. Zewail. Femtochemistry. Past, present, and future. Pure and Applied Chemistry, 72(12):2219-2231, 2000.

[4] P.B. Corkum and F. Krausz. Attosecond science. Nature Physics, pages 381-387, 2007.

[5] For the discovery of X rays, W. C. Röntgen was awarded the first Nobel prize in physics in 1901.

[6] Photoelectron spectroscopy was developed by K. Siegbahn who was awarded the Nobel prize in physics in 1981.

[7] M. Laue was awarded the Nobel prize in physics in 1914 for the discovery of X-ray diffraction from crystals.

[8] W. and L. Bragg received the Nobel prize in physics in 1915 for developing X-ray diffraction on crystals as a method for structure analysis.

[9] M. Perutz and J. Kendrew were awarded the Nobel prize in chemistry in 1962 for determining the structure of hemoglobin and myoglobin.

[10] The Nobel prize in chemistry in 1964 was given to D Crowfoot Hodgkin for determining the structure of penicillin.

[11] List of nobel prizes for structural determination of molecules. http://proteopedia.org/ wiki/index.php/Nobel_Prizes_for_3D_Molecular_Structure, 2013.

[12] G.J. Palenik, W.P. Jensen, and I.H. Suh. The history of molecular structure determination viewed through the Nobel prizes. Journal of Chemical Education, 80(7):753-761, 2003.

[13] L. Slabinski and L. Jaroszewski. The challenge of protein structure determination lessons from structural genomics. Protein Science, pages 2472-2482, 2007.

[14] J. Feldhaus, J. Arthur, and J. Hastings. X-ray free-electron lasers. Journal of Physics $B, 38: 799-819,2005$.

[15] J.R. Schneider. FLASH - from accelerator test facility to the first single-pass soft x-ray free-electron laser. Journal of Physics B, 43:194001, 2010. 
[16] P. Emma et al. First lasing and operation of an ångstrom-wavelength free-electron laser. Nature Photonics, 4(9):641-647, 2010.

[17] A. Doerr. Diffraction before destruction. Nature Methods, 8(283), 2011.

[18] R. Neutze et al. Potential for biomolecular imaging with femtosecond X-ray pulses. Nature, 406(6797):752-7, 2000.

[19] C. Bostedt et al. Experiments at FLASH. Nuclear Instruments and Methods in Physics Research Section A: Accelerators, Spectrometers, Detectors and Associated Equipment, 601(1-2):108-122, 2009.

[20] C. Bostedt et al. Ultra-fast and ultra-intense x-ray sciences: First results from the Linac Coherent Light Source free-electron laser. submitted to Journal of Physics B,, 2013.

[21] M. Bogan et al. Single particle X-ray diffractive imaging. Nano Letters, 8(1):310-6, 2008.

[22] C. Bostedt et al. Clusters in intense FLASH pulses: ultrafast ionization dynamics and electron emission studied with spectroscopic and scattering techniques. Journal of Physics B, 43(19):194011, 2010.

[23] H. Chapman et al. Femtosecond X-ray protein nanocrystallography. 470(7332):73-77, 2011.

[24] D. Rupp et al. Identification of twinned gas phase clusters by single shot scattering with intense soft x-ray pulses. New Journal of Physics, 14:055016, 2012.

[25] M. Seibert et al. Single mimivirus particles intercepted and imaged with an X-ray laser. Nature, 470(7332):78-81, 2011.

[26] M. Bergh et al. Feasibility of imaging living cells at subnanometer resolutions by ultrafast X-ray diffraction. Quarterly Reviews of Biophysics, 41(3-4):181-204, 2008.

[27] S. Son, L. Young, and R. Santra. Impact of hollow-atom formation on coherent x-ray scattering at high intensity. Physical Review A, 83(3):1-11, 2011.

[28] B. Ziaja et al. Limitations of coherent diffractive imaging of single objects due to their damage by intense x-ray radiation. New Journal of Physics, 14(11):115015, 2012.

[29] U. Saalmann, C. Siedschlag, and J.M. Rost. Mechanisms of cluster ionization in strong laser pulses. Journal of Physics B, 39(4):R39-R77, 2006.

[30] S. Schorb et al. Size-Dependent Ultrafast Ionization Dynamics of Nanoscale Samples in Intense Femtosecond X-Ray Free-Electron-Laser Pulses. Physical Review Letters, 108:233401, 2012.

[31] M. Hoener et al. Charge recombination in soft x-ray produced nanoplasmas. Journal of Physics B, 41:181001, 2008.

[32] C. Bostedt et al. Ultrafast x-ray scattering of xenon nanoparticles: Imaging transient states of matter. Physical Review Letters,, 108:093401, 2012.

[33] O.F. Hagena. Nucleation and growth of clusters in expanding nozzle flows. Surface Science, 106:101-116, 1981. 
[34] S. Hau-Riege. High-Intensity X-rays - Interaction with Matter. John Wiley, 2011.

[35] D. Attwood. Soft x-ray and extreme ultraviolet radiation. Cambridge University Press, 2007.

[36] C.F. Bohren and D. Huffman. Absorption and scattering of light by small particles. John Wiley, 1983.

[37] J.C. Maxwell. A dynamical theory of the electromagnetic field. Philosophical Transactions of the Royal Society of London, 1865.

[38] M. Born and E. Wolf. Principles of Optics, 7th edition. Cambridge University Press, 1999.

[39] K.E. Peiponen et al. Kramers-Kronig relations and sum rules of negative refractive index media. The European Physical Journal B, 41(1):61-65, 2004.

[40] J.J. Thomson. Conduction of electricity through gases. Cambridge University Press, 1906.

[41] J. Strutt. On the scattering of light by small particles. Philosophical Magazine, (4):447454 .

[42] E. Hecht. Optik, 4. Edition. Oldenbourg Verlag, 2005.

[43] G. Mie. Beiträge zur Optik trüber Medien, speziell kolloidaler Metallösungen. Annalen der Physik, 1908.

[44] C. Peltz et al. Fully microscopic analysis of laser-driven finite plasmas using the example of clusters. New Journal of Physics, 14(6):065011, 2012.

[45] M. Kerker. The scattering of light and other electromagnetic radiation. Academic Press New York, 1969.

[46] L. Liu et al. Improved algorithm of light scattering by a coated sphere. China Particuology, 5:230-236, 2007.

[47] A.L. Aden and M. Kerker. Scattering of Electromagnetic Waves from Two Concentric Spheres. Journal of Applied Physics, 22(10):1242, 1951.

[48] R.A. Meyer and A. Brunsting. Light Scattering from Nucleated Biological Cells. Biophysical Journal, 15:191-203, 1975.

[49] www.scattport.org/files/jianqi_shen/. Web link for the code package by Jianqi Shen for coated spheres, 2012.

[50] L. Novotny and B. Hecht. Principles of Nano-Optics. Cambridge University Press, 2008.

[51] A.A. Sorokin et al. Photoelectric effect at ultrahigh intensities. Physical Review Letters, 99(21):213002, 2007.

[52] C. Cohen-Tannoudji, B. Diu, and F. Laloe. Quantenmechanik 2. deGruyter Verlag, 1999.

[53] M. Richter et al. Multiphoton ionization of atoms with soft x-ray pulses. Journal of Physics B, 43(19):194005, 2010. 
[54] V. Richardson et al. Two-Photon Inner-Shell Ionization in the Extreme Ultraviolet. Physical Review Letters, 105(1):013001, 2010.

[55] L. Young et al. Femtosecond electronic response of atoms to ultra-intense X-rays. Nature, 466(7302):56-61, 2010.

[56] N. Berrah et al. Non-linear processes in the interaction of atoms and molecules with intense EUV and X-ray fields from SASE free electron lasers (FELs). Journal of Modern Optics, 57(12):37-41, 2010.

[57] G. Doumy et al. Nonlinear atomic response to intense ultrashort x rays. Physical Review Letters, 106(8):083002, 2011.

[58] B. Rudek et al. Ultra-efficient ionization of heavy atoms by intense X-ray free-electron laser pulses. Nature Photonics, 2012.

[59] T. Fennel et al. Laser-driven nonlinear cluster dynamics. Reviews of Modern Physics, 82(2):1793-1842, 2010.

[60] http://henke.lbl.gov/optical_constants/. Online data base for X-ray optical constants.

[61] J.B. West. Photoionization of atomic ions. Journal of Physics B, 45, 2001.

[62] D.L. Ederer and M. Manalis. Photoabsorption of the 4d electrons in xenon. J. Opt. Soc. Am., 65(6):634-637, 1975.

[63] Y. Itoh, A. Ito, and M. Kitajima. Absolute photoionization cross section measurements of $\mathrm{Xe}^{+}$ions in the $4 \mathrm{~d}$ threshold energy region. Journal of Physics B, 4075(01):3493$3499,2001$.

[64] P. Andersen et al. Absolute cross sections for the photoionization of $4 \mathrm{~d}$ electrons in $\mathrm{Xe}^{+}$and $\mathrm{Xe}^{2+}$ ions. Journal of Physics B, 4075(01):2009-2019, 2009.

[65] E.D. Emmons et al. Photoionization and electron-impact ionization of $\mathrm{Xe}^{3+}$. Physical Review A, 71(042704), 2005.

[66] A. Aguilar et al. Absolute photoionization cross sections for $\mathrm{Xe}^{4+}, \mathrm{Xe}^{5+}$, and $\mathrm{Xe}^{6+}$ near $13.5 \mathrm{~nm}$ : Experiment and theory. Physical Review A, 73(3):1-10, 2006.

[67] J. Bizau et al. Photoionization of highly charged ions using an ECR ion source and undulator radiation. Physical Review Letters, 84(3):435-8, 2000.

[68] J.W. Cooper. Interaction of maxima in the absorption of soft X rays. Physical Review Letters, 1(25):4-6, 1964.

[69] M.G. Mayer. Rare-earth and transuranic elements. Physical Review, pages 1-4, 1941.

[70] K.T. Cheng and W.R. Johnson. Orbital collapse and the photoionization of the inner 4d shells for Xe-like ions. Physical Review A, 28(5), 1983.

[71] T. Luhmann et al. Final ion-charge resolving electron spectroscopy for the investigation of atomic photoionization processes: Xe in the region of the $4 d-\epsilon f$ resonance. Physical Review A, 57(1):282-291, 1998.

[72] H. Haberland in Bergmann Schäfer. Lehrbuch der Experimentalphysik 5. Gase, Nanosysteme, Flüssigkeiten. deGruyter Verlag, 2009. 
[73] J. Posthumus. Molecules and Clusters in Intense Laser Fields. Cambridge University Press, 2009.

[74] V.P. Krainov and M.B. Smirnov. Cluster beams in the super-intense femtosecond laser pulse. Physics Reports, 370(3):237-331, 2002.

[75] U. Saalmann. Cluster nanoplasmas in strong FLASH pulses: formation, excitation and relaxation. Journal of Physics B, 43(19):194012, 2010.

[76] P.G. Reinhard and E. Suraud. Introduction to Cluster Dynamics. Wiley, 2004.

[77] H. Wabnitz et al. Multiple ionization of atom clusters by intense soft X-rays from a free-electron laser. Nature, 420(6915):482-5, 2002.

[78] I. Last and J. Jortner. Quasiresonance ionization of large multicharged clusters in a strong laser field. Physical Review A, 60(3):2215-2221, 1999.

[79] M. Arbeiter and T. Fennel. Rare-gas clusters in intense VUV, XUV and soft xray pulses: signatures of the transition from nanoplasma-driven cluster expansion to Coulomb explosion in ion and electron spectra. New Journal of Physics, 13(5):053022, 2011.

[80] C. Bostedt et al. Fast electrons from multi-electron dynamics in xenon clusters induced by inner-shell ionization. New Journal of Physics, 12(8):083004, 2010.

[81] C. Jungreuthmayer et al. Intense VUV laser cluster interaction in the strong coupling regime. Journal of Physics B, 38(16):3029-3036, 2005.

[82] T. Fennel, L. Ramunno, and T. Brabec. Highly Charged Ions from Laser-Cluster Interactions: Local-Field-Enhanced Impact Ionization and Frustrated Electron-Ion Recombination. Physical Review Letters, 99(23):1-4, 2007.

[83] A. Gets and V. Krainov. The ionization potentials of atomic ions in laser-irradiated Ar, Kr and Xe clusters. Journal of Physics B, 39(7):1787-1795, 2006.

[84] C. Deiss et al. Laser-Cluster Interaction: X-Ray Production by Short Laser Pulses. Physical Review Letters, 96:013202, 2006.

[85] J. Passig et al. Nanoplasmonic electron acceleration in silver clusters studied by angularresolved electron spectroscopy. New Journal of Physics, 14(8):085020, 2012.

[86] L. Köller et al. Plasmon-Enhanced Multi-Ionization of Small Metal Clusters in Strong Femtosecond Laser Fields. Physical Review Letters, 82(19):3783-3786, 1999.

[87] D. Semkat, R. Redmer, and T. Bornath. Collisional absorption in aluminum. Physical Review E, 73(6):066406, 2006.

[88] T. Ditmire et al. Explosion of atomic clusters heated by high-intensity femtosecond laser pulses. Physical Review A, 57(1):369-382, 1998.

[89] H. Milchberg, S. McNaught, and E. Parra. Plasma hydrodynamics of the intense lasercluster interaction. Physical Review E, 64(5):056402, 2001.

[90] M. Lezius et al. Explosion Dynamics of Rare Gas Clusters in Strong Laser Fields. Physical Review Letters, 62(2):261-265, 2000. 
[91] T. Ditmire et al. Interaction of intense laser pulses with atomic clusters. Physical Review A, 53(5):3379-3402, 1996.

[92] T. Ditmire et al. High Intensity Laser Absorption by Gases of Atomic Clusters. Physical Review Letters, 78(16):3121-3124, 1997.

[93] S. Sakabe et al. Skinning of argon clusters by Coulomb explosion induced with an intense femtosecond laser pulse. Physical Review A, 74(4):043205, 2006.

[94] H. Thomas et al. Shell explosion and core expansion of xenon clusters irradiated with femtosecond soft x-ray pulses. Journal of Physics B, 42:134018, 2009.

[95] K. Ishikawa and T. Blenski. Explosion dynamics of rare-gas clusters in intense laser field. Physical Review A, 62:063204, 2000.

[96] M.R. Islam, U. Saalmann, and J.M. Rost. Kinetic energy of ions after Coulomb explosion of clusters induced by an intense laser pulse. Physical Review A, 73:041201, 2006 .

[97] T. Laarmann et al. Emission of Thermally Activated Electrons from Rare Gas Clusters Irradiated with Intense VUV Light Pulses from a Free Electron Laser. Physical Review Letters, 95:063402, 2005.

[98] R. Santra and C. Greene. Xenon Clusters in Intense VUV Laser Fields. Physical Review Letters, 91(23):1-4, 2003.

[99] D. Bauer. Small rare gas clusters in laser fields: ionization and absorption at long and short laser wavelengths. Journal of Physics B, 37(15):3085-3101, 2004.

[100] C. Siedschlag and J.M. Rost. Small Rare-Gas Clusters in Soft X-Ray Pulses. Physical Review Letters, 93(4):2-5, 2004.

[101] B. Ziaja et al. Energetics, Ionization, and Expansion Dynamics of Atomic Clusters Irradiated with Short Intense Vacuum-Ultraviolet Pulses. Physical Review Letters, 102(20):205002, 2009.

[102] C. Bostedt et al. Multistep ionization of argon clusters in intense femtosecond extreme ultraviolet pulses. Physical Review Letters, 100(13):133401, 2008.

[103] M. Arbeiter and T. Fennel. Ionization heating in rare-gas clusters under intense XUV laser pulses. Physical Review A, 82(1):1-7, 2010.

[104] T. Gorkhover et al. Nanoplasma dynamics of single large xenon clusters irradiated with superintense x-ray pulses from the Linac Coherent Light Source Free-Electron Laser. Physical Review Letters, 108:245005, 2012.

[105] S. Schorb. Size-dependent ultrafast ionization dynamics of nanoscale samples in intense femtosecond x-ray free-electron laser pulses. PhD thesis, TU Berlin, Oct 2012.

[106] Z Huang and KJ Kim. Review of x-ray free-electron laser theory. Physical Review Special Topics, 10:034801, 2007.

[107] W.C. Röntgen. On a new kind of rays. Nature, 420(53):274-276, 1896.

[108] W. Ackermann et al. Operation of a free-electron laser from the extreme ultraviolet to the water window. Nature Photonics, 1(6):336-342, 2007. 
[109] J. Schneider et al. FLASH. The Free-Electron Laser in Hamburg. Deutsches Elektronen Synchrotron DESY, 2007.

[110] J. Falta and T. Möller. Forschung mit Synchrotronstrahlung. Vieweg und Teubner, 2010.

[111] I. Flegel et al. BlitzLicht. Deutsches Elektronen Synchrotron DESY, 2007.

[112] P. Schmüser. Fel theory for pedestrians. Lecture at Heraeus seminar Free-electron lasers: From fundamentals to applications, May 2012.

[113] R. Mitzner et al. Spatio-temporal coherence of free-electron laser pulses in the soft x-ray regime. Optics Express, 2008.

[114] E. Allaria et al. The FERMI@Elettra free-electron laser source for coherent x-ray physics: photon properties, beam transport system and applications. New Journal of Physics, 12:075002, 2010.

[115] J. Bödewadt. sFLASH - first results of direct seeding at FLASH. Proceedings of FEL 2010, Malmö, Sweden.

[116] J. Feldhaus et al. Possible application of X-ray optical elements for reducing the spectral bandwidth of an X-ray SASE FEL. Optics Communications, 140:341-352, 1997.

[117] E. Saldin et al. X-ray FEL with a meV bandwidth. Nuclear Instruments and Methods in Physics Research A, 475:357-362, 2001.

[118] J. Amann et al. Demonstration of self-seeding in a hard-X-ray free-electron laser. Nature Photonics, 180, 2012.

[119] http://flash.desy.de. DESY homepage.

[120] J. Rossbach. Short-wavelenght single-pass free-electron lasers. Proceedings of LINAC2002, 2002.

[121] K. Tiedtke et al. The soft x-ray free-electron laser FLASH at DESY: beamlines, diagnostics and endstations. New Journal of Physics, 11:023029, 2009.

[122] J. Feldhaus. FLASH - the first soft x-ray free-electron laser user facility. Journal of Physics B, 43:194002, 2010.

[123] B. Steeg et al. Total reflection amorphous carbon mirrors for vacuum ultraviolet freeelectron lasers. Applied Physic Letters, 84(5):657-659, 2004.

[124] T. Feigl et al. EUV multilayer optics. Microelectronic Engeneering, 83:703-706, 2006.

[125] M. Krikunova et al. Ionization dynamics in expanding clusters studied by XUV-pumpprobe spectroscopy. Journal of Physics B, 45:105101, 2012.

[126] M. Mueller et al. Fluorescence of xenon clusters as a probe for strong absorption XUV FEL pulses. in preparation, 2013.

[127] M. Sauppe. Untersuchung der Ionisationsdynamik von Xenonclustern mit zeitaufgelöster Massenspektroskopie und mit Streumethoden . Master Thesis, TU Berlin, May 2013. 
[128] O.F. Hagena. Cluster ion sources. Review of Scientific Instruments, 63(4):2374-2379, 1992.

[129] G. Scoles. Atomic and molecular beam methods 1. Oxford University Press, 1988.

[130] I. Yamada et al. Materials Processing by gas cluster ion beams. Materials Science and Engeneering, 34:231-295, 2001.

[131] http://encyclopedia.airliquide.com/encyclopedia.asp. Air Liquide online data base.

[132] J.M. Soler et al. Microcluster growth: Transition from successive monomer addition to coagulation. Physical Review Letters, 49(25):1856-1860, 1982.

[133] U. Buck and R. Krohne. Cluster size determination from diffractive He atom scattering. The Journal of Chemical Physics, 105(13):5408, 1996.

[134] F. Dorchies et al. Spatial distribution of cluster size and density in supersonic jets as targets for intense laser pulses. Physical Review A, 68:023201, 2003.

[135] http://www.parker.com/Literature/LiteratureFiles/PrecisionFluidicsDivision/ UpdatedFiles/PulseValves.pdf. Data sheet Paker - General Valve, pulsed solenoid UHV valve 99 series.

[136] G. Chen et al. Pressure dependence of argon cluster size for different nozzle geometries. Journal of Applied Physics, 106:053507, 2009.

[137] H. Thomas. Wechselwirkung von Edelgas-Clustern mit intensiven Pulsen weicher Röntgenstrahlung vom Freie-Elektronen-Laser FLASH. PhD Thesis, TU Berlin, 2009.

[138] Photonis. Quote and technical specifications of apd 3075 ps 32/28/8 i edr60:1 csi 3.0ch p20, 2011.

[139] J.L. Wiza et al. Microchannel Plate Detectors. Nuclear Instruments and Methods, 162:587-601, 1979.

[140] W.B. Colson et al. High-gain imaging electron multiplier. Review of Scientific Instruments, 44(12):1694-1696, 1973.

[141] I.S. Gilmore and M.P. Seah. Ion detection efficiency in SIMS: dependencies on energy, mass and composition for microchannel plates used in mass spectrometry. International Journal of Mass Spectrometry, 202:217-229, 2000.

[142] http://www.home.agilent.com/en/pd-1197891-pn-U1056B. Acquiris data sheet.

[143] W.C. Wiley and I.H. McLaren. Time-of-Flight Mass Spectrometer with Improved Resolution. Rev. Sci. Instrum., 12(26):1150, 1955.

[144] M. Adolph. Observation of cluster growing processes using imaging techniques. PHD Thesis, TU Berlin, September 2013.

[145] http://HDGroup.com. HD group homepage.

[146] A.R.B. de Castro et al. Numerical simulation of small angle scattering (SAXS) for large atomic clusters. Journal of Electron Spectroscopy and Related Phenomena, 166-167:21$27,2008$. 
[147] S. Roling et al. Temporal and spatial coherence properties of free-electron-laser pulses in the extreme ultraviolet regime. Physical Review Special Topics - Accelerators and Beams, 406(080701), 2002.

[148] J. Farges et al. Structure and temperature of rare gas clusters in a supersonic expansion. Surface Science, 106:95-100, 1981.

[149] B. van de Waal. Cross-twinning model of fcc crystal growth. Journal of Crystal Growth, 158(1-2):153-165, January 1996.

[150] H. Chapman, A. Barty, and S. Marchesini. High-resolution ab initio three-dimensional x-ray diffraction microscopy. JOSA A, 2006.

[151] N. Meidinger et al. Next generation of pnCCDs for X-ray spectroscopy and imaging. Nuclear Instruments and Methods in Physics Research Section A: Accelerators, Spectrometers, Detectors and Associated Equipment, 568(1):141-148, 2006.

[152] D. Rupp et al. Publication in preparation.

[153] L. Shao-Hui et al. Investigation of the time characteristics of a pulsed flow of rare gas clusters. Chinese Physics, 12(8):856-860, 2003.

[154] S. Khmel and R. Sharafutdinov. Time-of-flight measurements in a molecular beam generated from a jet of condensing carbon dioxide. Journal of Technical Physics, 43(8):986989, 1998.

[155] www.SIMION.com. SIMION homepage, Feb 2013.

[156] D.A. Dahl, J.E. Delmore, and A.D. Appelhans. SIMION PC/PS2 electrostatic lense design program. Review of Scientific Instruments, 61(607), 1990.

[157] W. Demtröder. Experimentalphysik II, Elektrizität und Optik. Springer Verlag, 2009.

[158] T. Flueckiger et al. Publication in preperation.

[159] J. Nilsen, W.R. Johnson, and K.T. Cheng. Searching for plasmas with anomalous dispersion in the soft X-ray regime. Proc of SPIE, (6702), 2007.

[160] O. Björneholm et al. Core level photelectron and X-ray absorption spectroscopy of free argon clusters: Size-dependent energy shifts and determination of surface atom coordination. Physical Review Letters, 74(15):3017-3020, 1995.

[161] S. Micheau et al. Screening models for laser-cluster interactions. Journal of Physics B, 38(18):3405-3422, 2005.

[162] K. Binder. Monte Carlo methods in statistical physics. Springer Verlag, 1979.

[163] D.R. Sears and H.P. Klug. Density and Expansivity of Solid Xenon. Journal of Chemical Physics, 37(12):3002, 1962. 


\section{Eidesstattliche Versicherung}

Ich erkläre hiermit an Eides statt, dass ich die Dissertation mit dem Titel

Ionization and plasma dynamics of single large xenon clusters in superintense XUV pulses

selbstständig verfasst habe. Ich habe alle benutzten Hilfsmittel und Quellen aufgeführt und die Zusammenarbeit mit anderen Wissenschaftlern kenntlich gemacht.

Vorname / Name / Datum / Unterschrift 


\section{Acknowledgements}

Zum Gelingen dieser Arbeit haben eine Reihe von Menschen beigetragen, denen ich hiermit meinen Dank aussprechen möchte. An erster Stelle möchte ich Thomas Möller dafür danken, dass er mir die Möglichkeit gegeben hat in seiner Gruppe zu forschen und diese Arbeit anzufertigen. Er hat mich in allen Belangen unterstützt und mir Anleitung und Freiraum geboten. Ganz besonders bedanken möchte ich mich auch für all die Unterstützung bei Christoph Bostedt. Er hat mich bereits vor sechs Jahren für die Diplomarbeit zur FEL-crew geholt und hat mir zu zwei großartigen Aufenthalten an der LCLS verholfen. Stets war er mir ein sehr guter Freund und eine Quelle der Inspiration.

Ein großer Dank gebührt meinen Kolleginnen Leonie und Maria für ihre große Hilfe in stressigen Zeiten und der gesamten AG Möller für die angenehme und konstruktive Arbeitsatmosphäre im Büro, im Labor und auf Messzeiten. Danken möchte ich auch meinen Mitstreitern der ersten Stunde Marcus, Dave, Sebastian und Tais für die tolle Zusammenarbeit und die gute Zeit. Darüber hinaus möchte ich insbesondere Sebastian für den Extrasupport in den letzten Zügen dieser Arbeit danken. Ein weiteres großes Dankeschön möchte ich Felix Schnellbacher, Lila und Feli dafür aussprechen, dass sie geholfen haben, meine Texte verständlicher zu machen und mein oftmals schräges Englisch zu reparieren. Ebenso möchte ich Karl-Heinz Meiwes-Broer danken, der sich bereit erklärt hat, die Rolle des Zweitgutachters dieser Arbeit zu übernehmen.

Ein besonders herzliches Dankeschön geht an Jörn Six und das ganze Team der Feinmechanischen Werkstatt des IOAP, sowie an Sven Urban, ohne die gar nichts gegangen wäre. Sie haben die Ideen in meinem Kopf in echte Dinge verwandelt, die man waschen und ins Vakuum bauen kann, was mir immer noch unglaublich erscheint. Marion Magalowski und ihren Kollegen am Institut und der Uni im administrativen Bereich möchte ich danken, die mir aus einigem selbst verursachten Schlamassel geholfen haben.

Weiterhin möchte ich Thomas Fennel und seinen Doktoranden Christian Peltz und Matthias Arbeiter für lange, aufschlussreiche und für diese Arbeit äußerst wichtige Diskussionen danken. Ein großer Dank geht auch an Rolf Treusch, Sven Toleikis, Svea Kapitzki, Daniel Rolles und seine Gruppe, Sven Laarmann und seine Gruppe, sowie die Mitarbeiter der Desy-Werkstätten für ihre großartige Unterstützung und unzählige Rettungen in letzter Sekunde während der neun FLASH-Messzeiten, die ich bisher miterleben durfte.

Meine Freunde haben mich durch die letzten Monate ge- und ertragen, auch dafür möchte ich danke sagen. Ganz besonders bin ich Sandy und Dave, Annika, Caro und Juli für tausend Stunden babysitten und für-mich-da-sein dankbar. Auch meine Familien weit weg von Berlin, insbesondere Fritzi, Raimund, Brigitta und Rupert haben mich großartig unterstützt, danke dafür.

Meinem lieben Freund und Mann Tobi danke ich für sein unglaubliches Interesse an meiner Arbeit, einen freien Rücken, Geduld und Verständniss, den Antrieb zu dem er mir verholfen hat und für sein endloses Vertrauen in mich. Schließlich und endlich, danke an Jonna, du bist die Größte!

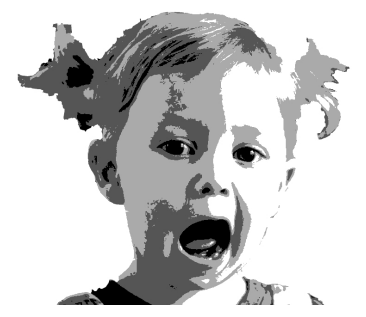

